\title{
Investigation of a Neural Network Approach in Modeling and Diagnostics of an Engine-out NOx Sensor
}

\author{
Zackery S. Layhew
}

Follow this and additional works at: https://researchrepository.wvu.edu/etd

\section{Recommended Citation}

Layhew, Zackery S., "Investigation of a Neural Network Approach in Modeling and Diagnostics of an Engine-out NOx Sensor" (2017). Graduate Theses, Dissertations, and Problem Reports. 6046.

https://researchrepository.wvu.edu/etd/6046

This Thesis is protected by copyright and/or related rights. It has been brought to you by the The Research Repository @ WVU with permission from the rights-holder(s). You are free to use this Thesis in any way that is permitted by the copyright and related rights legislation that applies to your use. For other uses you must obtain permission from the rights-holder(s) directly, unless additional rights are indicated by a Creative Commons license in the record and/ or on the work itself. This Thesis has been accepted for inclusion in WVU Graduate Theses, Dissertations, and Problem Reports collection by an authorized administrator of The Research Repository @ WVU. For more information, please contact researchrepository@mail.wvu.edu. 


\title{
Investigation of a Neural Network Approach in Modeling and Diagnostics of an Engine-out NOx Sensor
}

\author{
Zackery S. Layhew \\ Thesis submitted \\ to the Benjamin M. Statler College of \\ Engineering and Mineral Resources \\ at West Virginia University \\ in partial fulfillment of the requirements for the degree of \\ Master of Science in \\ Mechanical Engineering
}

Arvind Thiruvengadam Padmavathy, PhD, Committee Chairperson

Scott Wayne, PhD

Greg Thompson, $\mathrm{PhD}$

Department of Mechanical and Aerospace Engineering

Morgantown, West Virginia

2017

Keywords: Artificial Neural Network, NOx Sensor, NOx Prediction, OBD Copyright 2017 Layhew 


\section{ABSTRACT \\ Investigation of a Neural Network Approach in Modeling and Diagnostics of an Engine-out NOx Sensor}

\section{Zackery Layhew}

The objective of this study was to develop a method for fault detection of an engine-out oxides of nitrogen (NOx) sensor. The aim of the developed method was not only to isolate a fault with the NOx sensor, but to also diagnose faults in other engine subsystems that may result in higher engine-out NOx production. The developed fault diagnostics are aimed at providing reliable, accurate determinations of sensor output, in-lieu of physical sensors.

The data for the development of numerical models in this study was derived from in-use emissions data of a 2014 Freightliner equipped with a 2013 Cummins ISX15 engine. Data included engine control unit (ECU) data from a variety of vehicle operation in southern California that included interstate, highway, regional, local, and near dock locations.

For this method of fault detection, a virtual sensor was created using an artificial neural network (ANN) with an input configuration using 12 engine parameters, which provided the most accurate results in this study. These parameters included engine speed, engine torque, fuel rate, intake temperature, boost pressure, exhaust temperature, coolant temperature, oil pressure, the first derivative of engine speed, the first derivative of engine torque, the second derivative of engine speed, and the second derivative of engine torque. The neural network could then be used to predict expected NOx values. 
The ANN NOx model was trained on a subset of the data and later validated with another subset of the available ECU data. Two different sets of training data, and seven validation data sets were used for prediction evaluation. The study also included the insertion of fault data and run against the model to test for fault detection with the best performing data set. It was found that the network is able to predict NOx within 1-5\% at highway operation, when trained with highway data, enabling the detection of NOx sensor faults as well as faults in engine subsystems that were included in the input parameters for the neural network. Three different types of sensor failures, including a step, ramp, and square function failure, were implemented in the validation data, which caused an increase in error between the actual and predicted NOx production to increase between $15-200 \%$, creating the means of detection. 


\section{ACKNOWLEDGMENTS}

I would first like to thank my Committee Chairperson Dr. Arvind Thiruvengadam for providing me with the opportunity to do research in the field of engines and OBD. I would also like to thank my other committee members, Dr. Greg Thompson and Dr. Scott Wayne. A special thank you to Dr. Wayne for everything you have taught me, and the mentorship you have provided throughout my entire undergraduate career, from SAE to Formula to everything else in between.

Next I would like to thank Dr. Melissa Morris, Saroj Pradhan and Pragalath Thiruvengadam for all the help and support in completing my research.

To Pat and Pam, I cannot thank you enough for the support and family-like treatment you have provided in the last two years.

I would like to thank my parents for helping me achieve everything I have so far in my life and shaping me into the person I have become. Without them, I would never have been able to achieve the things I have so far in my life.

Finally, to my wife Emily who has dealt with me spending long days and nights at engineering, and always being as supportive and as helpful as possible. I am thankful to have a true partner. 


\section{Table of Contents}

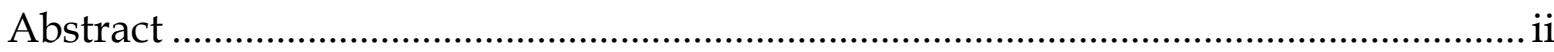

Acknowledgments ................................................................................................. iv

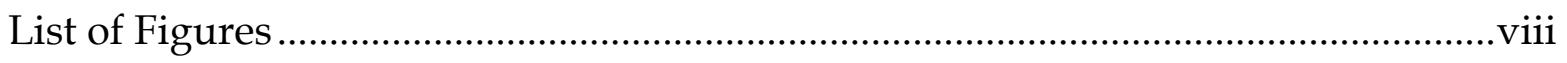

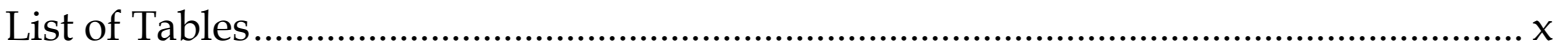

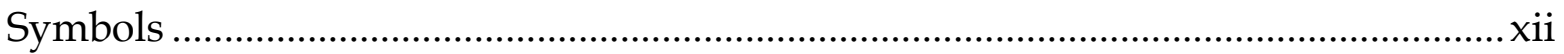

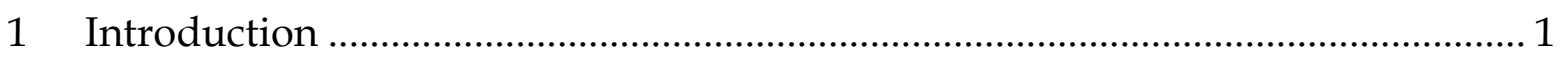

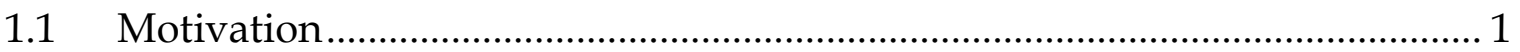

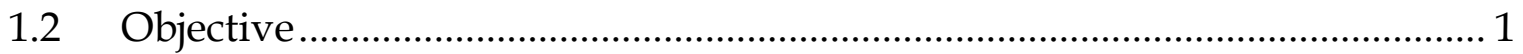

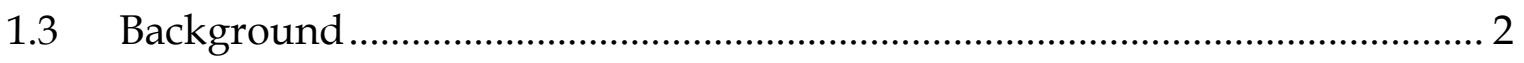

1.3.1 Diesel Engines and Emissions ......................................................... 2

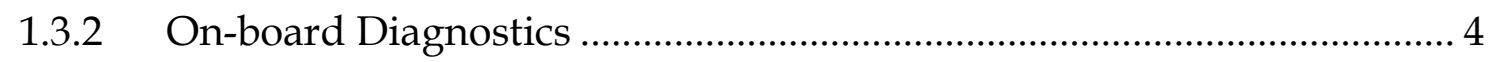

1.3.3 Artificial Neural Network ............................................................... 5

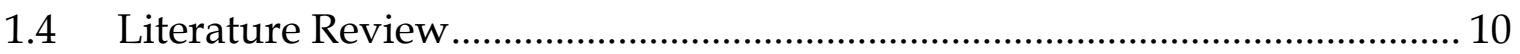

2 Experimental Data and Procedures ............................................................... 14

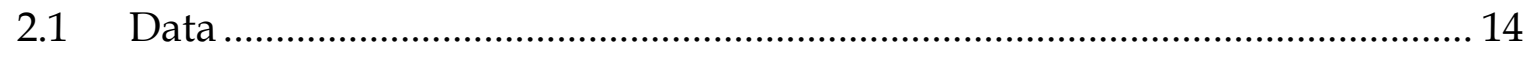

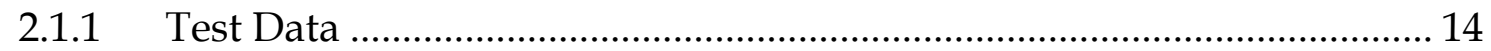

2.1.2 Interstate vs. Highway Data Comparison............................................. 16

2.1.3 Combined Training Data Set................................................................ 17

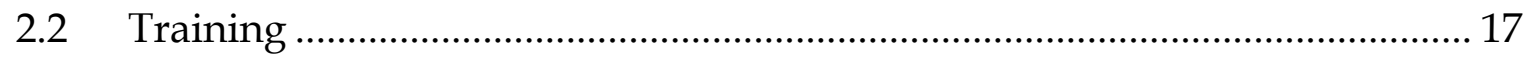

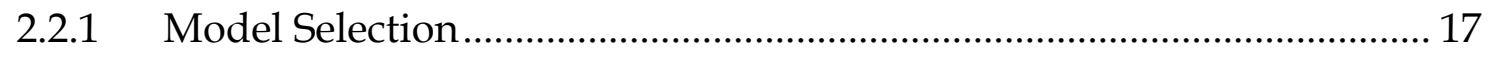

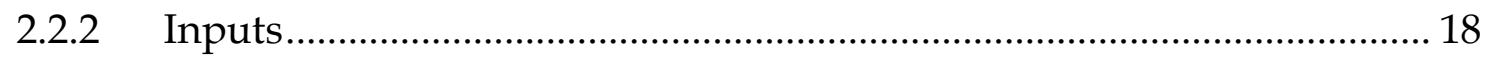

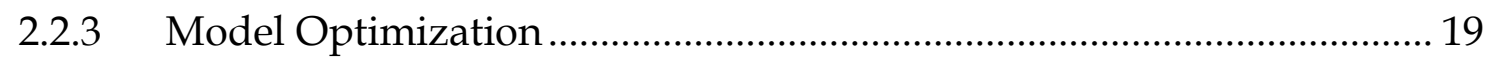

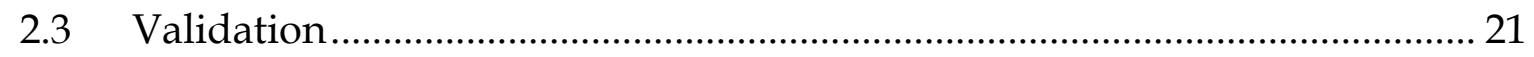

2.4 Exploration of Poor Prediction Areas ........................................................ 21

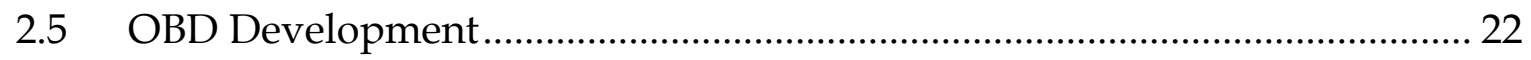

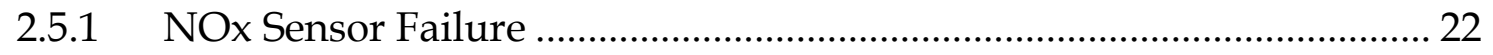

2.5.2 Boost Pressure Sensor Failure .............................................................. 23 


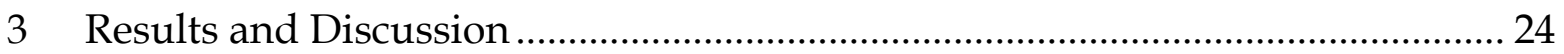

3.1 Neural Network Development .................................................................... 24

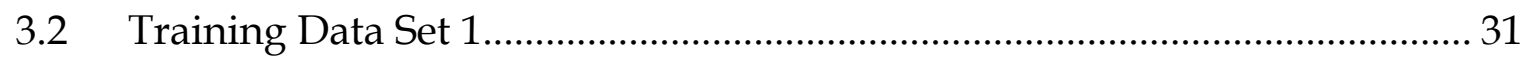

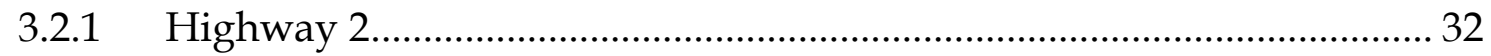

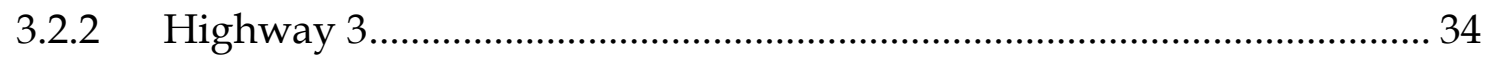

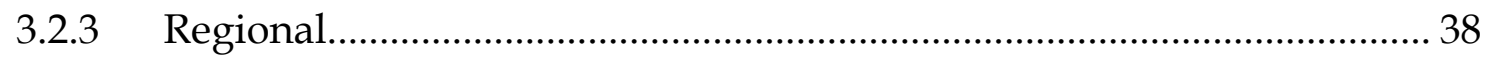

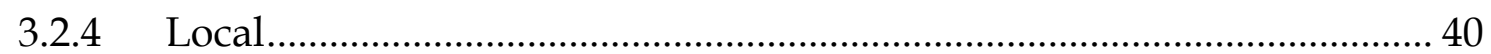

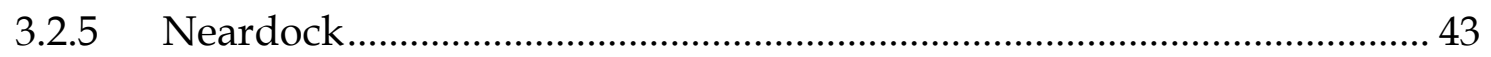

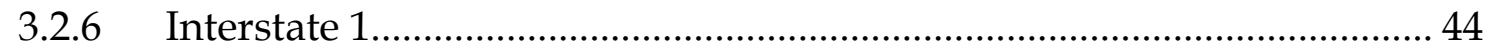

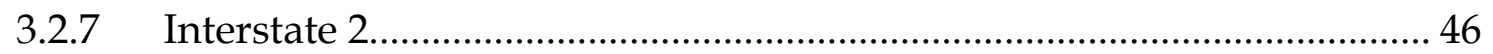

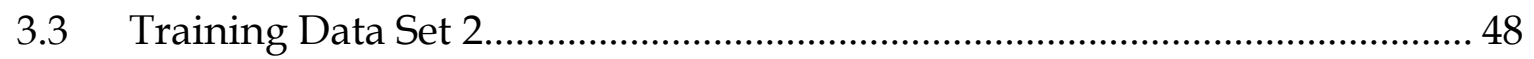

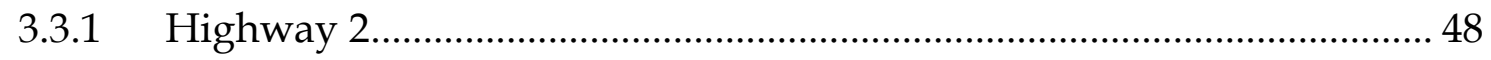

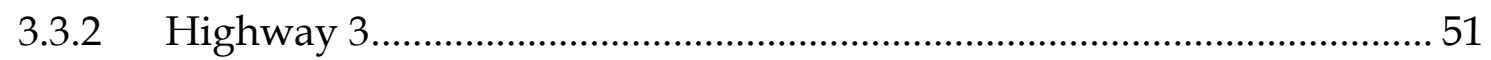

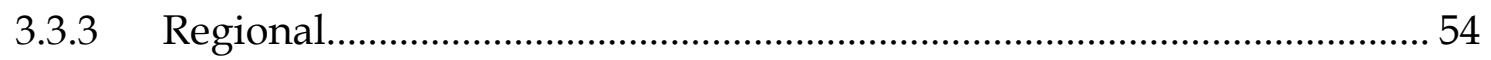

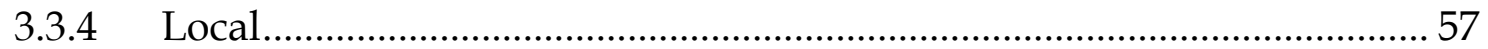

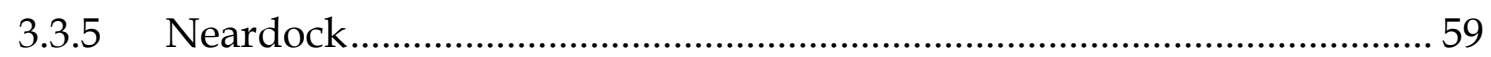

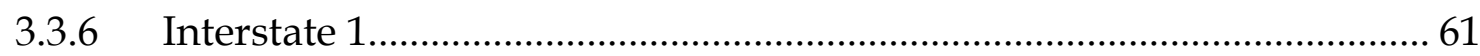

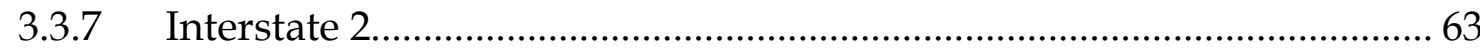

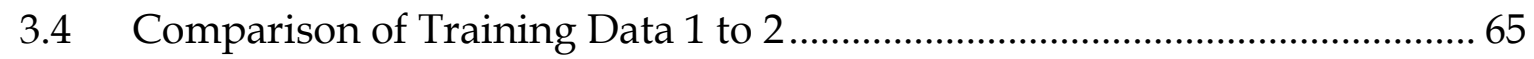

3.5 Elimination of Low Power Operation............................................................. 67

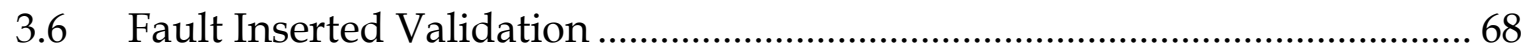

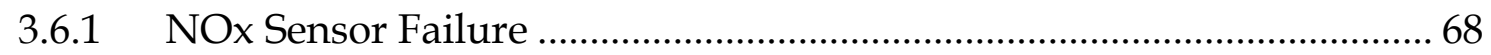

3.6.2 Boost Pressure Sensor Failure _................................................................. 71

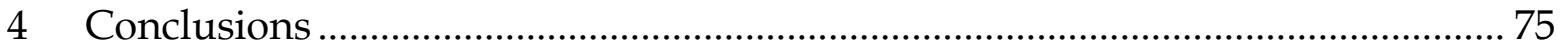

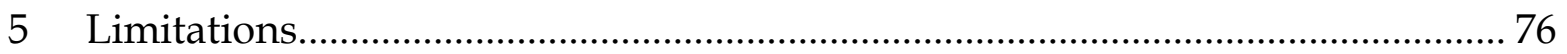

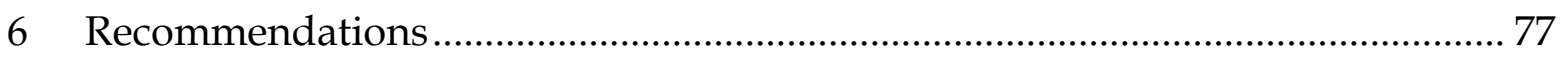

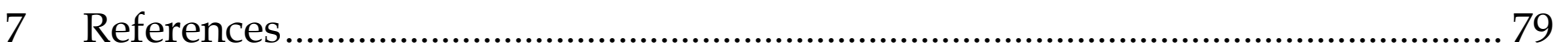

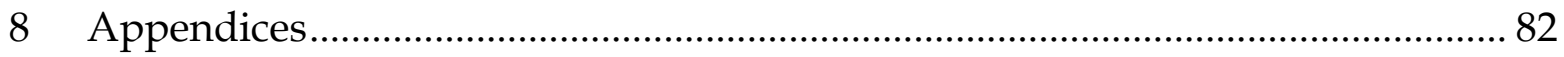




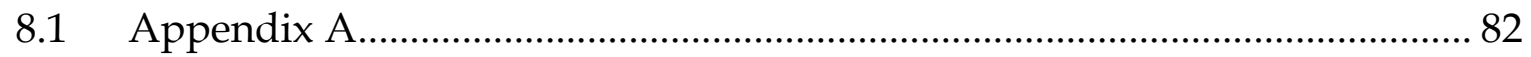

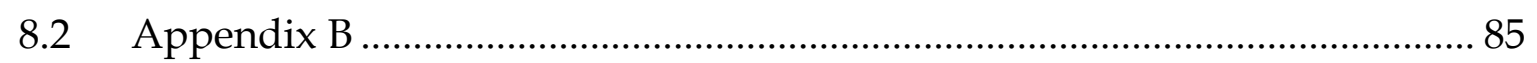

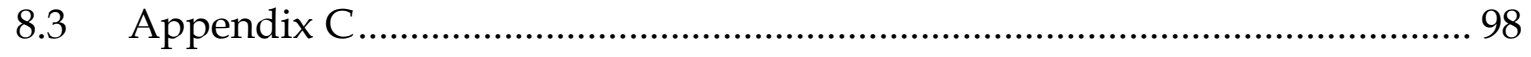

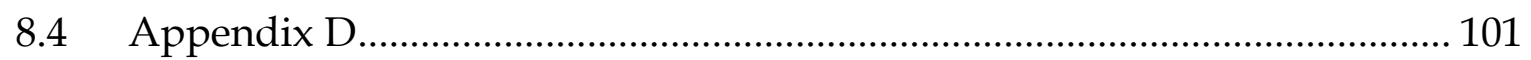

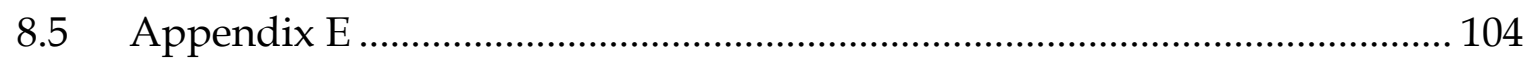

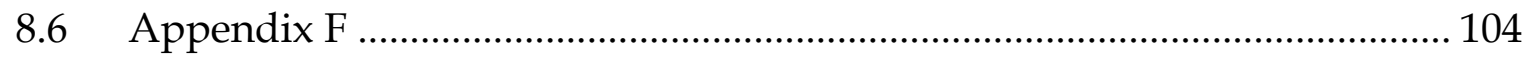

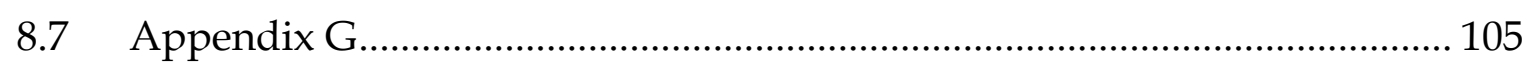

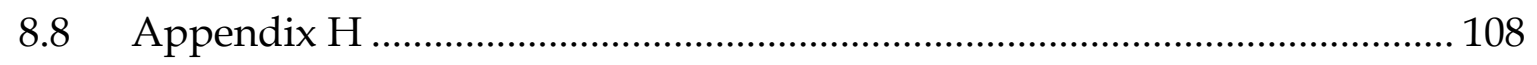

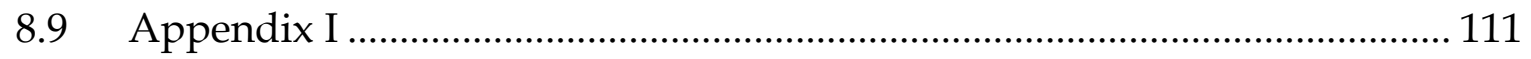




\section{LIST OF FIGURES}

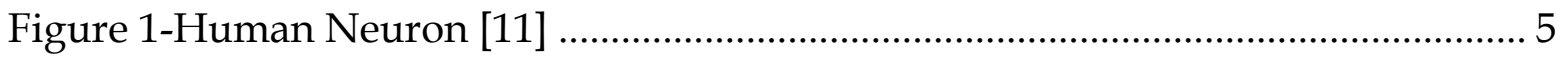

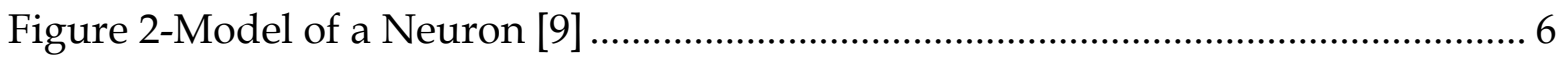

Figure 3-Diagram of Neural Network Layers ........................................................... 8

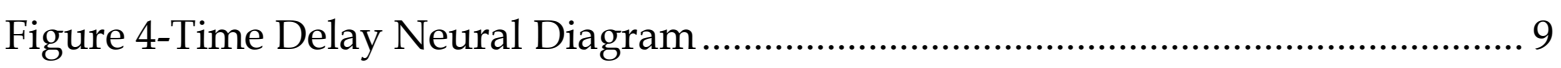

Figure 5-Speed Breakdown of Test Data …………………....................................... 15

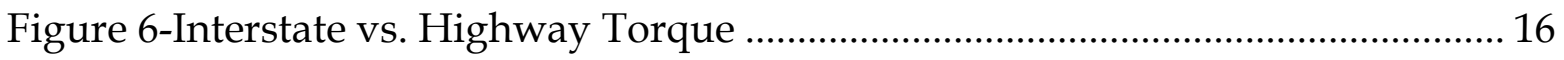

Figure 7-Neural Network Diagram....................................................................... 20

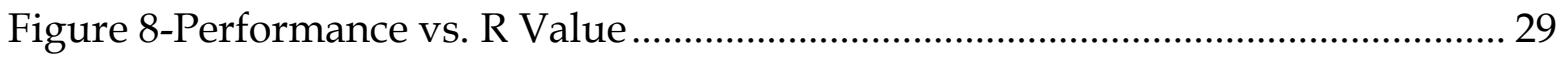

Figure 9-Training Output for Highway 1............................................................... 30

Figure 10-Training output for Combined Data ........................................................... 31

Figure 11-Scatterplot of Highway 2 Validation Test with NN Trained with Training

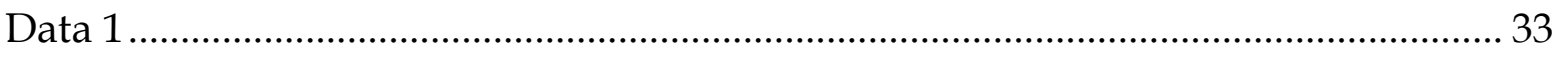

Figure 12-Actual vs. Predicted NOx for Highway 3 with 50 Point Smoothing......... 35

Figure 13-Enlarged Actual vs. Predicted for Highway 3 ............................................. 35

Figure 14- Scatterplot of Highway 3 Validation Test with NN Trained with Training

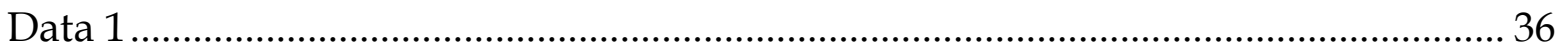

Figure 15- Scatterplot of Regional Validation Test with NN Trained with Training

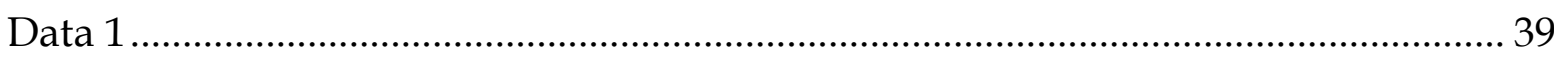

Figure 16- Scatterplot of Local Validation Test with NN Trained with Training Data

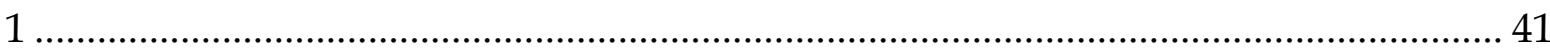

Figure 17- Scatterplot of Neardock Validation Test with NN Trained with Training Data 1

Figure 18- Scatterplot of Interstate 1 Validation Test with NN Trained with Training

Data 1 45

Figure 19- Scatterplot of Interstate 2 Validation Test with NN Trained with Training

Data 1 47

Figure 20-Scatterplot of Highway 2 Validation Test with NN Trained with Training Data 2

Figure 21- Scatterplot of Highway 3 Validation Test with NN Trained with Training Data 2

Figure 22- Scatterplot of Regional Validation Test with NN Trained with Training

Data 2 55

Figure 23- Scatterplot of Local Validation Test with NN Trained with Training Data 2 57 
Figure 24- Scatterplot of Neardock Validation Test with NN Trained with Training

Data 2 60

Figure 25- Scatterplot of Interstate 1 Validation Test with NN Trained with Training

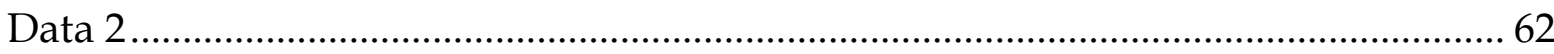

Figure 26- Scatterplot of Intersate 2 Validation Test with NN Trained with Training

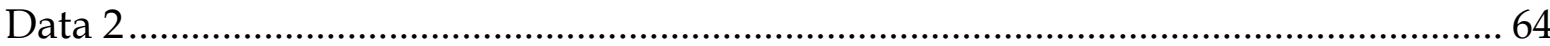

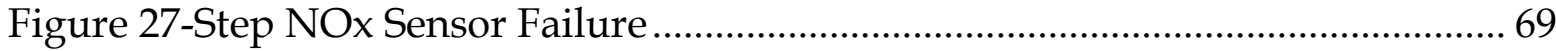

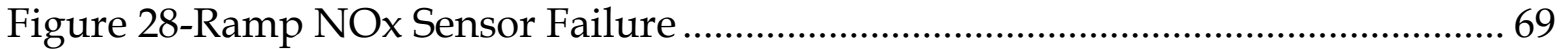

Figure 29-Square Wave NOx Sensor Failure .............................................................. 70

Figure 30-Boost Pressure Sensor Step Failure .......................................................... 72

Figure 31-Boost Pressure Sensor Ramp Failure........................................................ 72

Figure 32-Boost Pressure Sensor Square Function Failure ........................................... 73 


\section{LIST OF TABLES}

Table 1-1971 ARB Emissions Regulations ………….................................................. 2

Table 2-Percent Reduction of Pollutants from 1990 to 2015 [7]..................................... 3

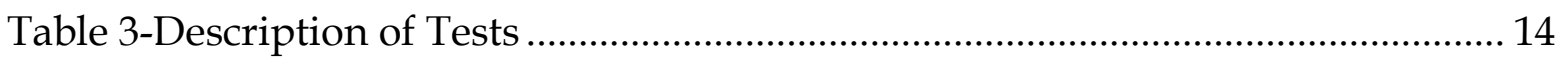

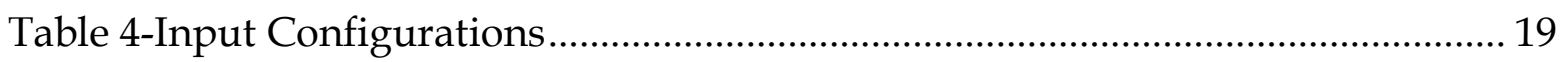

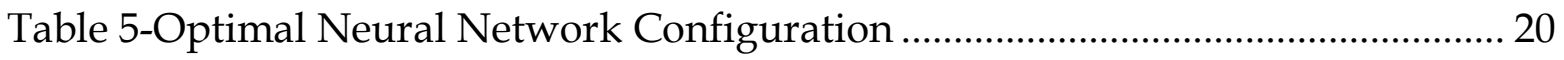

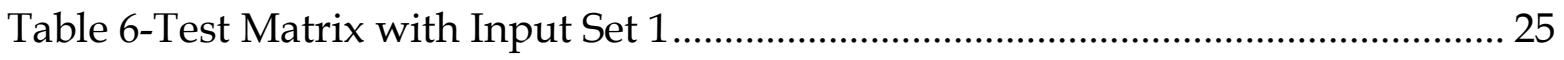

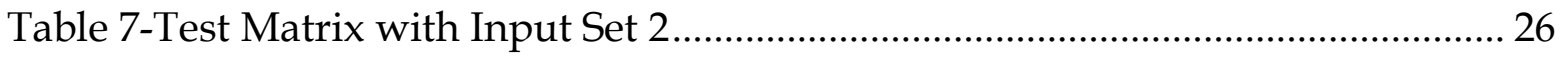

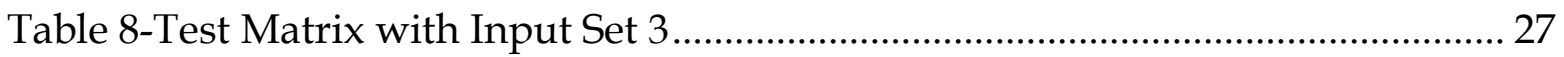

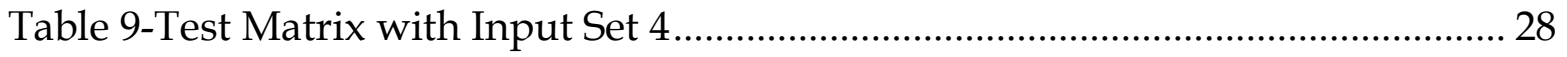

Table 10-Highway 2 Validation Test Errors when Trained with Training Data 1 .... 32

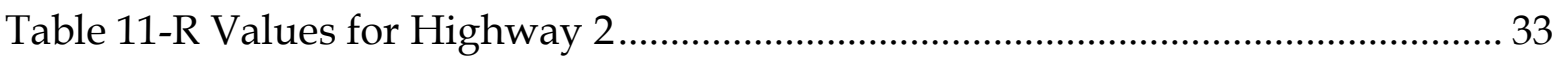

Table 12-Highway 2-200 Second Errors........................................................................ 34

Table 13- Highway 3 Validation Test Errors when Trained with Training Data 1 ... 36

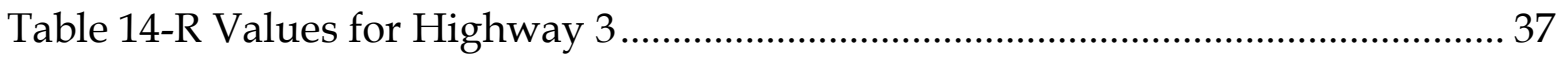

Table 15-Highway 3-200 Second Errors............................................................................ 38

Table 16- Regional Validation Test Errors when Trained with Training Data 1 ....... 39

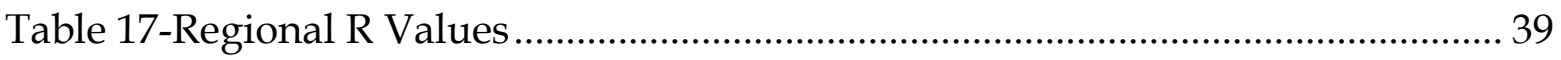

Table 18-Regional-200 Second Errors ......................................................................... 40

Table 19- Local Validation Test Errors when Trained with Training Data 1............. 41

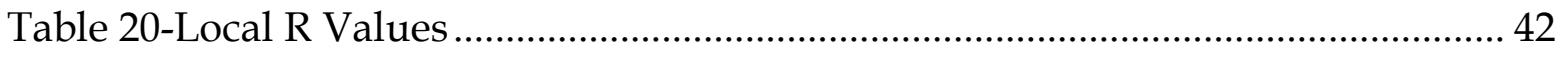

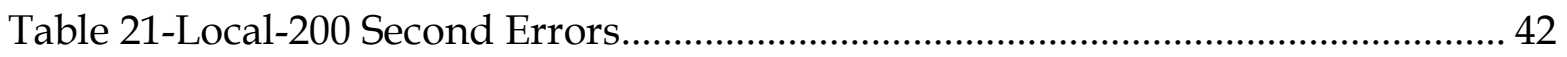

Table 22- Neardock Validation Test Errors when Trained with Training Data 1 ..... 43

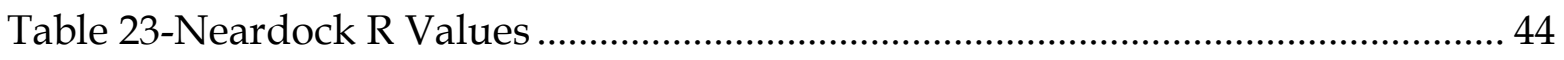

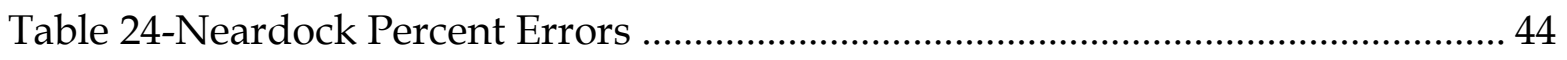

Table 25- Interstate 1 Validation Test Errors when Trained with Training Data 1... 45

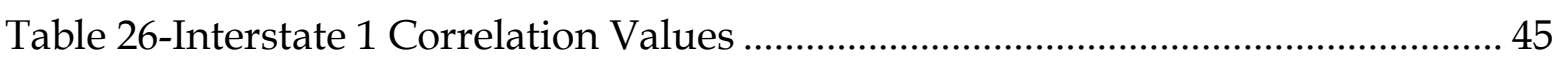

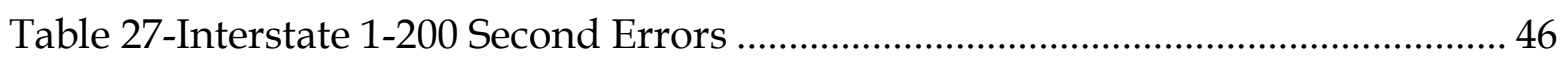

Table 28- Interstate 2 Validation Test Errors when Trained with Training Data 1... 46

Table 29-Interstate 2 Correlation Values ...................................................................... 47

Table 30-Interstate 2-200 Second Errors ......................................................................... 48

Table 31- Highway 2 Validation Test Errors when Trained with Training Data 2 ... 49

Table 32-Highway 2 Correlation Values .................................................................... 50

Table 33-Highway 2 Training Data 2-200 Second Errors.............................................. 51

Table 34- Highway 3 Validation Test Errors when Trained with Training Data 2 ... 52 
Table 35-Highway 3 Training Data 2 Correlation Values............................................... 53

Table 36-Highway 3 Training Data 2-200 Second Errors............................................... 54

Table 37- Regional Validation Test Errors when Trained with Training Data 2....... 55

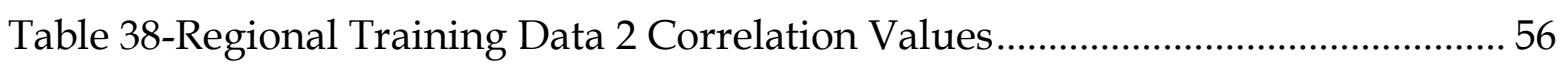

Table 39-Regional Training Data 2-200 Second Errors.................................................. 56

Table 40- Local Validation Test Errors when Trained with Training Data 2 ............. 57

Table 41-Local Training Data 2 Correlation Values....................................................... 58

Table 42-Local Training Data 2-200 Second Errors...................................................... 59

Table 43- Neardock Validation Test Errors when Trained with Training Data 2 .... 59

Table 44-Neardock Training Data 2 Correlation Values ............................................................ 60

Table 45-Neardock Training Data 2-200 Second Errors ................................................... 61

Table 46- Interstate 1 Validation Test Errors when Trained with Training Data 2... 61

Table 47-Interstate 1 Training Data 2 Correlation Values............................................. 62

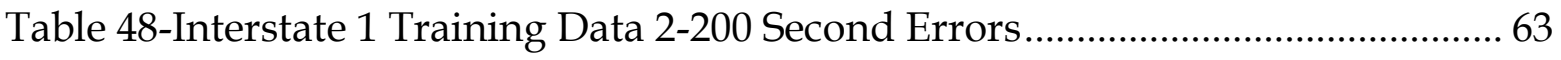

Table 49- Interstate 2 Validation Test Errors when Trained with Training Data 2... 63

Table 50-Interstate 2 Training Data 2 Correlation Values............................................ 64

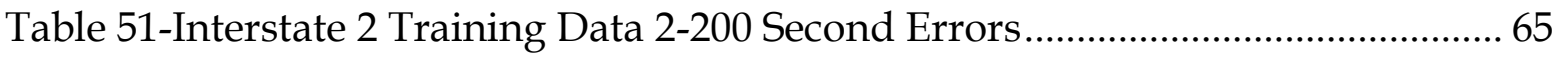

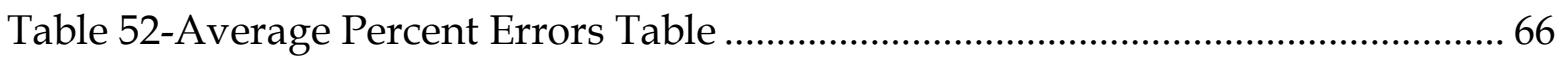

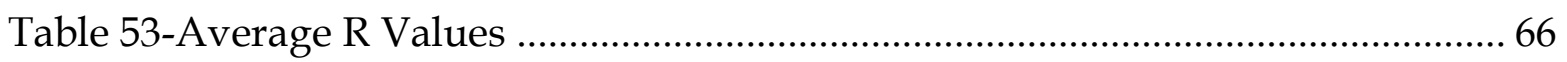

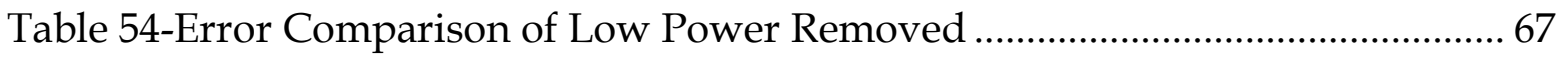

Table 55-R Value Comparison of Low Power Removed ............................................... 68

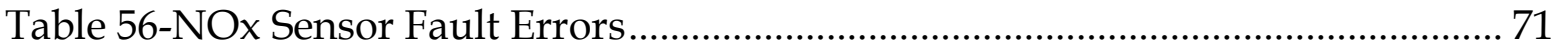

Table 57-Boost Pressure Sensor Failure Errors .......................................................... 74

Table 58-Vehicle Speed vs. Power Matrix ...................................................................... 77 


\section{SYMBOLS}

AARE

ANN

BFG

CAFEE

CARB

CGF, CGP, CGB

$\mathrm{CO}_{2}$

ECU

EGR

GD

GDm

IMEP

ITE

LM

MIL

MSE

NN

NAR

NARX

NOP

NOx

NRMSE

OBD

RMSE

SOI

VGT
Average Absolute Relative Error

Artificial Neural Network

Quasi-Newton

Center for Alternative Fuels, Engines and Emissions

California Air Resources Board

Conjugate Gradient Backpropagation

Carbon Dioxide

Engine Control Unit

Engine Gas Recirculation

Gradient Descent backpropagation

Gradient Descent backpropagation with momentum

Indicated Mean Effective Pressure

Indicated Thermal Efficiency

Levenberg-Marquardt

Malfunction Indicator Light

Mean Squared Error

Neural Network

Nonlinear Autoregressive

Nonlinear Autoregressive with External

Needle Opening Pressure

Oxides of Nitrogen

Normalized Root Mean Square Error

On-Board Diagnostics

Root Mean Squared Error

Start Of Injection

Variable Geometry Turbocharger 


\section{INTRODUCTION}

\subsection{Motivation}

The motivation for this study was to develop a model that could be used for the advancement of on-board diagnostics (OBD) for heavy-duty diesels. It can be seen in the California Code of Regulations [1] that fault limits decrease from 2010 to 2013, and will continue to decrease as new laws are published. This means that more accurate detection strategies must continue to be developed. The Code of Federal Regulations [2] states that a monitor must be able to make an evaluation within ten seconds and determine if there is a fault. The need for highly accurate, quick evaluation techniques is driving the development of new methods, like explored in this study.

\subsection{Objective}

The objective of this study is to lay the groundwork for the development of an OBD system using a virtual NOx sensor output and the readings of the engine out NOx sensor. If an accurate model can be developed to predict the NOx production in real time, this value can be compared to the NOx sensor reading to determine if there is either a problem with the NOx sensor or within one of the engine subsystems. A deviation in the prediction and NOx sensor reading may be used for fault detection and identification. The tasks to accomplish the overall objectives are listed below.

- Develop a well performing ANN

- Produce accurate NOx prediction with the ANN with on road data 
- Investigate fault detection of NOx sensor and engine subsystem sensors with the ANN

\subsection{BACKGROUND}

\subsubsection{DieSEL ENGINES AND EMISSIONS}

The California Air Resources Board (CARB) was created in 1967, after merging the California Motor Vehicle Pollution Control Board and the Bureau of Air Sanitation. There were some studies for the control of smoke and odor began in 1942, with the most significant paper from General Motors in 1956, but it was not until the enactment of Assembly Bill 357 that the Air Resources Board (ARB) was required to develop standards for carbon monoxide (CO), oxides of nitrogen (NOx), and hydrocarbon ( $\mathrm{HC}$ ) emissions. This bill stated that the standards had to be developed by January 1, 1971, and would begin to be enforced January 1, 1973 [3].

Table 1-1971 ARB Emissions Regulations

\begin{tabular}{|c|c|}
\hline \multicolumn{2}{|c|}{ 1971 ARB Emissions Regulations } \\
\hline HC+NOx & $16 \mathrm{~g} / \mathrm{hp}-\mathrm{hr}$ \\
\hline CO & $40 \mathrm{~g} / \mathrm{hp}-\mathrm{hr}$ \\
\hline
\end{tabular}

Ever since the first set of emissions regulations was set in 1971 (Table 1), there has been a continuous push for lower and lower emissions. Heavy duty engines emit four main types of pollutants; $\mathrm{CO}, \mathrm{HC}, \mathrm{NOx}$, and particulate matter (PM). $\mathrm{CO}$ is an odorless, colorless gas, that is poisonous to people and animals [4]. CO forms from incomplete combustion, as a result of less than ideal air and fuel mixture. [5]. CO can cause headache, dizziness, weakness, upset stomach, 
vomiting, chest pain, and confusion, and if enough is inhaled it can lead to a person passing out, or death [4]. HC are a result of unburnt fuel in the combustion process. $\mathrm{HC}$ are a mix of chemicals that not only contribute to the formation of ozone, but also are toxic to humans and animals and may be a cause of cancer [5]. NOx are produced when combustion temperature is in the 2500$3000 \mathrm{~K}$ temperature range [6]. NOx emissions directly contribute to the formation of ozone and have negative health effect on the respiratory system [5]. PM includes particles that are smaller than 2.5 microns and can pass through the nose and upper lungs that can cause irritation in the respiratory system [5].

Emissions regulations are important for the maintenance and improvement of air quality in the United States, and around the world. The Clean Air Act has reduced the levels of six pollutants: PM 2.5, ozone, lead, carbon monoxide, nitrogen dioxide, and sulfur dioxide [6]. Results show five of the six pollutants have reduced by an average of 70\% from 1970 to 2015 . The exclusion to this is ozone, which has only been reduced by $3 \%$. Table 2 shows the percentage reduction of pollutants in the air from 1990 to 2015 [7].

Table 2-Percent Reduction of Pollutants from 1990 to 2015 [7]

\begin{tabular}{|c|c|}
\hline \multicolumn{2}{|c|}{ Percent Reduction of Pollutants } \\
\hline Lead & $85 \%$ \\
\hline Carbon Monoxide & $84 \%$ \\
\hline Sulfur Dioxide & $67 \%$ \\
\hline Nitrogen Dioxide & $60 \%$ \\
\hline Ozone & $3 \%$ \\
\hline Fine Particle Concentrations & $37 \%$ \\
\hline Coarse Particle Concentration & $69 \%$ \\
\hline
\end{tabular}




\subsubsection{ON-BOARD DIAGNOSTICS}

On-board diagnostics is computer software that monitors emissions related components in an engine to ensure that everything is operating within the manufacture set range of values. The Clean Air Act Amendments (CAAA) of 1990 was the first OBD requirements [8]. These amendments required the monitoring of the catalyst and oxygen sensor at a minimum [8]. OBD is important to identify failure in any component of emissions control system that can result in higher levels of tailpipe emissions leading to non-compliant operation.

OBD for heavy-duty vehicles began in 2005 and were required for all vehicles over 14,000 pounds (gross vehicle weight rating) in 2013. These requirements include the illumination of a malfunction indicator light (MIL) and the storage of data in the ECU on the faulty component so that it can be downloaded. The primary reason OBD is needed is to regulate components over time. It was reported that the oldest twenty percent of vehicles cause sixty percent of pollution [9]. Other benefits include the early detection of failures and the elimination or unnecessary repairs [9].

CFR 40 86.1806-05.n.3.iii states "(iii) NOxsensors. If equipped, sensor deterioration or malfunction resulting in exhaust emissions exceeding any of the following levels: for 2007 through 2009 model years, 5 times the applicable PM standard, or 4 times the applicable NOx standard and, for 2010 through 2012 model years, 4 times the applicable PM standard, or the applicable NOX standard $0.6 \mathrm{~g} / \mathrm{mi}$ and, for 2013 and later model years, the applicable PM standard $0.04 \mathrm{~g} / \mathrm{mi}$, or the applicable NOX standard $0.3 \mathrm{~g} / \mathrm{mi}$." This rule declares that an OBD system must be able to capture a deteriorating NOx sensor, or any fault that may affect the NOx emissions. The CFR also states in section 86.1806-05.n.7 “(7)Performance of OBD functions. Any 
sensor or other component deterioration or malfunction which renders that sensor or component incapable of performing its function as part of the OBD system must be detected and identified on engines so equipped." This rule declares that the functionality of all sensors in the engine must perform within a tolerance to detect any problems in any subsystem of the engine.

\subsubsection{Artificial Neural Network}

An artificial neural network (ANN) is a computation system based on the function of the human brain. An ANN consists of parallel computing with interconnections of neurons. The connections between the neurons resemble the connectivity within the human brain as seen in Figure $1[11]$.

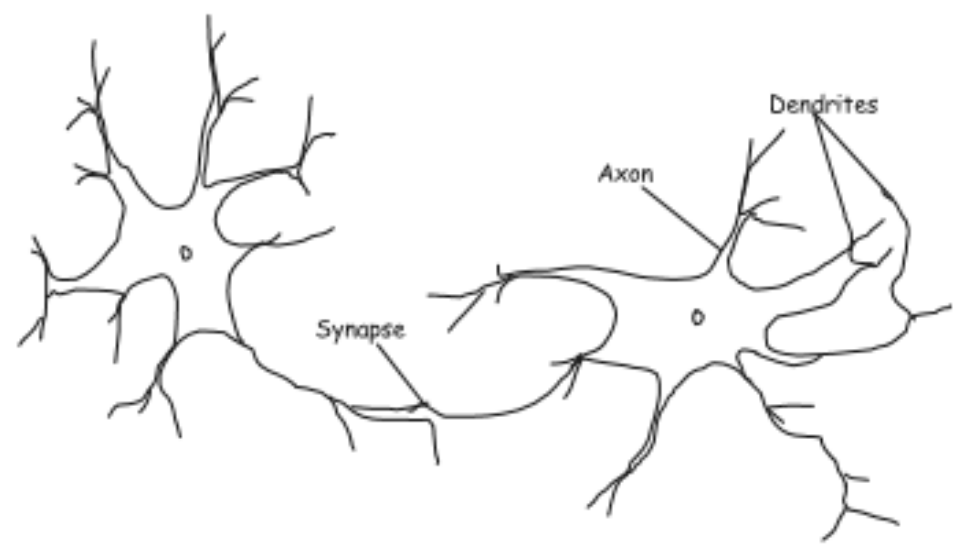

Figure 1-Human Neuron [11] 
Inputs Synaptic weights

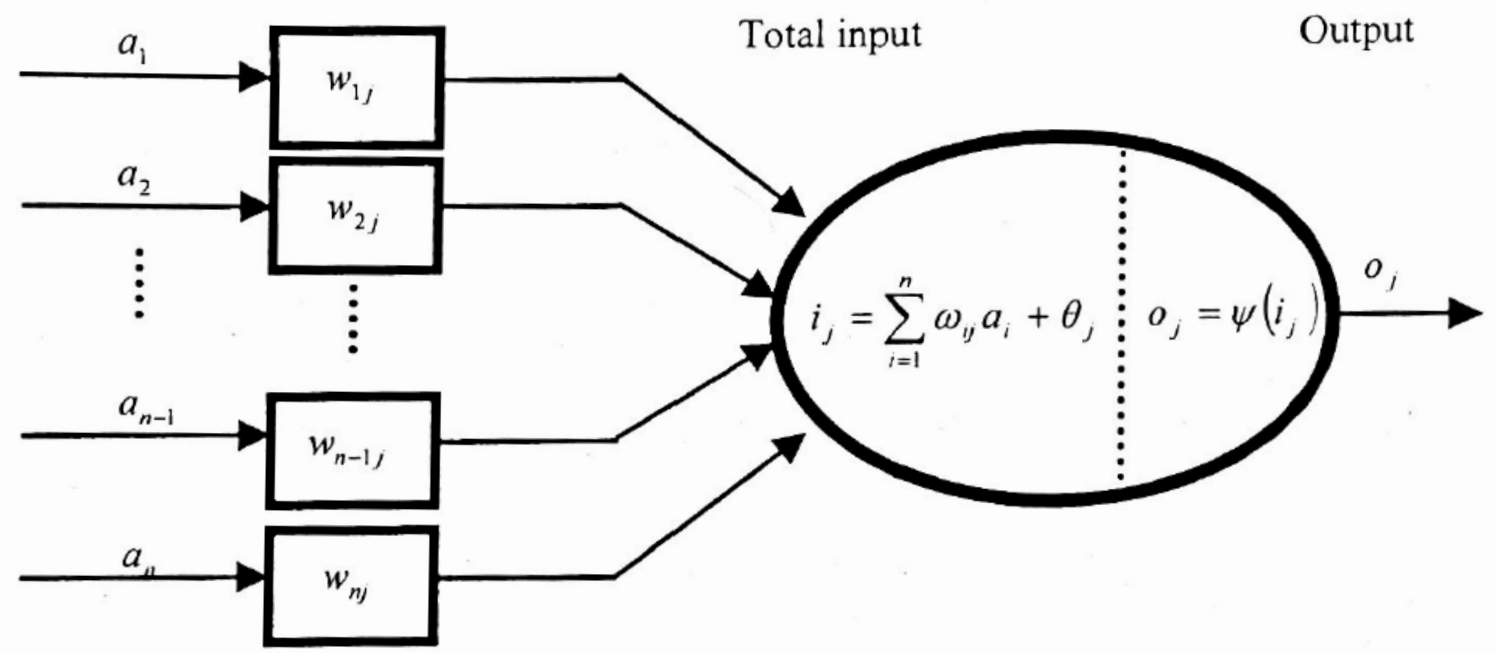

Axons of other Synapses Dendrites Axon neurons

Figure 2-Model of a Neuron [9]

Figure 2 shows the relation to the neuron in a neural network and how it compares to the biological neuron. The inputs $\left[a_{1}, a_{2}, \ldots a_{n}\right]$ are multiplied by weights $\left[w_{1}, w_{2}, \ldots w_{n}\right]$ in the synapses and then the summation of the inputs and weight are put through the transfer (or activation) function $\Psi$ in the neuron. The equation for the modified inputs and transfer function can be seen in Equation 1, where $o$ is the output and $\theta$ represents a bias value [12].

$$
o(t)=\Psi\left[\sum_{i=1}^{n} w_{i} a_{i}+\theta\right.
$$

Equation 1-Neuron Transfer Function

There are several transfer functions that can be used within a neural network. These include linear unbounded, unipolar binary, bipolar binary, unipolar semi-linear, bipolar semilinear, unipolar sigmoid, bipolar sigmoid, multimodal sigmoid, and radial basis. For this study, the hidden layer incorporated a bipolar sigmoid and the output layer incorporated a linear 
unbounded transfer function. These two functions are the default function configuration for a time series neural network within Matlab. The transfer functions can be changed to test for added accuracy, but this was not performed in this study. Equation 2 shows the bipolar sigmoid, and Equation 3 shows the linear unbounded, where $o_{j}$ is the output of neuron $j_{j}$, $i_{j}$ is the total input and $\alpha$ is a constant.

$$
\begin{gathered}
o_{j}(t)=\Psi\left[i_{j}(t)\right]=\tanh \left[\alpha i_{j}\right] \\
\text { Equation 2-Bipolar Sigmoid } \\
o_{j}(t)=\Psi\left[i_{j}(t)\right]=\alpha i_{j} \\
\text { Equation 3-Linear Unbounded }
\end{gathered}
$$

There are three different types of neurons within a neural network. There are input, output, and hidden neurons. A neural network can have multiple numbers of hidden layers. In this study, only one hidden layer was used. A single hidden layer is most common in neural networks, and is the default in the Matlab toolbox. The hidden layer size was an iterated variable tested for maximum performance that will be outlined in section 2.2.3. The number of neurons in the input and output layers are equal to the number of inputs and outputs used in the data. The number of neurons in the hidden layer is user defined. The number of hidden layer neurons was iterated for accuracy within this study. Figure 3 shows a diagram of the interaction of the three types of layers within the neural network [13]. 


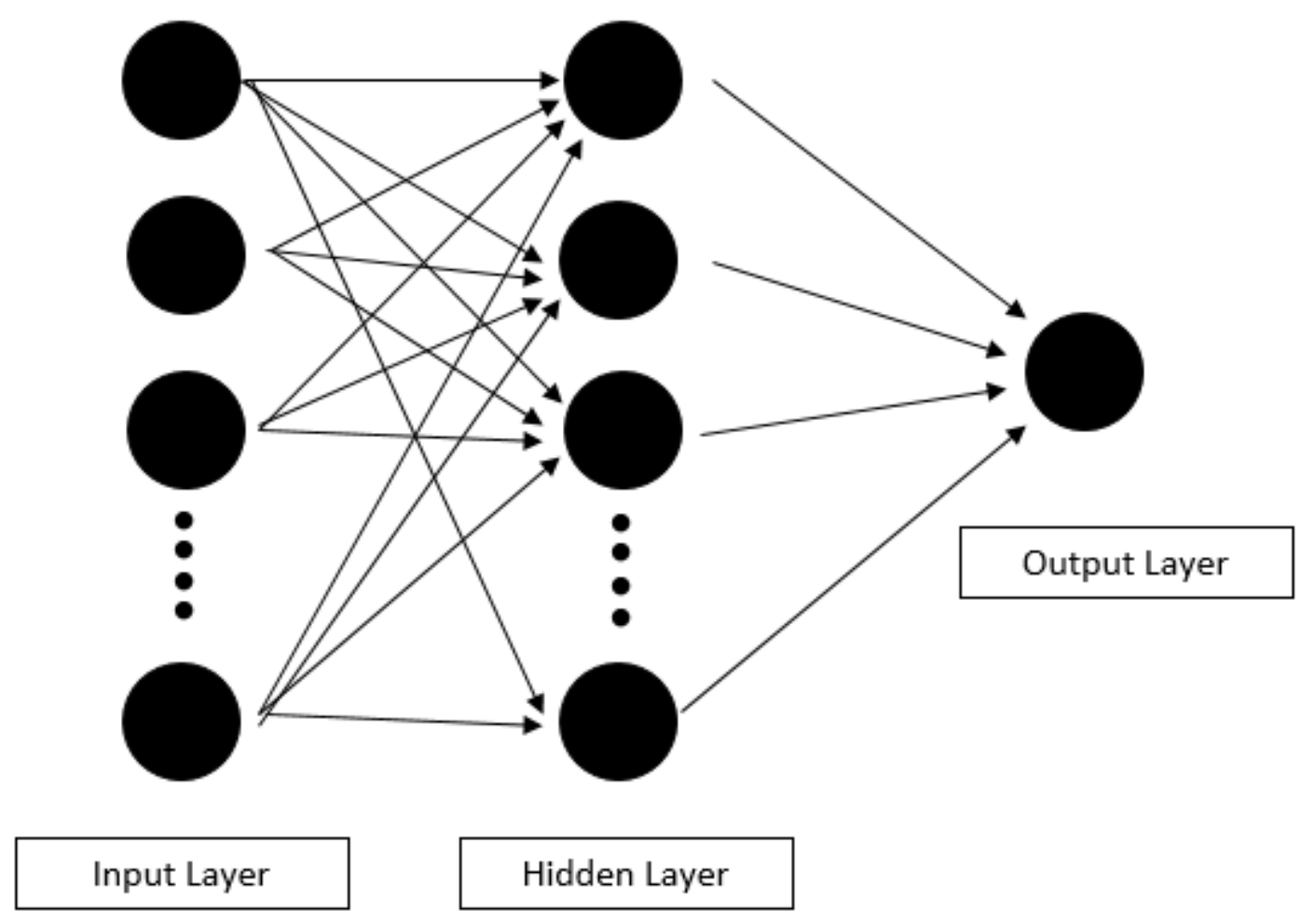

Figure 3-Diagram of Neural Network Layers

There are several different types of training algorithms used for neural networks. For this study, a backpropagation algorithm was used. A backpropagation algorithm uses two passes to adjust the weights and biases in the neural network. On the forward pass, the weights and biases are not adjusted. The difference between the output and the actual value is propagated backwards to update the weights and biases [14]. The two methods used in this study are Bayesian Regularization (BR) and Levenberg-Marquardt (LM). Both training methods utilize the Levenberg-Marquardt optimization, but Bayesian Regularization creates a network that generalizes well by minimizing a group of squared errors and weights [15]. The LevenbergMarquardt optimization algorithm can be seen in Equation 4, where $\mathrm{J}$ is the Jacobian matrix, $\mathrm{k}$ is the time step, $I$ is the identity matrix, $\mu$ is the learning rate, and e is the vector of network errors. [16] 


$$
w(k+1)=w(k)-\left[J_{k}^{T} J_{k}+\mu_{k} I\right]^{-1} J_{k}^{T} e_{k}
$$

Equation 4-Levenberg-Marquardt Optimization Algorithm

Another aspect of the neural network used in this study consists of the time delay. To better capture the operating state of the engine a time delay was incorporated into the neural network. The term time delay is used in the subject of an ANN to represent which data points are used to make the prediction. A two second time delay would describe the ANN using the data at the current time step and the previous two seconds of data to predict the outcome. In this study, the data was one hertz data so the input delay was equal to time in seconds. This technique is useful in transient situations where a history of the inputs is used for prediction. The time delay goes into the input layer of the neural network which incorporates the previous values to a defined number. Figure 4 shows the incorporation of the previous inputs into the current time step of the neural network [17].

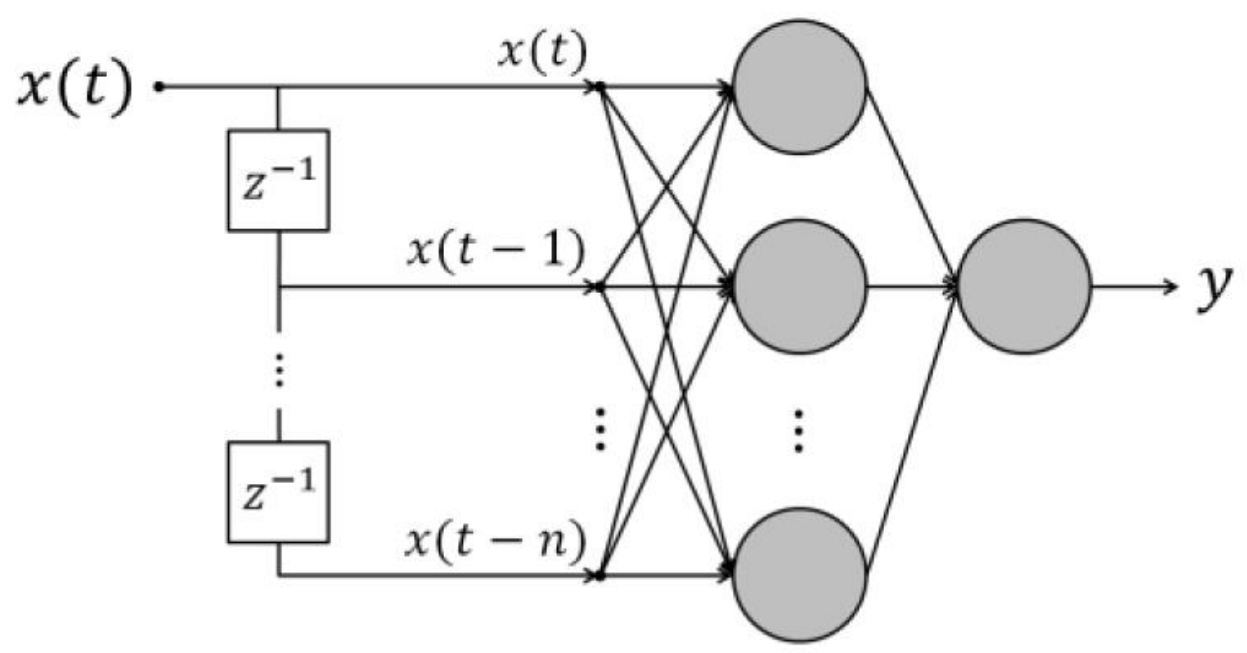

Figure 4-Time Delay Neural Diagram 


\subsection{LITERATURE REVIEW}

To develop a background of how neural networks have been used in the past, a few different studies were reviewed to evaluate the process of developing a neural network to predict NOx production from an engine.

In a study conducted by Anand et al. [18], they used a neural network to predict NOx emissions and efficiency of a spark ignited biogas engine. Anand et al. created a model using five inputs, which created two outputs. The inputs included, equivalence ratio, compression ratio, spark plug position, engine speed, and carbon dioxide $\left(\mathrm{CO}_{2}\right)$ fraction. Their neural network consisted of one hidden layer and varied the number of neurons from two to 12 . Anand et al. found that by varying the number of neurons in the hidden layer and measuring the Mean Squared Error (MSE), a hidden layer size of seven neurons provided the best results. While they did not disclose percentages in NOx prediction from their study, a MSE of 7.824e-05 was found from NOx prediction.

A second study by Gadallah et al. [14] used an artificial neural network to predict NOx production and indicated thermal efficiency (ITE) for a direct injection hydrogen engine equipped with water direct injection for NOx control. For the development of the ANN, Gadallah et al. used this study to examine the effects of training algorithms and transfer functions on performance of the neural network. All training algorithms used were backpropagating. The training algorithms included Gradient Descent backpropagation (GD), Gradient Descent backpropagation with momentum (GDm), Conjugate Gradient backpropagation (CGF), (CGP), (CGB), Quasi-Newton (BFG), and Levenberg-Marquardt (LM). Gadallah et al. also used four transfer functions including logistic sigmoid, symmetric logistic, hyperbolic tangent sigmoid, and linear. 
To begin their testing, a training algorithm was selected and the number of neurons in the hidden layer was held constant at four. The transfer functions were then iterated for the hidden layer and output layer. The root mean squared error (RMSE) and average absolute relative error (AARE) were recorded for each iteration. The procedure was then repeated varying the number of hidden layer nodes from four to 40 . The combination that gives the lowest RMSE and AARE was considered for the best possible solution. This process was conducted for every training algorithm.

Six inputs: equivalence ratio, indicated mean effective pressure (IMEP), hydrogen start of injection (SOI), spark ignition timing, water injection quantity, and water injection timing, were used. The output of the neural network included NOx and indicated thermal efficiency (ITE). The results showed that the Levenberg-Marquardt algorithm, 18 neurons in the hidden layer, hyperbolic tangent sigmoid and linear for the hidden and output layers, respectively, gave the best prediction results.

In another study, Huayi et al. [17] used a multilayer neural network to predict NOx emissions and smoke production in a light-duty turbocharged diesel engine. Huayi et al. considered NOx production at steady state and transient conditions. For steady state prediction engine speed, fueling, main injection timing, injection pressure, intake pressure, intake temperature, and mass air flow were selected. To predict NOx in transient operation engine speed, fueling, intake pressure, intake temperature, mass air flow, and delays of $0.2,0.4,0.6,0.8$, and 1 second for fueling, intake pressure, intake temperature, and mass air flow were selected. This resulted in 25 total inputs for transient NOx prediction. For training of the neural network, the Levenberg-Marquardt method was used. The neural network used incorporated two hidden 
layers. The neural network was able to predict NOx production with a correlation coefficient of 0.9978.

This study predicted NOx emissions for a two liter, four cylinder, common rail, turbocharged, diesel engine [19]. For the neural network, a total of six inputs were used, including engine speed, engine torque, injection timing, air flow rate, rail pressure, and oil temperature. Zhang et al. used a nonlinear tangent sigmoid function within the hidden layer and a weighted summation within the output layer. The number of neurons in the hidden layer were varied between one and twenty. It was found that with fourteen neurons in the hidden layer, the network showed the best predictions. They concluded that the Normalized Root Mean Square error (NRMSE) was found to be between 5.11 and 8.12 percent difference in predicted versus actual NOx for the four drive cycles tested.

The final study reviewed was based on the development of a neural network for the prediction of NOx from a standard six cylinder, 12 liter heavy-duty EURO-2 engine, at transient operation [20]. The inputs used for the neural network included, engine speed, engine torque, intake pressure, and intake temperature. The intake variables incorporated a time delay different for each variable. Engine speed was used at the current time step and the previous time step. Engine torque included the current time step and the four previous time steps. Intake air pressure was used for the current time step and two previous steps, and no delays were used for the intake air temperature. The number of hidden layers was iterated between zero, one, or two, and the number of neurons was varied between eight and 40 . Krijnsen et al. found that the optimal number of hidden layers was one, and the number of neurons within the hidden layer was not clear. With the number of neurons between 20 and 40, the network produced results 
between 5-5.5 percent difference between predicted and measured NOx. It was decided that 30 neurons would be used for the study. Upon running the validation data, it was found that there was an average of 6.7 percent error between predicted and actual values for 291 measurements.

To investigate the deterioration of a NOx sensor over time, a study conducted by Orban et al. was reviewed [21]. In this study 25 NOx sensors were exposed to exhaust gas for 6000 hours. The sensors underwent calibration checks at the beginning of the test, and after every 2000 hours. The results concluded that depending on the location of the NOx sensor, the degradation of the NOx sensor was between two and 20 percent. This shows the need for a monitor to evaluate the performance of the NOx sensor over the life of the vehicle to ensure accurate NOx readings.

In a study conducted by the US Army Ordnance Center \& School and Pacific Northwest Laboratory [22], they explored the use of a neural network to obtain real-time fault diagnostics with more accurate results. In this study derivatives of some input parameter were computed and the neural network functioned in real time within the ECU. This shows the low level of computational intensity of a neural network based on the small number of basic functions used to make the prediction. This study concluded that the use of a neural network would save time and improve diagnostic capabilities. 


\section{EXPERIMENTAL DATA AND PROCEDURES}

\subsection{DATA}

\subsubsection{TEST DATA}

All the data used for this study was collected by the Center for Alternative Fuels, Engines and Emissions (CAFEE) at West Virginia University. The data was recorded during in-use vehicle operation in California in 2015. The vehicle used for testing was a 2014 Freightliner implemented with a 2013 ISX15 engine, and an Allison ten speed manual transmission. The testing utilized the same driver for all operation of the truck. The data was broken into eight data sets, dependent on operating states. All cold start and regen operation were excluded for the data used. The data set names and descriptions can be found in Table 3.

Table 3-Description of Tests

\begin{tabular}{|c|c|c|}
\hline Test Name & Route & Duration \\
\hline Highway 1 & $\begin{array}{c}\text { Ontario to Sacramento part } \\
1\end{array}$ & $5000 \mathrm{sec}$. \\
\hline Highway 2 & $\begin{array}{c}\text { Ontario to Sacramento part } \\
2\end{array}$ & $5100 \mathrm{sec}$. \\
\hline Highway 3 & Ontario to Sacramento part & $5500 \mathrm{sec}$ \\
\hline Interstate 1 & Irvine Route part 1 & $2850 \mathrm{sec}$. \\
\hline Interstate 2 & Irvine Route part 2 & $2750 \mathrm{sec}$. \\
\hline Regional & LA to Ontario & $3750 \mathrm{sec}$. \\
\hline Local & Intermodal Way & $4500 \mathrm{sec}$. \\
\hline Near Dock & Intermodal Way & $1300 \mathrm{sec}$. \\
\hline
\end{tabular}




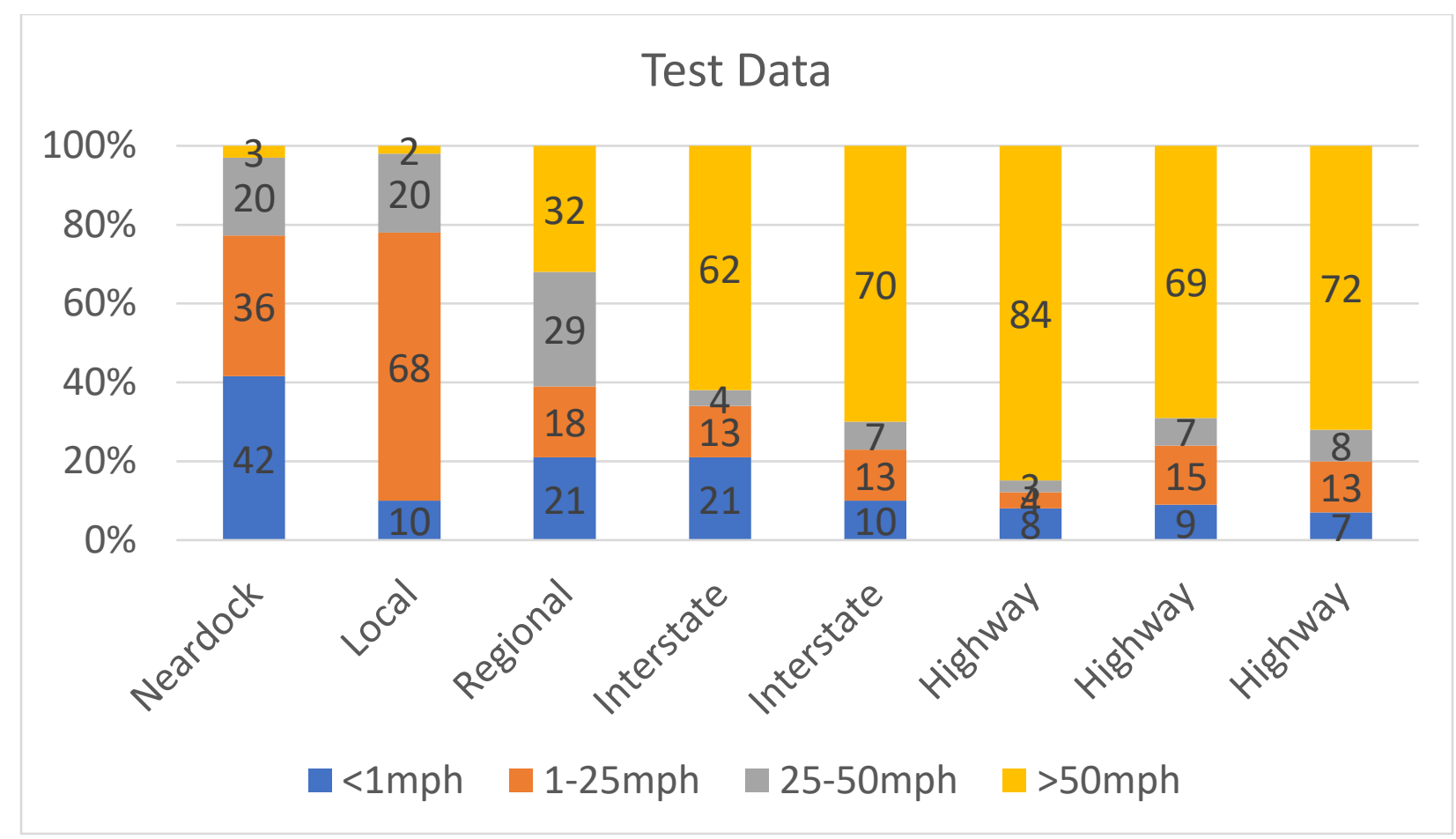

Figure 5-Speed Breakdown of Test Data

As shown in Figure 5 the test data sets contain the percentages of speed breakdown for the data sets. These four ranges were arbitrarily selected to represent idle, city, suburban, and highway driving conditions. These locations consist of vastly different vehicle operations based on percentage of time spent within each speed range. This provided the opportunity to observe how the model would perform during all operating speeds of the vehicle. 


\subsubsection{InTERSTATE VS. HighWAY Data COMParison}

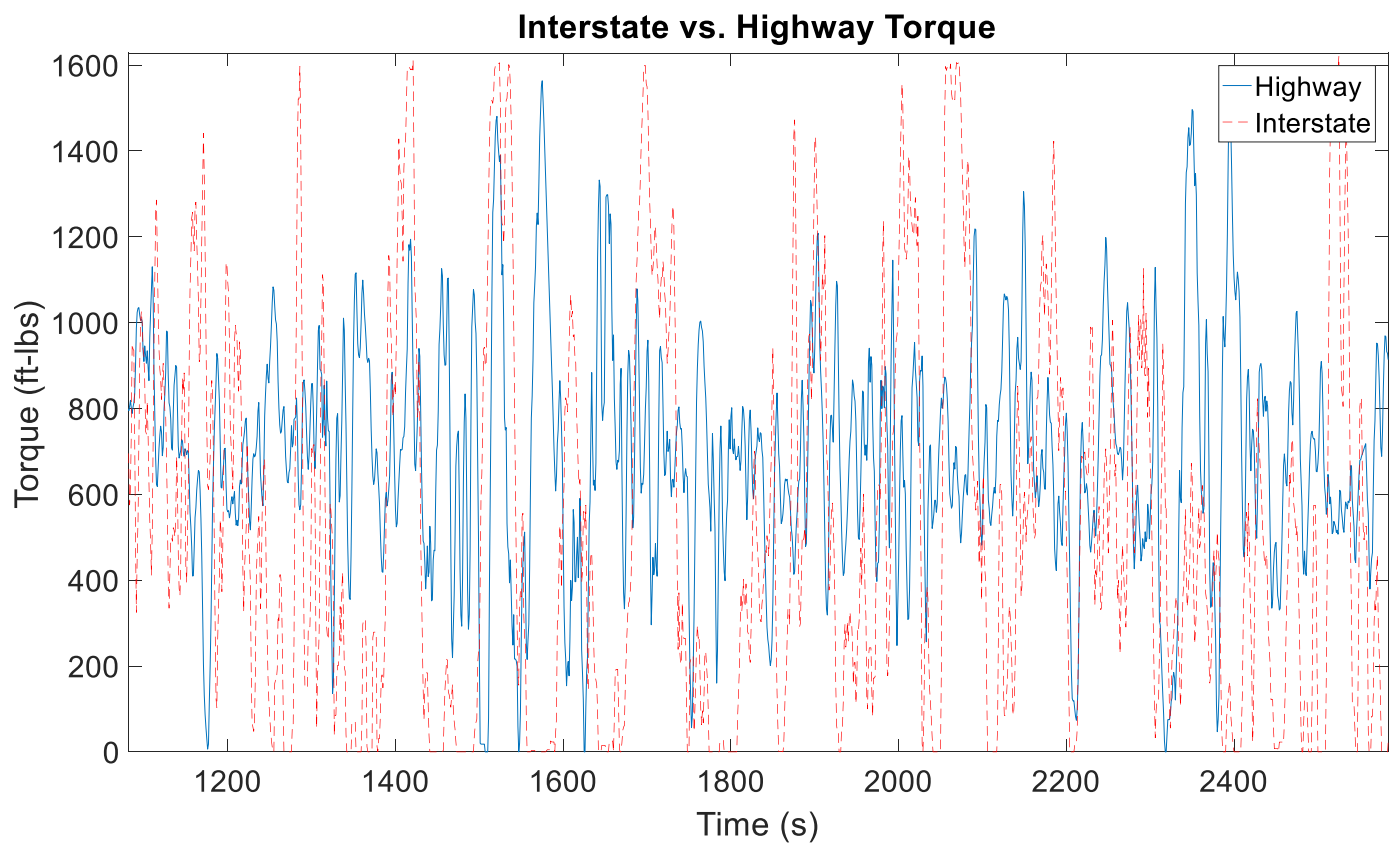

Figure 6-Interstate vs. Highway Torque

Although the interstate and highway speed breakdown in Figure 5 resemble each other, can be seen in Figure 6 that torque demand is much different. The torque in the interstate data has larger variations throughout the test than the highway. This means that even though the interstate data resembles highway data from the speed percentage breakdown, the NOx production is different due to the low speed and idle portions mixed throughout the dataset. The low speed and idle portions, mixed throughout the test, create large portions of braking and hard acceleration, which can be seen in Figure 6. With the increased torque demand and engine speed, the NOx production from the engine is much different than that of highway operation.

One objective of this study was to monitor the subsystems of the engine and determine if there was an operating problem. To properly achieve this objective the NOx production for a properly running engine was desired for comparison. All the data collected was from a properly 
running engine with no known faults, and excluded operation during cold starts, or when a sensor was not reading properly.

\subsubsection{Combined Training Data SeT}

For an alternative training method, a combined data set was created. This combined data set consisted of a portion of four different tests. The combined training data set was 3500 seconds of data. The first 500 seconds came from the 500-1000 second portion of the Neardock data set. The next 1000 seconds came from the 1000-2000 second portion of the Regional data set. The following 1000 seconds came from the 1000-2000 second portion of the Interstate 1 data set. The last 1000 seconds came from the 1000-2000 second portion of the Highway 1 data set. This combined data set captured a slice of vehicle operation at many of the locations.

\subsection{TRAINING}

\subsubsection{Model Selection}

There are a variety of types of ANNs. For this study, a prediction of transient data was desired; therefore, a time series type of ANN was required. A Nonlinear Input-Output neural network was chosen. A Nonlinear Autoregressive with External (Exogenous) Input (NARX) or Nonlinear Autoregressive (NAR) type of time series ANN could not be used because of the use of a feedback loop. The feedback loop would enable the model to learn deterioration and drift of the NOx sensor. One objective of this study was to be able to determine NOx sensor failure, which could be in the form of a deterioration drift over time from the true emissions production. With the use of a feedback loop, the neural network would continually learn the output value, meaning that if the sensor developed a drift in either the positive or negative direction, the neural network 
would learn this and adjust accordingly. Therefore, the neural network would begin to predict an offset value, eliminating differentiation between the prediction and offset drifted value.

\subsubsection{INPUTS}

The inputs to the network needed to serve two purposes. The first purpose was to give a sufficient amount of detail to make an accurate prediction of NOx production. The second purpose of the chosen inputs was to be a gateway that could be used to monitor engine subsystems. Since proprietary manufacturer data was not available, channels that could represent the amount of Exhaust Gas Recirculation (EGR), Variable Geometry Turbocharger (VGT) position, combustion characteristics, and operating states were desired. This needed to be done with publicly accessible data through the OBD port which was all that was available for this study. For example, intake temperature could be used for detection of changes in EGR position since an increase in EGR flow would cause a change in intake temperature. Eight ECU parameters were chosen to be used in the neural network. The eight parameters chosen were engine speed, engine torque, fuel rate, intake temperature, boost pressure, exhaust temperature, coolant temperature, and oil pressure. The inputs in this study were all engine based, and did not incorporated vehicle parameters. All inputs were normalized between either 0 and 1 or -1 and 1 , depending on the nature of the variable.

To further increase the prediction abilities of the neural network, additional forms of the engine speed and engine torque parameters were created. The first and second derivatives along with five-point smoothing of the speed and torque were created. The smoothing was completed using the 'smooth' function inside Matlab. The parameters were smoothed due to the high frequency transients throughout the data. The first and second derivatives of the engine speed 
and torque parameters were helpful in capturing peaks of NOx production during sudden braking/acceleration occurrences.

Table 4-Input Configurations

\begin{tabular}{|l|l|l|l|}
\hline \multicolumn{5}{|c|}{ Input Configurations } \\
\hline Input Configuration 1 & Input Configuration 2 & Input Configuration 3 & Input Configuration 4 \\
\hline Engine Speed & Engine Speed & Engine Speed & Engine Speed \\
\hline Torque & Torque & Torque & Torque \\
\hline Fuel Rate & Fuel Rate & Fuel Rate & Fuel Rate \\
\hline Intake Temperature & Intake Temperature & Intake Temperature & Intake Temperature \\
\hline Boost Pressure & Boost Pressure & Boost Pressure & Boost Pressure \\
\hline Exhaust Temperature & Exhaust Temperature & Exhaust Temperature & Exhaust Temperature \\
\hline Coolant Temperature & Coolant Temperature & Coolant Temperature & Coolant Temperature \\
\hline Oil Pressure & Oil Pressure & Oil Pressure & Oil Pressure \\
\hline & First Derivative of & First Derivative of & $\begin{array}{l}\text { First Derivative of } \\
\text { Engine Speed }\end{array}$ \\
& Engine Speed & Engine Speed & $\begin{array}{l}\text { Second Derivative of } \\
\text { Engine Speed }\end{array}$ \\
\hline & First Derivative of & Second Derivative of \\
Engine Speed & $\begin{array}{l}\text { First Derivative of } \\
\text { Torque }\end{array}$ \\
\hline & & First Derivative of \\
& & Torque & $\begin{array}{l}\text { Second Derivative of } \\
\text { Torque }\end{array}$ \\
\hline & & & $\begin{array}{l}\text { Second Derivative of } \\
\text { Torque }\end{array}$ \\
\hline & & $\begin{array}{l}\text { 5-point Smoothing of } \\
\text { Engine Speed }\end{array}$ \\
\hline & & $\begin{array}{l}\text { 5-point Smoothing of } \\
\text { Torque }\end{array}$ \\
\hline
\end{tabular}

\subsubsection{MOdEL OPTIMIZATION}

To find the best neural network strategy, a test matrix was constructed. The test matrix iterated different input configurations, delay times, hidden layer size, and computational type. The Pearson Correlation Coefficient ( $R$ value) [18] and performance in the form of Mean Squared Error (MSE) were recorded for each of the tests. The R value describes the correlation between the predicted and actual values. An $\mathrm{R}$ value of one describes a perfect match between a predicted value and the measured value. $R$ values in between zero and one are broken 
into five categories, $0-0.19$ is very weak, $0.2-0.39$ is weak, $0.4-0.59$ is moderate, $0.6-0.79$ is strong, and 0.8-1.0 is very strong [23].

$$
R=\frac{\sum(x-\bar{x})(y-\bar{y})}{\sqrt{\sum(x-\bar{x})^{2} \sum(y-\bar{y})^{2}}}
$$

Equation 5-Pearson Correlation Coefficient ( $R$ Value)

$$
\mathrm{MSE}=\sum_{i=1}^{N} \frac{\left(Y_{i}^{\prime}-Y_{i}\right)^{2}}{N},
$$

Equation 6-Mean Squared Error

These results can be seen in the Neural Network Development section of the results. The $R$ value and performance were then plotted against each other for evaluation to select the optimal neural network combination. The optimal combination can be seen in Table 5. Figure 7 shows the layout of neural network.

Table 5-Optimal Neural Network Configuration

\begin{tabular}{|c|c|c|c|}
\hline Inputs & Hidden Layer Size & Input Delay & Training Algorithm \\
\hline Input Configuration 3 & 20 & 2 & Bayesian Regularization \\
\hline
\end{tabular}

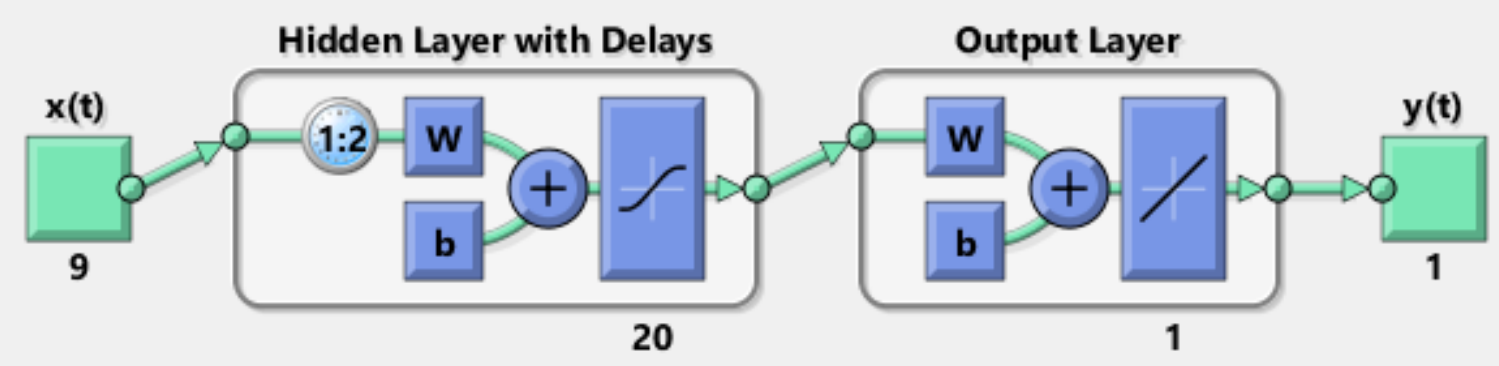

Figure 7-Neural Network Diagram 


\subsection{VALIDATION}

After an ANN combination was selected, it could then be trained. For this study two different sets of training data were used. The first set of training data was Highway 1 , and the second set was the combined training data. After the network was trained it could be used to predict NOx on the other data sets. For validation using the first training data set, the Highway 1 data was used for training and then validated against all the other data sets which can be seen in Table 3 . This allowed for the complete separation of training and validation data. For validation when the ANN was trained with the combined training data, there was some overlap. The highway portion of the combined training data was excluded since the Highway 1 data was not used for validation, but the other portions had some overlap. Due to the limited amount of data for this study, the Neardock, Regional, and Interstate validation tests, included some overlap with the combined training data. The predicted values could then be compared to the actual data and evaluated. A summed percent error between the predicted and actual values were computed for each of the data sets. A time segment of 200 seconds was arbitrarily selected to sum the actual and predicted NOx values to use for further performance comparison in terms of percent error. Since a neural network prediction will change slightly each time it is trained, the validation tests were performed five times on each data set, retraining the network before each iteration. The validated was run five times to obtain enough values in order to calculate an average representative of the performance with each data set.

\subsection{EXPLORATION OF POOR PREDICTION AREAS}

One hypothesis was that the ANN performed poorly at low power operating states. To explore this hypothesis, all data points in the bottom 20 percent of maximum power were 
eliminated from the data sets to be evaluated. The average values of percent error and $\mathrm{R}$ for the five iterations of validation testing with and without the low power points were recorded for evaluation.

\subsection{OBD DEVELOPMENT}

Once a neural network had been created, an investigation could be conducted to determine if and when there was a problem with the NOx sensor or one of the ANN input parameters. To begin the fault detection investigation, fault inserted data sets were created to be run against the ANN. Three different types of sensor failure methods were simulated. These three failure methods include a step, ramp, and square wave failure. A ramp failure could simulate a deteriorating NOx sensor that has a reading drifting from the actual value. The step and square wave failures can simulate the failure or loss of communication from the NOx sensor to the vehicle ECU. To investigate the use of the neural network to be able to detect these failures the neural network trained with training data 1 , and the Highway 3 data set was used. This combination of neural network and validation data was used since it provided the most accurate prediction. The Highway 3 data set was manipulated to incorporate faults, of the NOx sensor and boost pressure sensor, and compared to the nominal NOx values.

\subsubsection{NOX SENSOR FAILURE}

For fault detection of the NOx sensor, each of the three failure types had to be simulated in the NOx sensor readings. For the step failure, the value was untouched for the first half of the test and then would step to a value forty percent above nominal value. For the second failure method, a ramp failure was simulated by increasing the value of the reading gradually over the length of the test. The final value would be twenty-five percent above nominal value. The third 
failure method would represent the ECU losing connection to the NOx sensor every 400 seconds for 400 seconds. The 400 seconds was arbitrarily chosen just to show the fluctuation of the signal several times over the length of the data set. To simulate this failure method, the NOx values were forced to zero alternating on and off every 400 seconds.

\subsubsection{Boost Pressure Sensor FaILURE}

The objective of this study was to not only investigate fault detection of the NOx sensor, but to also detect a fault in an engine subsystem. There are sensors in the engine that can change the NOx production if the reading is not correct. Some of these sensors include, the coolant temperature sensor, exhaust temperature, fuel rate, and intake temperature. To investigate the detection of a problem in an engine subsystem the boost pressure channel was selected to be manipulated for this study. The three types of sensor failures were implemented into the boost pressure signal to observe how the neural network prediction would be affected. Like the simulated NOx sensor failure, the boost pressure signal underwent the step, ramp, and square wave function in identical offsets. 


\section{RESULTS AND DISCUSSION}

\subsection{Neural Network DeVelopment}

As discussed in the procedures for optimizing the neural network in Section 2.2.3, a test

matrix was constructed to test for the optimal design parameters. The resulting Performance and

$R$ value can be seen in Tables 6, 7, 8, and 9 . 
Table 6-Test Matrix with Input Set 1

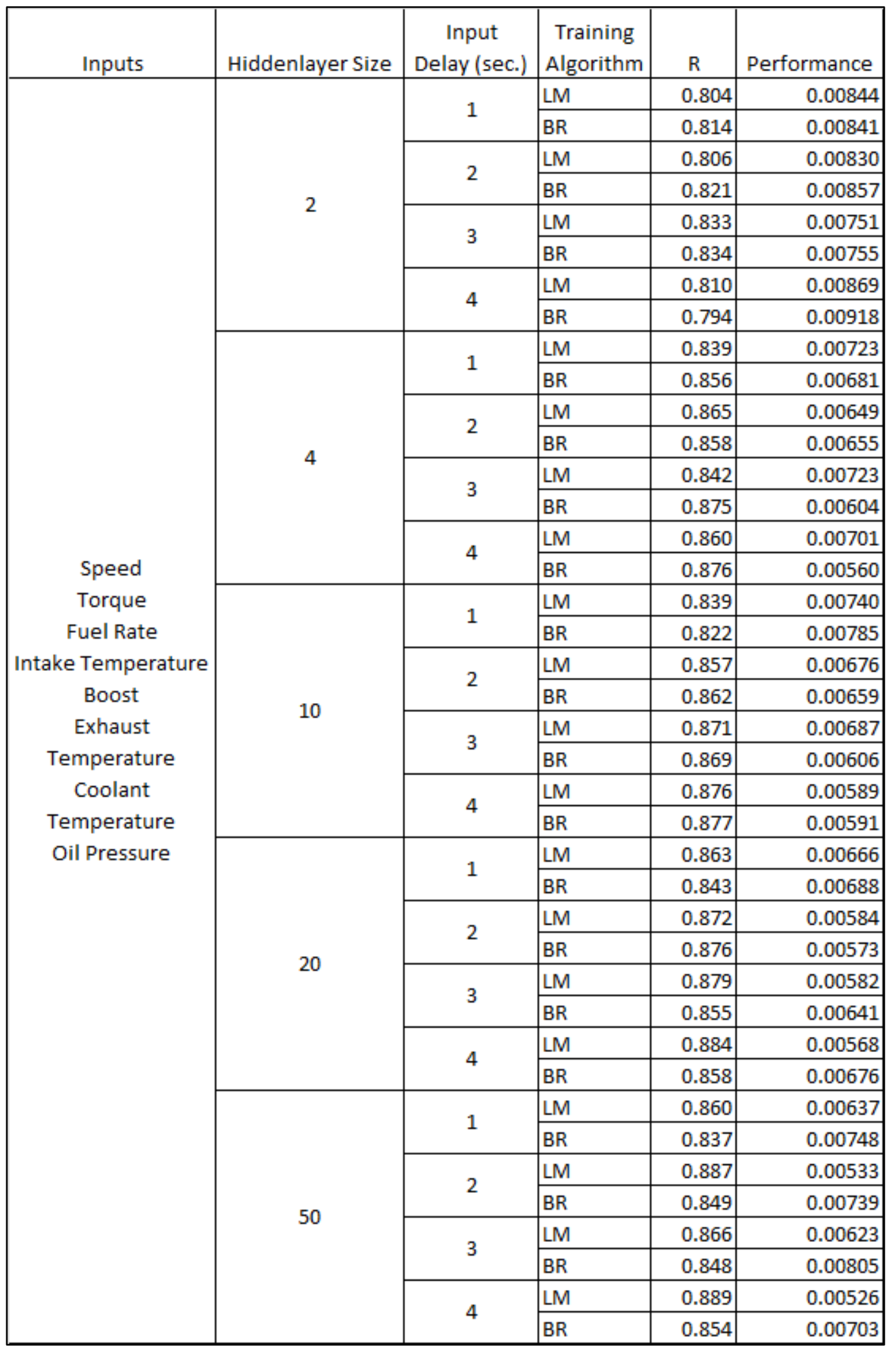


Table 7-Test Matrix with Input Set 2

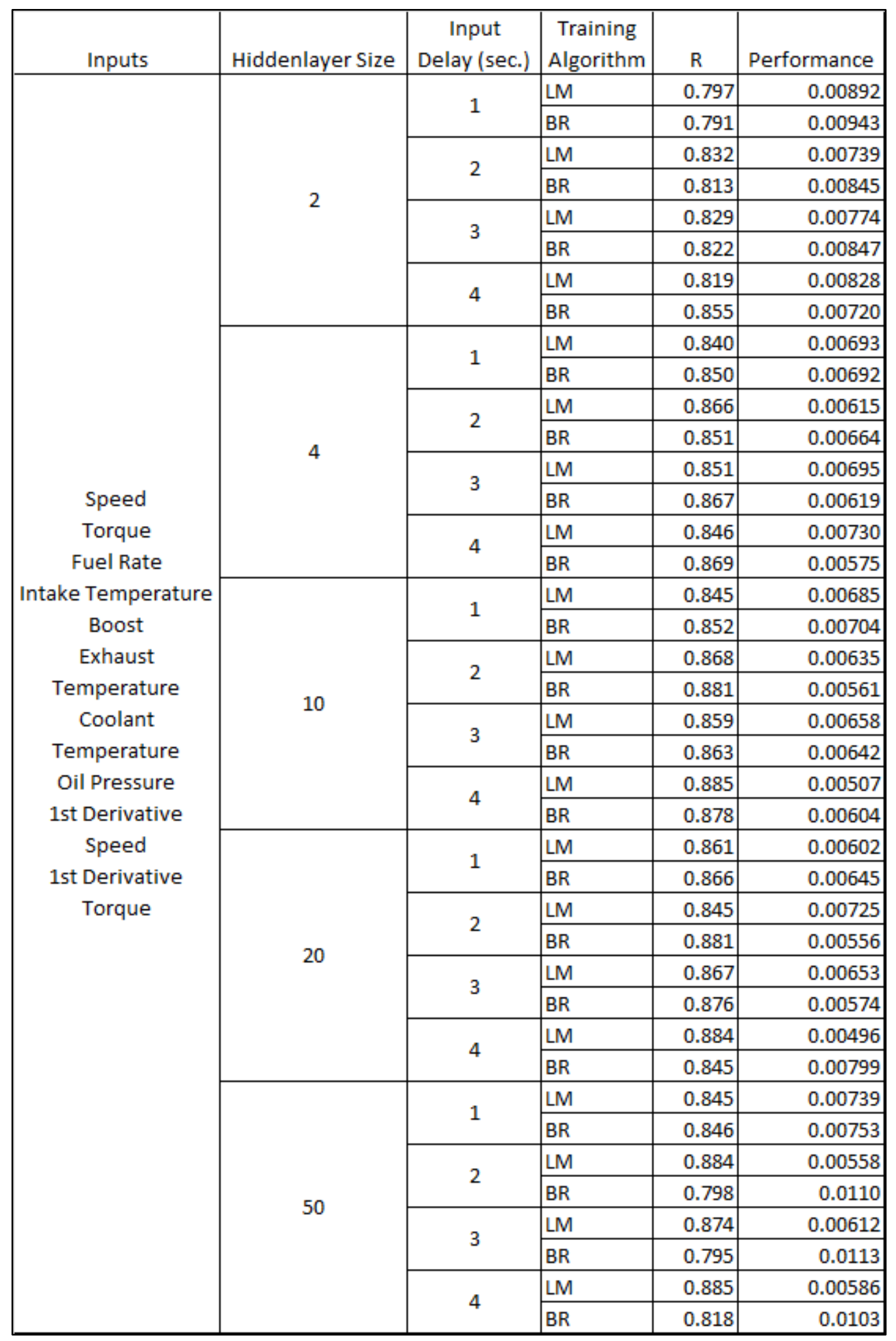


Table 8-Test Matrix with Input Set 3

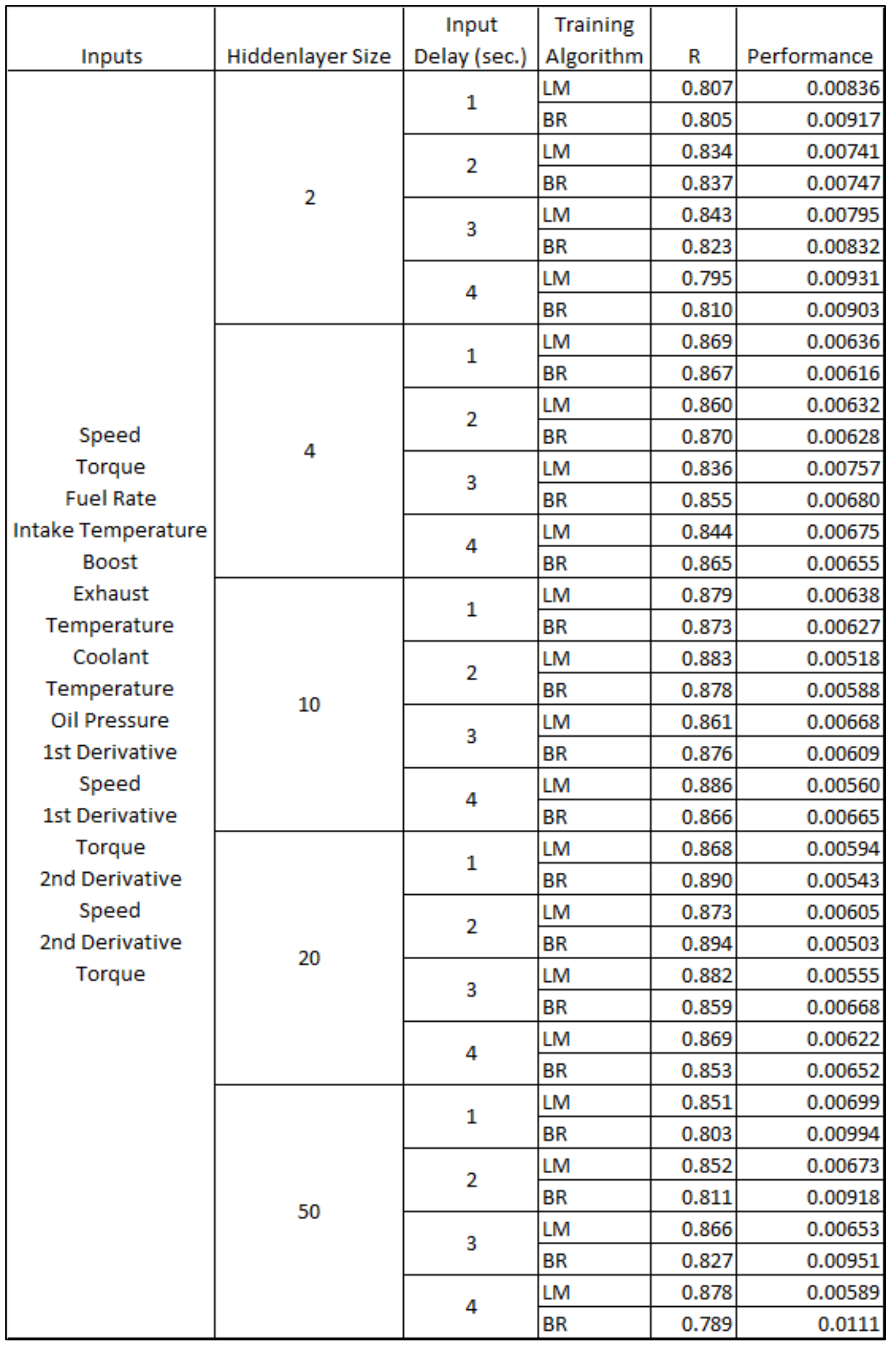


Table 9-Test Matrix with Input Set 4

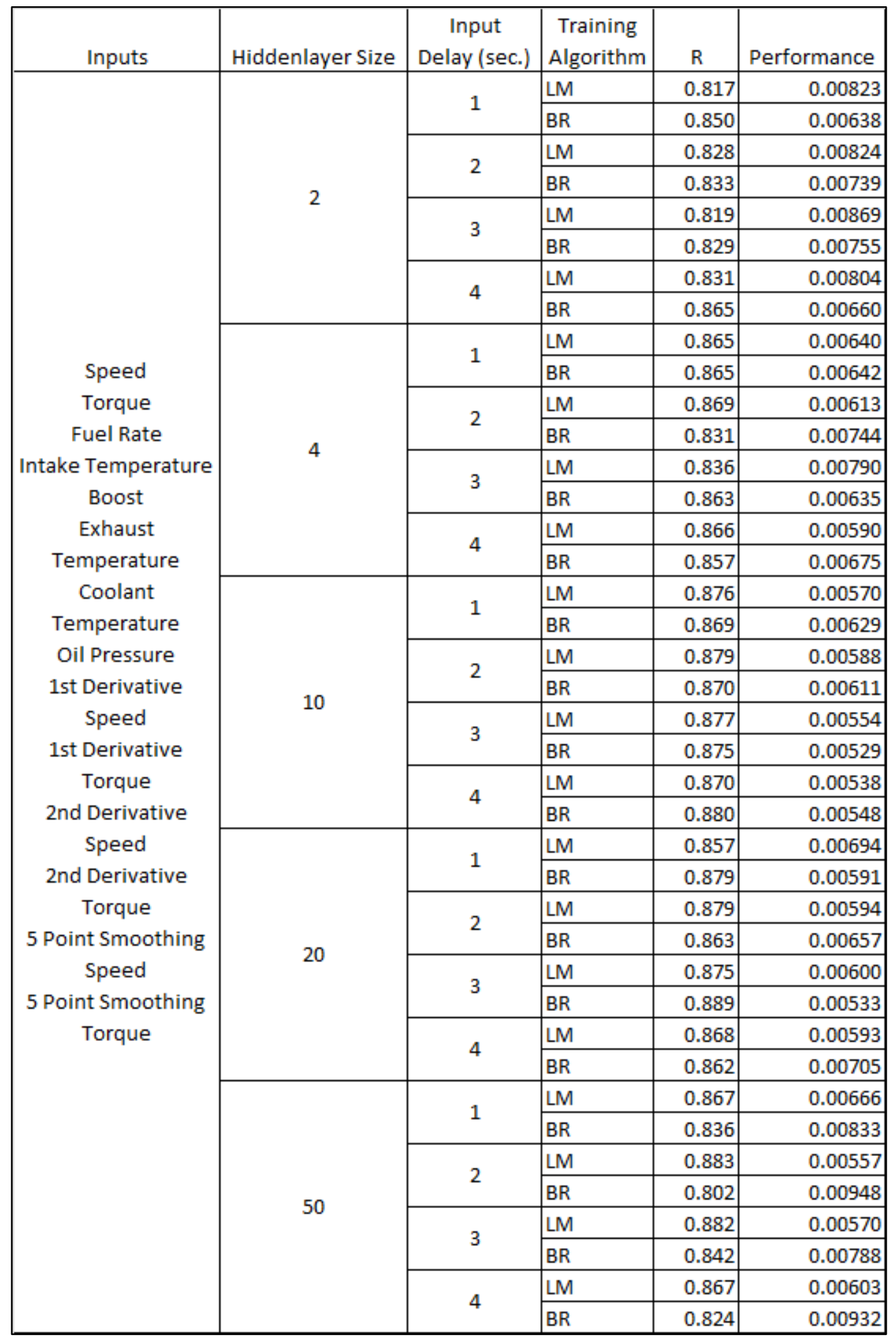


After obtaining the Performance and $\mathrm{R}$ value for each of the test combinations, the values could be plotted against each other. Figure 8 shows the scatter plot of performance versus $\mathrm{R}$ value for the preliminary model tests. A minimal performance value and a maximum $\mathrm{R}$ value is desired for the best prediction capabilities. Since the results were linear it was possible to determine which point corresponded to the optimal configuration.

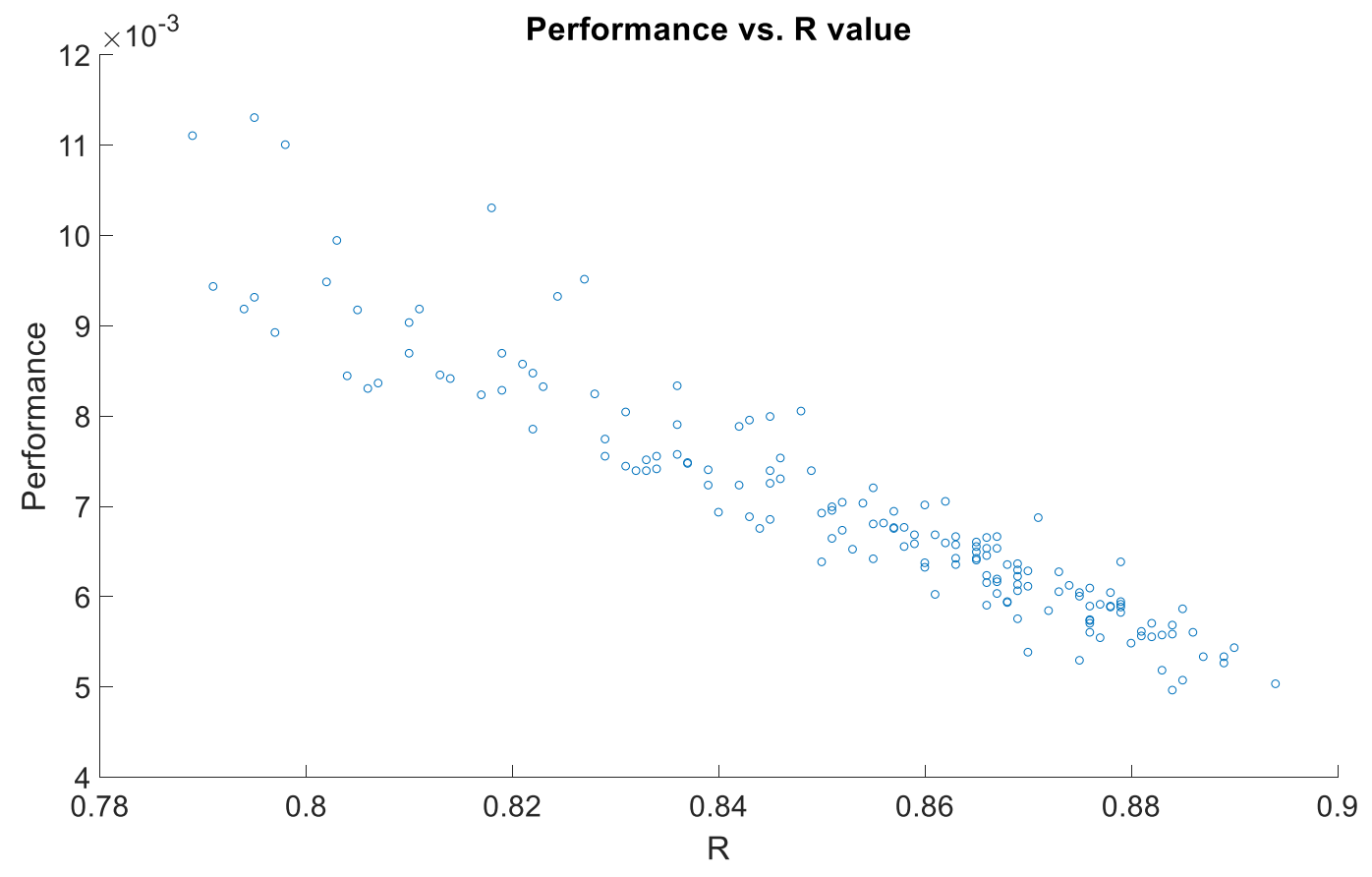

Figure 8-Performance vs. R Value

Once a model had been selected, it could be trained. Figure 9 shows the training output when using Highway 1 for the training data, and Figure 10 shows the output of the training data for Training data set 2 . 


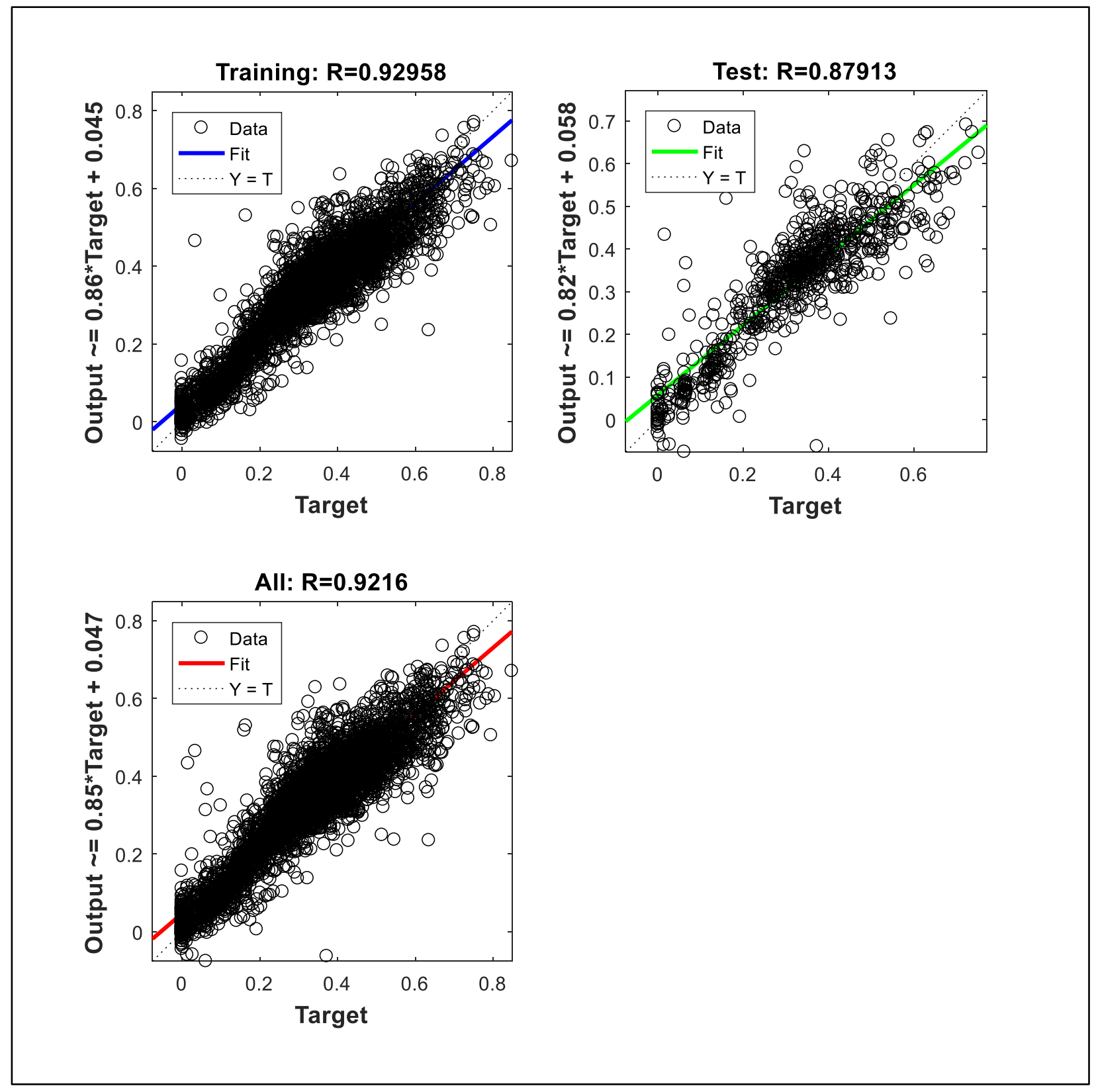

Figure 9-Training Output for Highway 1 


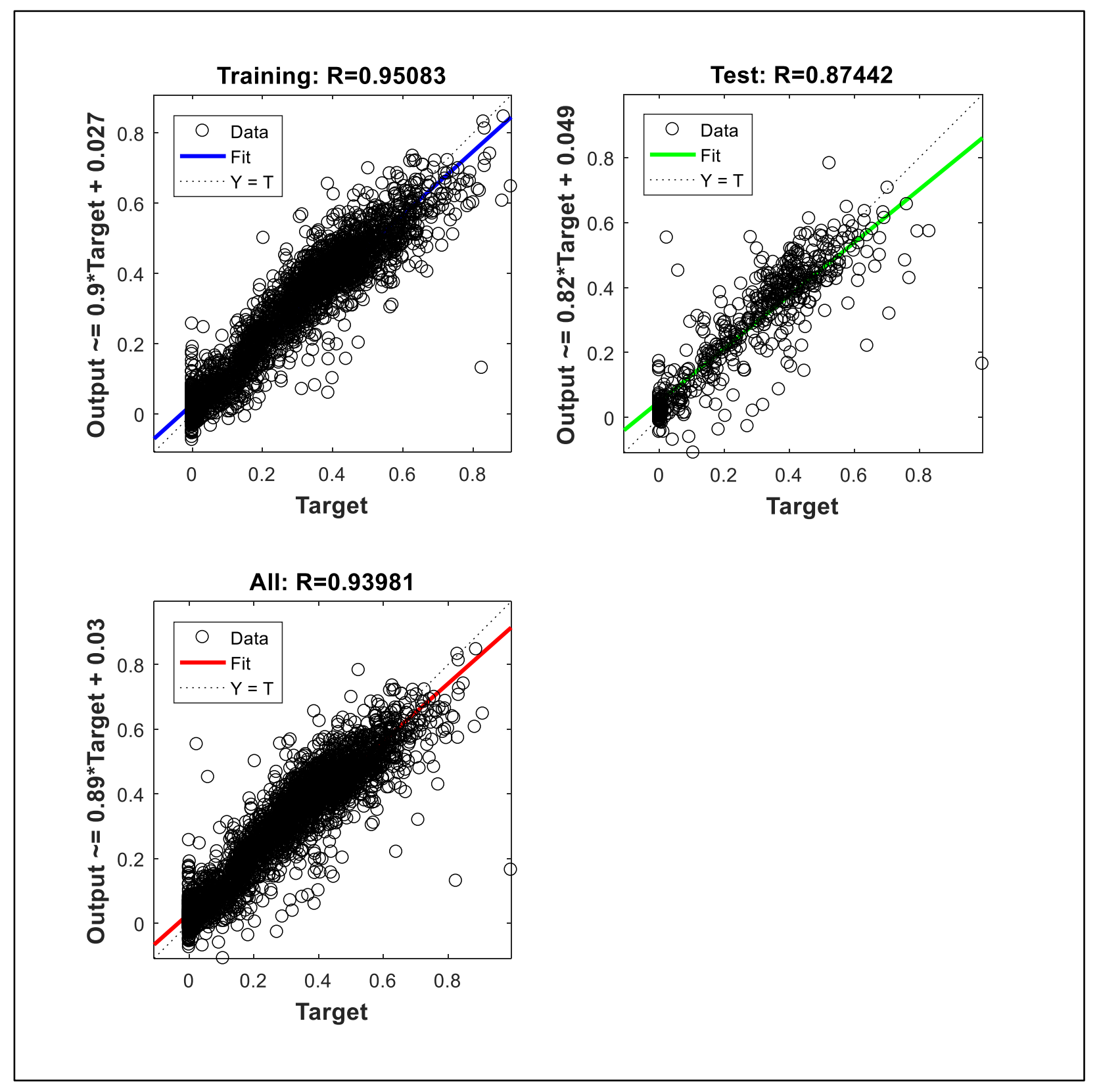

Figure 10-Training output for Combined Data

\subsection{TRAINING DATA SET 1}

In this section of the results, the validation tests of the neural network trained with training data set 1 will be shown. The percent error for each of the tests, a correlation plot, the five 
correlation values, and the errors in 200 second increments will be shown. The tables include the values for each of the five iterations and the average of these five values. The percent errors calculated are summed errors for the time under evaluation. For the total percent error, the actual and predicted values are summed over the length of the test and then compared. For the error in 200 seconds, the actual and predicted values are summed every 200 seconds and compared. The 200 second increment was arbitrarily chosen for evaluation in a shorter period compared to the length of the test data. The figures depicting the predicted NOx values versus the actual values over time can be seen in Appendix G.

\subsubsection{HIGHWAY 2}

The results of the validation testing of Highway 2 can be seen in Table 10 . The average error of the five validation tests was 5.37 percent.

Table 10-Highway 2 Validation Test Errors when Trained with Training Data 1

\begin{tabular}{|c|c|}
\hline \multicolumn{2}{|c|}{ Highway 2 } \\
\cline { 1 - 1 } Percent Error \\
\cline { 1 - 1 } Validation Iterations & Average \\
\cline { 1 - 1 } 5.52 & \multirow{2}{*}{5.37} \\
\cline { 1 - 1 } 5.02 & \\
\cline { 1 - 1 } 4.97 & \\
\hline 6.88 & \\
\cline { 1 - 1 } 4.44 &
\end{tabular}

To further describe the performance of the validation of the neural network with the Highway 2 test, the scatterplot of predicted versus actual sensor readings can be seen in Figure 11. This graph shows the correlation of predicted to actual values. The $R$ values for each of the five iterations can be seen in Table 11 . The average $R$ value was 0.79 which shows a strong correlation between the predicted and actual values. 


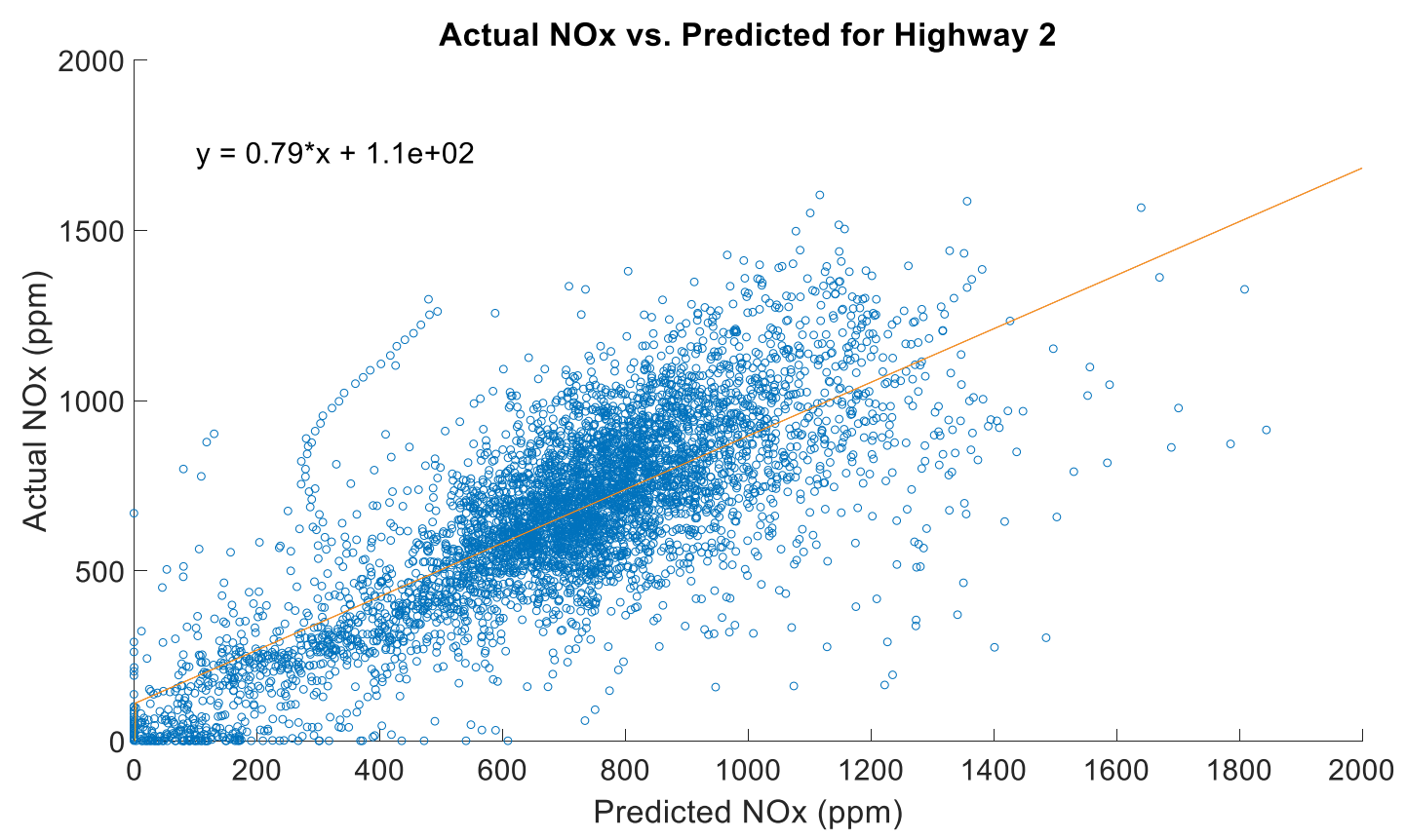

Figure 11-Scatterplot of Highway 2 Validation Test with NN Trained with Training Data 1

Table 11-R Values for Highway 2

\begin{tabular}{|c|c|}
\hline \multicolumn{2}{|c|}{ Highway 2} \\
\hline \multicolumn{2}{|c|}{ R Value } \\
\cline { 1 - 1 } Validation Iterations & Average \\
\cline { 1 - 1 } 0.79 & \multirow{2}{*}{0.79} \\
\cline { 1 - 1 } 0.81 & \\
\cline { 1 - 1 } 0.78 & \\
\cline { 1 - 1 } 0.80 & \\
\cline { 1 - 2 } 0.77 &
\end{tabular}

The last measure of performance was the evaluation of the error in 200 second segments along the test. These values can be seen in Table 12. In the 2001-2201 second range the error is much higher than anywhere else, due to uncharacteristically accelerations within this time period. With a few other exceptions, the error values are below ten percent for all five iterations. 
Table 12-Highway 2-200 Second Errors

\begin{tabular}{|l|r|r|r|r|r|}
\hline \multicolumn{7}{|c|}{ Highway 2 with Training Data Set 1 } \\
\hline & \multicolumn{1}{|c|}{ 200 Second Percent Errors } \\
\hline Time (s) & Test 1 & \multicolumn{1}{c|}{ Test 2 } & \multicolumn{1}{|c|}{ Test 3 } & \multicolumn{1}{c|}{ Test 4 } & \multicolumn{1}{c|}{ Test 5 } \\
\hline $1-201$ & 7.23 & 13.09 & 4.83 & 15.16 & 8.44 \\
\hline $201-401$ & 11.04 & 9.91 & 6.73 & 11.95 & 10.43 \\
\hline $401-601$ & 5.46 & 5.99 & 4.63 & 8.42 & 5.13 \\
\hline $601-801$ & 4.43 & 4.40 & 3.15 & 6.49 & 4.09 \\
\hline $801-1001$ & 7.76 & 8.67 & 10.39 & 10.04 & 7.15 \\
\hline $1001-1201$ & 2.82 & 3.26 & 4.76 & 2.72 & 1.47 \\
\hline $1201-1401$ & 4.64 & 4.05 & 5.77 & 5.88 & 2.94 \\
\hline $1401-1601$ & 6.08 & 5.88 & 8.55 & 5.12 & 3.39 \\
\hline $1601-1801$ & 10.44 & 11.82 & 9.27 & 9.27 & 6.23 \\
\hline $1801-2001$ & 5.04 & 5.89 & 7.04 & 0.52 & 10.41 \\
\hline $2001-2201$ & 20.04 & 22.23 & 23.03 & 27.73 & 19.01 \\
\hline $2201-2401$ & 0.93 & 2.29 & 3.57 & 4.34 & 3.14 \\
\hline $2401-2601$ & 1.89 & 1.52 & 1.78 & 5.96 & 4.06 \\
\hline $2601-2801$ & 2.42 & 2.68 & 1.76 & 0.43 & 1.51 \\
\hline $2801-3001$ & 1.34 & 2.92 & 3.85 & 4.90 & 2.46 \\
\hline $3001-3201$ & 10.13 & 11.31 & 12.17 & 12.12 & 10.18 \\
\hline $3201-3401$ & 5.82 & 5.54 & 7.41 & 8.74 & 5.04 \\
\hline $3401-3601$ & 1.70 & 1.39 & 2.56 & 4.46 & 1.98 \\
\hline $3601-3801$ & 3.07 & 3.35 & 2.87 & 6.88 & 3.12 \\
\hline $3801-4001$ & 1.38 & 3.31 & 3.20 & 8.55 & 5.77 \\
\hline $4001-4201$ & 6.26 & 5.70 & 5.87 & 7.91 & 4.77 \\
\hline $4201-4401$ & 8.66 & 6.19 & 5.93 & 8.22 & 7.47 \\
\hline $4401-4601$ & 3.20 & 4.21 & 4.92 & 2.10 & 3.76 \\
\hline $4601-4801$ & 13.64 & 10.48 & 10.59 & 13.89 & 14.85 \\
\hline $4801-5001$ & 6.12 & 8.40 & 8.54 & 8.50 & 7.45 \\
\hline
\end{tabular}

\subsubsection{HIGHWAY 3}

The Highway 3 validation test using training data set 1 had the best results, in terms of percent error, in this entire study. The resulting predicted versus actual sensor readings can be seen in Figure 12. To better observe how the prediction matches the actual values, an enlarged portion of the test is shown in Figure 13. 


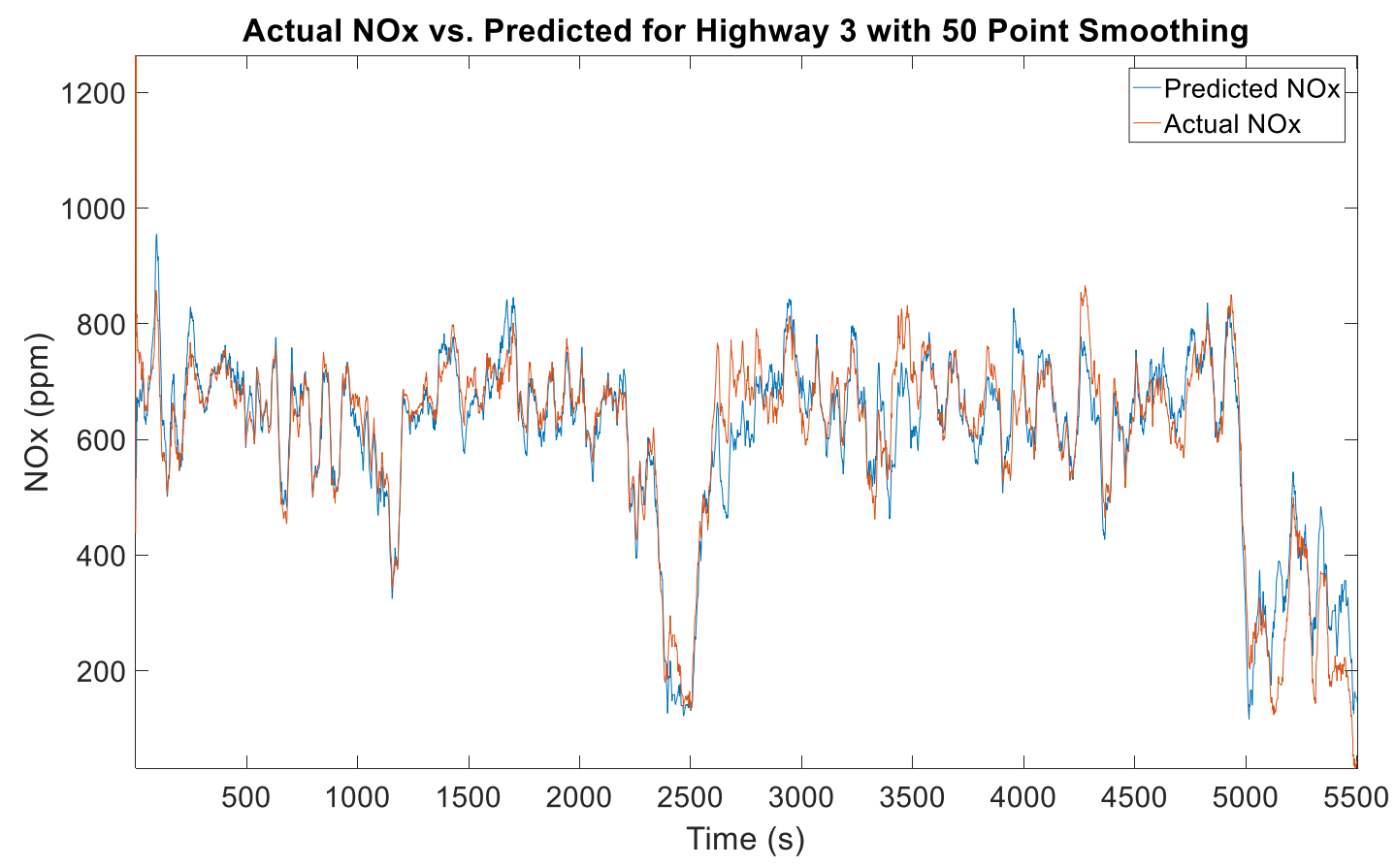

Figure 12-Actual vs. Predicted NOx for Highway 3 with 50 Point Smoothing

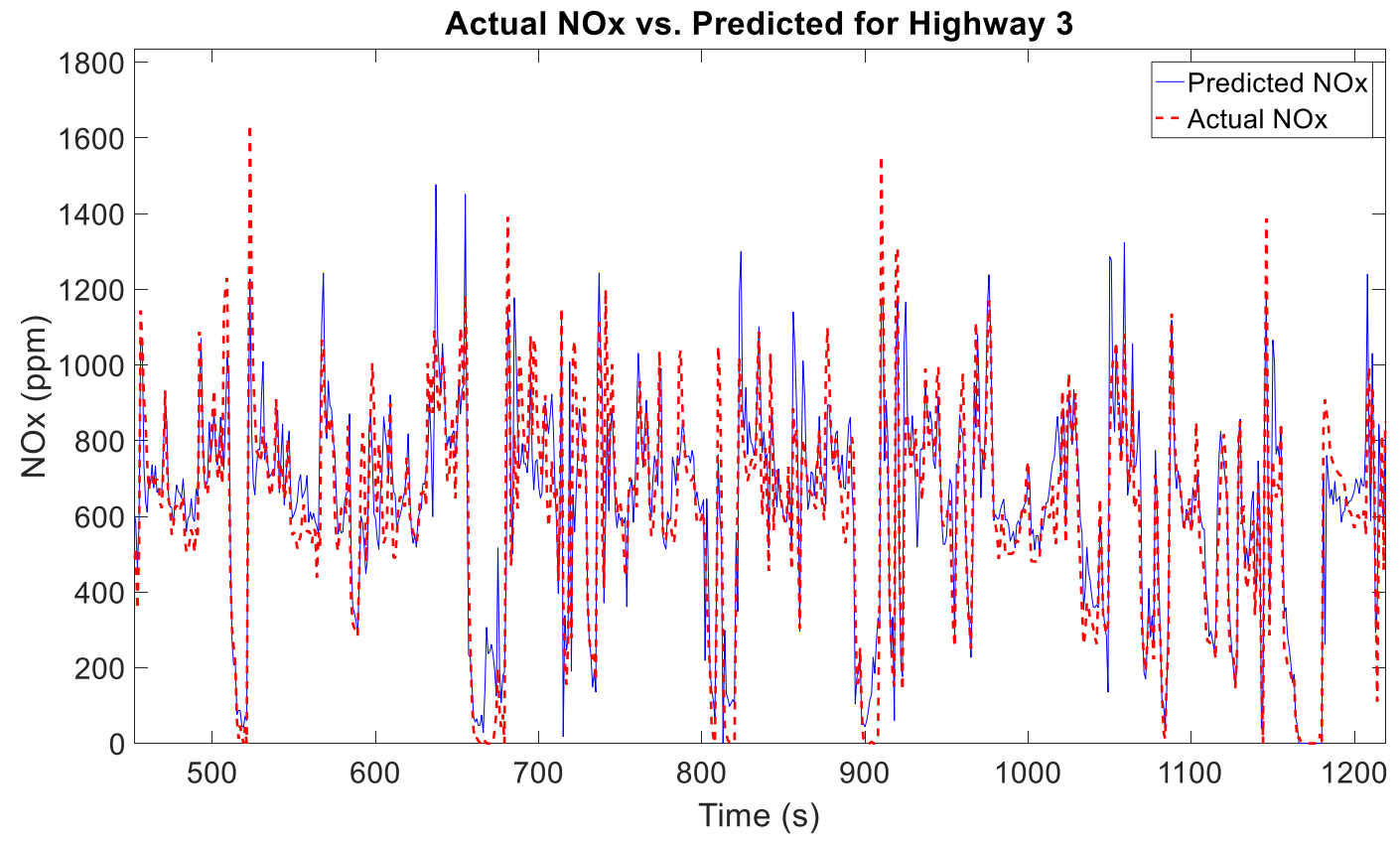

Figure 13-Enlarged Actual vs. Predicted for Highway 3

The percent error values for all five iterations can be seen in Table 13. All five of the tests resulted in less than two percent error over the length of the test. 
Table 13- Highway 3 Validation Test Errors when Trained with Training Data 1

\begin{tabular}{|c|c|}
\hline \multicolumn{2}{|c|}{ Highway 3} \\
\hline \multicolumn{2}{|c|}{ Percent Error } \\
\cline { 1 - 1 } Validation Iterations & Average \\
\hline 1.84 & \multirow{2}{*}{0.93} \\
\cline { 1 - 1 } 0.87 & \\
\cline { 1 - 1 } 0.00 & \\
\hline 0.56 & \\
\cline { 1 - 1 } 1.36 & \\
\hline
\end{tabular}

Figure 14 shows the correlation of predicted to actual values for Highway 3. The R values for each of the five iterations can be seen in Table 14 . The average $R$ value was 0.83 , which shows a strong correlation between the predicted and actual values.

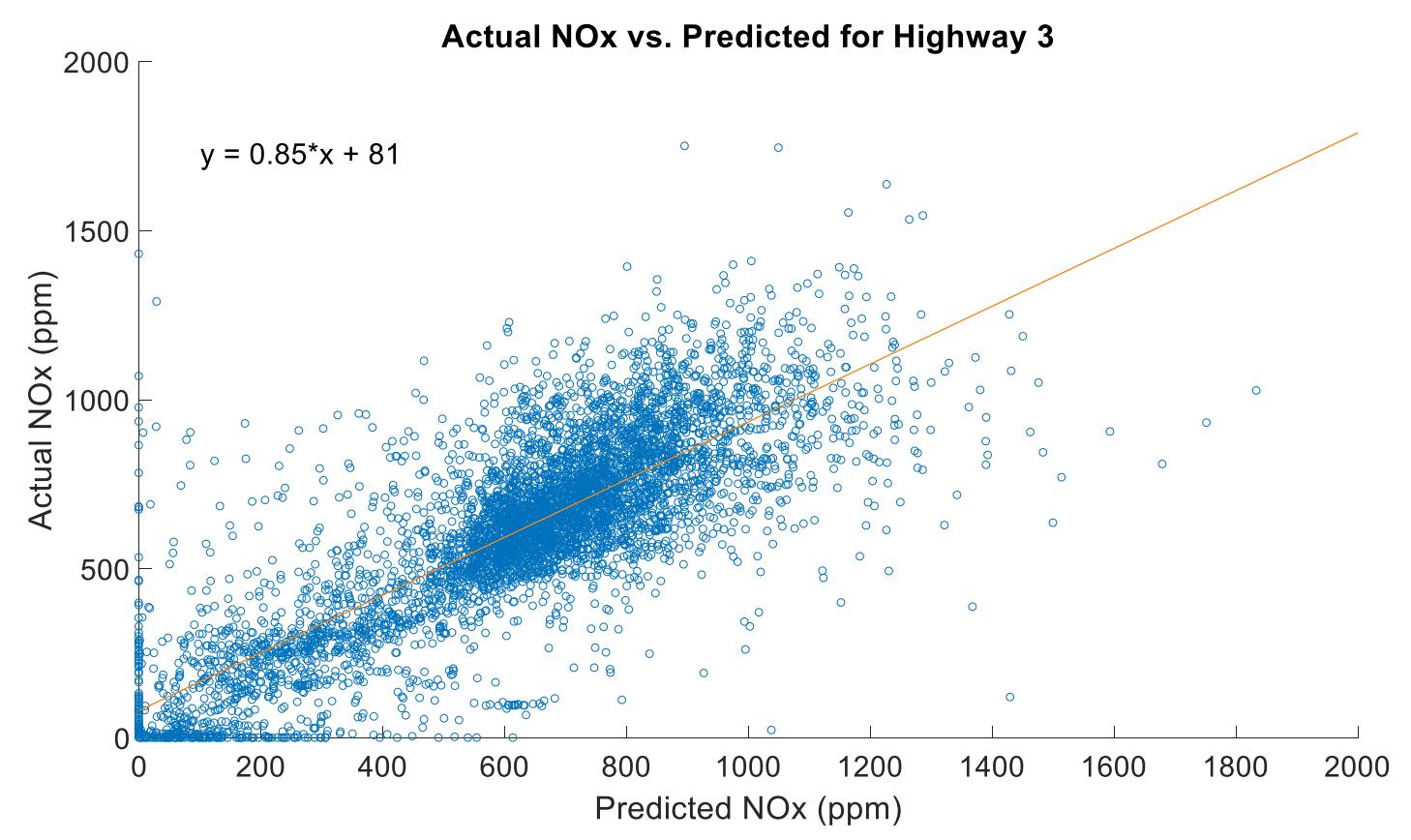

Figure 14- Scatterplot of Highway 3 Validation Test with NN Trained with Training Data 1 
Table 14-R Values for Highway 3

\begin{tabular}{|c|c|}
\hline \multicolumn{2}{|c|}{ Highway 3} \\
\cline { 1 - 1 } R Value \\
\cline { 1 - 1 } Validation Iterations & Average \\
\cline { 1 - 1 } 0.83 & \\
\cline { 1 - 1 } 0.84 & \\
\cline { 1 - 1 } 0.84 & \\
\hline 0.84 & \\
\cline { 1 - 1 } 0.82 & \\
\hline
\end{tabular}

Table 15 shows the errors in 200 second increments for the Highway 3 test. In the 2500-2800 and 3400-3600 sections the error is due to uncharacteristic accelerations within these time periods. Excluding these two sections the errors are generally quite low, with almost all sections falling below five percent error. 
Table 15-Highway 3-200 Second Errors

\begin{tabular}{|l|r|r|r|r|r|}
\hline \multicolumn{7}{|c|}{ Highway 3 with Training Data Set 1 } \\
\hline & \multicolumn{1}{|c|}{ 200 Second Percent Errors } \\
\hline Time (s) & Test 1 & \multicolumn{1}{|c|}{ Test 2 } & \multicolumn{1}{c|}{ Test 3 } & \multicolumn{1}{|c|}{ Test 4 } & \multicolumn{1}{c|}{ Test 5 } \\
\hline $1-201$ & 2.39 & 1.15 & 4.18 & 4.85 & 4.12 \\
\hline $201-401$ & 2.35 & 0.79 & 1.28 & 2.20 & 1.99 \\
\hline $401-601$ & 2.23 & 0.64 & 1.33 & 1.24 & 2.36 \\
\hline $601-801$ & 2.16 & 0.87 & 1.95 & 1.90 & 2.78 \\
\hline $801-1001$ & 1.42 & 2.02 & 0.98 & 2.55 & 0.89 \\
\hline $1001-1201$ & 4.08 & 5.24 & 5.30 & 3.83 & 4.52 \\
\hline $1201-1401$ & 0.36 & 0.57 & 0.25 & 0.13 & 0.44 \\
\hline $1401-1601$ & 4.62 & 4.00 & 5.46 & 4.75 & 3.56 \\
\hline $1601-1801$ & 2.04 & 1.82 & 1.87 & 1.94 & 2.58 \\
\hline $1801-2001$ & 2.51 & 3.35 & 3.52 & 2.19 & 1.30 \\
\hline $2001-2201$ & 0.06 & 0.41 & 1.28 & 0.63 & 0.66 \\
\hline $2201-2401$ & 0.04 & 4.41 & 2.53 & 1.30 & 2.85 \\
\hline $2401-2601$ & 11.28 & 22.44 & 5.19 & 2.32 & 1.78 \\
\hline $2601-2801$ & 16.45 & 15.41 & 15.88 & 15.85 & 15.13 \\
\hline $2801-3001$ & 3.87 & 4.41 & 3.29 & 4.54 & 4.13 \\
\hline $3001-3201$ & 0.42 & 1.31 & 2.74 & 1.65 & 0.70 \\
\hline $3201-3401$ & 3.55 & 3.70 & 3.03 & 5.21 & 2.55 \\
\hline $3401-3601$ & 10.09 & 9.63 & 9.95 & 9.72 & 7.34 \\
\hline $3601-3801$ & 1.26 & 0.88 & 2.70 & 0.85 & 2.00 \\
\hline $3801-4001$ & 3.21 & 4.10 & 2.64 & 2.18 & 2.43 \\
\hline $4001-4201$ & 1.39 & 1.80 & 0.60 & 0.24 & 1.46 \\
\hline $4201-4401$ & 7.22 & 7.13 & 7.86 & 7.97 & 8.65 \\
\hline $4401-4601$ & 0.75 & 2.01 & 0.47 & 1.97 & 1.30 \\
\hline $4601-4801$ & 5.38 & 5.06 & 4.27 & 6.29 & 3.30 \\
\hline $4801-5001$ & 1.82 & 0.25 & 1.82 & 0.19 & 0.72 \\
\hline
\end{tabular}

\subsubsection{REGIONAL}

Table 16 shows the percent errors for each of the five iterations for the Regional tests. The results for this test were inconsistent with errors ranging between 1.08 and 26.3 percent. 
Table 16-Regional Validation Test Errors when Trained with Training Data 1

\begin{tabular}{|c|c|}
\hline \multicolumn{2}{|c|}{ Regional } \\
\cline { 1 - 1 } Percent Error \\
\cline { 1 - 1 } Validation Iterations & \multirow{2}{*}{ Average } \\
\cline { 1 - 1 } 1.08 & \\
\cline { 1 - 1 } 11.12 & \\
\cline { 1 - 1 } 3.93 & \\
\hline 13.84 & \\
\cline { 1 - 1 } 26.30 & \\
\hline
\end{tabular}

It can be seen is Figure 15 that there is correlation between the predicted and actual values, but the spread of points is much greater than the Highway tests. Table 17 shows the correlation values for all five iterations.

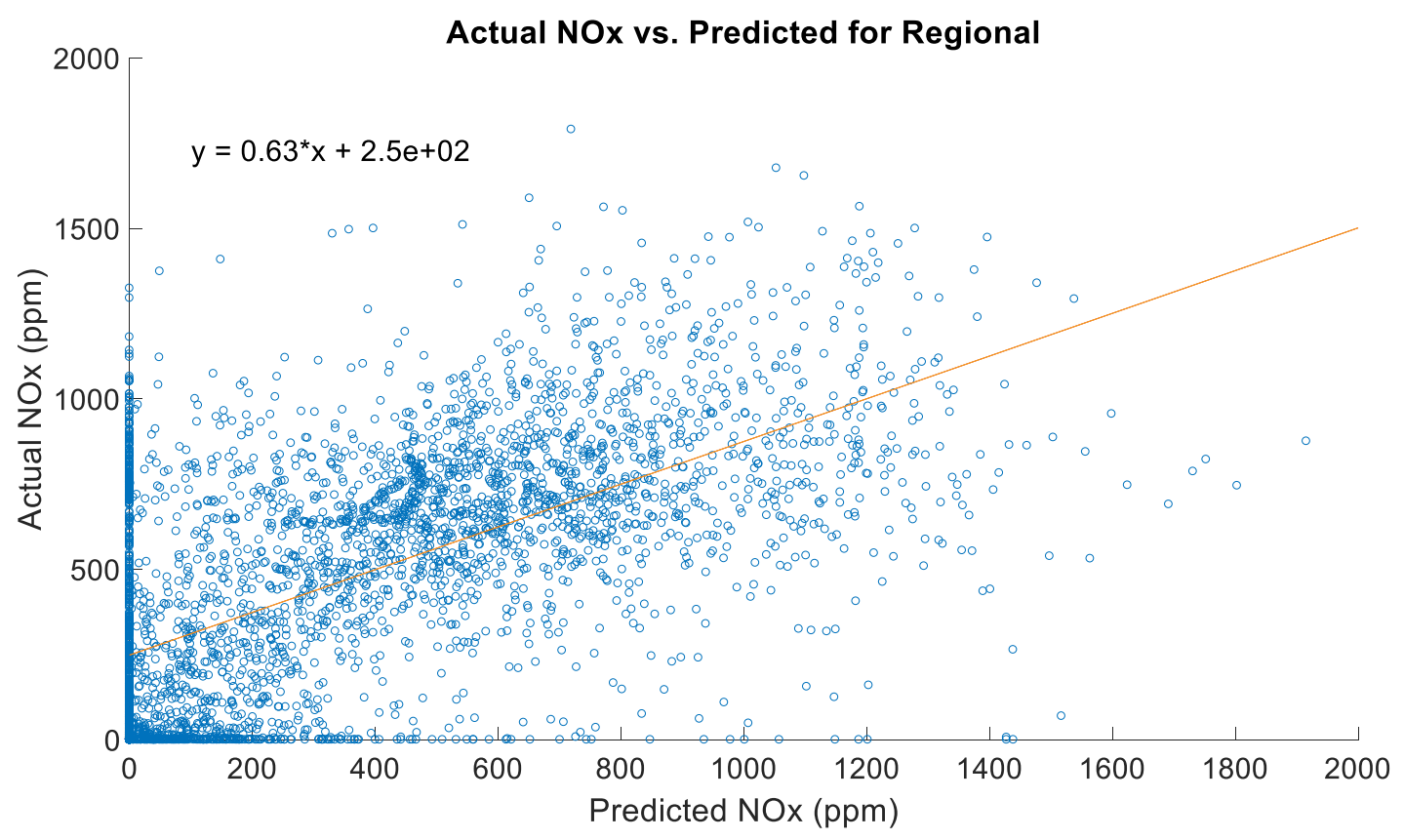

Figure 15- Scatterplot of Regional Validation Test with NN Trained with Training Data 1

Table 17-Regional R Values

\begin{tabular}{|c|c|}
\hline \multicolumn{2}{|c|}{ Regional } \\
\hline \multicolumn{2}{|c|}{ R Value } \\
\cline { 1 - 1 } Validation Iterations & Average \\
\hline 0.74 & \multirow{2}{*}{0.69} \\
\cline { 1 - 1 } 0.63 & \\
\cline { 1 - 1 } 0.73 & \\
\hline 0.71 & \\
\hline 0.63 & \\
\hline
\end{tabular}


In Table 18, the inconsistency of the prediction is evident. The error percentages range between 69.94 and 0.00 throughout all parts of the test.

Table 18-Regional-200 Second Errors

\begin{tabular}{|l|r|r|r|r|r|}
\hline \multicolumn{7}{|c|}{ Regional with Training Data Set 1 } \\
\hline & \multicolumn{2}{|c|}{ 200 Second Percent Errors } \\
\hline Time (s) & Test 1 & \multicolumn{1}{|c|}{ Test 2 } & \multicolumn{1}{|c|}{ Test 3 } & \multicolumn{1}{l|}{ Test 4 } & \multicolumn{1}{c|}{ Test 5 } \\
\hline $1-201$ & 3.44 & 8.25 & 1.58 & 13.68 & 3.20 \\
\hline $201-401$ & 17.51 & 21.84 & 36.36 & 27.64 & 20.32 \\
\hline $401-601$ & 7.32 & 0.78 & 25.15 & 9.37 & 8.37 \\
\hline $601-801$ & 45.39 & 51.30 & 69.94 & 46.47 & 87.59 \\
\hline $801-1001$ & 4.86 & 3.18 & 23.84 & 14.89 & 11.56 \\
\hline $1001-1201$ & 6.54 & 0.99 & 19.04 & 8.31 & 22.07 \\
\hline $1201-1401$ & 8.85 & 4.76 & 10.87 & 4.12 & 22.73 \\
\hline $1401-1601$ & 7.16 & 2.32 & 2.80 & 4.75 & 5.73 \\
\hline $1601-1801$ & 19.38 & 11.97 & 18.25 & 7.38 & 33.00 \\
\hline $1801-2001$ & 4.04 & 4.59 & 0.75 & 3.68 & 1.14 \\
\hline $2001-2201$ & 20.89 & 9.32 & 1.69 & 17.21 & 18.64 \\
\hline $2201-2401$ & 8.67 & 11.22 & 13.35 & 4.85 & 23.88 \\
\hline $2401-2601$ & 22.77 & 3.72 & 84.04 & 60.97 & 27.65 \\
\hline $2601-2801$ & 17.98 & 18.05 & 2.30 & 0.00 & 17.61 \\
\hline $2801-3001$ & 12.42 & 11.20 & 10.98 & 6.48 & 1.46 \\
\hline $3001-3201$ & 13.70 & 14.73 & 15.00 & 13.47 & 4.52 \\
\hline $3201-3401$ & 12.82 & 13.10 & 17.86 & 11.68 & 5.91 \\
\hline $3401-3601$ & 0.79 & 3.80 & 3.32 & 2.98 & 9.91 \\
\hline & & & & & \\
\hline
\end{tabular}

\subsubsection{LOCAL}

The Local validation test resulted in the worst prediction of any test performed, in terms of percent error. The percent error was as high as 70.75 percent for one of the iterations. The error percentages for all the iterations can be seen in Table 19. 
Table 19- Local Validation Test Errors when Trained with Training Data 1

\begin{tabular}{|c|c|}
\hline \multicolumn{2}{|c|}{ Local } \\
\cline { 1 - 1 } Percent Error \\
\cline { 1 - 1 } Validation Iterations & \multirow{3}{*}{ Average } \\
\cline { 1 - 1 } 70.75 & \\
\cline { 1 - 1 } 15.39 & \\
\cline { 1 - 1 } 11.42 & \\
\cline { 1 - 1 } 33.17 & \\
\cline { 1 - 1 } 33.74 & \\
\hline
\end{tabular}

Figure 16 shows the correlation between actual and predicted values of the Local test.

The correlation values for all the iterations can be seen in Table 20. Although the Local test was the worst performance of the neural network, the average $\mathrm{R}$ value was still a 0.6 , which means there is still correlation between the actual and predicted values.

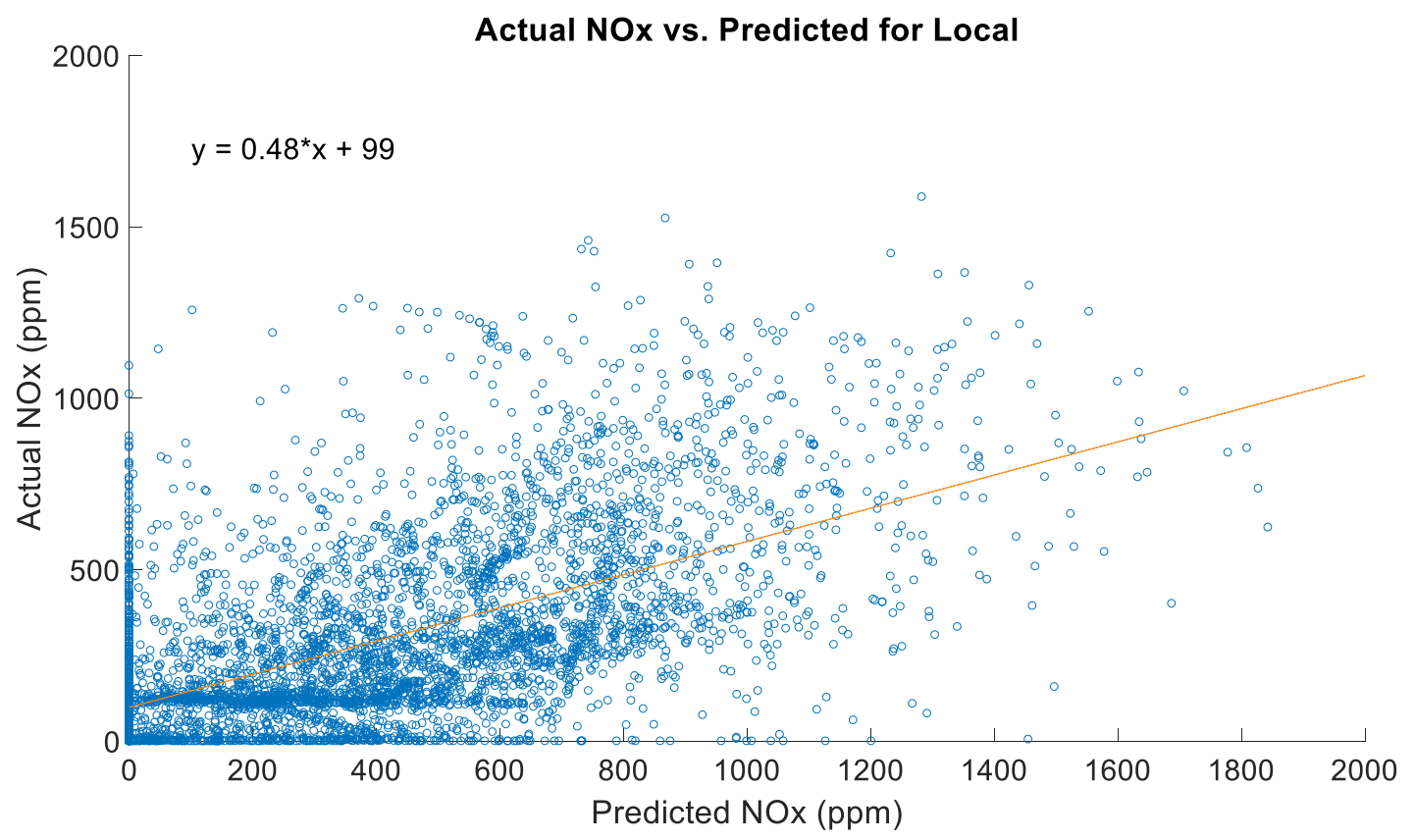

Figure 16- Scatterplot of Local Validation Test with NN Trained with Training Data 1 
Table 20-Local R Values

\begin{tabular}{|c|c|}
\hline \multicolumn{2}{|c|}{ Local } \\
\cline { 1 - 1 } R Value \\
\cline { 1 - 1 } Validation Iterations & \multirow{2}{*}{ Average } \\
\cline { 1 - 1 } 0.44 & \\
\cline { 1 - 1 } 0.70 & \\
\cline { 1 - 1 } 0.64 & \\
\cline { 1 - 1 } 0.64 & \\
\cline { 1 - 1 } 0.58 & \\
\cline { 1 - 1 } &
\end{tabular}

Table 21 shows how poorly the neural network predicted for the Local test. Errors as high as 155.64 percent can be seen.

Table 21-Local-200 Second Errors

\begin{tabular}{|l|r|r|r|r|r|}
\hline \multicolumn{7}{|c|}{ Local with Training Data Set 1 } \\
\hline & \multicolumn{1}{|c|}{ 200 Second Percent Errors } \\
\hline Time (s) & \multicolumn{1}{|c|}{ Test 1 } & \multicolumn{1}{|c|}{ Test 2 } & \multicolumn{1}{|c|}{ Test 3 } & \multicolumn{1}{c|}{ Test 4 } & \multicolumn{1}{c|}{ Test 5 } \\
\hline $1-201$ & 7.84 & 1.64 & 3.06 & 0.66 & 17.22 \\
\hline $201-401$ & 14.05 & 6.50 & 0.29 & 6.51 & 7.37 \\
\hline $401-601$ & 29.51 & 11.57 & 5.50 & 8.94 & 27.40 \\
\hline $601-801$ & 17.28 & 8.12 & 23.66 & 14.75 & 8.82 \\
\hline $801-1001$ & 4.14 & 6.08 & 0.75 & 14.97 & 1.91 \\
\hline $1001-1201$ & 0.82 & 4.12 & 8.34 & 3.26 & 16.33 \\
\hline $1201-1401$ & 21.86 & 33.13 & 25.47 & 30.68 & 8.23 \\
\hline $1401-1601$ & 83.79 & 86.75 & 2.61 & 34.13 & 27.06 \\
\hline $1601-1801$ & 109.23 & 96.18 & 35.42 & 112.95 & 44.01 \\
\hline $1801-2001$ & 86.35 & 97.87 & 53.78 & 12.89 & 31.47 \\
\hline $2001-2201$ & 59.43 & 68.35 & 40.52 & 0.50 & 49.63 \\
\hline $2201-2401$ & 78.79 & 86.39 & 45.79 & 7.86 & 45.00 \\
\hline $2401-2601$ & 105.05 & 99.05 & 42.84 & 1.69 & 60.46 \\
\hline $2601-2801$ & 72.35 & 79.35 & 37.80 & 5.99 & 39.75 \\
\hline $2801-3001$ & 105.54 & 108.49 & 7.26 & 46.70 & 37.40 \\
\hline $3001-3201$ & 155.64 & 134.32 & 43.21 & 110.90 & 115.21 \\
\hline $3201-3401$ & 123.62 & 100.39 & 77.18 & 62.18 & 72.00 \\
\hline $3401-3601$ & 70.60 & 72.93 & 12.57 & 20.49 & 53.67 \\
\hline $3601-3801$ & 97.98 & 85.69 & 8.19 & 31.18 & 72.02 \\
\hline $3801-4001$ & 115.90 & 120.41 & 13.46 & 2.19 & 93.47 \\
\hline $4001-4201$ & 89.64 & 95.70 & 14.36 & 49.33 & 22.53 \\
\hline $4201-4401$ & 91.25 & 101.05 & 40.16 & 65.74 & 29.85 \\
\hline
\end{tabular}




\subsubsection{NEARDOCK}

The results for the Neardock test show average prediction for this location. The errors for each of the iterations can be seen in Table 22. The errors are higher than the Highway tests but less than the Regional and Interstate tests.

Table 22- Neardock Validation Test Errors when Trained with Training Data 1

\begin{tabular}{|c|c|}
\hline \multicolumn{2}{|c|}{ Neardock } \\
\hline \multicolumn{2}{|c|}{ Percent Error } \\
\cline { 1 - 1 } Validation Iterations & Average \\
\hline 21.60 & \multirow{2}{*}{19.67} \\
\cline { 1 - 1 } 14.78 & \\
\hline 10.35 & \\
\cline { 1 - 1 } 14.20 & \\
\hline 37.41 &
\end{tabular}

Figure 17 shows the correlation of predicted to actual values. The correlation values can be seen in Table 23. The average correlation value is 0.63 .

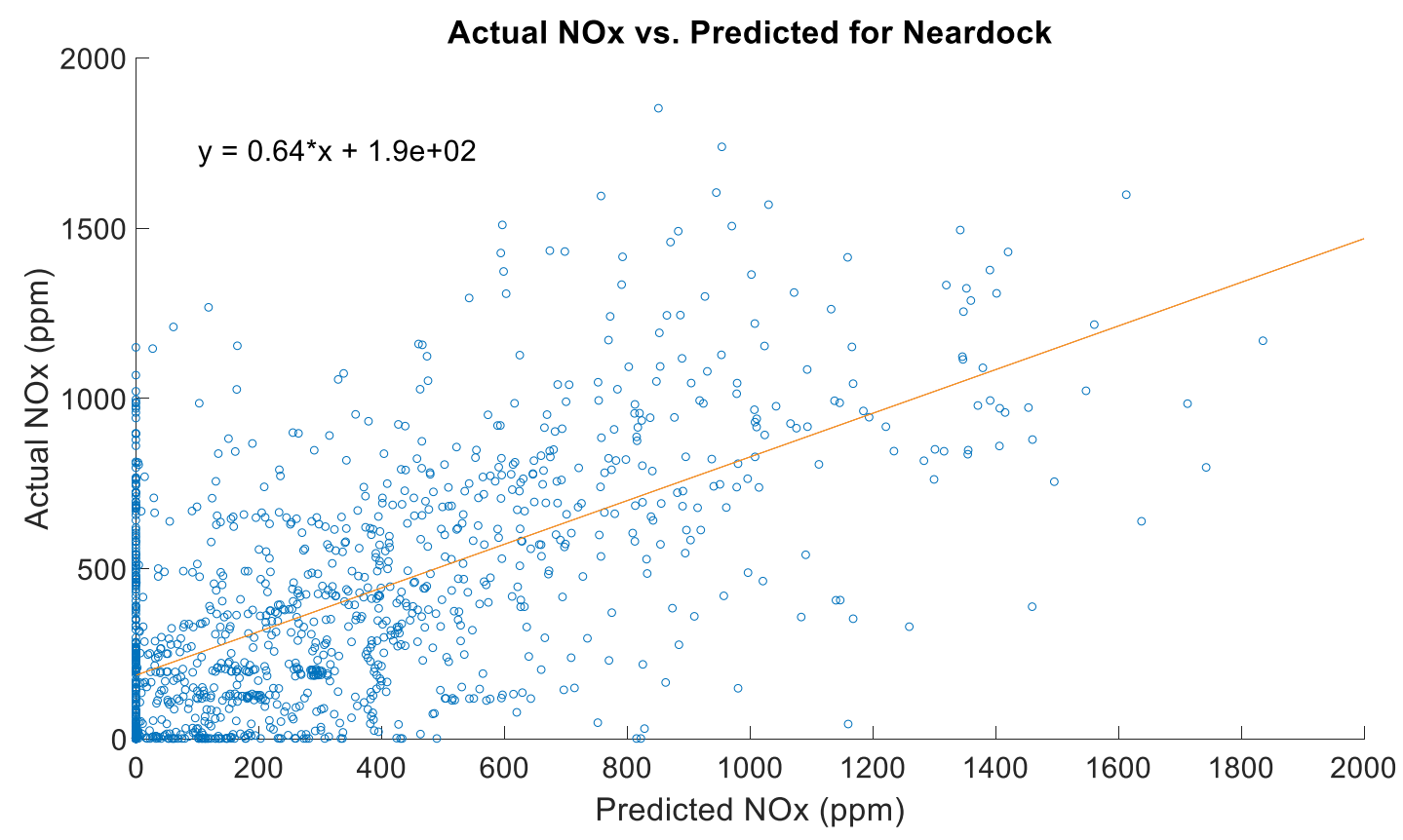

Figure 17- Scatterplot of Neardock Validation Test with NN Trained with Training Data 1 
Table 23-Neardock $R$ Values

\begin{tabular}{|c|c|}
\hline \multicolumn{2}{|c|}{ Neardock } \\
\cline { 1 - 1 } R Value \\
\cline { 1 - 1 } Validation Iterations & \multirow{2}{*}{ Average } \\
\cline { 1 - 1 } 0.65 & \\
\cline { 1 - 1 } 0.65 & \\
\cline { 1 - 1 } 0.67 & \\
\cline { 1 - 1 } 0.57 & \\
\cline { 1 - 1 } 0.63 & \\
\hline
\end{tabular}

Table 24 shows the errors in 200 second increments. Since the Neardock data set is much shorter than the rest of the tests, there are less results to compare.

Table 24-Neardock Percent Errors

\begin{tabular}{|l|r|r|r|r|r|}
\hline \multicolumn{7}{|c|}{ Neardock with Training Data Set 1 } \\
\hline & \multicolumn{5}{|c|}{ 200 Second Percent Errors } \\
\hline Time (s) & Test 1 & Test 2 & \multicolumn{1}{c|}{ Test 3 } & Test 4 & Test 5 \\
\hline $1-201$ & 13.18 & 14.78 & 9.71 & 25.02 & 6.75 \\
\hline $201-401$ & 19.53 & 8.72 & 11.31 & 8.22 & 25.06 \\
\hline $401-601$ & 45.69 & 43.16 & 39.02 & 54.50 & 75.30 \\
\hline $601-801$ & 22.04 & 45.93 & 37.17 & 42.42 & 67.78 \\
\hline $801-1001$ & 6.62 & 38.81 & 18.70 & 54.55 & 32.92 \\
\hline $1001-1201$ & 28.88 & 42.46 & 20.57 & 7.19 & 0.71 \\
\hline
\end{tabular}

\subsubsection{INTERSTATE 1}

The Interstate 1 validation test prodcued some interesting results. The average error in the five iterations was 24.29 percent, which can be seen in Table 25 . This error value does not describe an entirely accurate prediction, but the average correlation factor between the predicted and actual values is very strong at 0.8 , which can be seen in Table 26 . Figure 18 shows that while there is a strong correlation between the prediction and actual values, the spread of points ranges much further than the more accurate predictions of the Highway tests. 
Table 25-Interstate 1 Validation Test Errors when Trained with Training Data 1

\begin{tabular}{|c|c|}
\hline \multicolumn{2}{|c|}{ Interstate 1 } \\
\cline { 1 - 1 } Percent Error \\
\cline { 1 - 1 } Validation Iterations & \multirow{2}{*}{ Average } \\
\cline { 1 - 1 } 18.33 & \\
\cline { 1 - 1 } 23.65 & \\
\cline { 1 - 1 } 26.14 & \\
\cline { 1 - 1 } 20.13 & \\
\cline { 1 - 1 } 33.22 & \\
\hline
\end{tabular}

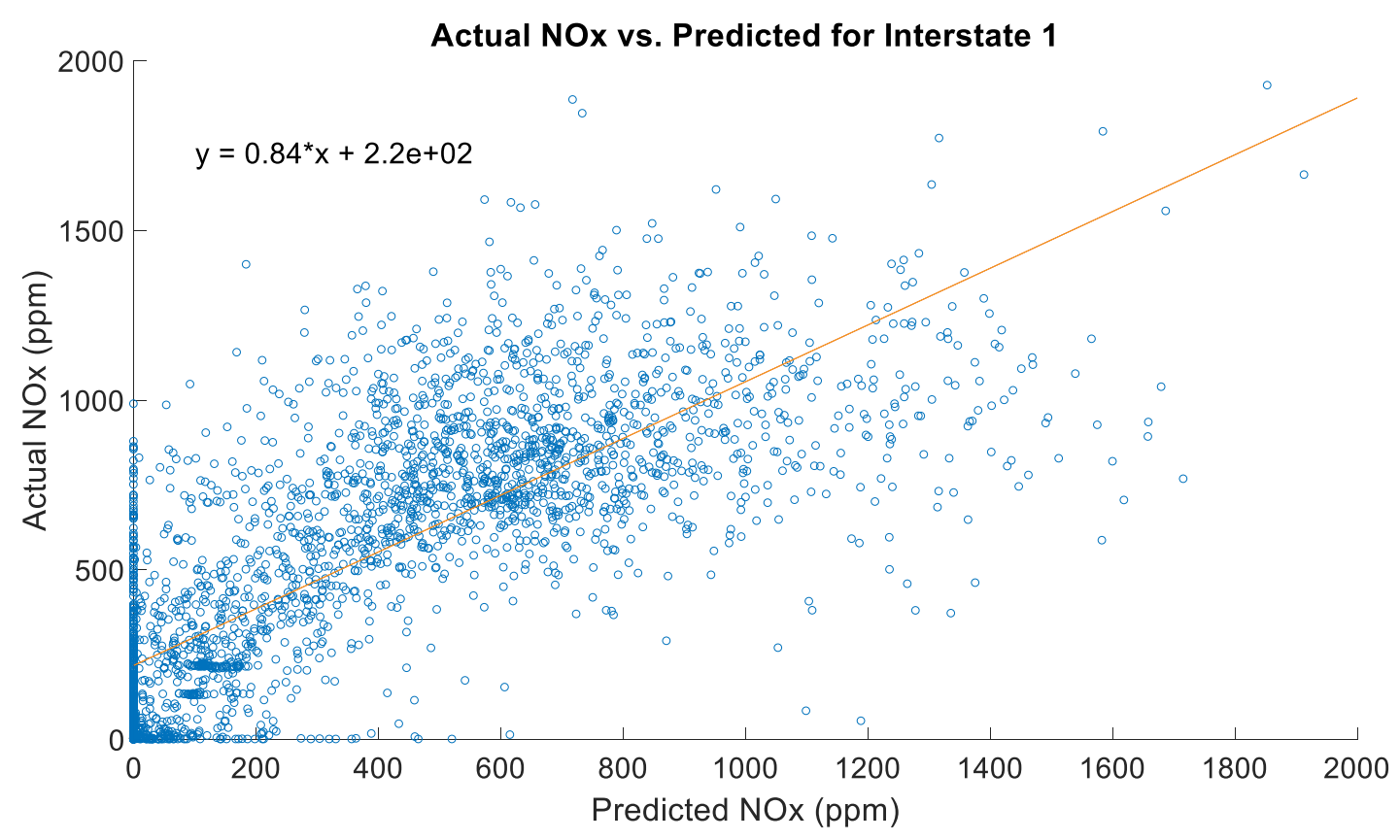

Figure 18- Scatterplot of Interstate 1 Validation Test with NN Trained with Training Data 1

Table 26-Interstate 1 Correlation Values

\begin{tabular}{|c|c|}
\hline \multicolumn{2}{|c|}{ Interstate 1 } \\
\hline \multicolumn{2}{|c|}{ R Value } \\
\cline { 1 - 1 } Validation Iterations & Average \\
\hline 0.85 & \multirow{2}{*}{0.80} \\
\cline { 1 - 1 } 0.81 & \\
\hline 0.75 & \\
\hline 0.82 & \\
\hline 0.77 & \\
\hline
\end{tabular}

Table 27 shows that other than a few exceptions, the error is generally between fifteen and thirty percent for all areas of the test. 
Table 27-Interstate 1-200 Second Errors

\begin{tabular}{|l|r|r|r|r|r|}
\hline \multicolumn{7}{|c|}{ Interstate 1 with Training Data Set 1 } \\
\hline & \multicolumn{1}{|c|}{ 200 Second Percent Errors } \\
\hline Time (s) & Test 1 & \multicolumn{1}{c|}{ Test 2 } & \multicolumn{1}{c|}{ Test 3 } & \multicolumn{1}{l|}{ Test 4 } & \multicolumn{1}{c|}{ Test 5 } \\
\hline $1-201$ & 5.33 & 2.83 & 3.68 & 9.72 & 4.41 \\
\hline $201-401$ & 2.99 & 12.03 & 14.57 & 20.99 & 15.84 \\
\hline $401-601$ & 31.71 & 24.19 & 19.66 & 22.82 & 21.70 \\
\hline $601-801$ & 34.60 & 26.76 & 21.48 & 26.76 & 33.45 \\
\hline $801-1001$ & 29.76 & 22.51 & 30.53 & 37.61 & 29.23 \\
\hline $1001-1201$ & 7.10 & 6.70 & 25.39 & 40.83 & 5.68 \\
\hline $1201-1401$ & 18.18 & 9.91 & 16.91 & 18.07 & 3.59 \\
\hline $1401-1601$ & 23.25 & 23.81 & 17.83 & 33.66 & 16.68 \\
\hline $1601-1801$ & 31.65 & 21.61 & 27.05 & 25.70 & 24.59 \\
\hline $1801-2001$ & 33.33 & 25.66 & 18.95 & 19.87 & 29.44 \\
\hline $2001-2201$ & 29.10 & 19.65 & 28.95 & 42.74 & 23.04 \\
\hline $2201-2401$ & 44.75 & 27.71 & 28.39 & 42.13 & 28.88 \\
\hline $2401-2601$ & 24.47 & 18.42 & 39.20 & 15.05 & 18.57 \\
\hline $2601-2801$ & 27.42 & 4.41 & 19.30 & 8.05 & 15.08 \\
\hline
\end{tabular}

\subsubsection{INTERSTATE 2}

Interstate 2 had similar error results (Table 28) to Interstate 1; however, it can be seen in Table 29 that there is less correlation between the predicted and actual for Interstate 2 (Figure 19).

Table 28-Interstate 2 Validation Test Errors when Trained with Training Data 1

\begin{tabular}{|c|c|}
\hline \multicolumn{2}{|c|}{ Interstate 2 } \\
\cline { 1 - 1 } Percent Error \\
\cline { 1 - 1 } Validation Iterations & Average \\
\cline { 1 - 1 } 13.36 & \multirow{2}{*}{20.67} \\
\cline { 1 - 1 } 42.87 & \\
\cline { 1 - 1 } 16.51 & \\
\cline { 1 - 1 } 13.32 & \\
\cline { 1 - 1 } 17.29 & \\
\hline
\end{tabular}




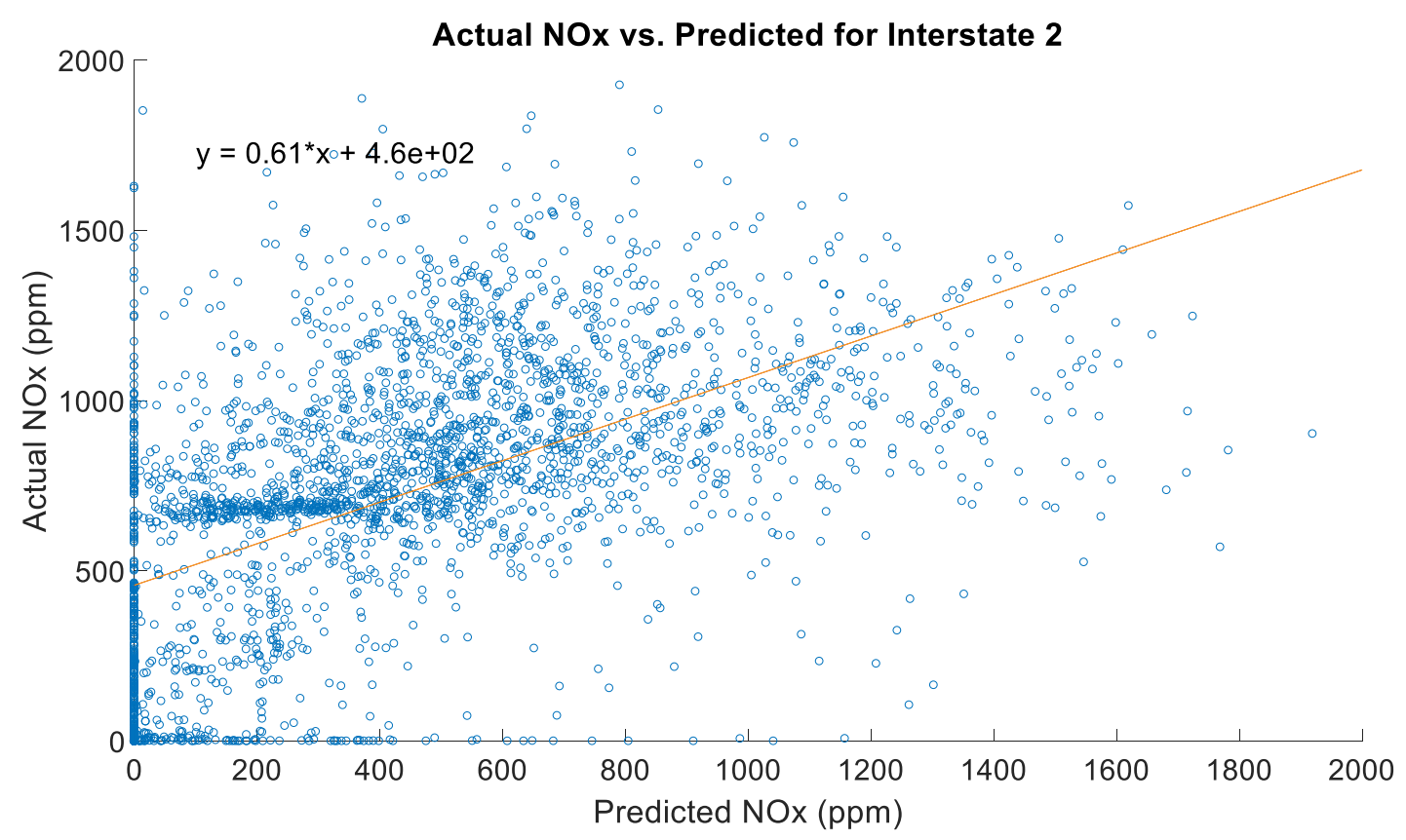

Figure 19- Scatterplot of Interstate 2 Validation Test with NN Trained with Training Data 1

Table 29-Interstate 2 Correlation Values

\begin{tabular}{|c|c|}
\hline \multicolumn{2}{|c|}{ Interstate 2 } \\
\cline { 1 - 1 } R Value \\
\cline { 1 - 1 } Validation Iterations & Average \\
\cline { 1 - 1 } 0.76 & \multirow{2}{*}{0.69} \\
\cline { 1 - 1 } 0.54 & \\
\cline { 1 - 1 } 0.69 & \\
\hline 0.74 & \\
\hline 0.74 & \\
\hline
\end{tabular}

Table 30 shows the errors in 200 second increments for Interstate 2 . This table shows the variability of a neural network each time it is trained. The errors are fairly consistent except for the third iteration. The third iteration yielded much higher errors than the other four tests. The average error for the third iteration was $42.89 \%$ compared to $14.05 \%, 14.89 \%, 15.96 \%$, and $13.26 \%$, for iterations one, two, four, and five, respectively. 
Table 30-Interstate 2-200 Second Errors

\begin{tabular}{|l|r|r|r|r|r|}
\hline \multicolumn{7}{|c|}{ Interstate 2 with Training Data Set 1 } \\
\hline & \multicolumn{1}{|c|}{ 200 Second Percent Errors } \\
\hline Time (s) & Test 1 & \multicolumn{1}{|c|}{ Test 2 } & \multicolumn{1}{c|}{ Test 3 } & \multicolumn{1}{c|}{ Test 4 } & \multicolumn{1}{c|}{ Test 5 } \\
\hline $1-201$ & 13.83 & 18.62 & 84.98 & 15.84 & 20.64 \\
\hline $201-401$ & 24.19 & 31.93 & 65.84 & 19.18 & 25.87 \\
\hline $401-601$ & 27.08 & 23.99 & 44.25 & 17.99 & 21.19 \\
\hline $601-801$ & 19.43 & 19.03 & 32.74 & 8.03 & 14.29 \\
\hline $801-1001$ & 13.42 & 14.30 & 47.31 & 21.65 & 13.37 \\
\hline $1001-1201$ & 9.09 & 7.34 & 34.53 & 14.34 & 8.47 \\
\hline $1201-1401$ & 8.22 & 5.83 & 58.35 & 28.43 & 5.36 \\
\hline $1401-1601$ & 2.54 & 1.93 & 26.32 & 11.22 & 4.66 \\
\hline $1601-1801$ & 12.30 & 15.13 & 16.69 & 3.63 & 10.19 \\
\hline $1801-2001$ & 20.74 & 18.72 & 19.28 & 12.26 & 12.80 \\
\hline $2001-2201$ & 21.56 & 17.35 & 45.10 & 23.22 & 20.23 \\
\hline $2201-2401$ & 4.46 & 6.77 & 42.90 & 25.15 & 12.44 \\
\hline $2401-2601$ & 5.81 & 12.26 & 39.34 & 6.58 & 2.88 \\
\hline
\end{tabular}

\subsection{TRaining DATA Set 2}

In this section of the results, the validation tests with the neural network trained with the Combined Training Data, training data set 2, will be analyzed. The percent error values, correlation values, and 200 second increment errors will be displayed for each test. The plots for the predicted values versus the actual values can be found in Appendix $\mathrm{H}$.

\subsubsection{HIGHWAY 2}

It was found that using training data set 2 caused an increase in error for Highway 2, which can be seen in Table 31. The prediction results are still below eleven percent error for all five iterations, but are higher than when tested with training data set 1. 
Table 31- Highway 2 Validation Test Errors when Trained with Training Data 2

\begin{tabular}{|c|c|}
\hline \multicolumn{2}{|c|}{ Highway 2 } \\
\cline { 1 - 1 } Percent Error \\
\cline { 1 - 1 } Validation Iterations & Average \\
\hline 7.42 & \multirow{2}{*}{9.40} \\
\cline { 1 - 1 } 9.38 & \\
\cline { 1 - 1 } 10.85 & \\
\cline { 1 - 1 } 10.83 & \\
\cline { 1 - 1 } 8.50 & \\
\hline
\end{tabular}

Figure 20 shows the plot of correlation between predicted and actual values for Highway 2.

Table 32 lists the correlation values for all five iterations. The average correlation is 0.76 which is a moderate-strong value.

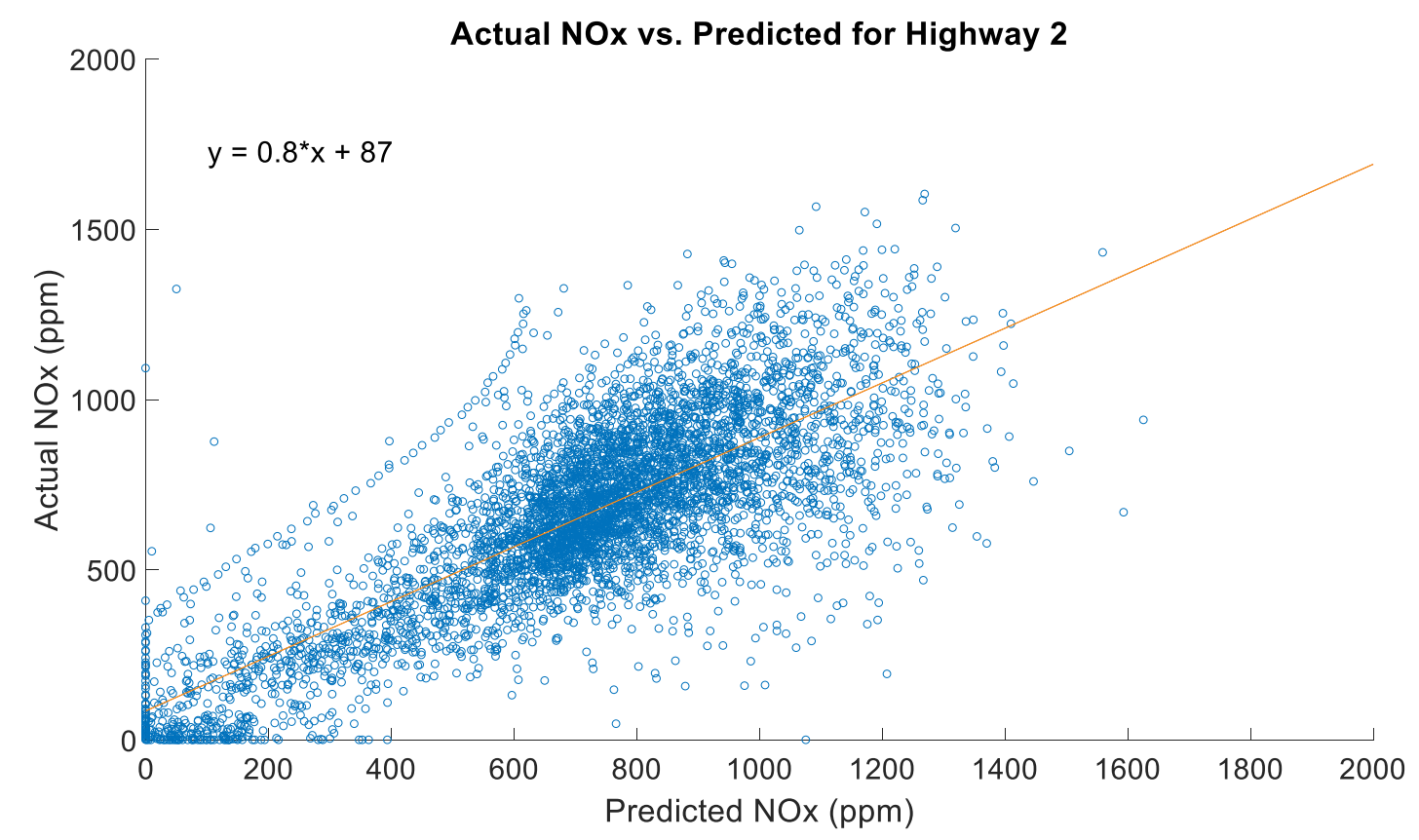

Figure 20-Scatterplot of Highway 2 Validation Test with NN Trained with Training Data 2 


\begin{tabular}{|c|c|}
\hline \multicolumn{2}{|c|}{ Highway 2 } \\
\hline \multicolumn{2}{|c|}{ R Value } \\
\cline { 1 - 1 } Validation Iterations & Average \\
\hline 0.76 & \multirow{3}{*}{0.762} \\
\cline { 1 - 1 } 0.78 & \\
\hline 0.77 & \\
\hline 0.75 & \\
\hline 0.75 & \\
\hline
\end{tabular}

Table 33 shows the errors in 200 second increments for Highway 2. It can be seen that there are certain areas where the error increases from the results in Section 4.2.1, but also a number of areas still have predictions under five percent. 
Table 33-Highway 2 Training Data 2-200 Second Errors

\begin{tabular}{|l|r|r|r|r|r|}
\hline \multicolumn{7}{|c|}{ Highway 2 with Training Data Set 2 } \\
\hline & \multicolumn{1}{|c|}{ 200 Second Percent Errors } \\
\hline Time (s) & \multicolumn{1}{|c|}{ Test 1 } & \multicolumn{1}{c|}{ Test 2 } & \multicolumn{1}{c|}{ Test 3 } & \multicolumn{1}{c|}{ Test 4 } & \multicolumn{1}{c|}{ Test 5 } \\
\hline $1-201$ & 11.27 & 21.73 & 26.61 & 26.10 & 10.93 \\
\hline $201-401$ & 16.69 & 23.00 & 27.14 & 29.36 & 16.02 \\
\hline $401-601$ & 7.51 & 14.91 & 16.55 & 18.03 & 11.26 \\
\hline $601-801$ & 7.81 & 12.78 & 14.57 & 14.48 & 10.37 \\
\hline $801-1001$ & 8.92 & 7.91 & 8.12 & 9.60 & 10.08 \\
\hline $1001-1201$ & 0.70 & 0.82 & 0.54 & 0.37 & 2.65 \\
\hline $1201-1401$ & 1.14 & 3.07 & 2.07 & 1.94 & 3.85 \\
\hline $1401-1601$ & 1.26 & 2.16 & 1.18 & 2.00 & 5.87 \\
\hline $1601-1801$ & 17.56 & 11.51 & 12.02 & 16.80 & 18.24 \\
\hline $1801-2001$ & 3.15 & 4.44 & 3.38 & 2.75 & 11.47 \\
\hline $2001-2201$ & 29.76 & 14.53 & 24.04 & 24.90 & 27.43 \\
\hline $2201-2401$ & 2.13 & 4.20 & 1.02 & 0.81 & 5.05 \\
\hline $2401-2601$ & 4.24 & 2.85 & 4.07 & 3.43 & 2.38 \\
\hline $2601-2801$ & 0.82 & 0.74 & 1.18 & 0.30 & 0.24 \\
\hline $2801-3001$ & 4.63 & 4.73 & 5.18 & 4.15 & 6.23 \\
\hline $3001-3201$ & 8.44 & 12.85 & 14.12 & 12.10 & 16.22 \\
\hline $3201-3401$ & 6.42 & 11.32 & 11.26 & 10.90 & 14.45 \\
\hline $3401-3601$ & 6.40 & 7.34 & 7.38 & 7.45 & 6.73 \\
\hline $3601-3801$ & 6.23 & 6.97 & 7.82 & 7.95 & 5.90 \\
\hline $3801-4001$ & 7.09 & 6.73 & 8.52 & 5.72 & 7.43 \\
\hline $4001-4201$ & 6.96 & 10.44 & 9.50 & 8.71 & 7.29 \\
\hline $4201-4401$ & 15.52 & 14.55 & 16.84 & 13.08 & 11.66 \\
\hline $4401-4601$ & 10.28 & 12.34 & 12.94 & 12.39 & 4.52 \\
\hline $4601-4801$ & 7.75 & 12.34 & 13.68 & 18.04 & 11.23 \\
\hline $4801-5001$ & 9.85 & 6.88 & 18.97 & 16.87 & 11.48 \\
\hline & & & & & \\
\hline
\end{tabular}

\subsubsection{HIGHWAY 3}

The Highway 3 validation test had the biggest effect from switching the training data. By switching the training data Highway 3 changed from the best performing validation test to the worst performing validation test. The percent error increased from $0.926 \%$ (Table 13) to $37.24 \%$ (Table 34). The correlation coefficient goes from 0.83 (Table 14) to 0.56 (Table 35). This shift in performance can be contributed to the test data compared to the training data. Highway 3 data 
is similar to the data in training data 1 , but training data 2 includes data from a number of different locations. A plot of the predicted versus actual values can be seen in Figure 21 .

Table 34- Highway 3 Validation Test Errors when Trained with Training Data 2

\begin{tabular}{|c|c|}
\hline \multicolumn{2}{|c|}{ Highway 3} \\
\hline \multicolumn{2}{|c|}{ Percent Error } \\
\cline { 1 - 1 } Validation Iterations & Average \\
\hline 38.80 & \multirow{3}{*}{37.24} \\
\cline { 1 - 1 } 41.10 & \\
\cline { 1 - 1 } 55.76 & \\
\hline 4.060 & \\
\hline 46.47 & \\
\hline
\end{tabular}

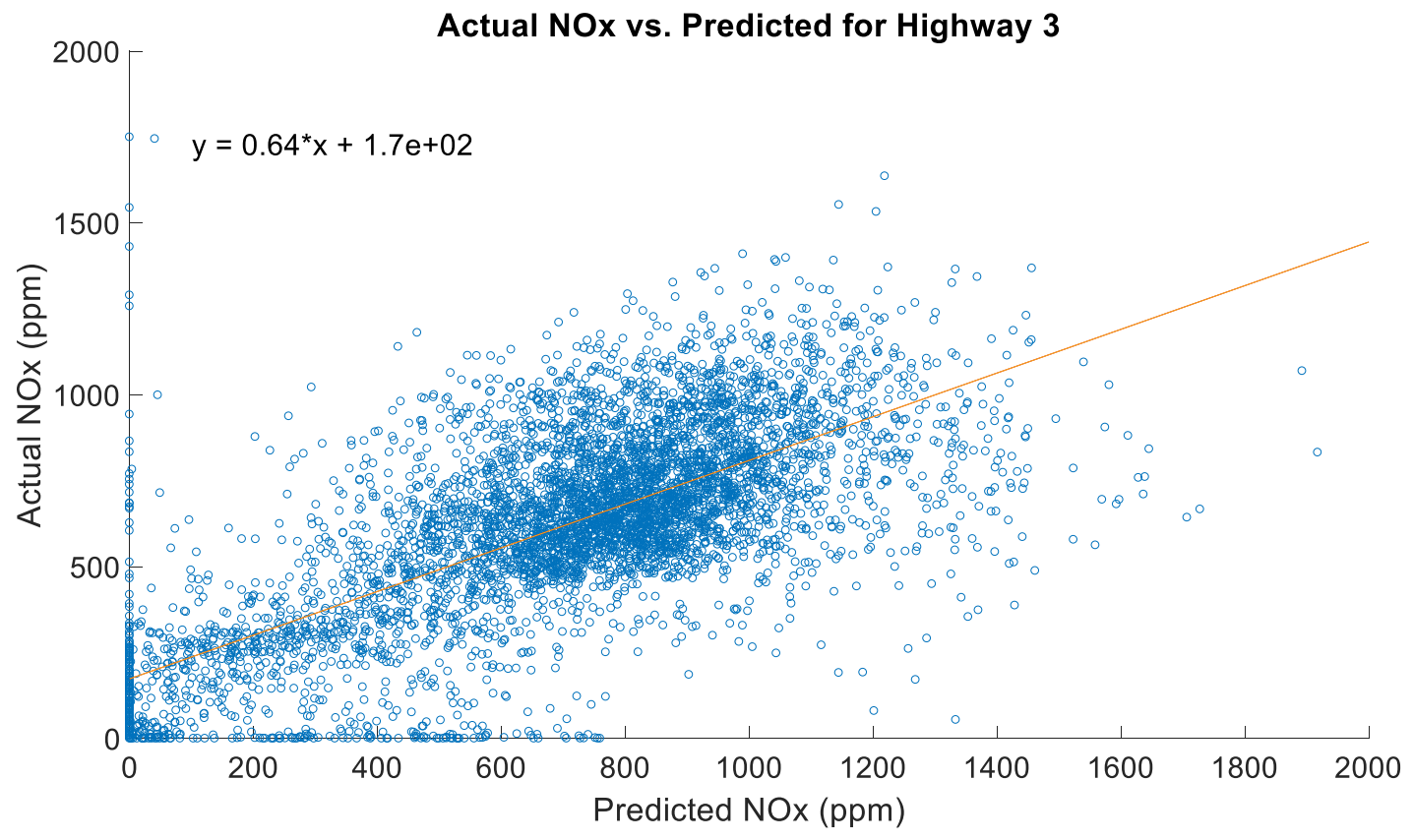

Figure 21- Scatterplot of Highway 3 Validation Test with NN Trained with Training Data 2 
Table 35-Highway 3 Training Data 2 Correlation Values

\begin{tabular}{|c|c|}
\hline \multicolumn{2}{|c|}{ Highway 3} \\
\hline \multicolumn{2}{|c|}{ R Value } \\
\cline { 1 - 1 } Validation Iterations & Average \\
\hline 0.53 & \multirow{2}{*}{0.56} \\
\cline { 1 - 1 } 0.5 & \\
\cline { 1 - 1 } 0.42 & \\
\hline 0.73 & \\
\hline 0.62 & \\
\hline
\end{tabular}

Table 36 shows the 200 second increment errors throughout the Highway 3 test. Iteration 4 is another example of how the results can vary based on training iterations. The results for iteration four are much better than the other iterations, but overall the performance of the prediction was poor compared to the other validation tests. 
Table 36-Highway 3 Training Data 2-200 Second Errors

\begin{tabular}{|l|r|r|r|r|r|}
\hline \multicolumn{7}{|c|}{ Highway 3 with Training Data Set 2 } \\
\hline & \multicolumn{1}{|c|}{ 200 Second Percent Errors } \\
\hline Time (s) & \multicolumn{1}{|c|}{ Test 1 } & \multicolumn{1}{c|}{ Test 2 } & \multicolumn{1}{c|}{ Test 3 } & \multicolumn{1}{c|}{ Test 4 } & \multicolumn{1}{c|}{ Test 5 } \\
\hline $1-201$ & 14.46 & 16.15 & 17.78 & 6.49 & 10.46 \\
\hline $201-401$ & 18.78 & 25.42 & 26.17 & 4.88 & 27.83 \\
\hline $401-601$ & 16.64 & 18.61 & 24.84 & 6.49 & 24.36 \\
\hline $601-801$ & 24.72 & 13.86 & 35.44 & 6.33 & 25.68 \\
\hline $801-1001$ & 20.41 & 19.43 & 32.51 & 14.18 & 29.08 \\
\hline $1001-1201$ & 24.54 & 26.04 & 35.37 & 13.84 & 25.07 \\
\hline $1201-1401$ & 21.14 & 24.58 & 28.55 & 7.04 & 28.47 \\
\hline $1401-1601$ & 16.00 & 21.71 & 24.50 & 10.53 & 26.95 \\
\hline $1601-1801$ & 22.03 & 30.81 & 30.54 & 1.55 & 31.62 \\
\hline $1801-2001$ & 26.52 & 34.77 & 36.13 & 5.45 & 37.66 \\
\hline $2001-2201$ & 23.93 & 30.50 & 37.78 & 2.19 & 37.85 \\
\hline $2201-2401$ & 54.28 & 50.14 & 63.64 & 14.17 & 53.47 \\
\hline $2401-2601$ & 106.00 & 108.73 & 132.14 & 43.86 & 71.58 \\
\hline $2601-2801$ & 48.25 & 47.42 & 56.00 & 20.40 & 47.86 \\
\hline $2801-3001$ & 36.77 & 46.39 & 51.27 & 6.42 & 50.80 \\
\hline $3001-3201$ & 36.71 & 36.04 & 56.39 & 4.89 & 54.06 \\
\hline $3201-3401$ & 41.47 & 44.37 & 60.81 & 6.55 & 50.82 \\
\hline $3401-3601$ & 40.00 & 41.94 & 60.07 & 8.33 & 50.70 \\
\hline $3601-3801$ & 38.99 & 42.43 & 63.66 & 1.79 & 63.67 \\
\hline $3801-4001$ & 31.51 & 28.99 & 54.56 & 2.41 & 49.76 \\
\hline $4001-4201$ & 32.05 & 29.45 & 55.40 & 2.69 & 51.39 \\
\hline $4201-4401$ & 47.35 & 39.51 & 64.85 & 3.32 & 61.08 \\
\hline $4401-4601$ & 36.95 & 41.88 & 62.16 & 5.62 & 61.35 \\
\hline $4601-4801$ & 36.06 & 41.62 & 57.61 & 8.99 & 57.39 \\
\hline $4801-5001$ & 40.71 & 46.47 & 54.08 & 6.32 & 54.25 \\
\hline & & & & & \\
\hline
\end{tabular}

\subsubsection{REGIONAL}

The Regional test using training data 2 begins to show the improvement of the prediction

for all the tests except for the Highway tests. The average error of 6.91 percent can be seen in Table 37. 
Table 37-Regional Validation Test Errors when Trained with Training Data 2

\begin{tabular}{|c|c|}
\hline \multicolumn{2}{|c|}{ Regional } \\
\hline \multicolumn{2}{|c|}{ Percent Error } \\
\cline { 1 - 1 } Validation Iterations & Average \\
\hline 6.24 & \multirow{2}{*}{6.91} \\
\cline { 1 - 1 } 6.74 & \\
\cline { 1 - 1 } 10.2 & \\
\hline 6.50 & \\
\hline 4.87 & \\
\hline
\end{tabular}

Figure 22 shows the correlation between predicted and actual values for the Regional test. The correlation values for all five iterations can be seen in Table 38. The average correlation coefficient is 0.82 , which means there is a strong correlation between the predicted and actual values.

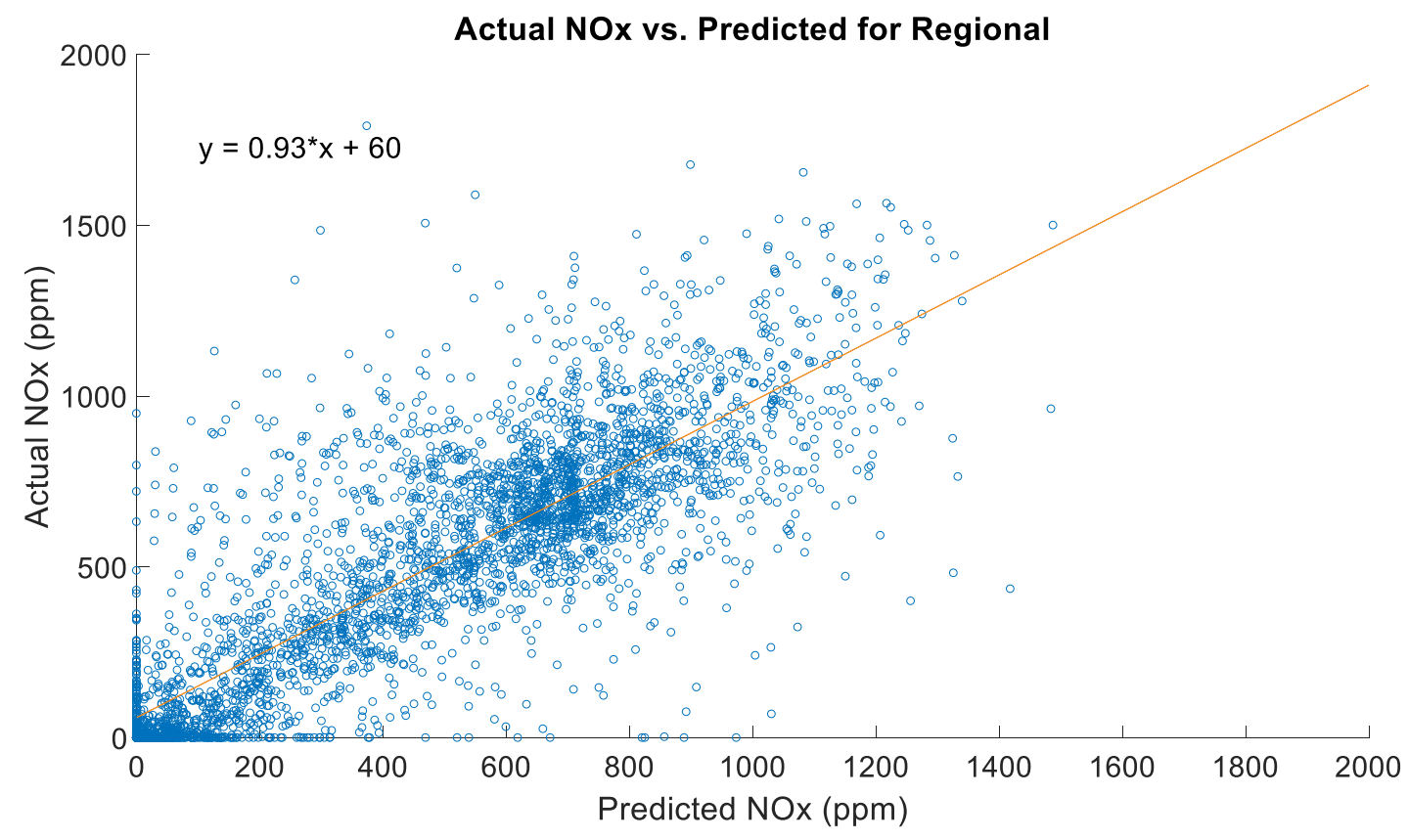

Figure 22- Scatterplot of Regional Validation Test with NN Trained with Training Data 2 
Table 38-Regional Training Data 2 Correlation Values

\begin{tabular}{|c|c|}
\hline \multicolumn{2}{|c|}{ Regional } \\
\hline \multicolumn{2}{|c|}{ R Value } \\
\cline { 1 - 1 } Validation Iterations & Average \\
\hline 0.82 & \multirow{2}{*}{0.82} \\
\cline { 1 - 1 } 0.85 & \\
\hline 0.78 & \\
\hline 0.82 & \\
\hline 0.82 & \\
\hline
\end{tabular}

Looking at Table 39, extremely high errors can be seen between 2000-2600 seconds.

Outside of this range the error appears to be below five percent, with a few exceptions.

Table 39-Regional Training Data 2-200 Second Errors

\begin{tabular}{|l|r|r|r|r|r|}
\hline \multicolumn{7}{|c|}{ Regional with Training Data Set 2 } \\
\hline & \multicolumn{1}{|c|}{ 200 Second Percent Errors } \\
\hline Time (s) & Test 1 & Test 2 & \multicolumn{1}{c|}{ Test 3 } & \multicolumn{1}{c|}{ Test 4 } & \multicolumn{1}{c|}{ Test 5 } \\
\hline $1-201$ & 10.87 & 13.70 & 2.60 & 18.04 & 7.39 \\
\hline $201-401$ & 20.01 & 12.08 & 19.26 & 12.95 & 13.20 \\
\hline $401-601$ & 3.54 & 3.10 & 0.49 & 4.18 & 2.80 \\
\hline $601-801$ & 1.96 & 2.31 & 12.11 & 15.32 & 16.32 \\
\hline $801-1001$ & 1.31 & 1.06 & 0.43 & 0.20 & 1.58 \\
\hline $1001-1201$ & 2.44 & 1.42 & 0.94 & 1.32 & 0.61 \\
\hline $1201-1401$ & 8.16 & 6.28 & 11.90 & 7.40 & 15.13 \\
\hline $1401-1601$ & 1.34 & 1.08 & 4.80 & 3.62 & 6.25 \\
\hline $1601-1801$ & 5.24 & 4.28 & 18.26 & 16.75 & 3.41 \\
\hline $1801-2001$ & 8.17 & 0.67 & 8.44 & 5.81 & 3.81 \\
\hline $2001-2201$ & 23.92 & 18.25 & 42.12 & 14.07 & 10.25 \\
\hline $2201-2401$ & 28.23 & 20.51 & 37.36 & 20.02 & 9.82 \\
\hline $2401-2601$ & 66.51 & 49.51 & 106.93 & 79.39 & 81.77 \\
\hline $2601-2801$ & 4.92 & 12.71 & 20.18 & 9.70 & 9.90 \\
\hline $2801-3001$ & 4.38 & 0.94 & 6.93 & 2.60 & 3.80 \\
\hline $3001-3201$ & 3.91 & 4.01 & 4.15 & 7.90 & 6.42 \\
\hline $3201-3401$ & 2.44 & 11.00 & 8.13 & 8.69 & 10.55 \\
\hline $3401-3601$ & 7.48 & 2.67 & 6.44 & 3.43 & 5.20 \\
\hline
\end{tabular}




\subsubsection{LOCAL}

The local validation test produced very inconsistent results. Table 40 shows the errors for each of the five iterations. The error values range from 1.25-16.82.

Table 40- Local Validation Test Errors when Trained with Training Data 2

\begin{tabular}{|c|c|}
\hline \multicolumn{2}{|c|}{ Local } \\
\hline \multicolumn{2}{|c|}{ Percent Error } \\
\cline { 1 - 1 } Validation Iterations & Average \\
\hline 2.00 & \multirow{2}{*}{8.69} \\
\cline { 1 - 1 } 1.25 & \\
\cline { 1 - 1 } 12.43 & \\
\hline 16.82 & \\
\hline 10.93 & \\
\hline
\end{tabular}

A plot of the correlation between the actual and predicted value can be seen in Figure 23 .

The values for the correlation are listed in Table 41.

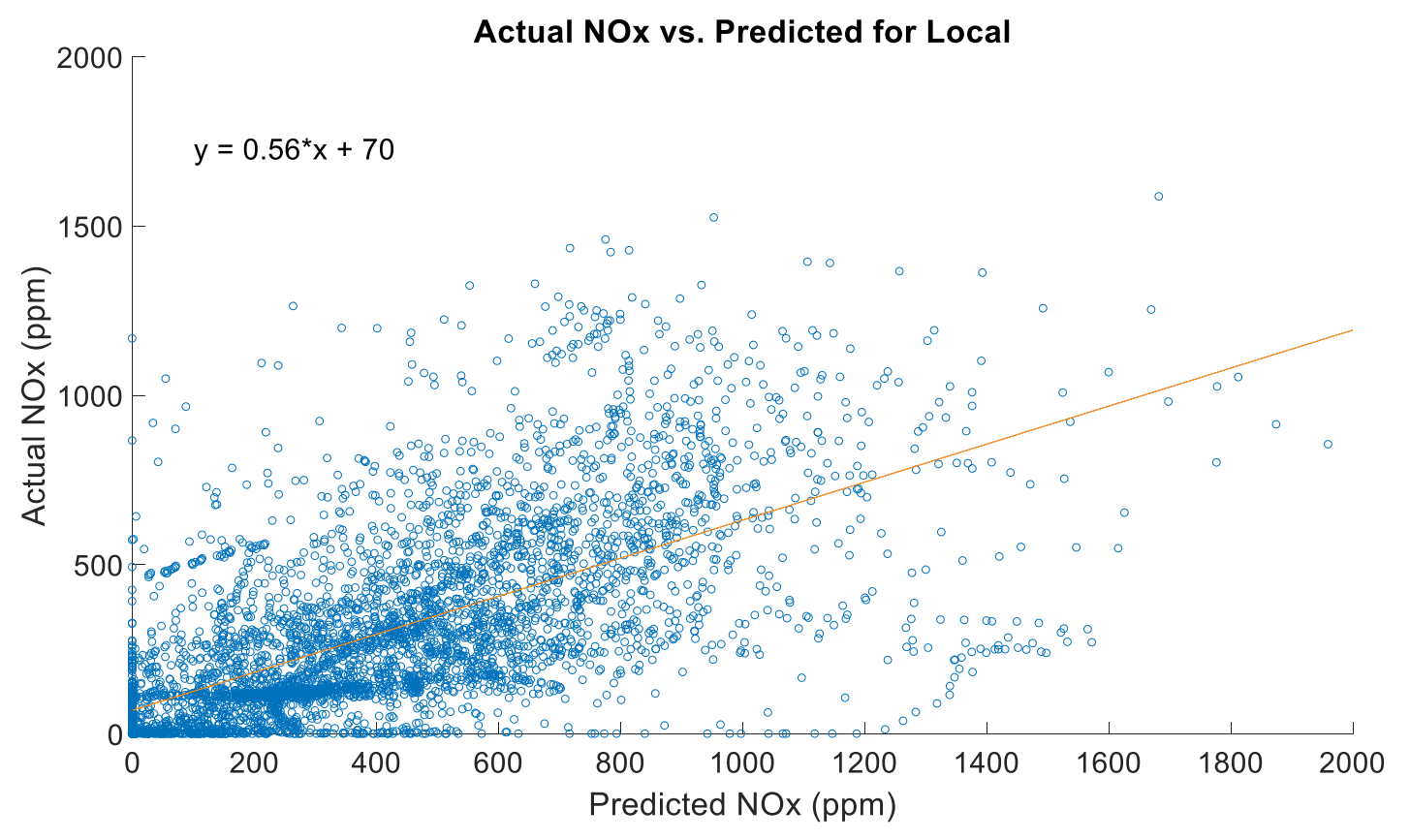

Figure 23- Scatterplot of Local Validation Test with NN Trained with Training Data 2 
Table 41-Local Training Data 2 Correlation Values

\begin{tabular}{|c|c|}
\hline \multicolumn{2}{|c|}{ Local } \\
\hline \multicolumn{2}{|c|}{ R Value } \\
\cline { 1 - 1 } Validation Iterations & Average \\
\hline 0.71 & \multirow{3}{*}{0.72} \\
\cline { 1 - 1 } 0.77 & \\
\cline { 1 - 1 } 0.77 & \\
\hline 0.69 & \\
\hline 0.68 & \\
\hline
\end{tabular}

Table 42 shows how inconsistent the results were for the Local test. In the first 200 seconds two iterations had results of three percent and lower, while another iteration had a 34.48 percent error. In the $2600-2800$ section two iterations have error of nineteen percent and higher while two other iterations have an error of 1.95 and below. Section 3400-3600 has a range of errors from 28.19 to 68.18 , and the worst section is $2800-3000$ where the errors range between 8.21-85.84. 
Table 42-Local Training Data 2-200 Second Errors

\begin{tabular}{|l|r|r|r|r|r|}
\hline \multicolumn{7}{|c|}{ Local with Training Data Set 2 } \\
\hline & \multicolumn{1}{|c|}{ 200 Second Percent Errors } \\
\hline Time (s) & Test 1 & \multicolumn{1}{|c|}{ Test 2 } & \multicolumn{1}{|c|}{ Test 3 } & \multicolumn{1}{c|}{ Test 4 } & \multicolumn{1}{c|}{ Test 5 } \\
\hline $1-201$ & 8.53 & 12.61 & 34.48 & 3.05 & 2.16 \\
\hline $201-401$ & 9.12 & 1.13 & 14.71 & 11.81 & 10.89 \\
\hline $401-601$ & 6.80 & 9.61 & 15.36 & 14.13 & 17.65 \\
\hline $601-801$ & 5.24 & 7.68 & 6.00 & 7.05 & 10.26 \\
\hline $801-1001$ & 1.16 & 1.77 & 5.35 & 1.19 & 2.91 \\
\hline $1001-1201$ & 0.06 & 0.84 & 10.29 & 0.55 & 5.37 \\
\hline $1201-1401$ & 10.83 & 0.95 & 9.55 & 6.06 & 6.91 \\
\hline $1401-1601$ & 1.06 & 5.67 & 6.62 & 11.46 & 30.72 \\
\hline $1601-1801$ & 28.70 & 32.45 & 9.27 & 12.96 & 47.37 \\
\hline $1801-2001$ & 40.44 & 10.86 & 36.89 & 46.18 & 14.41 \\
\hline $2001-2201$ & 14.97 & 16.00 & 10.29 & 19.94 & 2.71 \\
\hline $2201-2401$ & 45.00 & 19.55 & 8.61 & 18.92 & 18.77 \\
\hline $2401-2601$ & 5.03 & 4.68 & 13.47 & 13.92 & 14.17 \\
\hline $2601-2801$ & 1.95 & 8.62 & 0.14 & 22.07 & 19.39 \\
\hline $2801-3001$ & 8.21 & 27.55 & 45.35 & 85.84 & 47.89 \\
\hline $3001-3201$ & 69.28 & 61.03 & 14.61 & 9.65 & 73.02 \\
\hline $3201-3401$ & 4.69 & 11.79 & 11.67 & 37.70 & 46.78 \\
\hline $3401-3601$ & 34.32 & 36.39 & 36.04 & 68.18 & 28.19 \\
\hline $3601-3801$ & 40.80 & 10.93 & 26.57 & 32.41 & 22.62 \\
\hline $3801-4001$ & 50.61 & 12.38 & 1.41 & 26.04 & 18.83 \\
\hline $4001-4201$ & 25.10 & 25.77 & 6.16 & 4.68 & 0.78 \\
\hline $4201-4401$ & 11.38 & 14.65 & 37.42 & 38.94 & 26.44 \\
\hline
\end{tabular}

\subsubsection{NEARDOCK}

The Neardock validation test showed decent results. The average of the five iterations was a 6.47 percent error, which can be seen in Table 43.

Table 43- Neardock Validation Test Errors when Trained with Training Data 2

\begin{tabular}{|c|c|}
\hline \multicolumn{2}{|c|}{ Neardock } \\
\hline \multicolumn{2}{|c|}{ Percent Error } \\
\cline { 1 - 1 } Validation Iterations & Average \\
\hline 13.72 & \multirow{2}{*}{6.47} \\
\cline { 1 - 1 } 5.53 & \\
\cline { 1 - 1 } 0 & \\
\hline 3.4 & \\
\hline 9.71 &
\end{tabular}


Figure 24 shows the correlation plot for the predicted to actual values for the Neardock test. The correlation values can be seen in Table 44 . The average correlation value was 0.79 which shows a strong correlation.

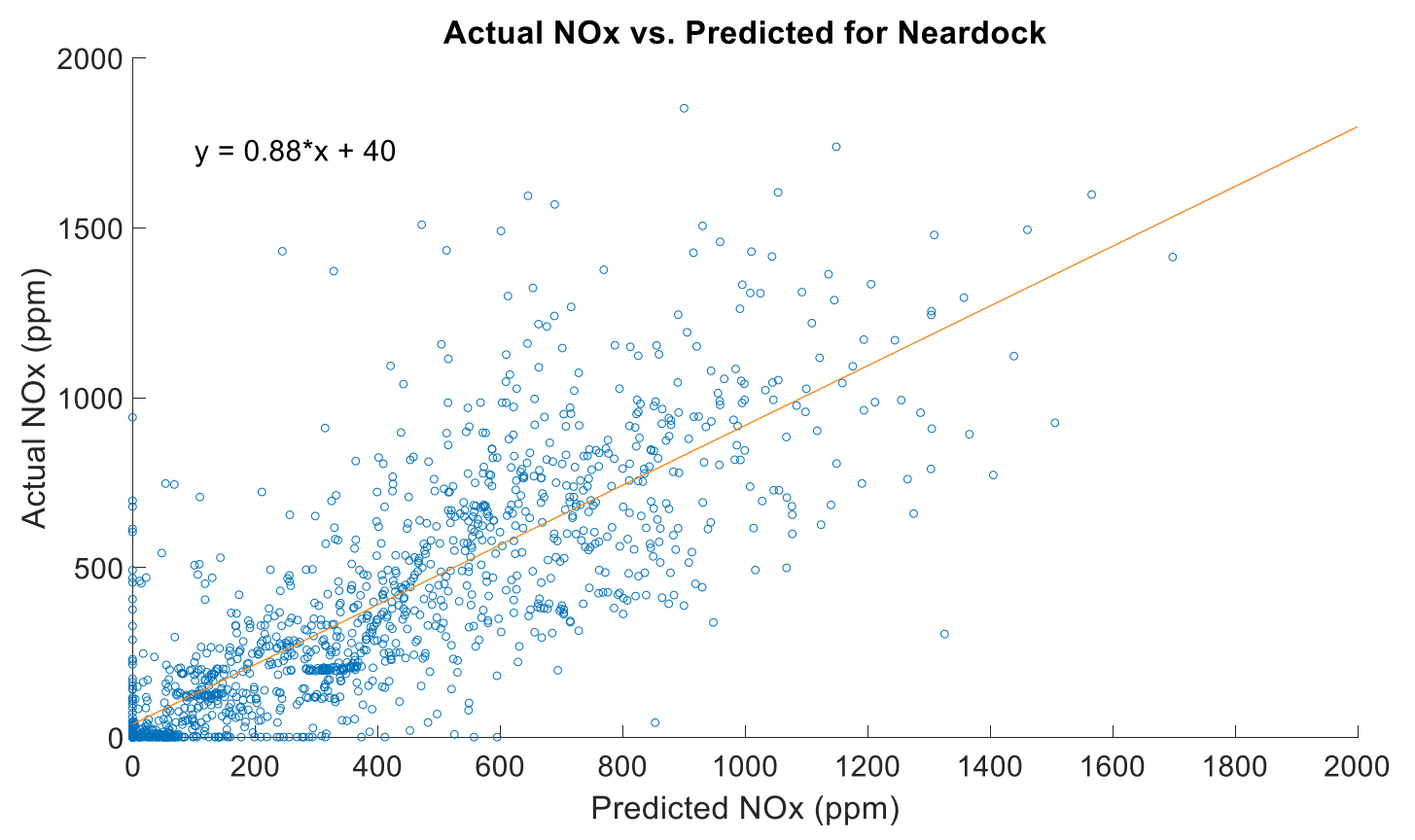

Figure 24- Scatterplot of Neardock Validation Test with NN Trained with Training Data 2

Table 44-Neardock Training Data 2 Correlation Values

\begin{tabular}{|c|c|}
\hline \multicolumn{2}{|c|}{ Neardock } \\
\hline \multicolumn{2}{|c|}{ R Value } \\
\cline { 1 - 1 } Validation Iterations & Average \\
\hline 0.8 & \multirow{2}{*}{0.79} \\
\cline { 1 - 1 } 0.83 & \\
\cline { 1 - 1 } 0.79 & \\
\hline 0.73 & \\
\hline 0.82 & \\
\hline
\end{tabular}

Table 45 shows the error in 200 second increments. The results for the first half of the test $(0-600 \mathrm{sec}$ ) resulted in errors less than five percent for every iteration. The last 400 seconds of the test (800-1200 sec.) show a major increase in error. There is an average increase of $19.25 \%$ between the first 600 seconds and the last 400 seconds. 
Table 45-Neardock Training Data 2-200 Second Errors

\begin{tabular}{|l|r|r|r|r|r|}
\hline \multicolumn{7}{|c|}{ Neardock with Training Data Set 2 } \\
\hline & \multicolumn{7}{|c|}{ 200 Second Percent Errors } \\
\hline Time (s) & Test 1 & \multicolumn{1}{|c|}{ Test 2 } & \multicolumn{1}{c|}{ Test 3 } & Test 4 & Test 5 \\
\hline $1-201$ & 4.54 & 2.80 & 1.22 & 4.59 & 0.07 \\
\hline $201-401$ & 1.59 & 1.87 & 0.42 & 0.44 & 0.76 \\
\hline $401-601$ & 1.16 & 0.47 & 2.94 & 0.05 & 1.51 \\
\hline $601-801$ & 0.74 & 6.55 & 6.01 & 20.85 & 1.78 \\
\hline $801-1001$ & 33.56 & 13.67 & 16.93 & 12.86 & 20.73 \\
\hline $1001-1201$ & 36.91 & 10.85 & 13.89 & 33.53 & 27.10 \\
\hline
\end{tabular}

\subsubsection{INTERSTATE 1}

Interstate 1 had the best results when the neural network was trained with training data

2 , in terms of percent error. The average error was 5.81, shown in Table 46.

Table 46-Interstate 1 Validation Test Errors when Trained with Training Data 2

\begin{tabular}{|c|c|}
\hline \multicolumn{2}{|c|}{ Interstate 1 } \\
\hline \multicolumn{2}{|c|}{ Percent Error } \\
\cline { 1 - 1 } Validation Iterations & Average \\
\hline 7.55 & \\
\cline { 1 - 1 } 5.02 & \multirow{2}{*}{5.81} \\
\cline { 1 - 1 } 6.8 & \\
\hline 4.24 & \\
\hline 5.43 & \\
\hline
\end{tabular}




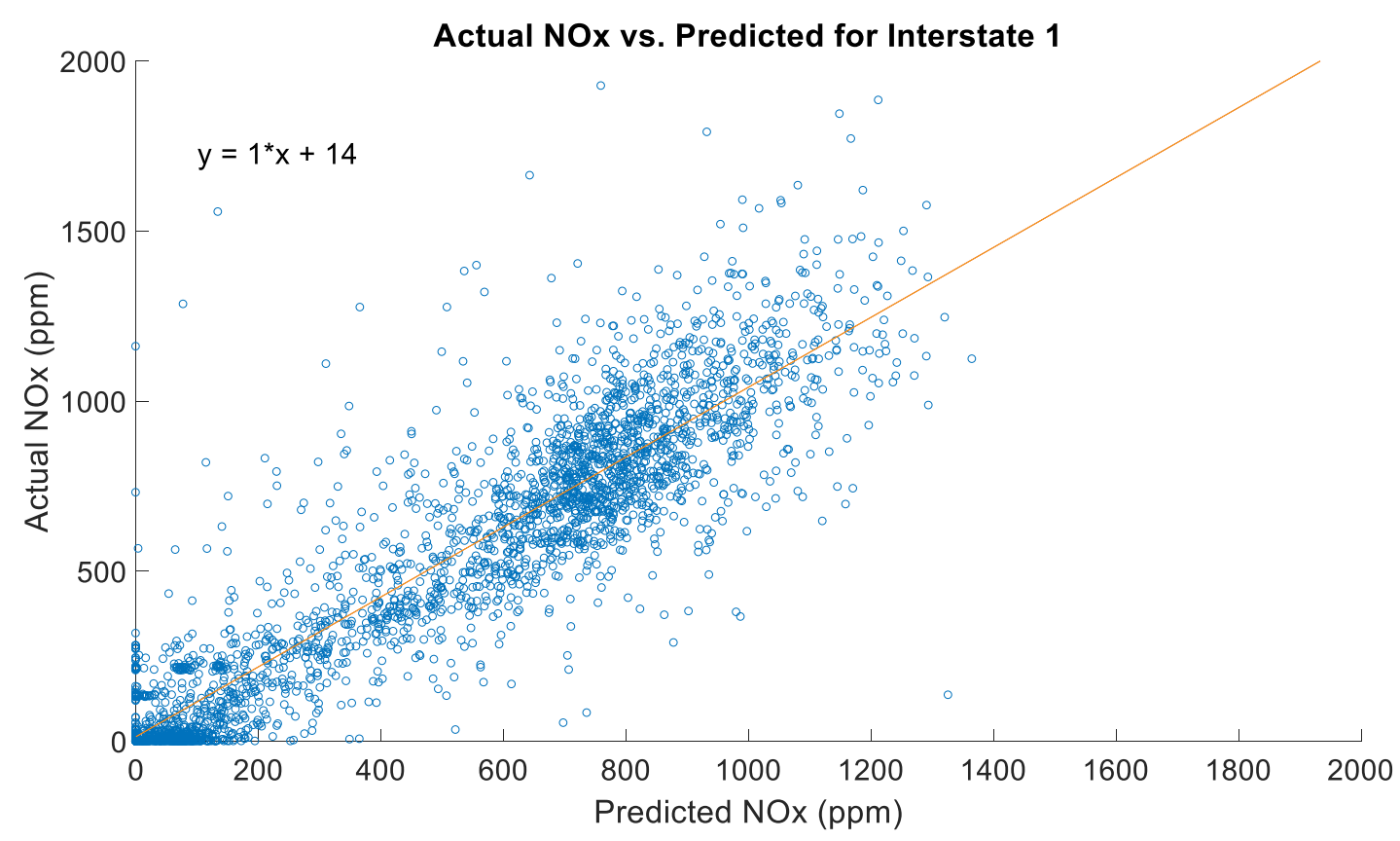

Figure 25-Scatterplot of Interstate 1 Validation Test with NN Trained with Training Data 2

Figure 25 shows the correlation between the predicted and actual values. The values for all five iterations can be seen in Table 47. The average correlation value was 0.90 which shows a very strong correlation between the actual and predicted values.

Table 47-Interstate 1 Training Data 2 Correlation Values

\begin{tabular}{|c|c|}
\hline \multicolumn{2}{|c|}{ Interstate 1} \\
\hline \multicolumn{2}{|c|}{ R Value } \\
\cline { 1 - 1 } Validation Iterations & Average \\
\hline 0.9 & \multirow{2}{*}{0.90} \\
\cline { 1 - 1 } 0.9 & \\
\cline { 1 - 1 } 0.91 & \\
\hline 0.91 & \\
\hline 0.88 & \\
\hline
\end{tabular}

Table 48 shows the error every 200 seconds throughout the length of the test. This table shows that other than the first and last 200 seconds of the test the errors are generally under ten percent. 
Table 48-Interstate 1 Training Data 2-200 Second Errors

\begin{tabular}{|l|r|r|r|r|r|}
\hline \multicolumn{7}{|c|}{ Interstate 1 with Training Data Set 2 } \\
\hline & \multicolumn{1}{|c|}{ 200 Second Percent Errors } \\
\hline Time (s) & \multicolumn{1}{|c|}{ Test 1 } & \multicolumn{1}{|c|}{ Test 2 } & \multicolumn{1}{|c|}{ Test 3 } & \multicolumn{1}{c|}{ Test 4 } & \multicolumn{1}{c|}{ Test 5 } \\
\hline $1-201$ & 9.52 & 9.95 & 14.45 & 13.95 & 33.32 \\
\hline $201-401$ & 4.47 & 6.22 & 2.17 & 2.94 & 2.24 \\
\hline $401-601$ & 1.99 & 2.95 & 2.29 & 2.74 & 2.19 \\
\hline $601-801$ & 6.12 & 5.04 & 7.02 & 4.39 & 4.88 \\
\hline $801-1001$ & 2.75 & 2.16 & 3.12 & 4.54 & 3.13 \\
\hline $1001-1201$ & 1.84 & 1.61 & 1.49 & 3.84 & 3.96 \\
\hline $1201-1401$ & 2.01 & 2.03 & 2.70 & 4.23 & 0.81 \\
\hline $1401-1601$ & 6.07 & 3.01 & 7.19 & 1.05 & 4.14 \\
\hline $1601-1801$ & 7.17 & 2.78 & 7.67 & 4.14 & 4.12 \\
\hline $1801-2001$ & 11.21 & 7.42 & 10.62 & 5.43 & 6.22 \\
\hline $2001-2201$ & 9.06 & 8.72 & 8.79 & 7.71 & 4.76 \\
\hline $2201-2401$ & 11.58 & 10.47 & 11.21 & 8.40 & 5.55 \\
\hline $2401-2601$ & 6.57 & 1.12 & 6.91 & 0.26 & 6.81 \\
\hline $2601-2801$ & 53.14 & 31.27 & 25.84 & 20.86 & 19.30 \\
\hline
\end{tabular}

\subsubsection{INTERSTATE 2}

Interstate 2 results resembled the results for Interstate 1 . The average error was 7.94, shown in Table 49. The correlation value is slightly lower for Interstate 1 , than it was for Interstate 2. The average value for Interstate 2 was 0.82 , which is still a strong correlation between the actual and predicted values. The values for the correlation for the five iterations can be seen in Table 50. A plot of the correlation can be seen in Figure 26.

Table 49-Interstate 2 Validation Test Errors when Trained with Training Data 2

\begin{tabular}{|c|c|}
\hline \multicolumn{2}{|c|}{ Interstate 2 } \\
\hline \multicolumn{2}{|c|}{ Percent Error } \\
\cline { 1 - 1 } Validation Iterations & Average \\
\cline { 1 - 1 } 6.54 & \multirow{2}{*}{7.94} \\
\cline { 1 - 1 } 9.58 & \\
\cline { 1 - 1 } 8.61 & \\
\cline { 1 - 1 } 9.66 & \\
\cline { 1 - 1 } 5.29 &
\end{tabular}




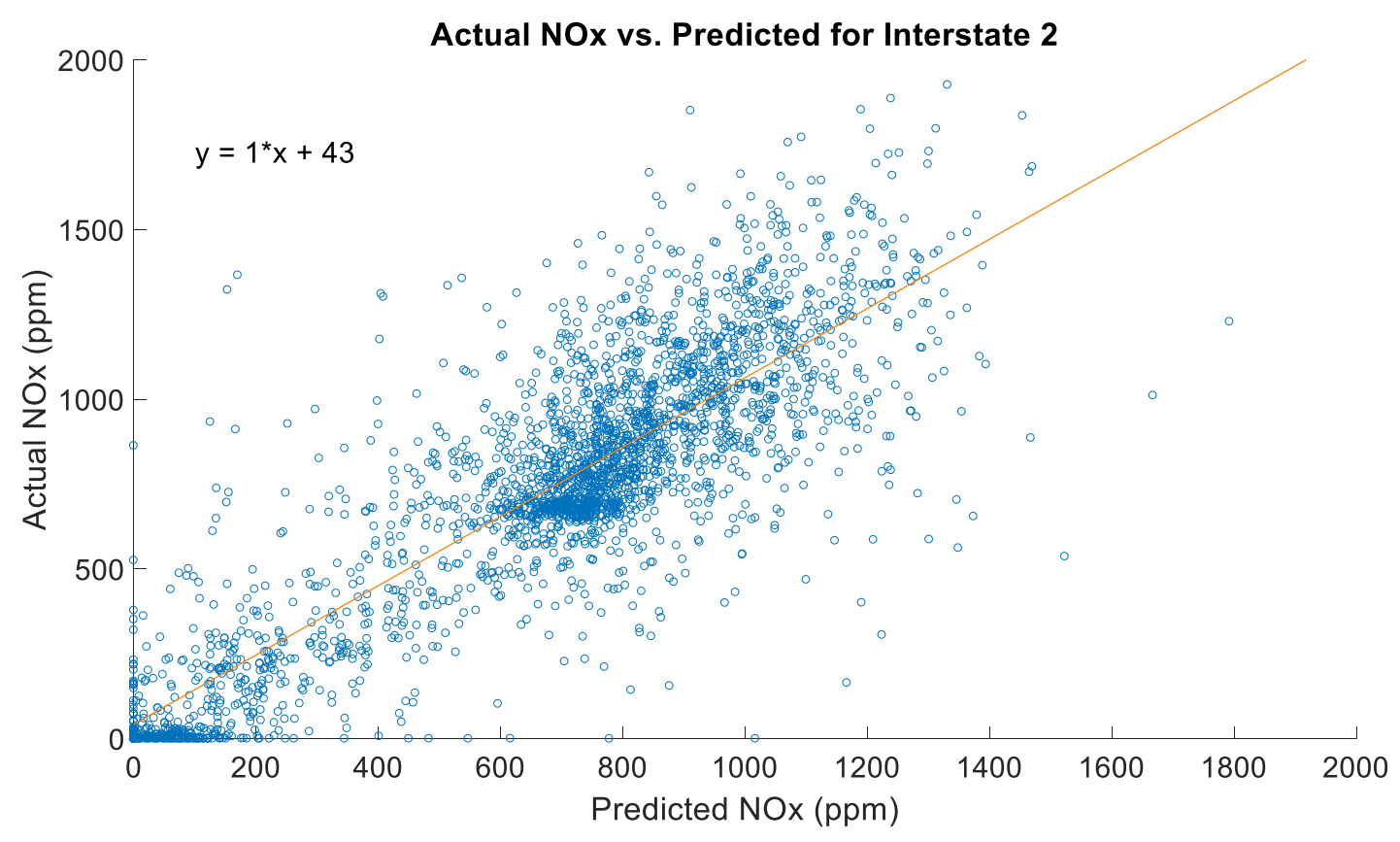

Figure 26-Scatterplot of Intersate 2 Validation Test with NN Trained with Training Data 2

Table 50-Interstate 2 Training Data 2 Correlation Values

\begin{tabular}{|c|c|}
\hline \multicolumn{2}{|c|}{ Interstate 2} \\
\hline \multicolumn{2}{|c|}{ R Value } \\
\cline { 1 - 1 } Validation Iterations & Average \\
\hline 0.84 & \multirow{2}{*}{0.82} \\
\cline { 1 - 1 } 0.79 & \\
\hline 0.81 & \\
\hline 0.84 & \\
\hline 0.83 & \\
\hline
\end{tabular}

Table 51 shows the errors in 200 second increments for the duration of the test. The first 400 seconds show mixed results between the five iterations. The first and fifth iteration show decent prediction during this time; however, the second, third, and fourth iteration have errors between 18-35 percent. The only other area of poor prediction is between $2000-2200$ seconds. 
Table 51-Interstate 2 Training Data 2-200 Second Errors

\begin{tabular}{|l|r|r|r|r|r|}
\hline \multicolumn{7}{|c|}{ Interstate 2 with Training Data Set 2 } \\
\hline & \multicolumn{1}{|c|}{ 200 Second Percent Errors } \\
\hline Time (s) & Test 1 & \multicolumn{1}{|c|}{ Test 2 } & \multicolumn{1}{c|}{ Test 3 } & \multicolumn{1}{c|}{ Test 4 } & \multicolumn{1}{c|}{ Test 5 } \\
\hline $1-201$ & 5.24 & 31.10 & 18.64 & 35.34 & 0.71 \\
\hline $201-401$ & 8.62 & 20.66 & 18.28 & 19.01 & 12.08 \\
\hline $401-601$ & 9.62 & 11.24 & 8.61 & 7.33 & 8.43 \\
\hline $601-801$ & 10.82 & 11.49 & 9.34 & 5.89 & 11.23 \\
\hline $801-1001$ & 4.61 & 5.72 & 0.34 & 1.11 & 4.45 \\
\hline $1001-1201$ & 2.02 & 2.61 & 2.89 & 0.33 & 0.53 \\
\hline $1201-1401$ & 8.01 & 3.38 & 1.66 & 7.57 & 1.48 \\
\hline $1401-1601$ & 3.34 & 5.49 & 5.51 & 7.76 & 3.50 \\
\hline $1601-1801$ & 0.50 & 3.14 & 4.75 & 1.77 & 0.08 \\
\hline $1801-2001$ & 7.99 & 8.21 & 5.39 & 5.76 & 9.13 \\
\hline $2001-2201$ & 18.06 & 18.05 & 24.74 & 15.91 & 15.55 \\
\hline $2201-2401$ & 8.89 & 7.39 & 14.42 & 6.13 & 0.79 \\
\hline $2401-2601$ & 3.04 & 12.70 & 5.57 & 1.38 & 6.35 \\
\hline
\end{tabular}

\subsection{COMPARISON OF TRAINING DATA 1 TO 2}

The average values for percent error and $\mathrm{R}$ value for each of the validation tests trained with data set one and two can be used to compare performance. Table 52 gives a good summary to how the neural network predicted values using the different training data sets. When using the highway training data set, resulted in good prediction for the highway validation tests, but a much greater error in all other validation tests. The results for prediction using training data 2 show the opposite results. Using training data 2 caused the prediction of the highway values to decrease, but improved the prediction on all other validation tests. 
Table 52-Average Percent Errors Table

\begin{tabular}{|l|r|r|}
\hline & \multicolumn{2}{|c|}{ Average Percent Error (\%) } \\
\cline { 2 - 3 } & Training Dataset 1 & Training Dataset 2 \\
\hline Highway 2 & 5.37 & 9.40 \\
\cline { 2 - 3 } Highway 3 & 0.93 & 37.24 \\
\cline { 2 - 3 } Regional & 11.25 & 6.91 \\
\cline { 2 - 3 } Local & 32.89 & 8.69 \\
\hline Neardock & 19.67 & 6.47 \\
\cline { 2 - 3 } Interstate 1 & 24.29 & 5.81 \\
\hline Interstate 2 & 20.67 & 7.84 \\
\hline
\end{tabular}

Table 53 shows the same results as Table 52 . Training data 1 provided good prediction results for the highway tests, but training data 2 improved the results of every other validation test.

Table 53-Average $R$ Values

\begin{tabular}{|l|r|r|}
\hline \multirow{2}{*}{} & \multicolumn{2}{|c|}{ Average R Value } \\
\cline { 2 - 3 } & Training Dataset 1 & Training Dataset 2 \\
\hline Highway 2 & 0.79 & 0.76 \\
\cline { 2 - 3 } Highway 3 & 0.83 & 0.56 \\
\cline { 2 - 3 } Regional & 0.69 & 0.82 \\
\cline { 2 - 3 } Local & 0.60 & 0.72 \\
\cline { 2 - 3 } Neardock & 0.63 & 0.79 \\
\cline { 2 - 3 } Interstate 1 & 0.80 & 0.90 \\
\cline { 2 - 3 } Interstate 2 & 0.69 & 0.82 \\
\hline
\end{tabular}

Table 52 and 53 shows that if highway training data is used, the neural network can predict NOx production within a few percent at highway operation, but suffers greatly for other operating conditions. Changing the training data to a mix of different locations will improve the accuracy of NOx prediction globally, but will not provide the same level of accuracy as highway prediction with highway training, with any validation tests. 


\subsection{Elimination Of LOW POWER Operation}

To explore the hypothesis that elimination of low power operation the error and $\mathrm{R}$ value were evaluated for the validation tests with and without the low power data points. Table 54 shows the comparison of the errors for each data set with and without the low power points. The validation testing with the neural network trained with Training Dataset 1 shows that the error increases in each test except for Highway 3, Local, and Interstate 1. The only test to improve the error by more than one percent was the Local data set. For the validation testing with the neural network trained with Training Dataset 2, only Highway 3 and Local showed a reduction in error. The Local test improved by less than one percent.

Table 54-Error Comparison of Low Power Removed

\begin{tabular}{|l|r|r|r|r|}
\hline \multirow{2}{*}{} & \multicolumn{4}{|c|}{ Average Percent Error (\%) } \\
\cline { 2 - 5 } & \multicolumn{1}{|c|}{ Training Dataset 1 } & \multicolumn{1}{c|}{ Training Dataset 2 } \\
\cline { 2 - 5 } & \multicolumn{1}{|c|}{ With LP } & Without LP & \multicolumn{1}{c|}{ With LP } & Without LP \\
\hline Highway 2 & 5.37 & 5.42 & 9.40 & 9.51 \\
\cline { 2 - 5 } Highway 3 & 0.93 & 0.58 & 37.24 & 30.72 \\
\cline { 2 - 5 } Regional & 11.25 & 14.57 & 6.91 & 10.10 \\
\cline { 2 - 5 } Local & 32.89 & 10.04 & 8.69 & 8.36 \\
\cline { 2 - 5 } Neardock & 19.67 & 22.24 & 6.47 & 9.82 \\
\cline { 2 - 5 } Interstate 1 & 24.29 & 23.97 & 5.81 & 8.53 \\
\cline { 2 - 5 } Interstate 2 & 20.67 & 24.61 & 7.84 & 9.51 \\
\hline
\end{tabular}

Table 55 shows the average $R$ values for the validation tests with and without the low power points. With the elimination of the low power points negatively affected the $\mathrm{R}$ value for all the data sets. 
Table 55-R Value Comparison of Low Power Removed

\begin{tabular}{|l|r|r|r|r|}
\hline \multirow{2}{*}{} & \multicolumn{4}{|c|}{ Average R Value } \\
\cline { 2 - 5 } & \multicolumn{2}{|c|}{ Training Dataset 1 } & \multicolumn{2}{c|}{ Training Dataset 2 } \\
\cline { 2 - 5 } & With LP & Without LP & \multicolumn{1}{c|}{ With LP } & Without LP \\
\hline Highway 2 & 0.79 & 0.62 & 0.76 & 0.55 \\
\cline { 2 - 5 } Highway 3 & 0.83 & 0.66 & 0.56 & 0.44 \\
\cline { 2 - 5 } Regional & 0.69 & 0.47 & 0.82 & 0.62 \\
\cline { 2 - 5 } Local & 0.60 & 0.44 & 0.72 & 0.53 \\
\cline { 2 - 5 } Neardock & 0.63 & 0.40 & 0.79 & 0.62 \\
\cline { 2 - 5 } Interstate 1 & 0.80 & 0.55 & 0.90 & 0.73 \\
\hline Interstate 2 & 0.69 & 0.56 & 0.82 & 0.66 \\
\hline
\end{tabular}

Using the information provided in Table 54 and 55, disproves the hypothesis that the neural network performs badly at low power operation. Elimination of low power operation proved to have a negative effect on the prediction results.

\subsection{FAULT INSERTED VALIDATION}

\subsubsection{NOXSENSOR FAILURE}

Using the simulated fault inserted data, the predicted NOx value could be compared to the faulty output. The following three figures display the output of the neural network prediction compared to the NOx sensor output. Figure 27 shows the step failure of the NOx sensor. It can be seen that beginning around 2700 seconds, the NOx sensor shifts upwards, and has separation from the readings to the prediction. 

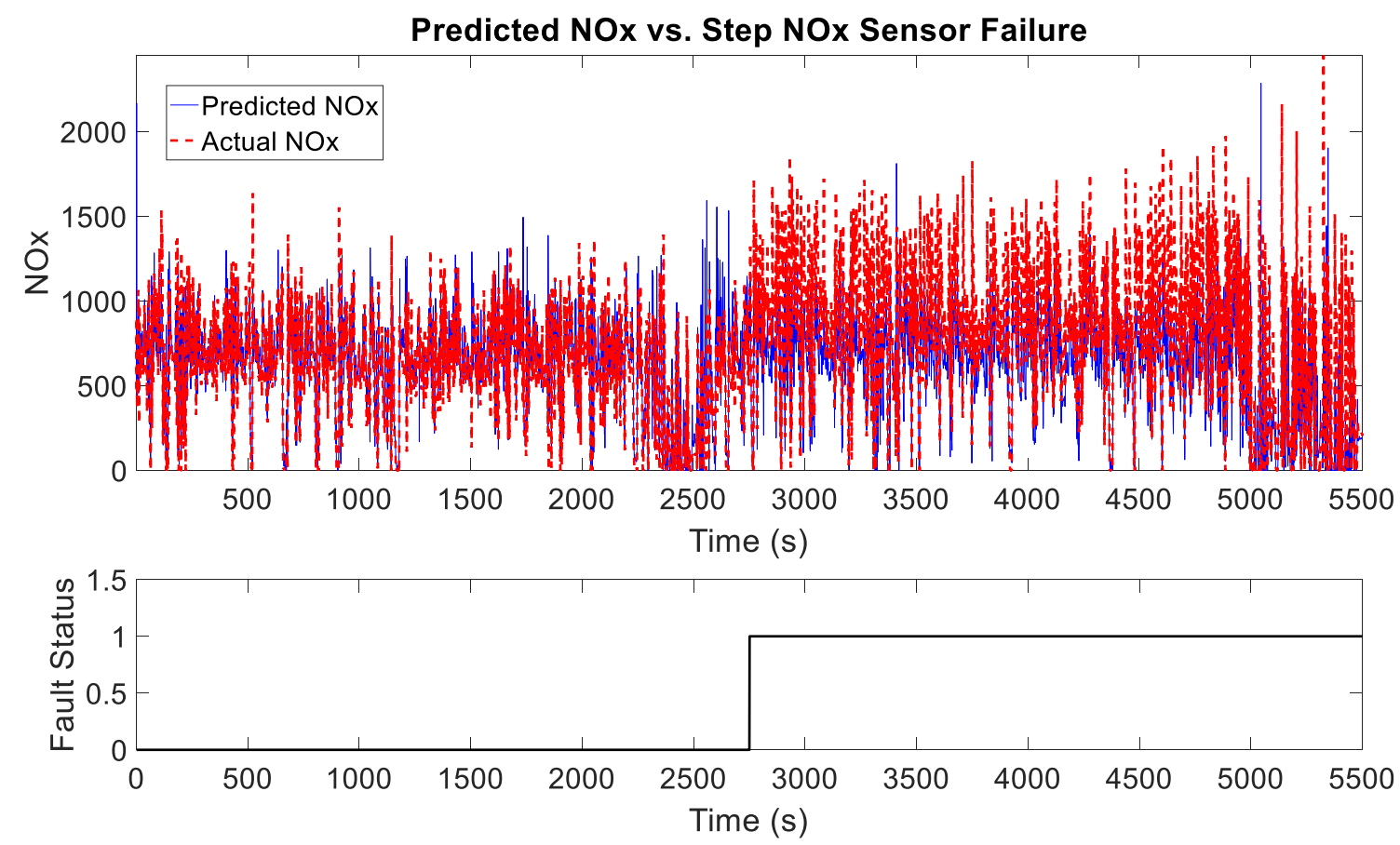

Figure 27-Step NOx Sensor Failure

Figure 28 shows the prediction compared to the NOx sensor reading with a ramp failure. The ramp failure becomes evident around 4250 seconds, where the sensor values are continually drifting further from the predicted values.
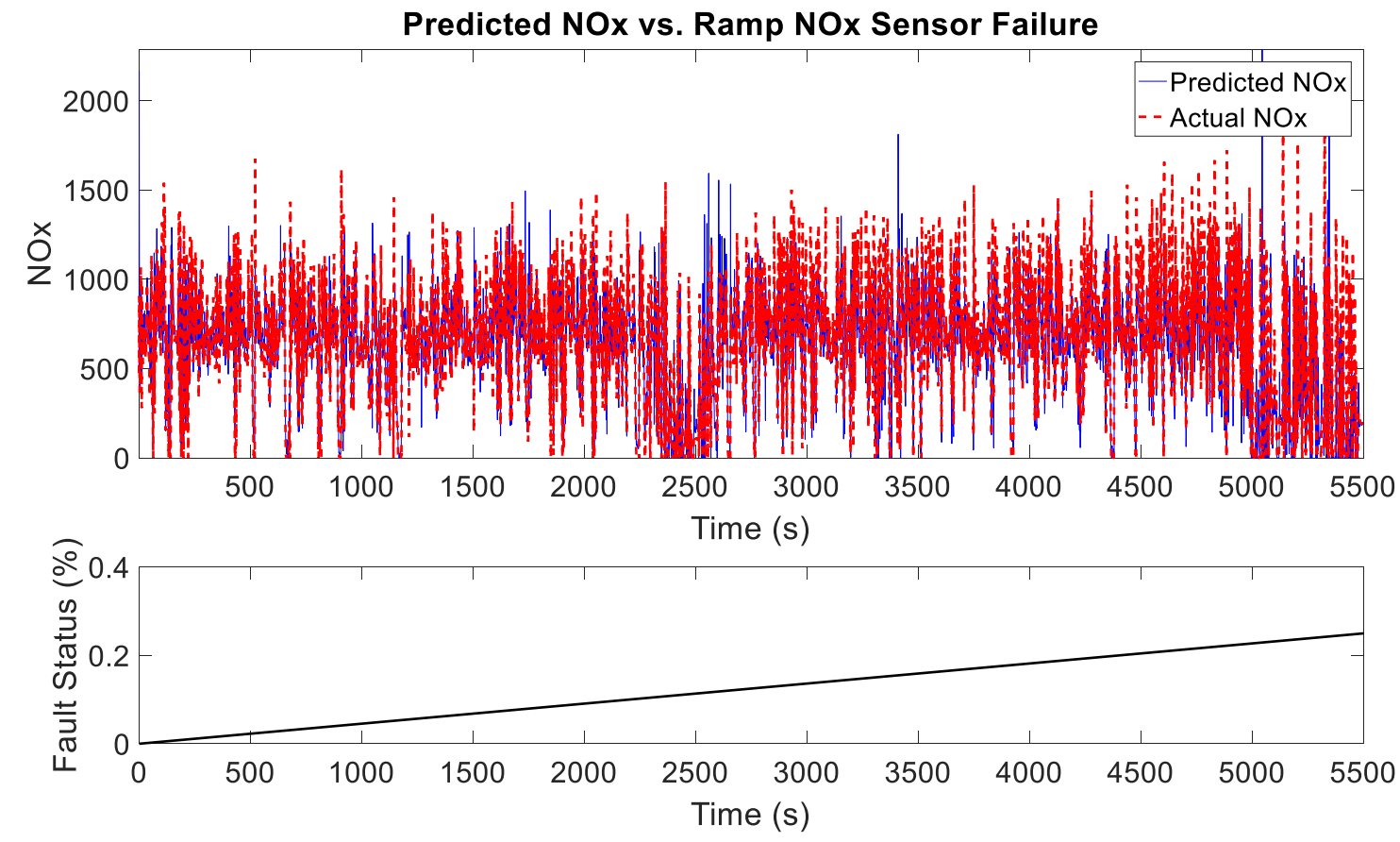

Figure 28-Ramp NOx Sensor Failure 
Figure 29 shows the prediction versus the NOx sensor readings with a square wave failure. This graph shows obvious separation every 400 seconds where the sensor reading goes to zero.
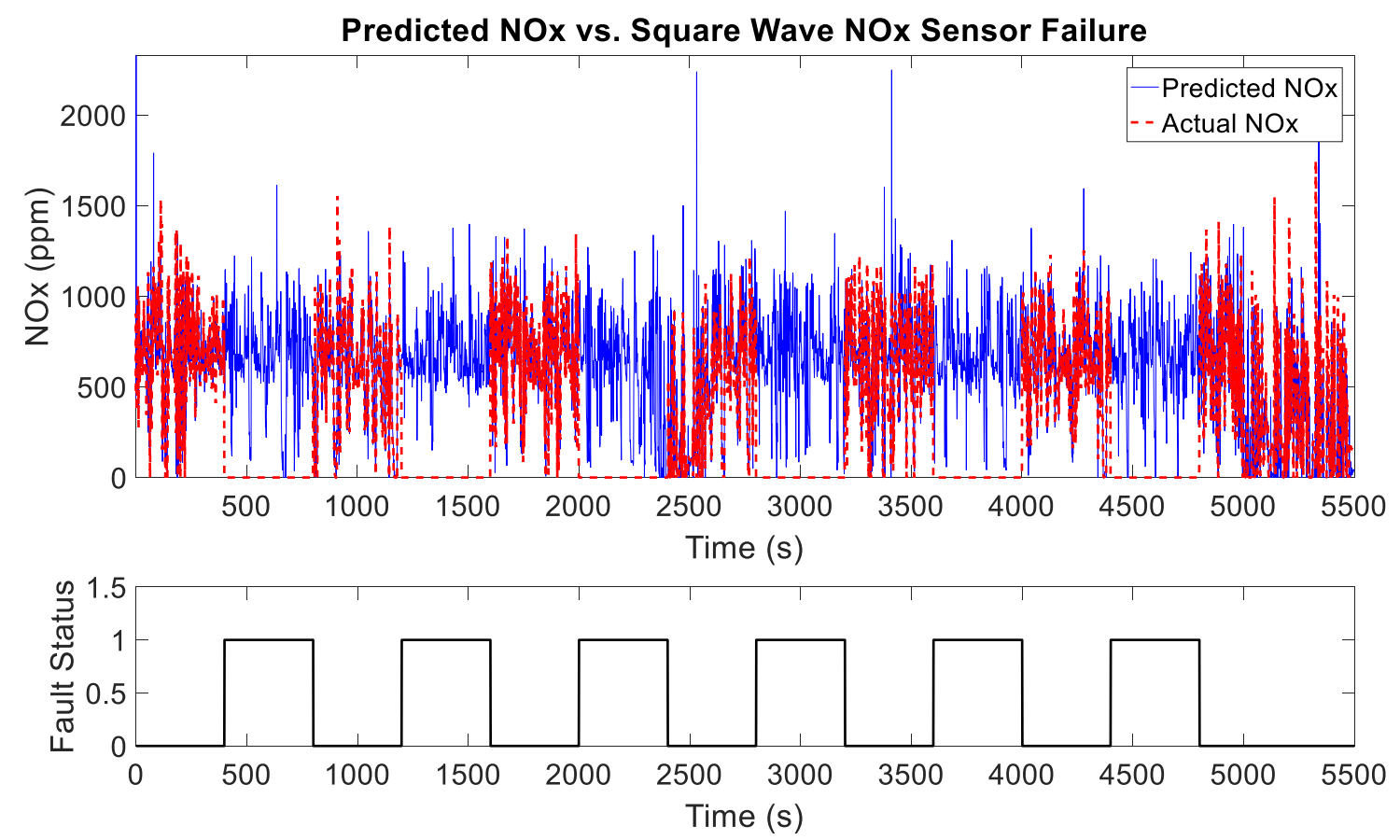

Figure 29-Square Wave NOx Sensor Failure

The percent errors between the neural network prediction and sensor reading can be seen in Table 56. This table displays the errors in 200 second increments for evaluation. For the step failure data set, the highlighted regions show when the fault occurs how the error is affected. For the ramp failure method, the increase in error is evident, but not as large as the step failure. The square function failure produced the largest change in error between the predicted and actual values with some error values changing by nearly 200 percent. This amount of change would make it very easy to determine when there was a problem. 
Table 56-NOx Sensor Fault Errors

\begin{tabular}{|c|c|c|c|c|c|c|c|}
\hline \multirow[b]{2}{*}{ Time (s) } & \multirow[b]{2}{*}{$\begin{array}{l}\text { Nominal } \\
\text { NOx } \\
\text { Reading }\end{array}$} & \multicolumn{2}{|c|}{ Step Failure } & \multicolumn{2}{|c|}{ Ramp Failure } & \multicolumn{2}{|c|}{ Square Function Failure } \\
\hline & & $\begin{array}{l}\text { Fault } \\
\text { Status }\end{array}$ & NOx Value & $\begin{array}{c}\text { Fault } \\
\text { Status } \\
(\%)\end{array}$ & NOx value & $\begin{array}{l}\text { Fault } \\
\text { Status }\end{array}$ & NOx Value \\
\hline $1-201$ & 0.80 & 0 & 0.32 & 1.00 & 0.76 & 0 & 0.32 \\
\hline $201-401$ & 1.38 & 0 & 1.19 & 1.01 & 2.54 & 0 & 0.05 \\
\hline $401-601$ & 1.22 & 0 & 0.46 & 1.02 & 1.77 & 1 & 200.00 \\
\hline $601-801$ & 2.75 & 0 & 0.52 & 1.03 & 2.61 & 1 & 198.14 \\
\hline $801-1001$ & 1.18 & 0 & 4.18 & 1.04 & 0.16 & 0 & 4.18 \\
\hline $1001-1201$ & 4.35 & 0 & 6.88 & 1.05 & 2.04 & 0 & 8.01 \\
\hline $1201-1401$ & 0.97 & 0 & 1.74 & 1.06 & 4.01 & 1 & 200.00 \\
\hline $1401-1601$ & 3.91 & 0 & 4.53 & 1.07 & 2.06 & 1 & 197.58 \\
\hline $1601-1801$ & 2.38 & 0 & 0.35 & 1.08 & 7.78 & 0 & 0.35 \\
\hline $1801-2001$ & 3.45 & 0 & 3.65 & 1.09 & 4.63 & 0 & 4.91 \\
\hline $2001-2201$ & 1.43 & 0 & 0.84 & 1.10 & 8.27 & 1 & 200.00 \\
\hline $2201-2401$ & 4.24 & 0 & 4.56 & 1.11 & 5.32 & 1 & 200.00 \\
\hline $2401-2601$ & 7.65 & 0 & 4.71 & 1.12 & 6.15 & 0 & 4.71 \\
\hline $2601-2801$ & 15.44 & 0 & 8.67 & 1.13 & 6.91 & 0 & 19.80 \\
\hline $2801-3001$ & 2.48 & 1 & 35.02 & 1.14 & 14.09 & 1 & 200.00 \\
\hline $3001-3201$ & 0.84 & 1 & 29.22 & 1.15 & 8.93 & 1 & 197.75 \\
\hline $3201-3401$ & 1.08 & 1 & 34.56 & 1.16 & 15.16 & 0 & 1.26 \\
\hline $3401-3601$ & 6.98 & 1 & 19.87 & 1.17 & 1.05 & 0 & 14.77 \\
\hline $3601-3801$ & 1.62 & 1 & 29.38 & 1.18 & 11.45 & 1 & 200.00 \\
\hline $3801-4001$ & 3.16 & 1 & 34.53 & 1.19 & 17.50 & 1 & 197.74 \\
\hline $4001-4201$ & 0.89 & 1 & 30.52 & 1.20 & 14.16 & 0 & 2.89 \\
\hline $4201-4401$ & 9.82 & 1 & 22.60 & 1.21 & 6.87 & 0 & 12.25 \\
\hline $4401-4601$ & 0.59 & 1 & 31.03 & 1.22 & 16.20 & 1 & 200.00 \\
\hline $4601-4801$ & 3.99 & 1 & 37.40 & 1.23 & 23.43 & 1 & 199.27 \\
\hline $4801-5001$ & 1.50 & 1 & 31.12 & 1.24 & 17.74 & 0 & 2.28 \\
\hline
\end{tabular}

\subsubsection{Boost Pressure Sensor FaILURe}

Figures 30, 31, and 32 show how different boost pressure sensor failures would affect the prediction compared to the NOx sensor reading. Figure 30 shows a step failure of the boost pressure sensor, which causes the prediction to shift upwards away from the actual measured value. 

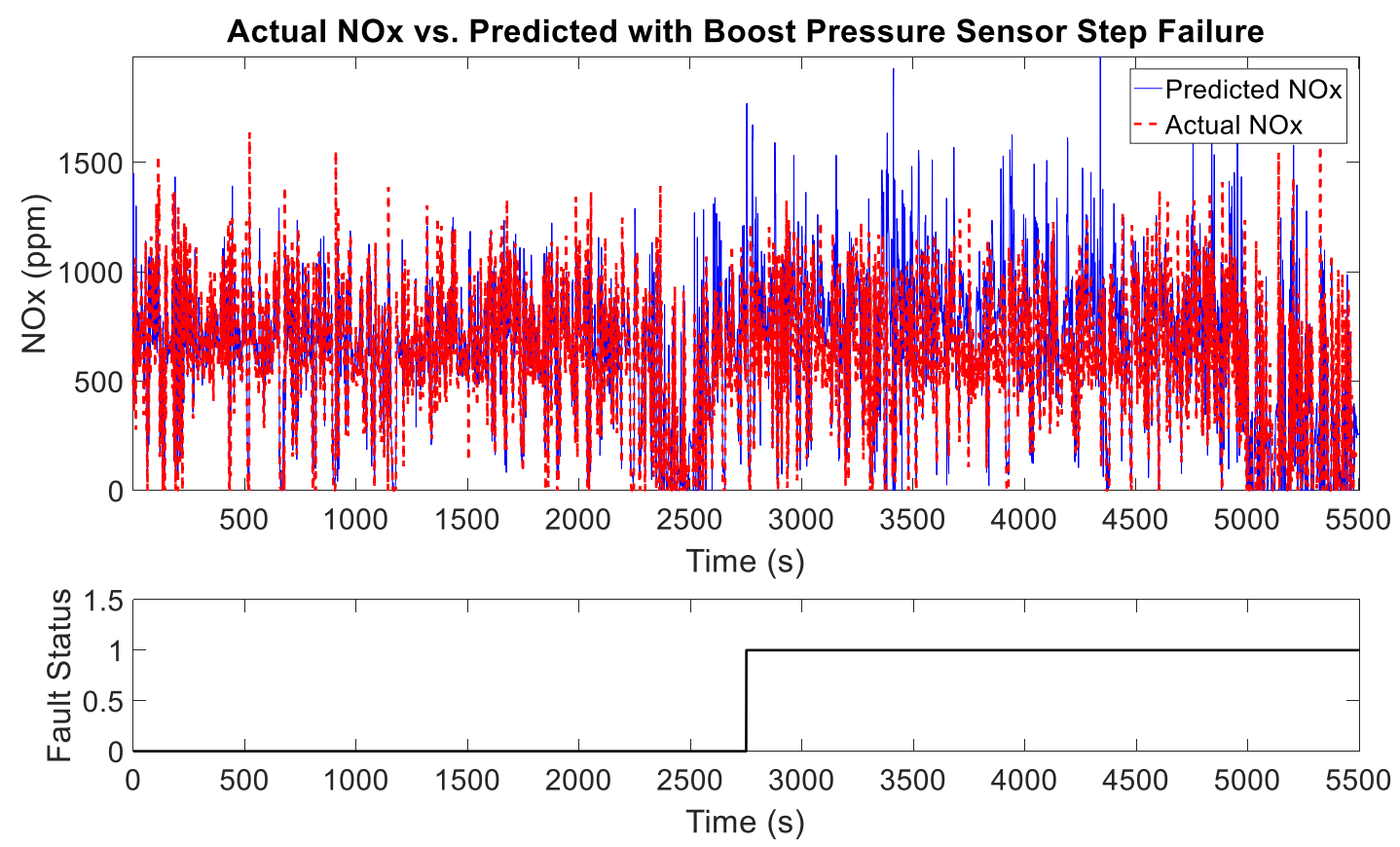

Figure 30-Boost Pressure Sensor Step Failure

Figure 31 displays how the prediction would be affected with a ramp failure of the boost pressure sensor. The shift in prediction begins to become visible around 2600 seconds, and can be seen for the rest of the test.
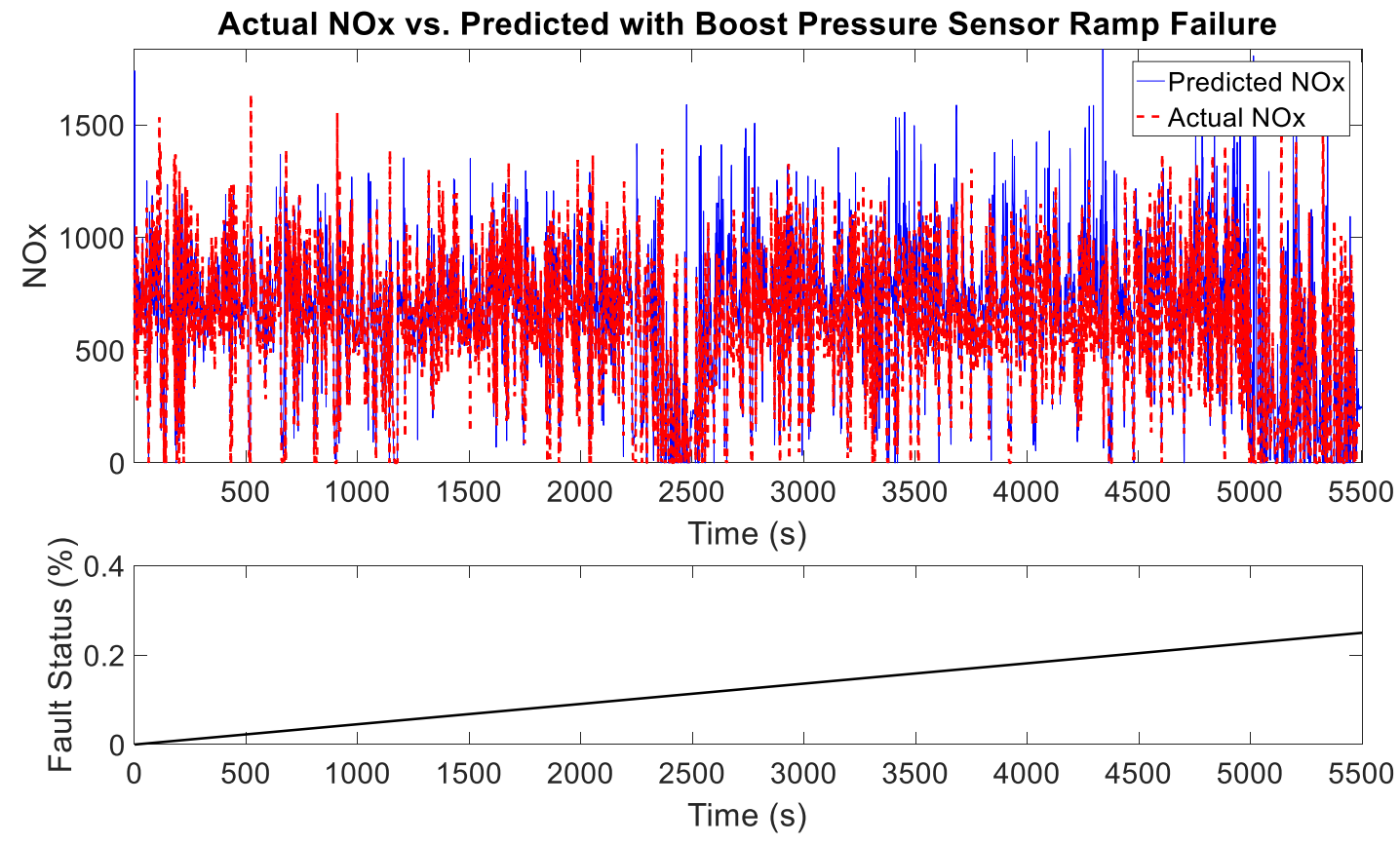

Figure 31-Boost Pressure Sensor Ramp Failure 
Figure 32 shows how the NOx prediction is affected when the boost pressure sensor has a square function failure. The prediction is shifted upwards in the sections where the boost pressure sensor is input as zero.
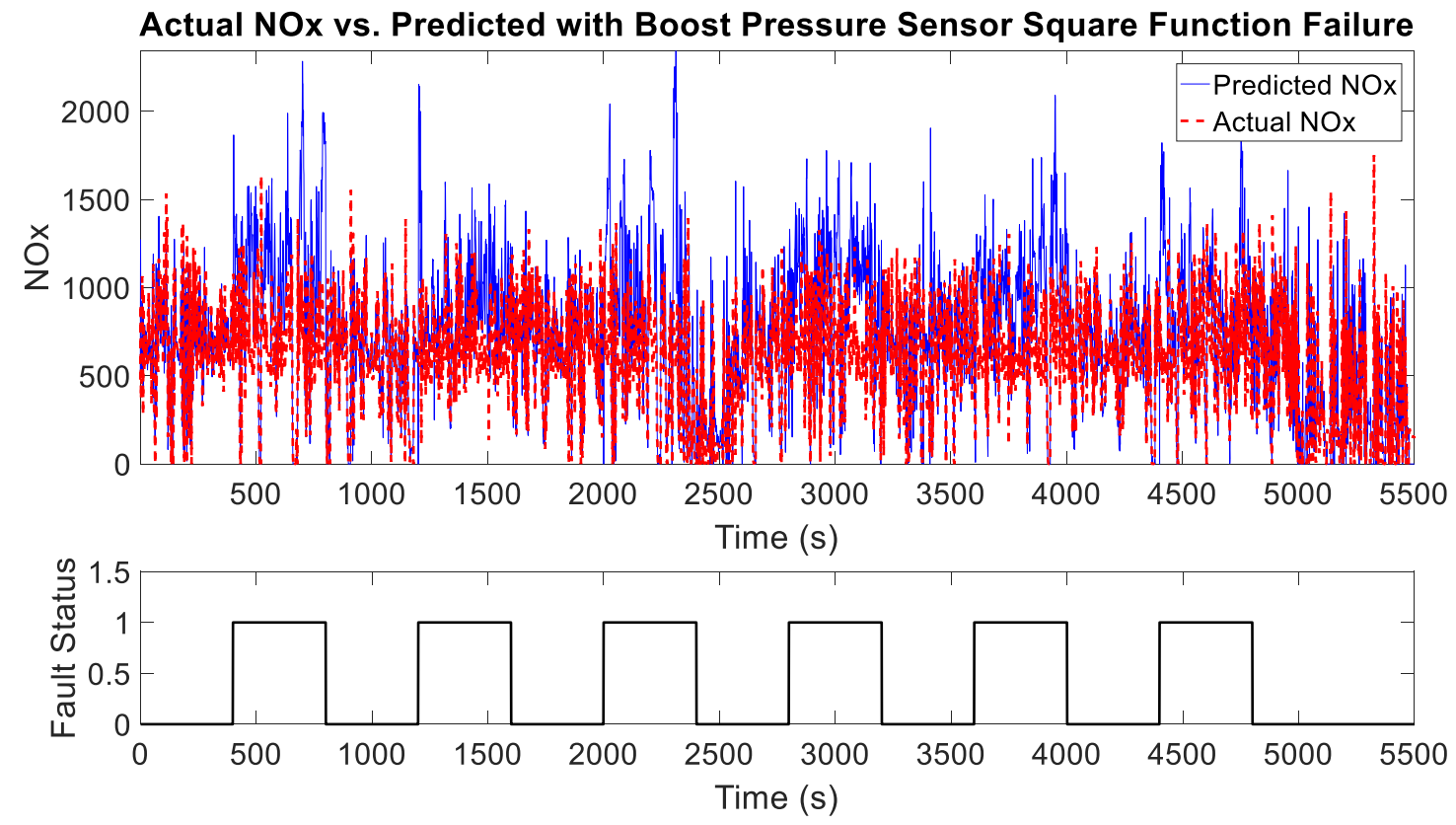

Figure 32-Boost Pressure Sensor Square Function Failure

The errors in 200 second increments can be seen in Table 57. In column two, the highlighted values show the increase in error once the step failure has occurred. Similarly, in column three the highlighted values show where the ramp failure is enough to cause a shift in error. The forth column of the table shows the segments where the square function failure has occurred and the sizeable shift in error that results. 
Table 57-Boost Pressure Sensor Failure Errors

\begin{tabular}{|c|c|c|c|c|c|c|c|}
\hline \multirow{2}{*}{ Time (s) } & \multirow[b]{2}{*}{$\begin{array}{l}\text { Nominal } \\
\text { NOx } \\
\text { Reading }\end{array}$} & \multicolumn{2}{|c|}{ Step Failure } & \multicolumn{2}{|c|}{ Ramp Failure } & \multicolumn{2}{|c|}{ Square Function Failure } \\
\hline & & $\begin{array}{c}\text { Fault } \\
\text { Status }\end{array}$ & NOx Value & $\begin{array}{c}\text { Fault } \\
\text { Status } \\
(\%) \\
\end{array}$ & NOx value & \begin{tabular}{|c} 
Fault \\
Status \\
\end{tabular} & NOx Value \\
\hline $201-401$ & 1.38 & 0 & 1.25 & 1.01 & 1.45 & 0 & 0.53 \\
\hline $401-601$ & 1.22 & 0 & 1.10 & 1.02 & 0.36 & 1 & 39.24 \\
\hline $601-801$ & 2.75 & 0 & 0.77 & 1.03 & 3.66 & 1 & 47.11 \\
\hline $801-1001$ & 1.18 & 0 & 2.64 & 1.04 & 4.93 & 0 & 4.51 \\
\hline $1001-1201$ & 4.35 & 0 & 2.63 & 1.05 & 5.78 & 0 & 5.83 \\
\hline $1201-1401$ & 0.97 & 0 & 0.24 & 1.06 & 1.68 & 1 & 21.91 \\
\hline $1401-1601$ & 3.91 & 0 & 4.66 & 1.07 & 4.99 & 1 & 34.03 \\
\hline $1601-1801$ & 2.38 & 0 & 1.97 & 1.08 & 0.64 & 0 & 2.37 \\
\hline $1801-2001$ & 3.45 & 0 & 3.23 & 1.09 & 5.12 & 0 & 3.30 \\
\hline $2001-2201$ & 1.43 & 0 & 0.54 & 1.10 & 3.38 & 1 & 35.24 \\
\hline $2201-2401$ & 4.24 & 0 & 3.75 & 1.11 & 9.13 & 1 & 47.74 \\
\hline $2401-2601$ & 7.65 & 0 & 5.71 & 1.12 & 15.29 & 0 & 27.77 \\
\hline $2601-2801$ & 15.44 & 0 & 15.86 & 1.13 & 23.21 & 0 & 14.91 \\
\hline $2801-3001$ & 2.48 & 1 & 3.18 & 1.14 & 2.29 & 1 & 28.56 \\
\hline $3001-3201$ & 0.84 & 1 & 18.39 & 1.15 & 9.13 & 1 & 41.19 \\
\hline $3201-3401$ & 1.08 & 1 & 6.50 & 1.16 & 7.53 & 0 & 1.92 \\
\hline $3401-3601$ & 6.98 & 1 & 20.49 & 1.17 & 18.59 & 0 & 11.42 \\
\hline $3601-3801$ & 1.62 & 1 & 17.53 & 1.18 & 10.12 & 1 & 31.60 \\
\hline $3801-4001$ & 3.16 & 1 & 10.32 & 1.19 & 9.76 & 1 & 38.79 \\
\hline $4001-4201$ & 0.89 & 1 & 8.58 & 1.20 & 11.64 & 0 & 1.10 \\
\hline $4201-4401$ & 9.82 & 1 & 23.33 & 1.21 & 20.94 & 0 & 7.51 \\
\hline $4401-4601$ & 0.59 & 1 & 13.76 & 1.22 & 9.04 & 1 & 28.72 \\
\hline $4601-4801$ & 3.99 & 1 & 8.46 & 1.23 & 5.14 & 1 & 16.35 \\
\hline $4801-5001$ & 1.50 & 1 & 8.21 & 1.24 & 10.98 & 0 & 1.49 \\
\hline
\end{tabular}




\section{CONCLUSIONS}

This thesis investigated the use of an artificial neural network to be used for the modeling of engine out NOx for maintenance and OBD software. From the results two different helpful pathways can be exploited. The first pathway is that if the ANN is trained using highway data, the prediction during highway use is accurate within 1-5\%. This makes it possible for an OBD monitor to be created using highway enabled conditions. If enable conditions are set so that the monitor only runs during highway use, NOx production can be predicted accurately enough to detect faults in the NOx sensor and other engine subsystems. The second pathway is that the combined training data can be used for NOx prediction at all operating states to loosely monitor the production of NOx emissions.

Using this neural network trained with highway data, it was shown that detection of failing sensors is possible. Step, ramp, and square function failures of the NOx sensor and boost pressure sensor cause changes in the error from 10-200\% between the actual and predicted values when the fault was inserted.

This study shows that a neural network is an efficient way to provide an output to an algorithm to identify faults of the NOx sensor, and various other engine sensors. With further development of the accuracy of the NOx prediction, detection of fault below the ever decreasing fault limits would be possible in real time. 


\section{LIMITATIONS}

A model of this nature has the capability to include any number of parameter for evaluation. There are some variables that affect NOx production which were not considered in this study. For example, ambient air humidity was not used as an input, or as a filter when gathering validation data. It was found that the ambient humidity for all the data was between $40 \%-60 \%$, except for the two interstate data sets. The two interstate data sets had an average ambient humidity of $10 \%$, which may account for the differentiation in NOx between the highway and interstate data sets. Another ambient condition that could affect the performance of the neural network is the ambient temperature. The ambient temperature was between $12-30^{\circ} \mathrm{C}$ for the data. Additional testing could lead to a correction factor applied to the NOx prediction for more accurate results. One additional limitation could be that all of the data used for the neural network was solely based on engine parameters. An investigation into vehicle parameters such as vehicle speed could be beneficial to the accuracy of the prediction. 


\section{RECOMMENDATIONS}

To further develop the accuracy and usability of this artificial neural network method, two additional changes can be made.

The first recommendation I would make would be to implement the use of manufacture parameters such as EGR, SOI, needle opening pressure (NOP), and VGT position. These parameters would not only allow for a better understanding of the exact operating state of the engine, but also a direct line of monitoring for the condition of the engine subsystems.

The second recommendation is to develop a matrix of neural networks to be used for NOx prediction rather than using one network to predict NOx production at all operating states. This study showed that when the neural network was trained with a dataset similar to that which the engine is operating in, NOx can be predicted to a high level of accuracy. The solution to this is to utilize a matrix of neural networks which categorizes incoming data by power level and vehicle speed. In each category, a neural network can be trained using training data (TD) that directly corresponds to that category. A matrix of the proposed idea can be seen in Table 58 .

Table 58-Vehicle Speed vs. Power Matrix

\begin{tabular}{|c|c|c|c|c|}
\hline & \multicolumn{4}{|c|}{ Vehicle Speed } \\
\hline Power & $<1 \mathrm{mph}$ & $1-25 \mathrm{mph}$ & $25-50 \mathrm{mph}$ & $>50 \mathrm{mph}$ \\
\hline$<25 \%$ & TD1 & TD2 & TD3 & TD4 \\
\hline $25-50 \%$ & TD5 & TD6 & TD7 & TD8 \\
\hline$>50 \%$ & TD9 & TD10 & TD11 & TD12 \\
\hline
\end{tabular}


To further develop the software to not only detect a fault in the engine or NOx sensor, a diagnostics software must be used. There are two possibilities that would work with the neural network output. One method would include a k-means clustering algorithm. This algorithm would use the output of the predicted vs actual NOx and label the clusters of points when a fault is present, depending on the distance and location from the nominal correspondence line. A second method would be to use a "drop out" method. This drop out method could implement software coding that would remove inputs to the ANN one by one and evaluate the prediction compared to that with all inputs present. The ANN will have better prediction with one of the 12 inputs eliminated compared to all 12 inputs with one being faulty, so by iterating which input parameter is removed, the faulty sensor can be identified when that input is removed and the prediction returns to predicting measured values. 


\section{REFERENCES}

1. COMAR 26.11.34.02: California Code of Regulations (CCR), title 13 motor vehicles, division 3 air resources board. Annapolis, MD: Office of the Secretary, Div. of State Documents, 2013.

2. Code of Federal regulations, Title 40 Part 86. Washington: Office of the Federal Register, National Archives and Records Service, General Services Administration.

3. Merrion, D., "Heavy Duty Diesel Emission Regulations - Past, Present, and Future," SAE Technical Paper 2003-01-0040, 2003, doi:10.4271/2003-01-0040.

4. "Carbon Monoxide Poisoning." Centers for Disease Control and Prevention. December 30, 2015. Accessed April 2, 2017. https://www.cdc.gov/co/faqs.htm.

5. Control, Division Of Air Pollution. "How Smog Affects Health." EPA IMAGE. Accessed April 02, 2017. http://epa.ohio.gov/dapc/echeck/whyecheck/healthef.aspx.

6. Pulkrabek, W. W. Engineering Fundamentals of the Internal Combustion Engine. Unid States of America: Prentice Hall, 2004.

7. "Progress Cleaning the Air and Improving People's Health." EPA. February 16, 2017. Accessed April 12, 2017. https://www.epa.gov/clean-air-act-overview/progress-cleaningair-and-improving-peoples-health.

8. "On-Board Diagnostic (OBD) Regulations and Requirements: Questions and Answers ." $\begin{array}{llll}\text { EPA. Accessed } & \text { April } & 017 .\end{array}$ https://nepis.epa.gov/Exe/ZyPURL.cgi?Dockey=P100LW9G.TXT.

9. Zentiz Follow. "On-Board Diagnostics (OBD) Program Overview." LinkedIn SlideShare. July 11, 2016. Accessed April 05, 2017. http://www.slideshare.net/tonnytran29/onboarddiagnostics-obd-program-overview.

10. Shanmuganathan, S., and Samarasinghe, S. Artificial Neural Network Modelling. Cham: Springer, 2016.

11. Perhinschi, M., Artificial Intelligence Techniques in Engineering. Ch. 4. Handout 9. Morgantown: West Virginia University, 2016.

12. Perhinschi, M., Artificial Intelligence Techniques in Engineering. Ch. 4. Handout 10. Morgantown: West Virginia University, 2016. 
13. Gadallah, A., Elshenawy, E., Elzahaby, A., El-Salmawy, H., and Bawady, A. "Application of Neural Networks for Prediction and Optimization of Emissions and Performance in a Hydrogen Fuelled Direct Injection Engine Equipped With In Cylinder Water Injection." SAE Technical Paper Series, 2009. doi:10.4271/2009-01-2684.

14. Singhsandhu, G., and Salaria, D. "A Bayesian Network Model of the Particle Swarm Optimization for Software Effort Estimation." International Journal of Computer Applications 96, no. 4 (2014): 52-58. doi:10.5120/16786-6371.

15. Ham, F., and Kostanic, I. Principles of Neurocomputing for Science and Engineering. Boston: McGraw Hill, 2001.

16. Li, H., Butts, K., Zaseck, K., Liao-McPherson, D., Kolmanovsky, I. "Emissions Modeling of a Light-Duty Diesel Engine for Model-Based Control Design Using Multi-Layer Perceptron Neural Networks," SAE Technical Paper 2017-01-0601, 2017, doi:10.4271/2017-01-0601.

17. Anand, G., Gopinath, S., Ravi, M., Kar, I., Subrahmanyam, J. "Artificial Neural Networks for Prediction of Efficiency and NOx Emission of a Spark Ignition Engine," SAE Technical Paper 2006-01-1113, 2006, doi:10.4271/2006-01-1113.

18. Zhang, Q., Pennycott, A., Burke, R., Akehurst, S., Brace, C. "Predicting the Nitrogen Oxides Emissions of a Diesel Engine using Neural Networks," SAE Technical Paper 2015-01-1626, 2015, doi:10.4271/2015-01-1626.

19. Krijnsen, H. C., Van Kooten1, W. E. J., Calis, H. P. A., Verbeek, R. P. and Van Den Bleek, C. M. (2000), Evaluation of an artificial neural network for NOX emission prediction from a transient diesel engine as a base for NOX control. Can. J. Chem. Eng., 78: 408-417. doi:10.1002/cjce.5450780218

20. Freund, Rudolf J., Wilson, W., and Mohr, D. Statistical Methods. Amsterdam: Elsevier, 2010.

21. Orban, J., Naber, S., Sharp, C., Khair, M., McGillSentech, R. "Long-Term Aging of NOx Sensors in Heavy-Duty Engine Exhaust," SAE Technical Paper 2005-01-3793, 2005, doi:10.4271/2005-01-3793.

22. Orlando, I., MAJOR, F. Greitzer, L. Kangas, and T. Reeve. "AN ARTIFICIAL NEURAL NETWORK SYSTEM FOR DIAGNOSING GAS TURBINE ENGINE FUEL FAULT." February 10, 1994. 
23. Evans, James D. Straightforward statistics for the behavioral sciences. Pacific Grove: Brooks/Cole Publishing Company, 1996. 


\section{APPENDICES}

Appendix A-F include the Matlab code that was used to collect, organize, and normalize the ECU data. It also includes the Matlab code used for the training, testing, and prediction of the neural network. Lastly it includes the plotting and data evaluation code.

\subsection{APPENDIX A}

Appendix A shows the Highway 1 Preprocessing code. The purpose of this code was to collect, normalize, and format the desired inputs into a matrix.

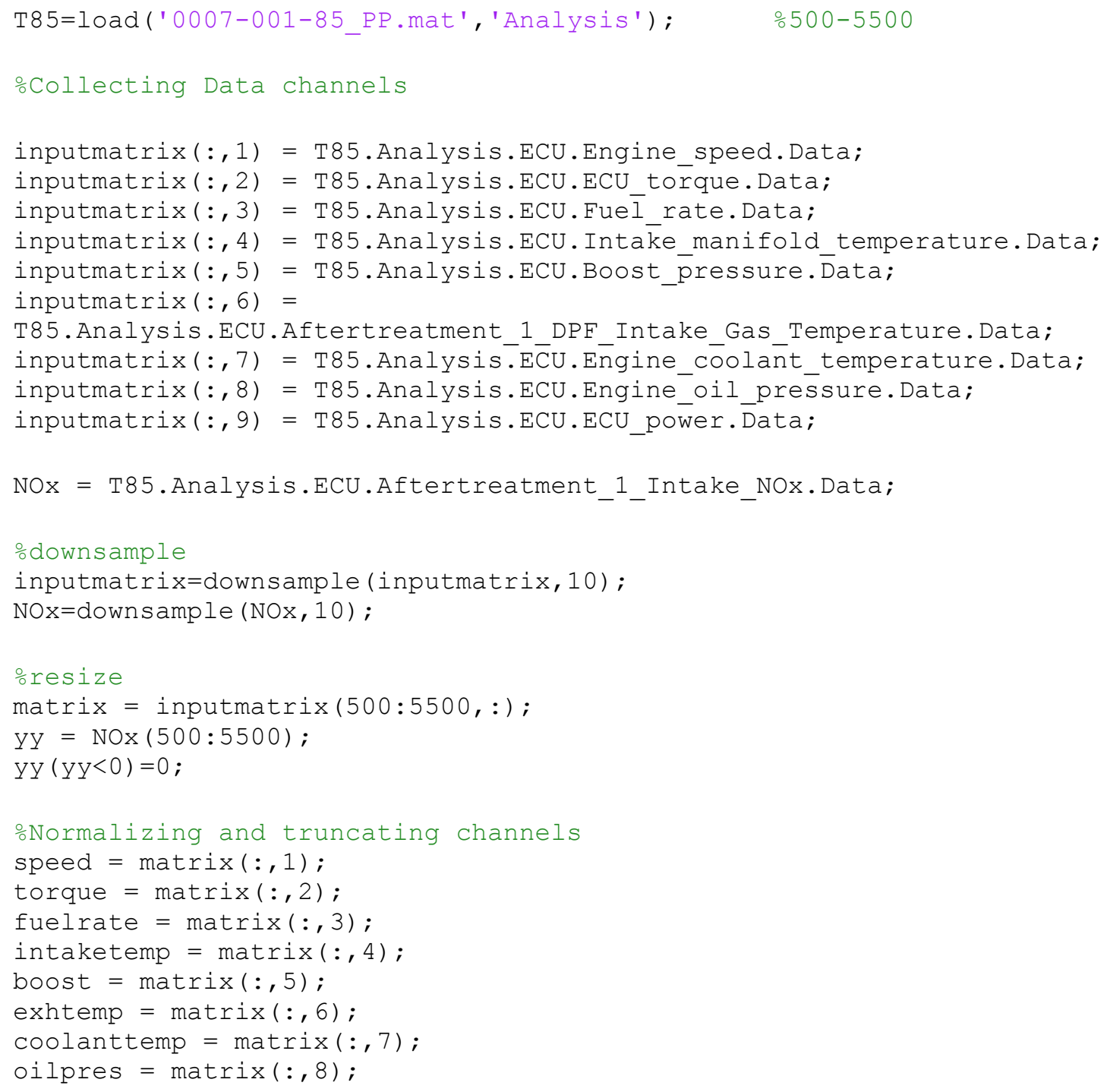




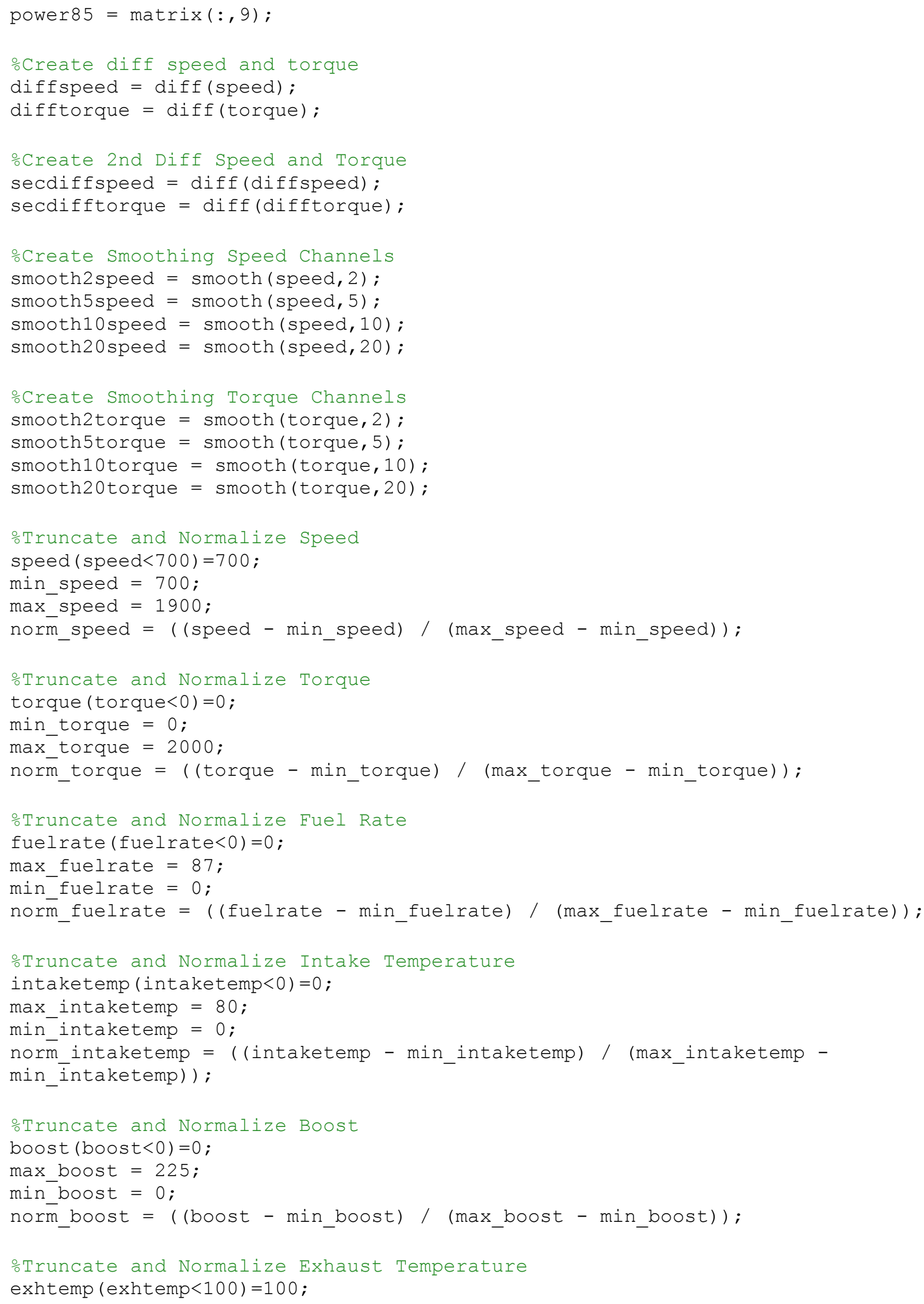




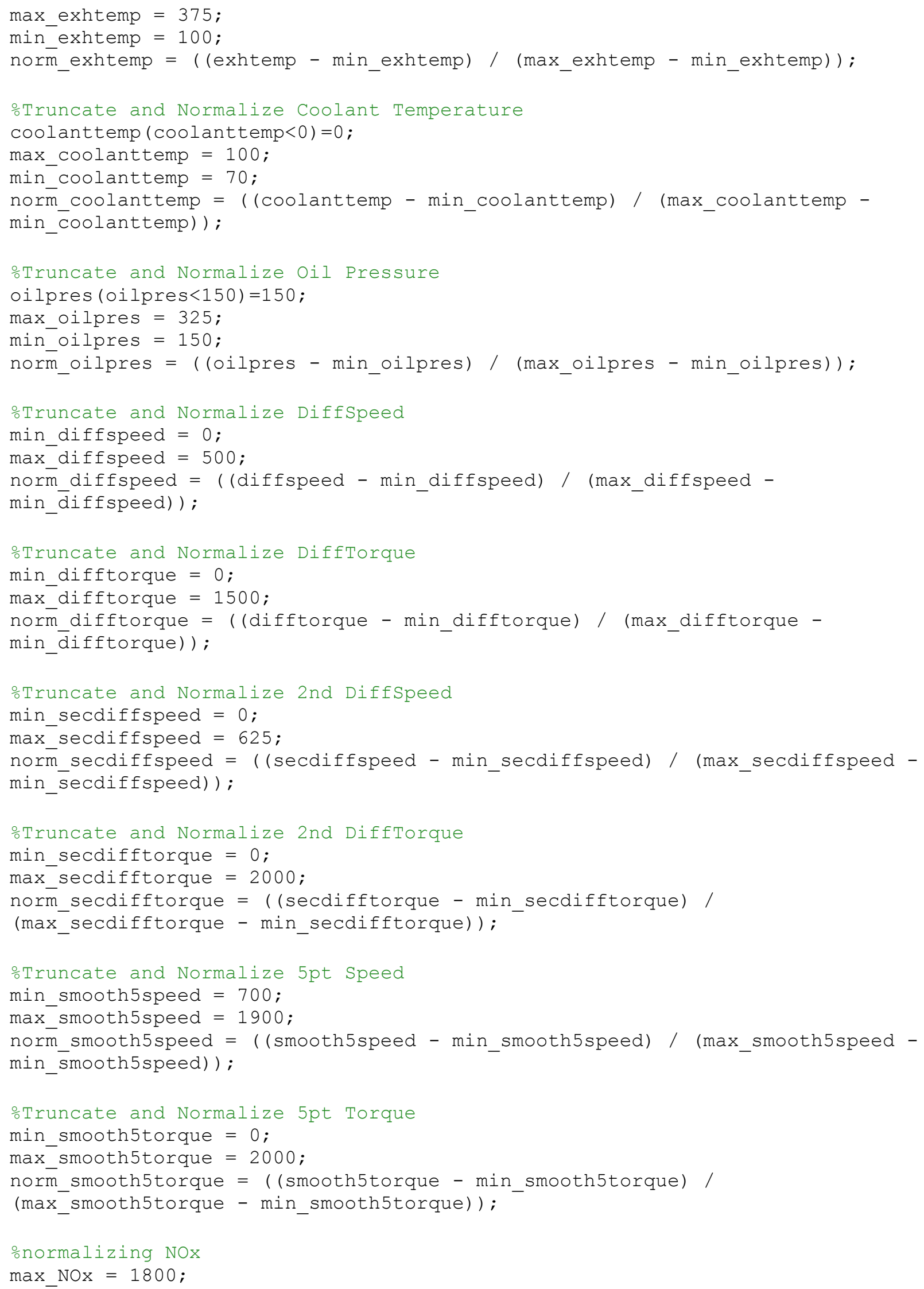




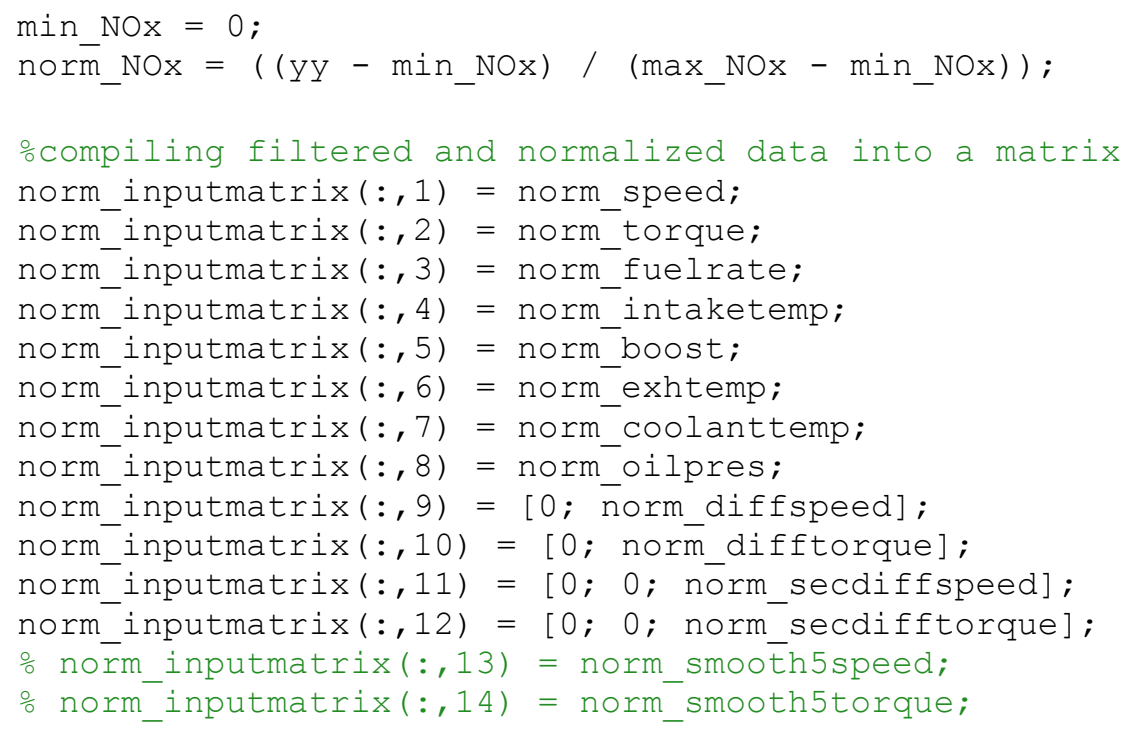

\subsection{APPENDIX B}

Appendix B shows the Combined Training Data code. The purpose of this code was to collect the four desired portions of ECU data to be used within the combine data set. The data was normalized and assembled into a matrix.

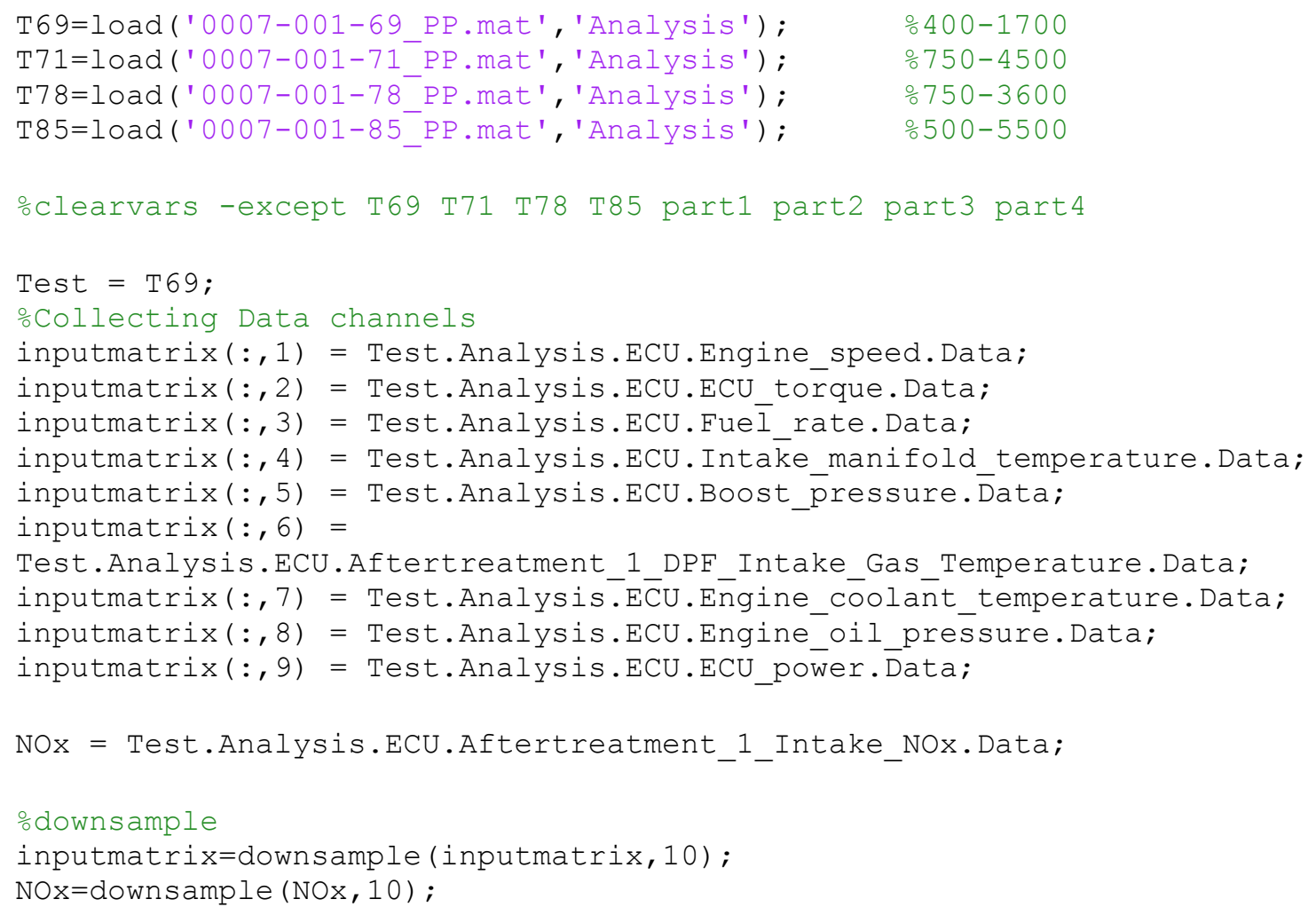




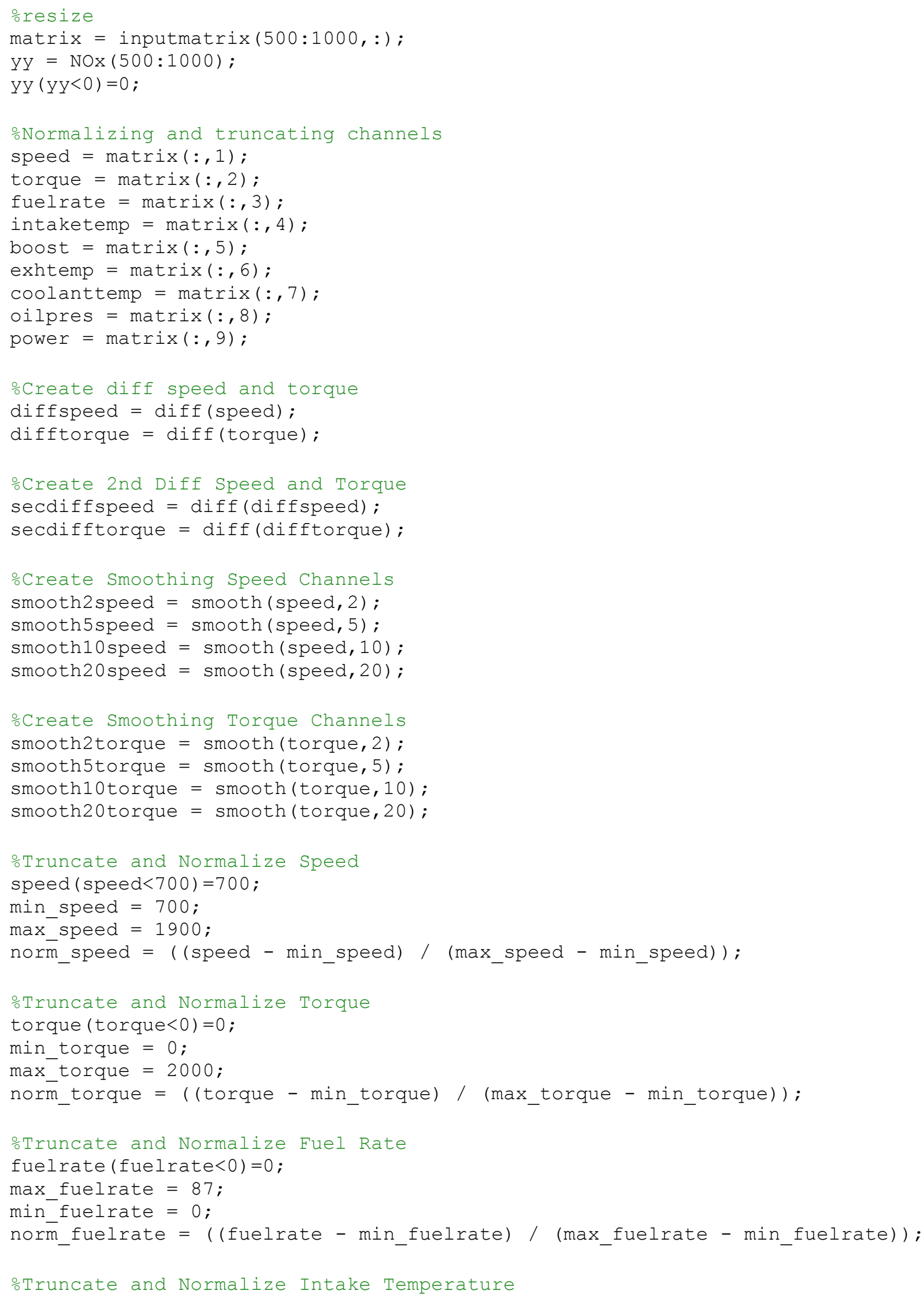




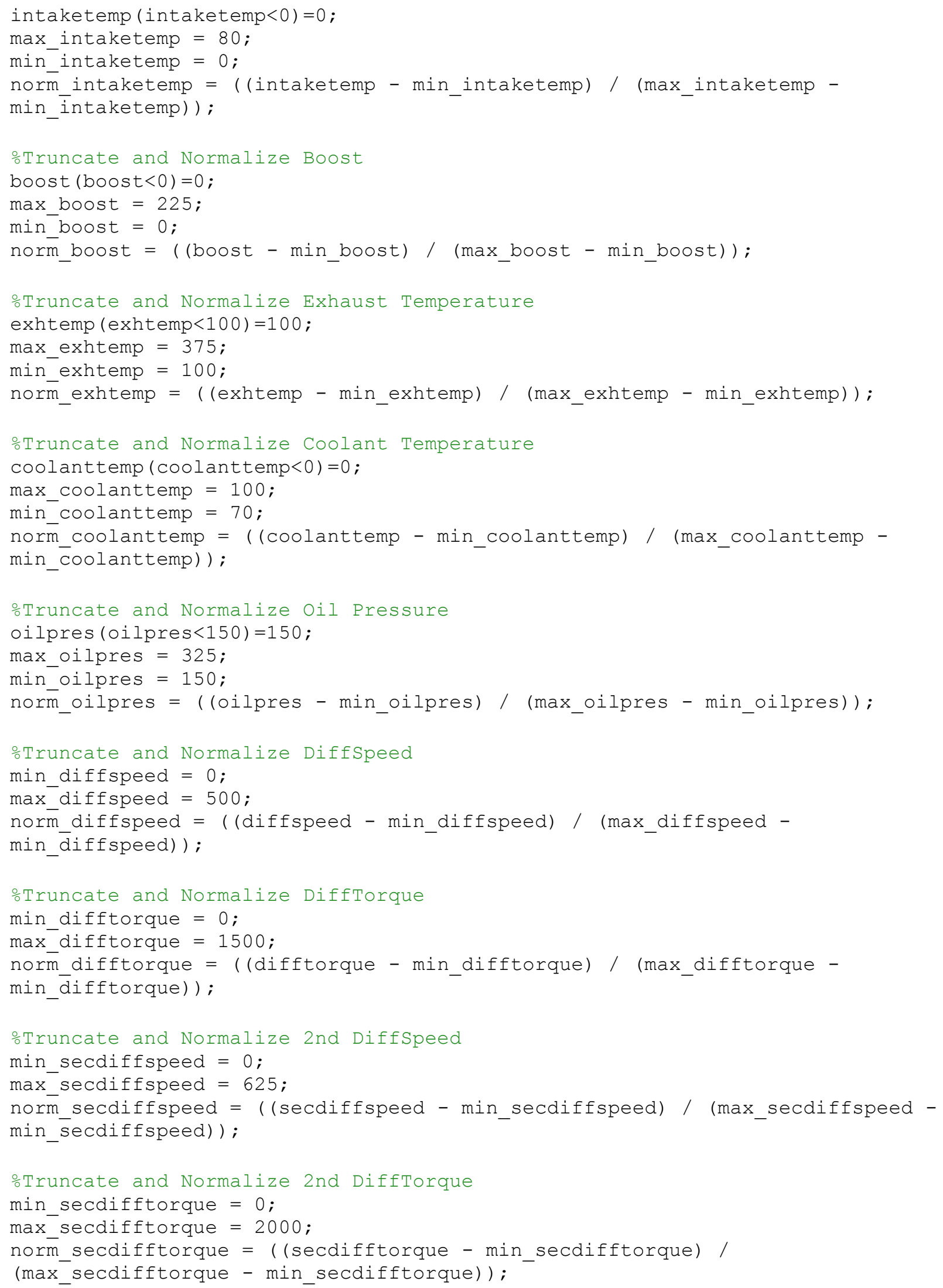




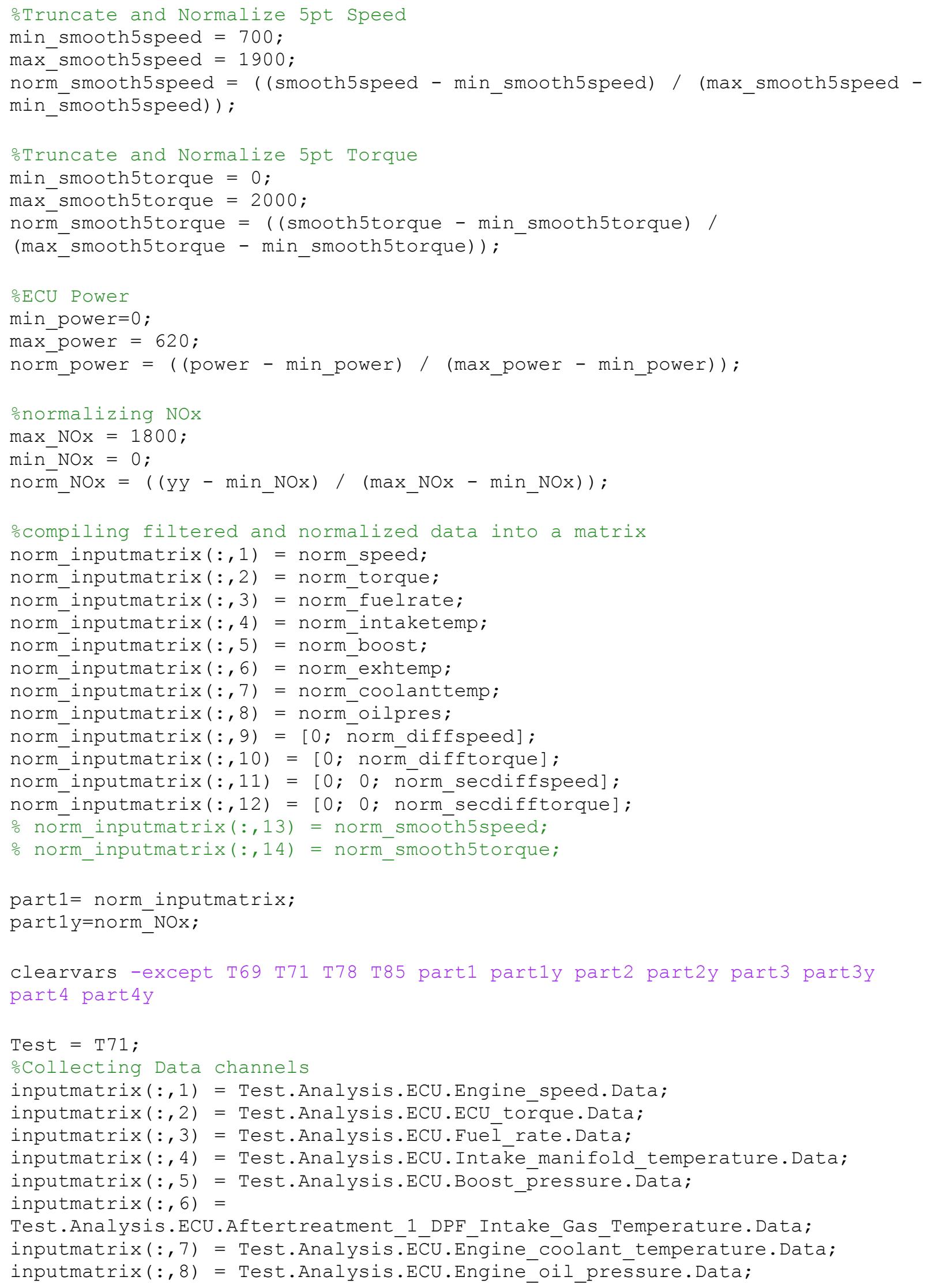




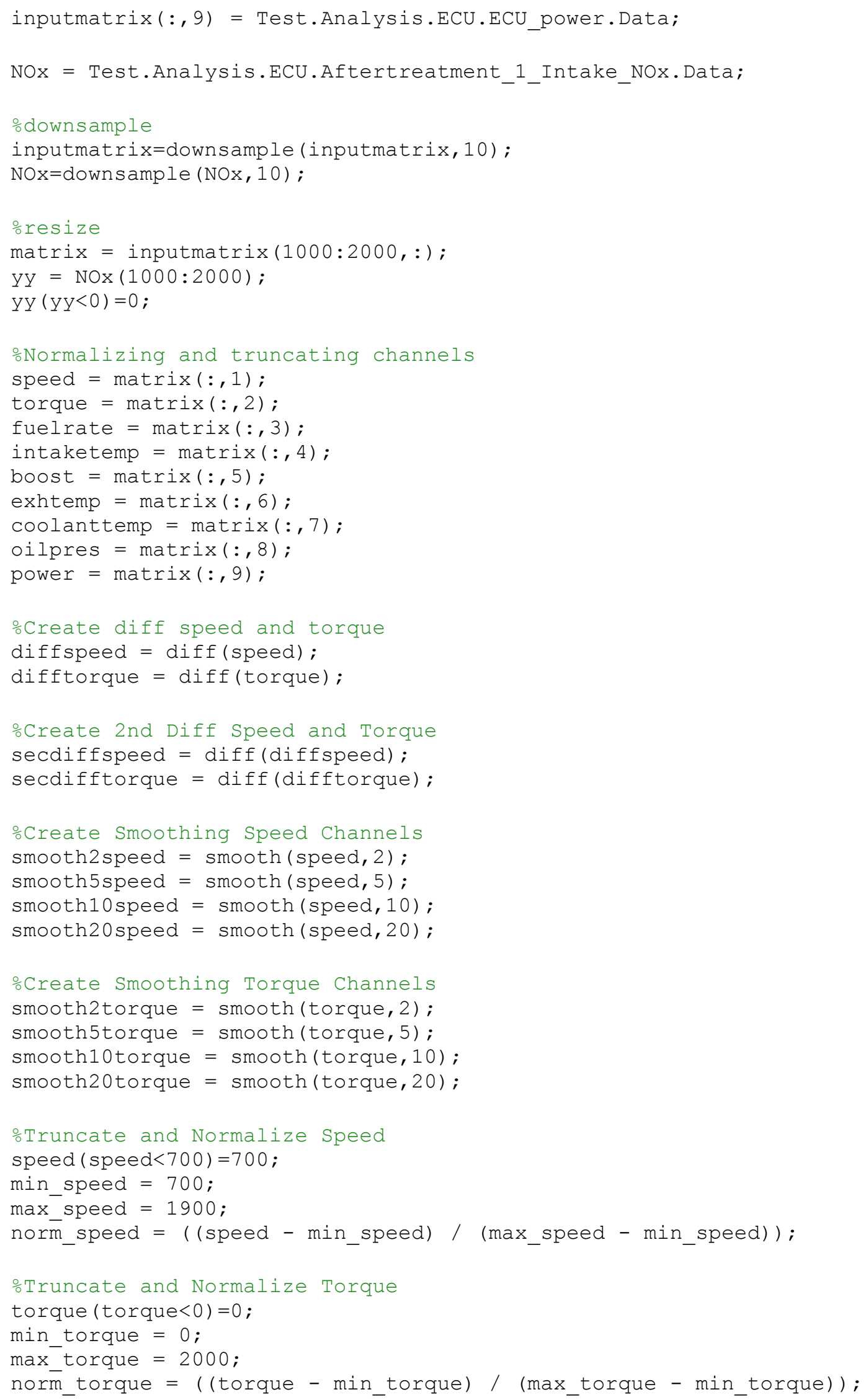




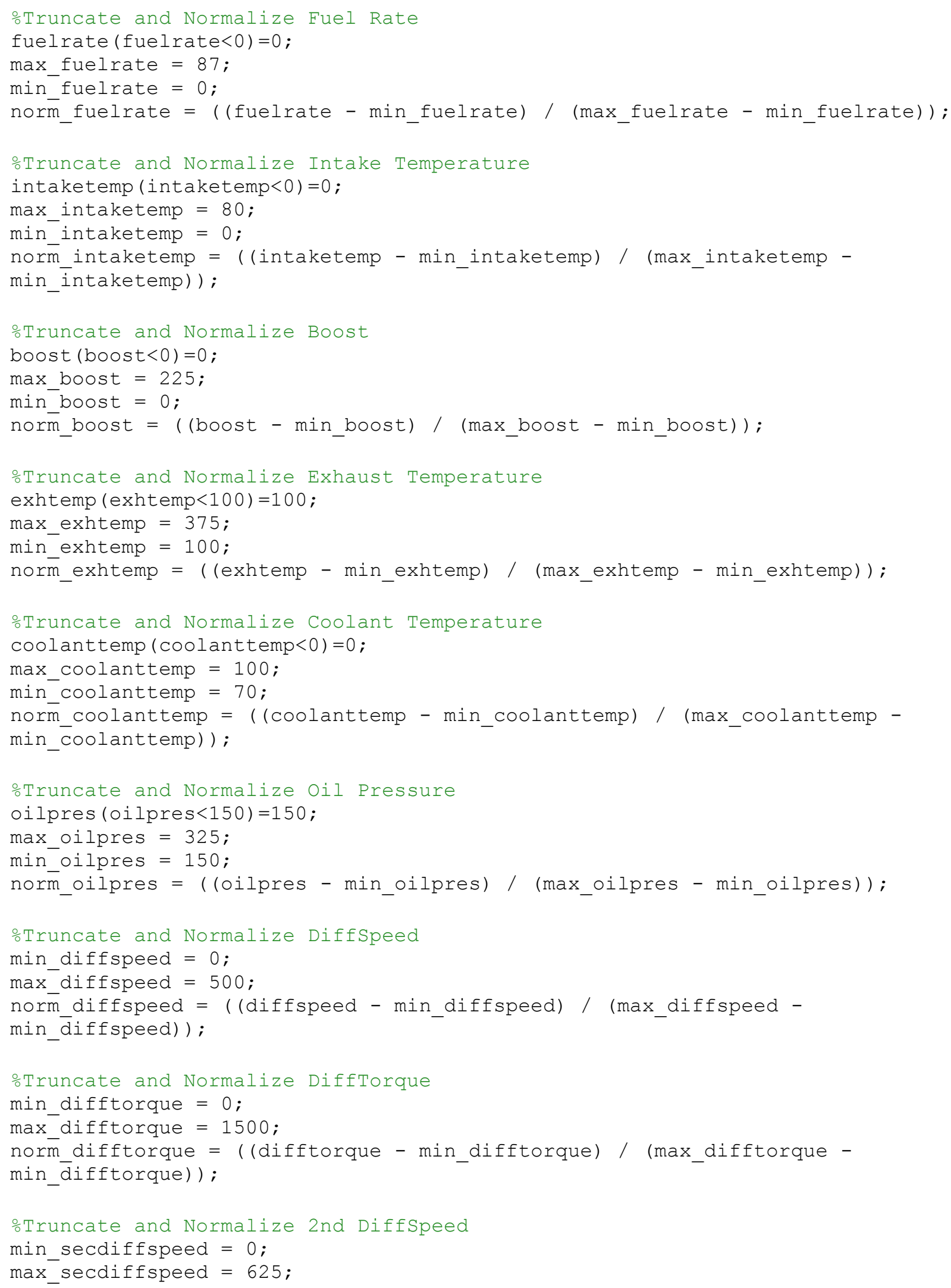




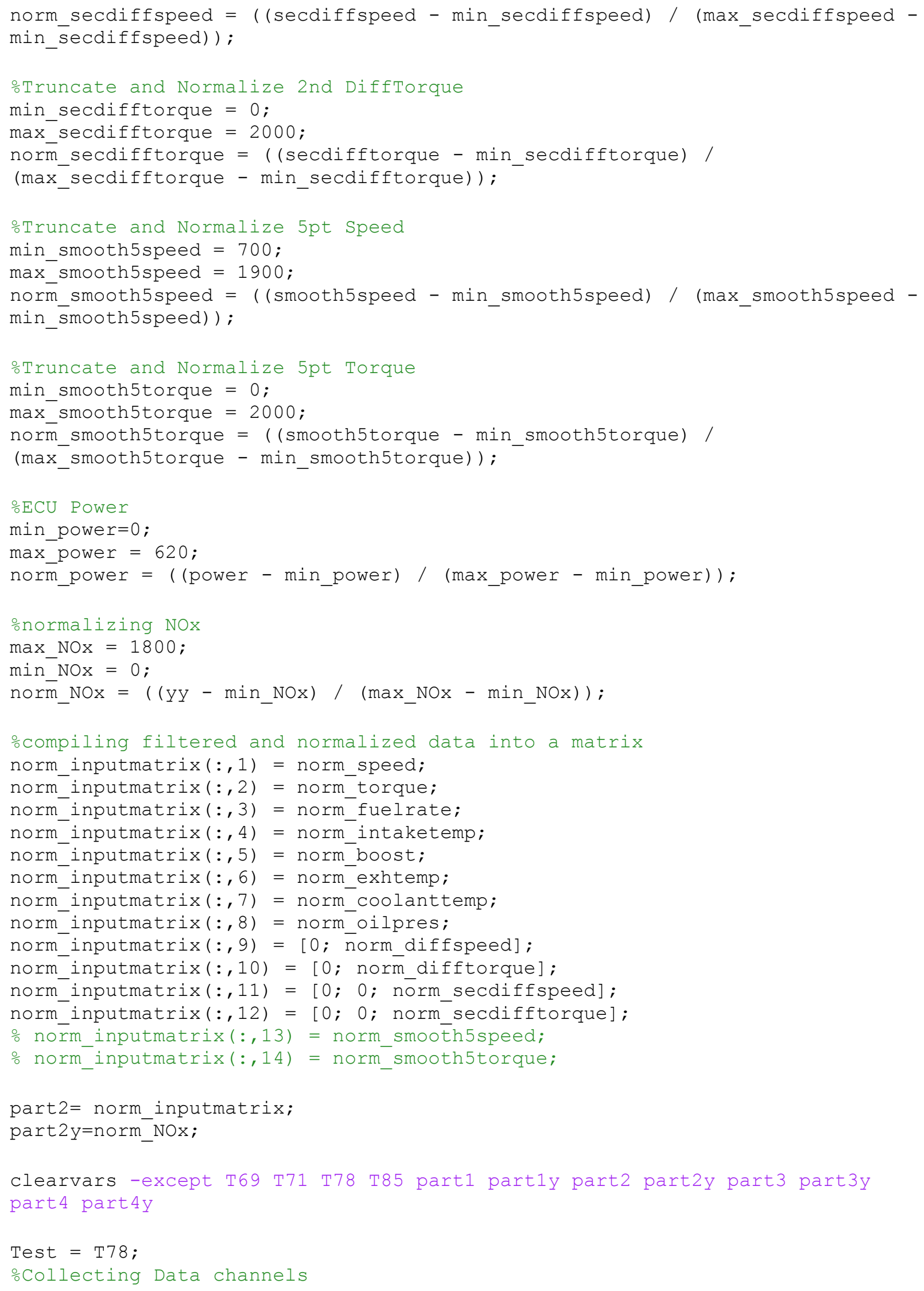




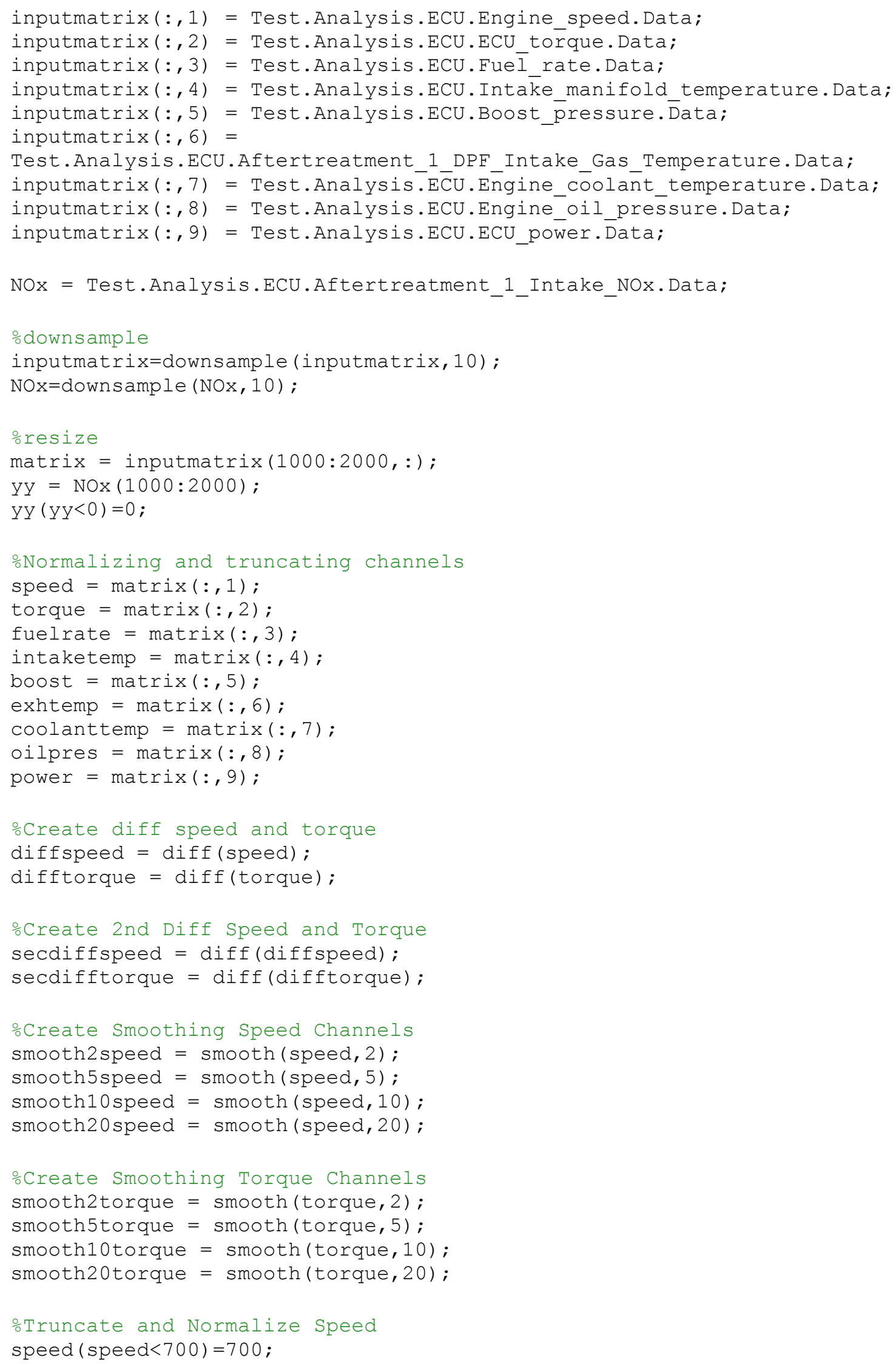




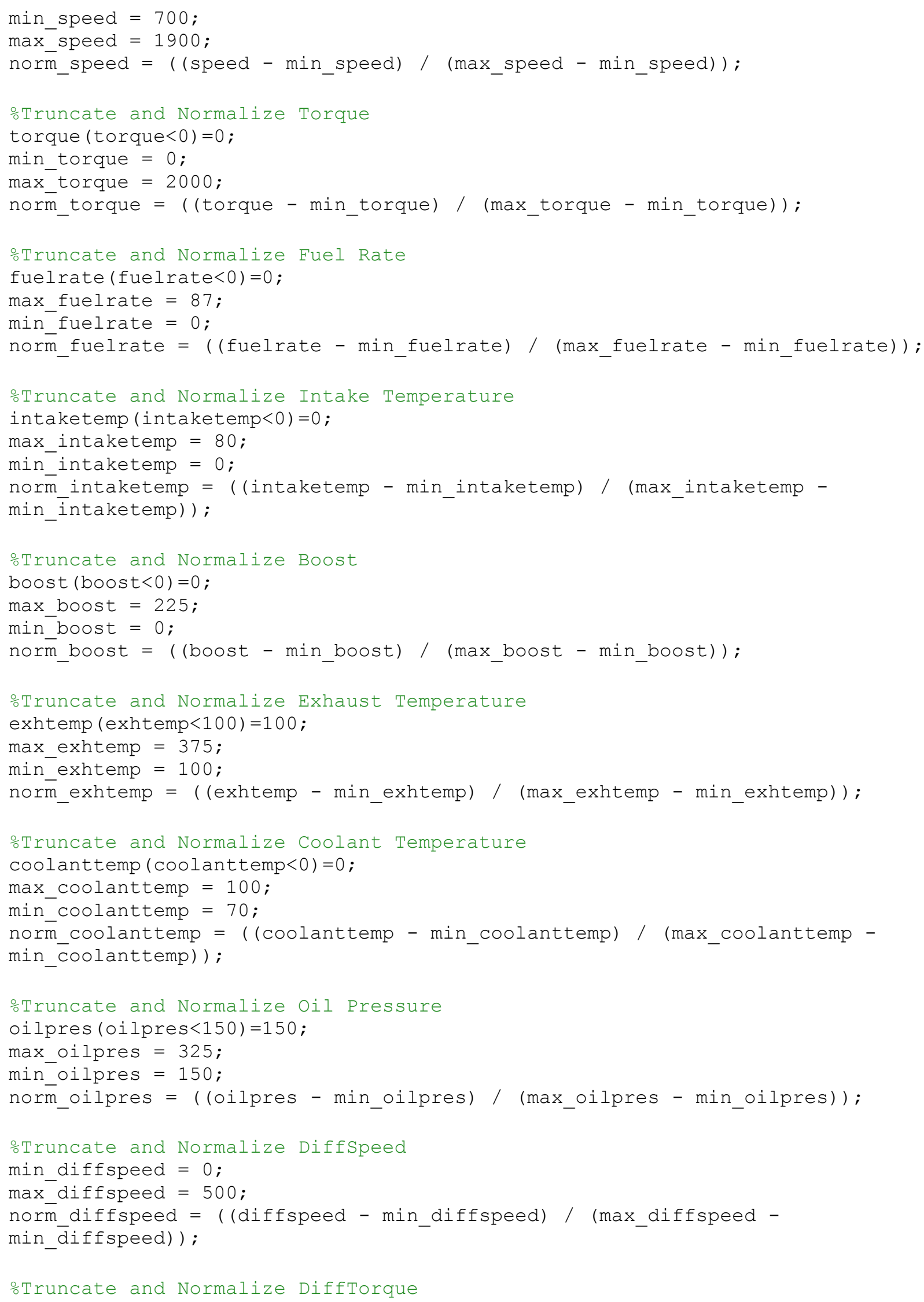




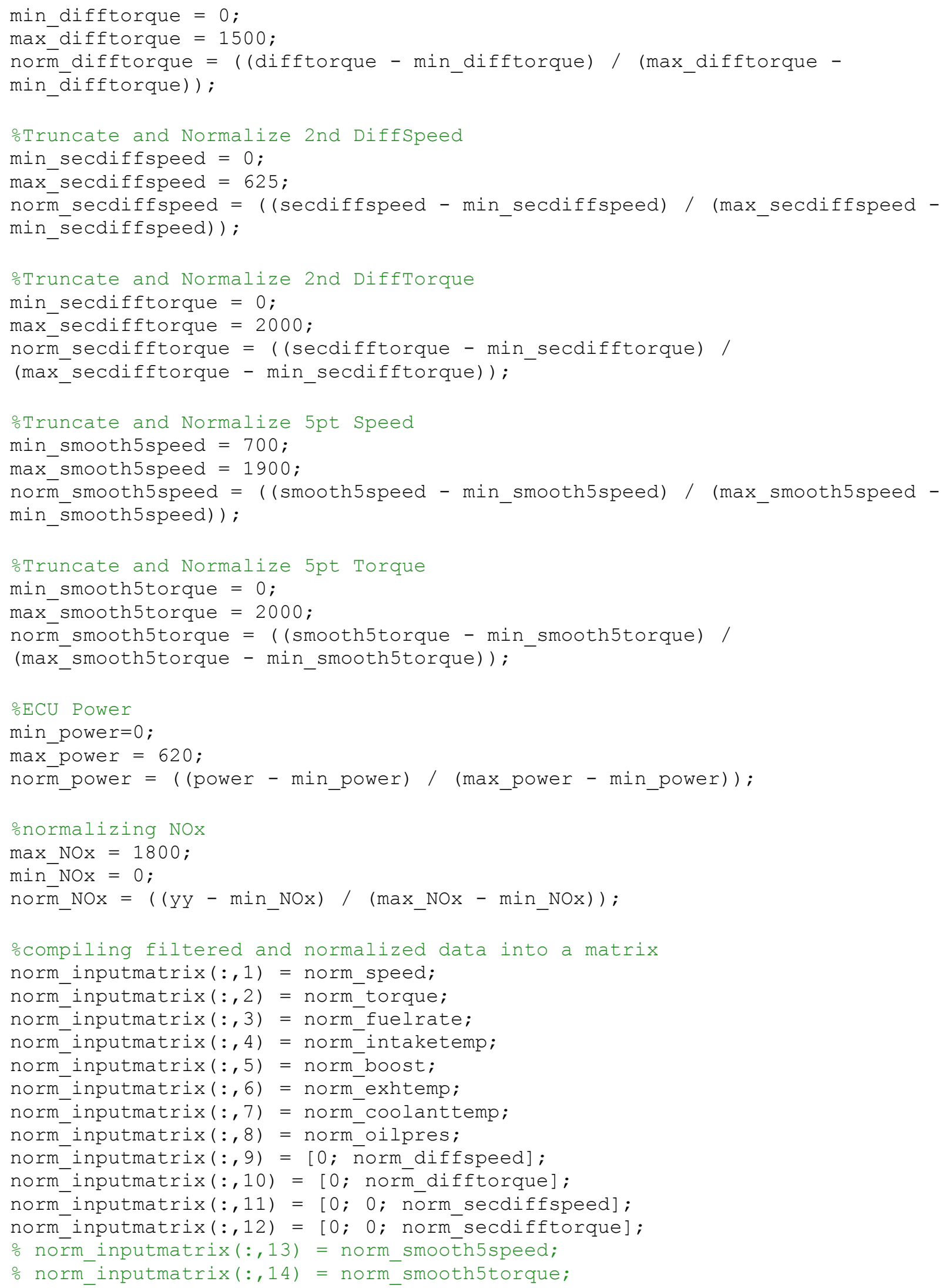




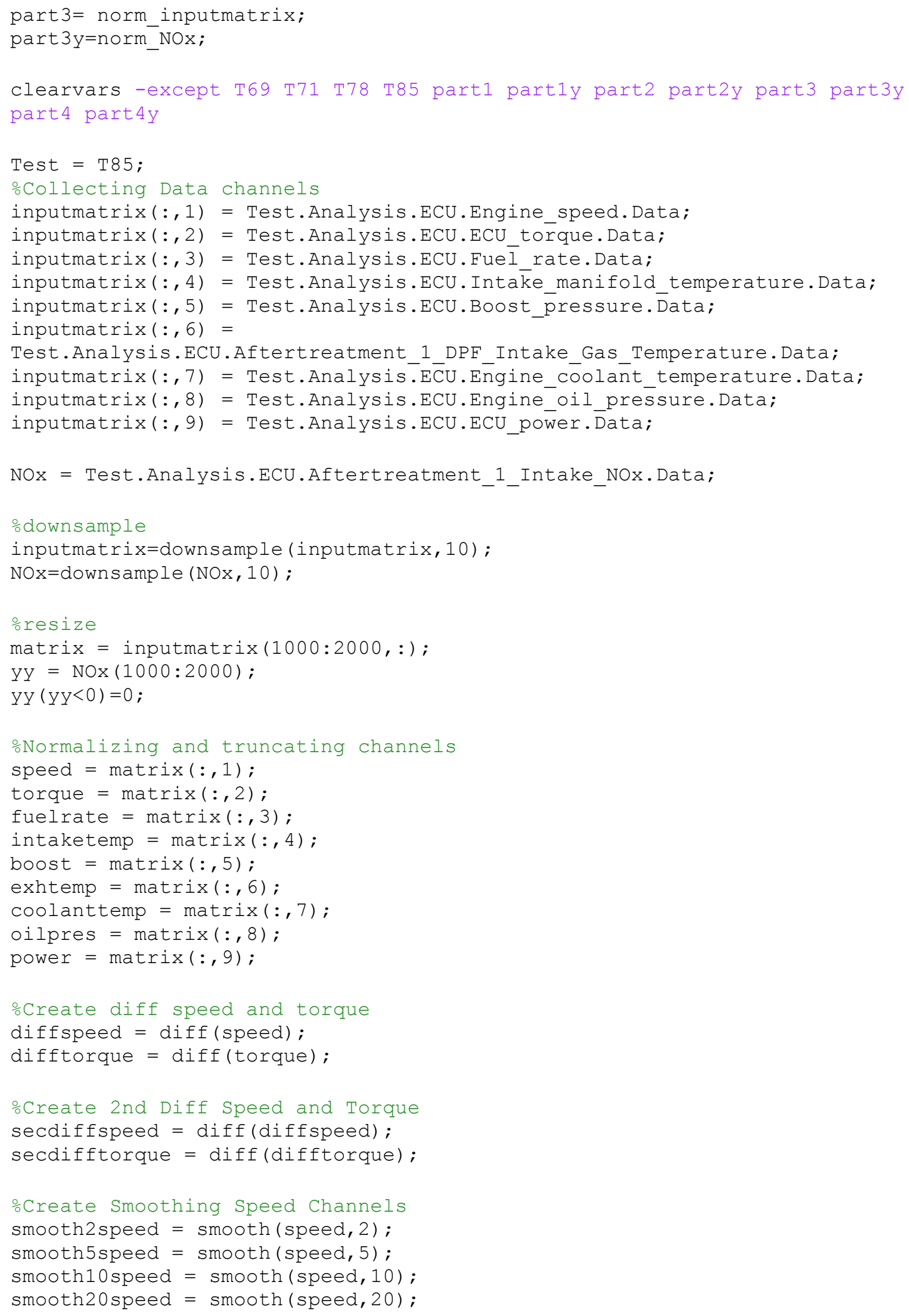




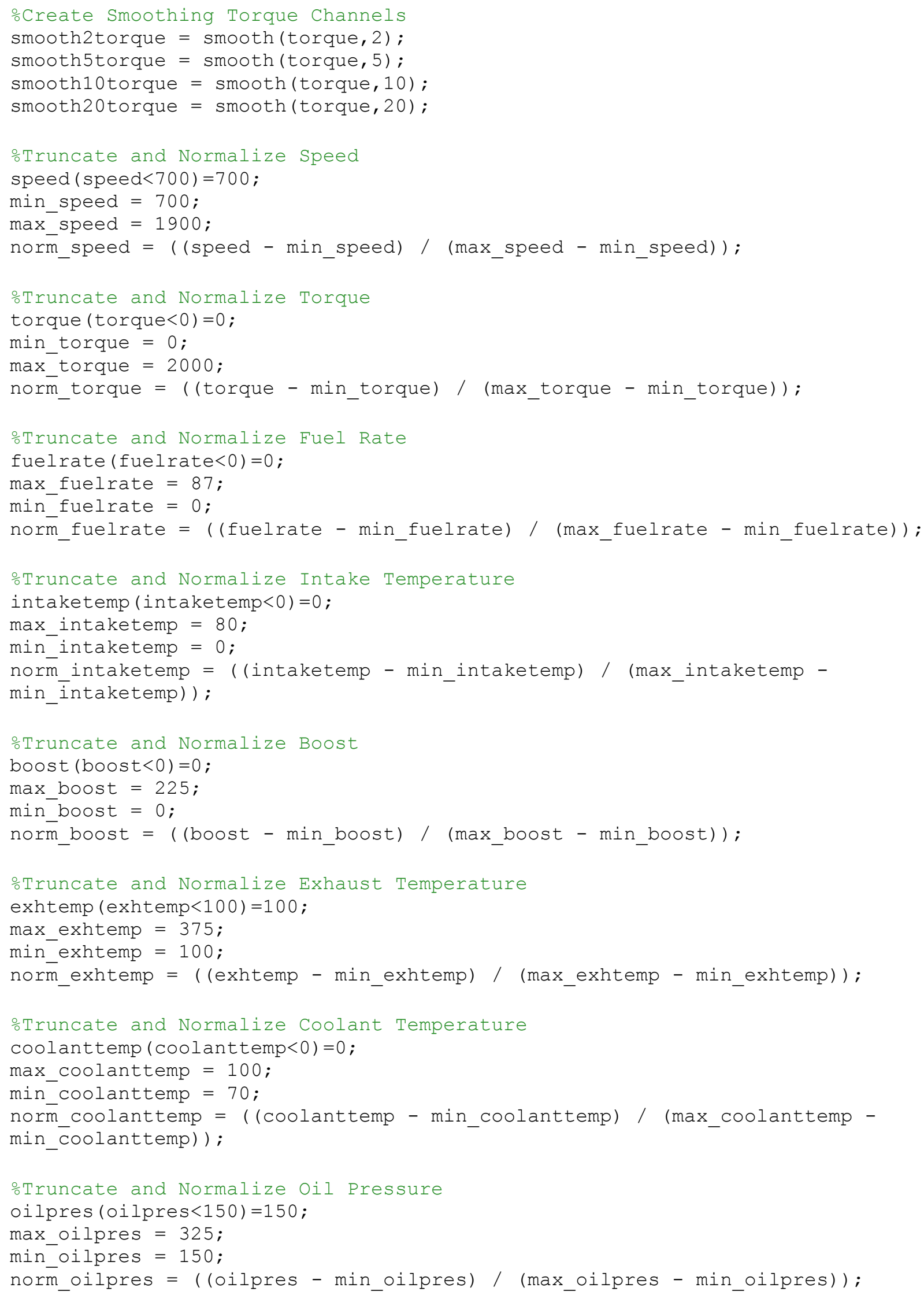




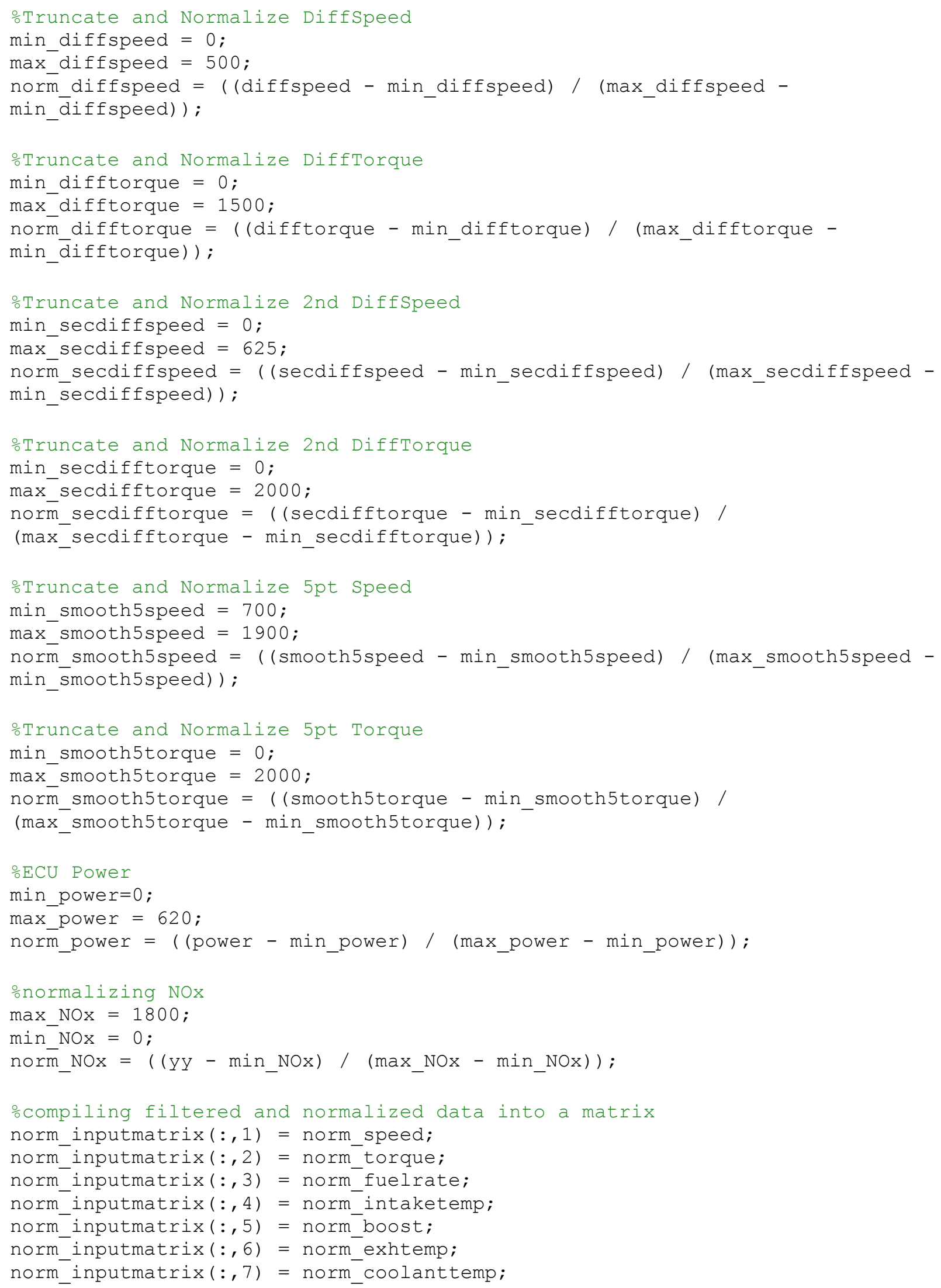




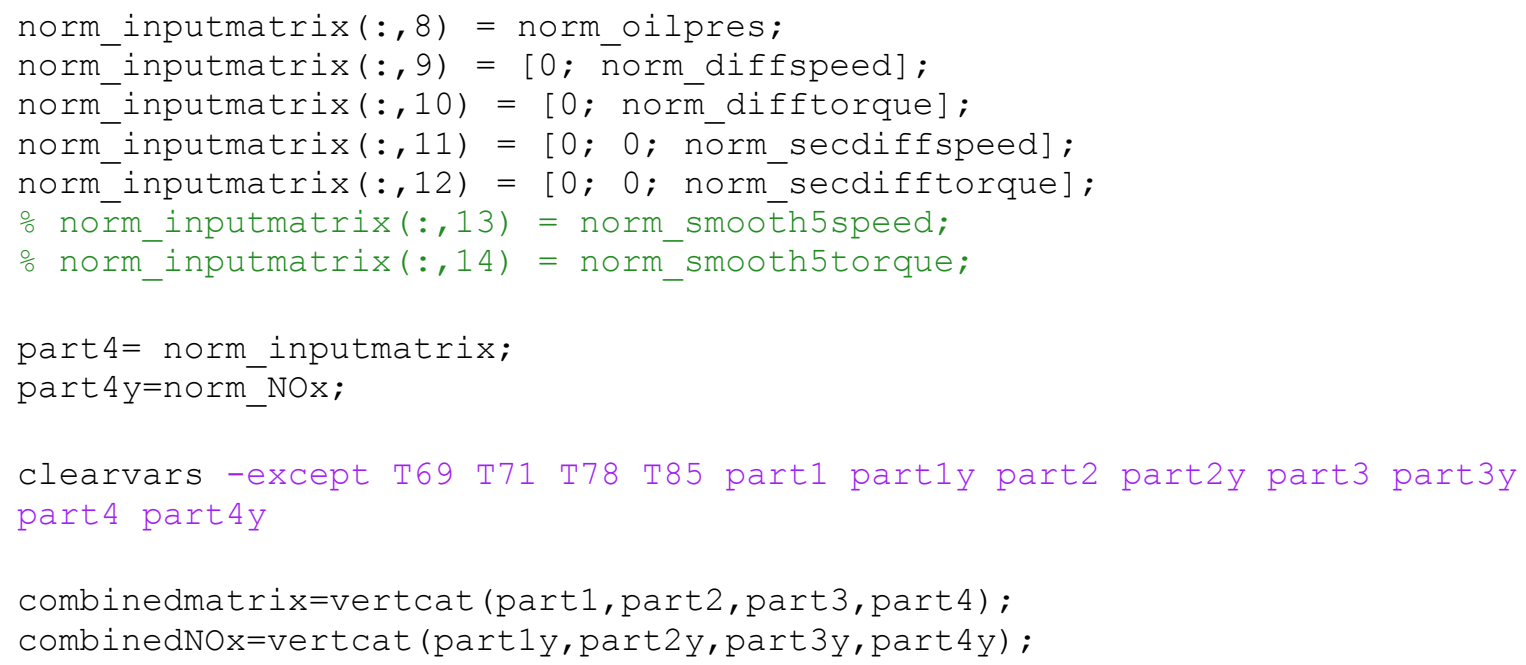

\subsection{APPENDIX C}

Appendix $C$ shows the Preprocessing code. This code is what was used to collect, normalize, and format the desired inputs for all other data sets that were used for validation testing.

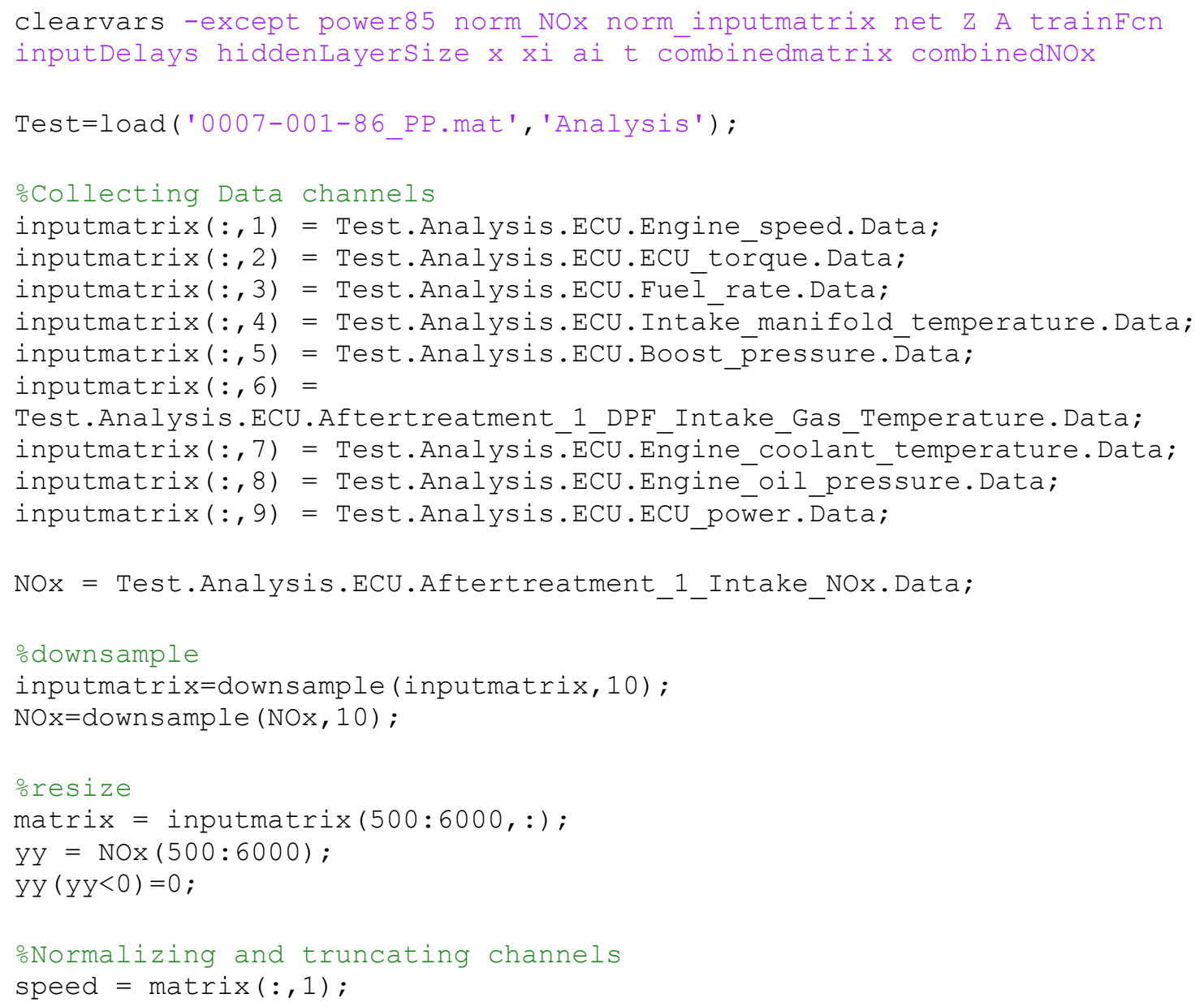




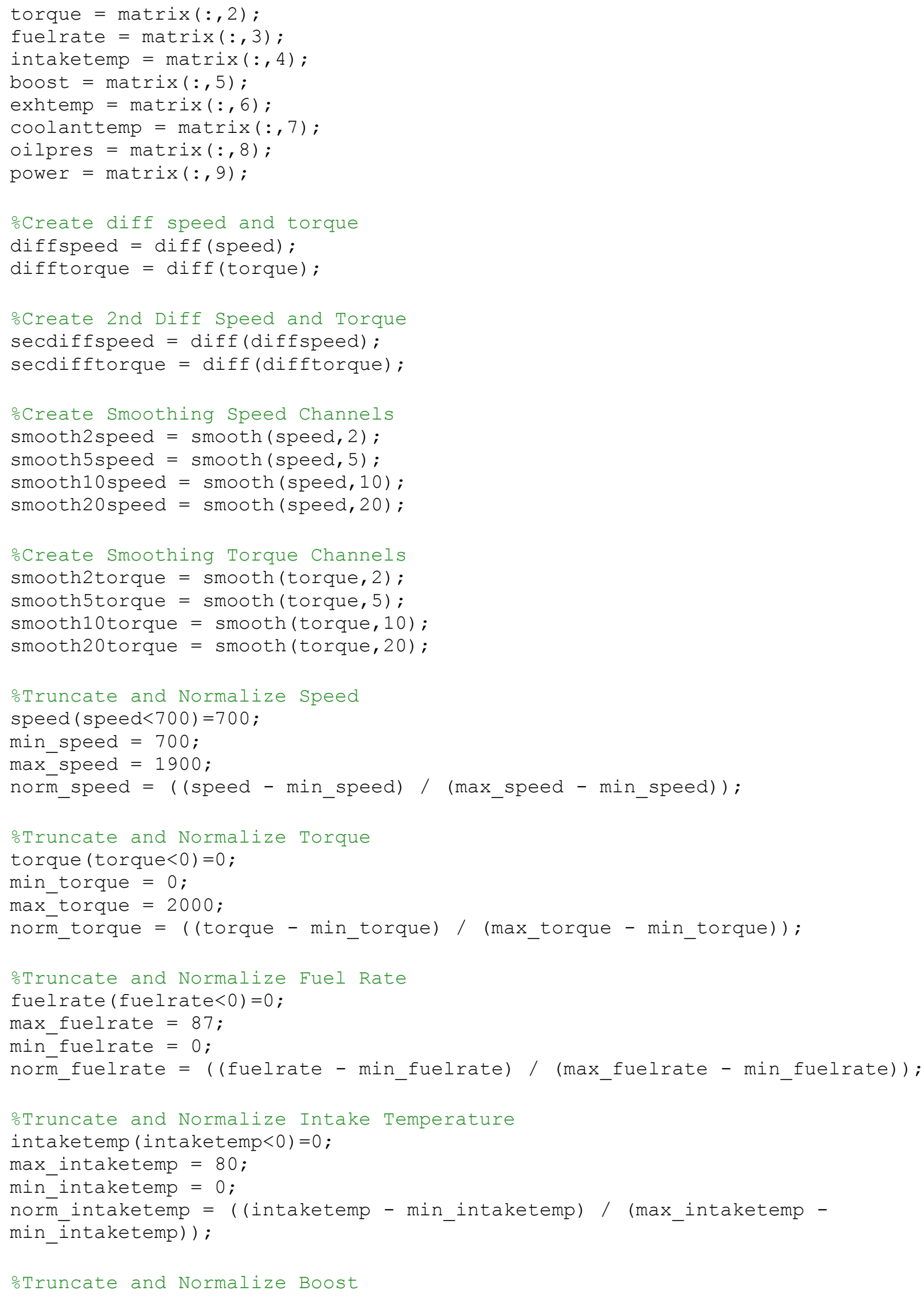




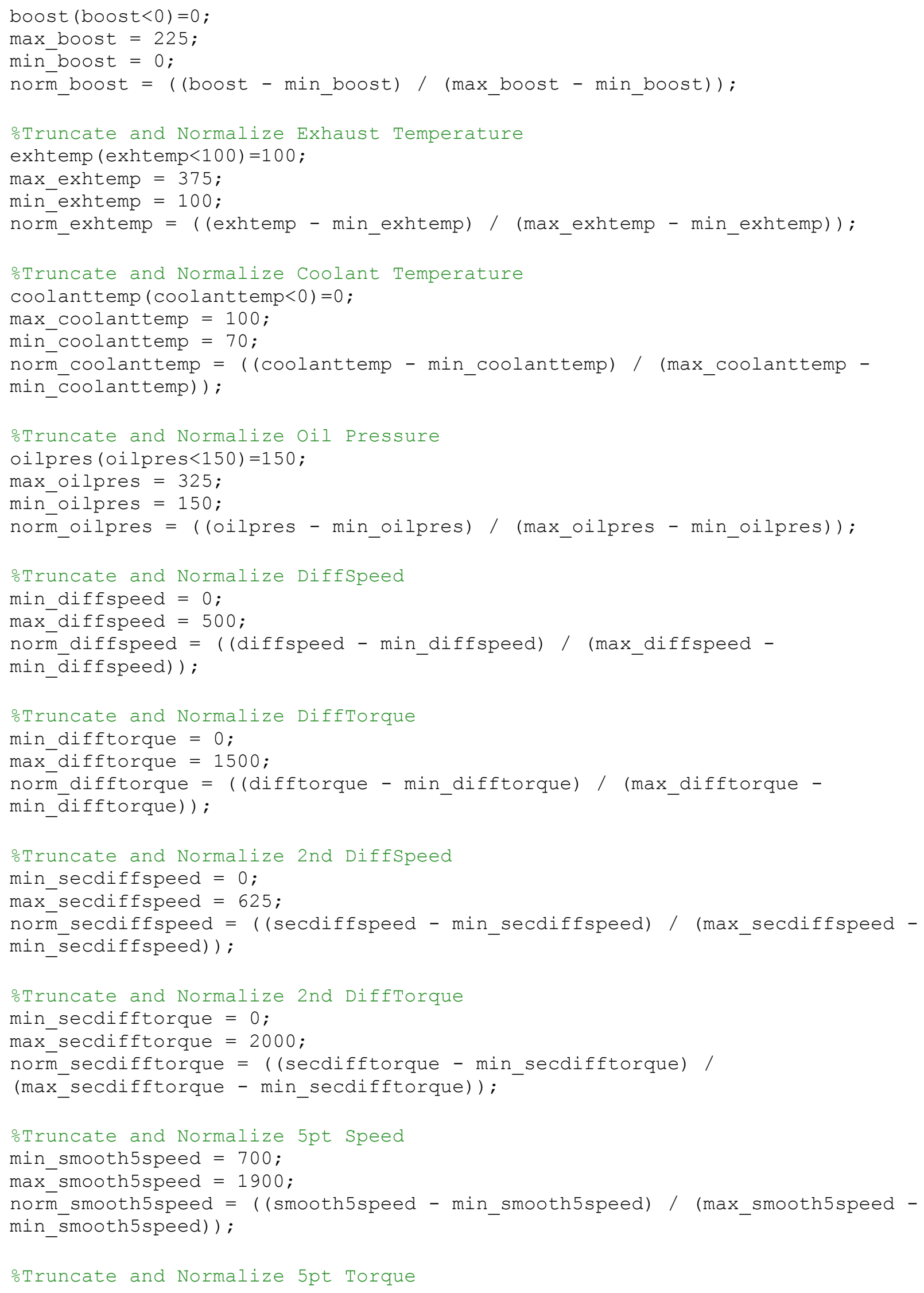




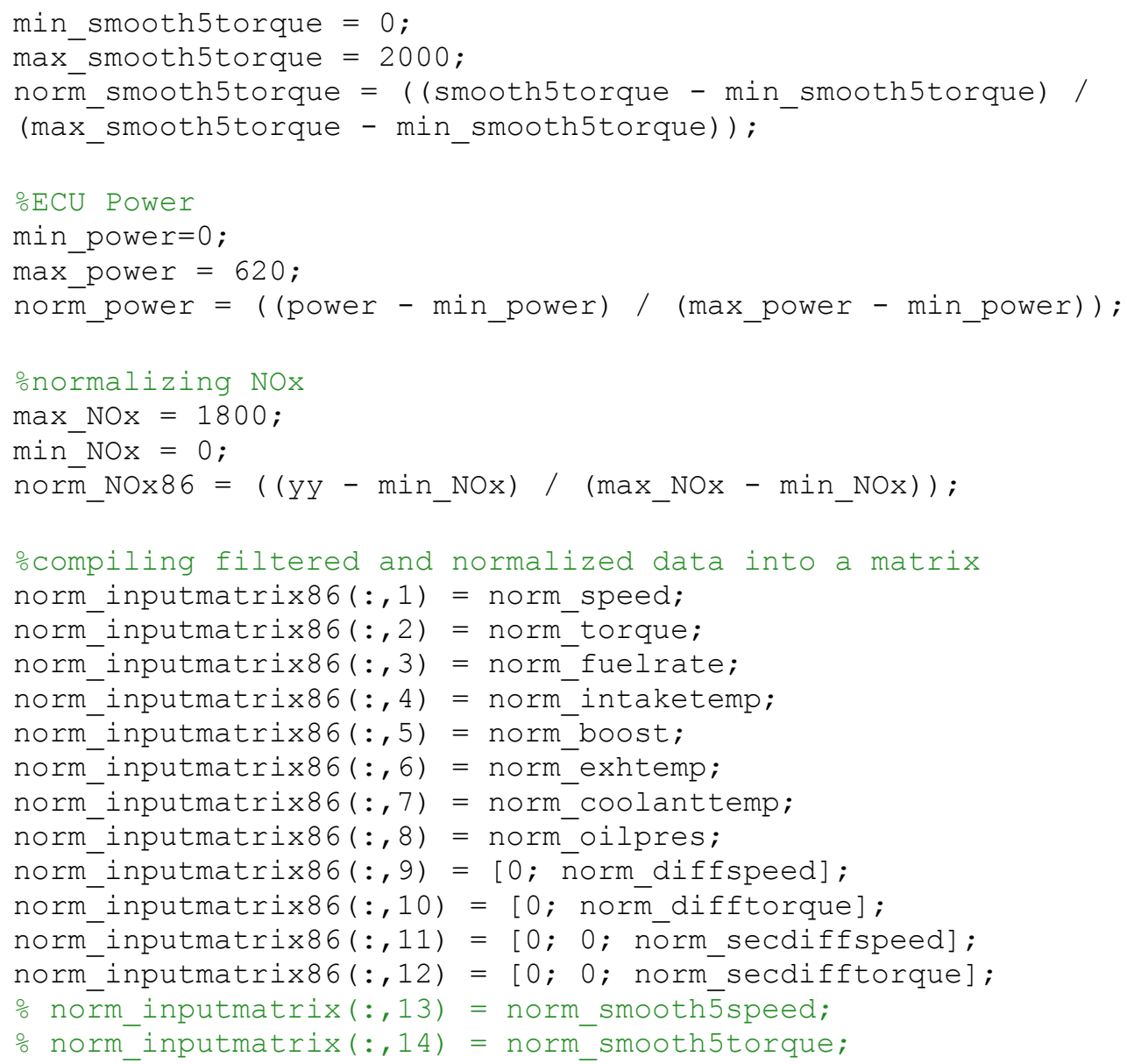

\subsection{APPENDIX D}

Appendix D includes the NOx Prediction code. This code imports the training data set and validation data set, and then sets the training function, input delay, and hidden layer size. This code then sets the initial weights for the neural network, trains the neural network, and then performs the prediction. The code then creates plots of the data and performs the correspondence calculation as well as the total error, and error in 200 second increments.

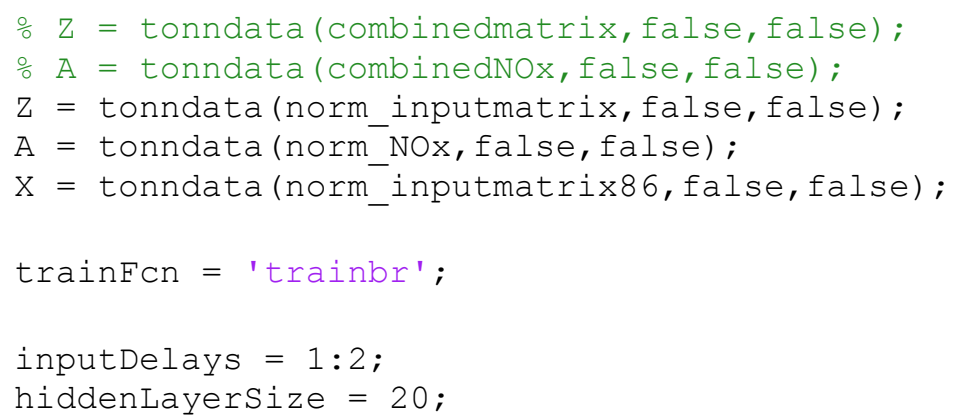




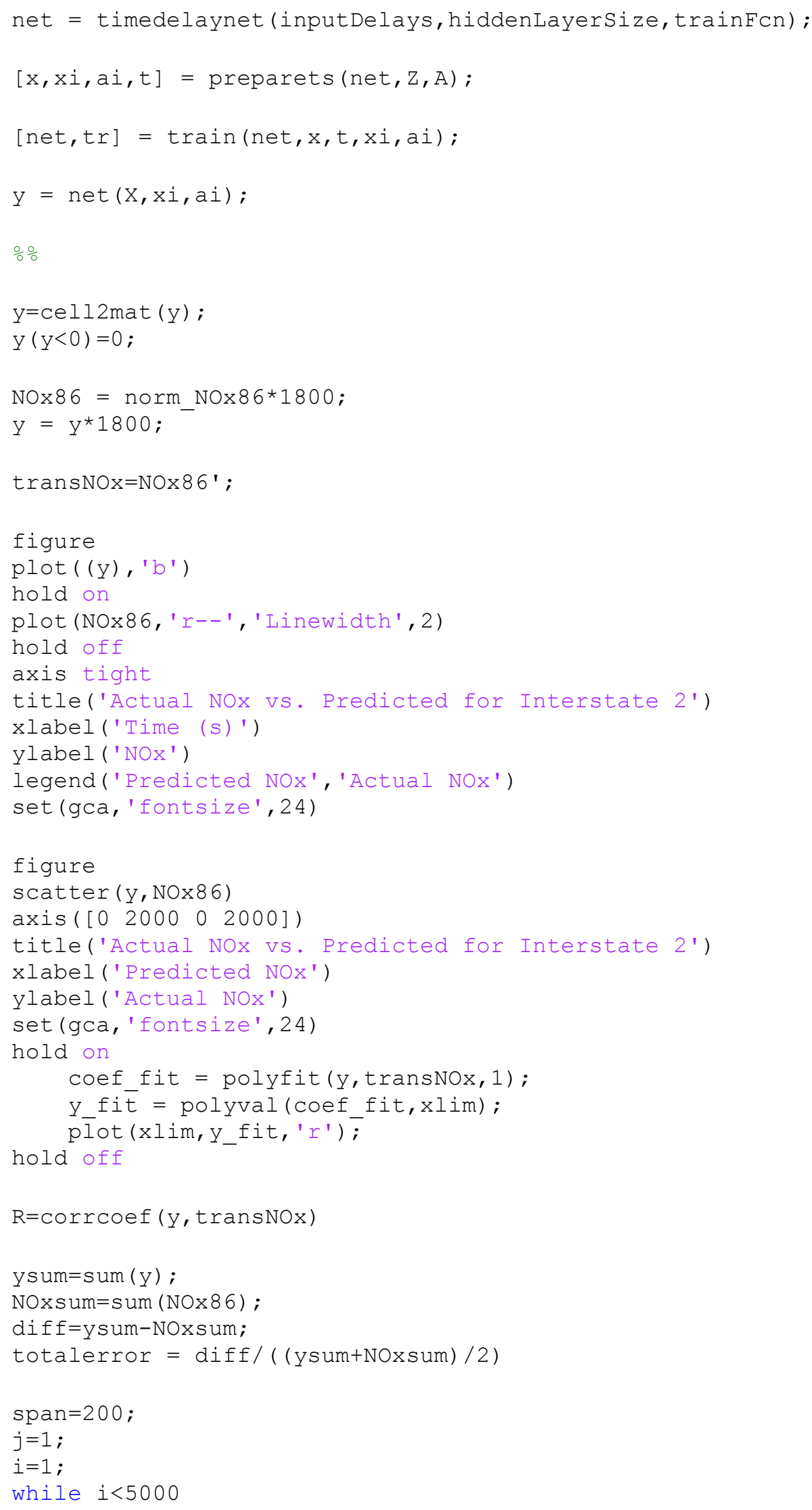




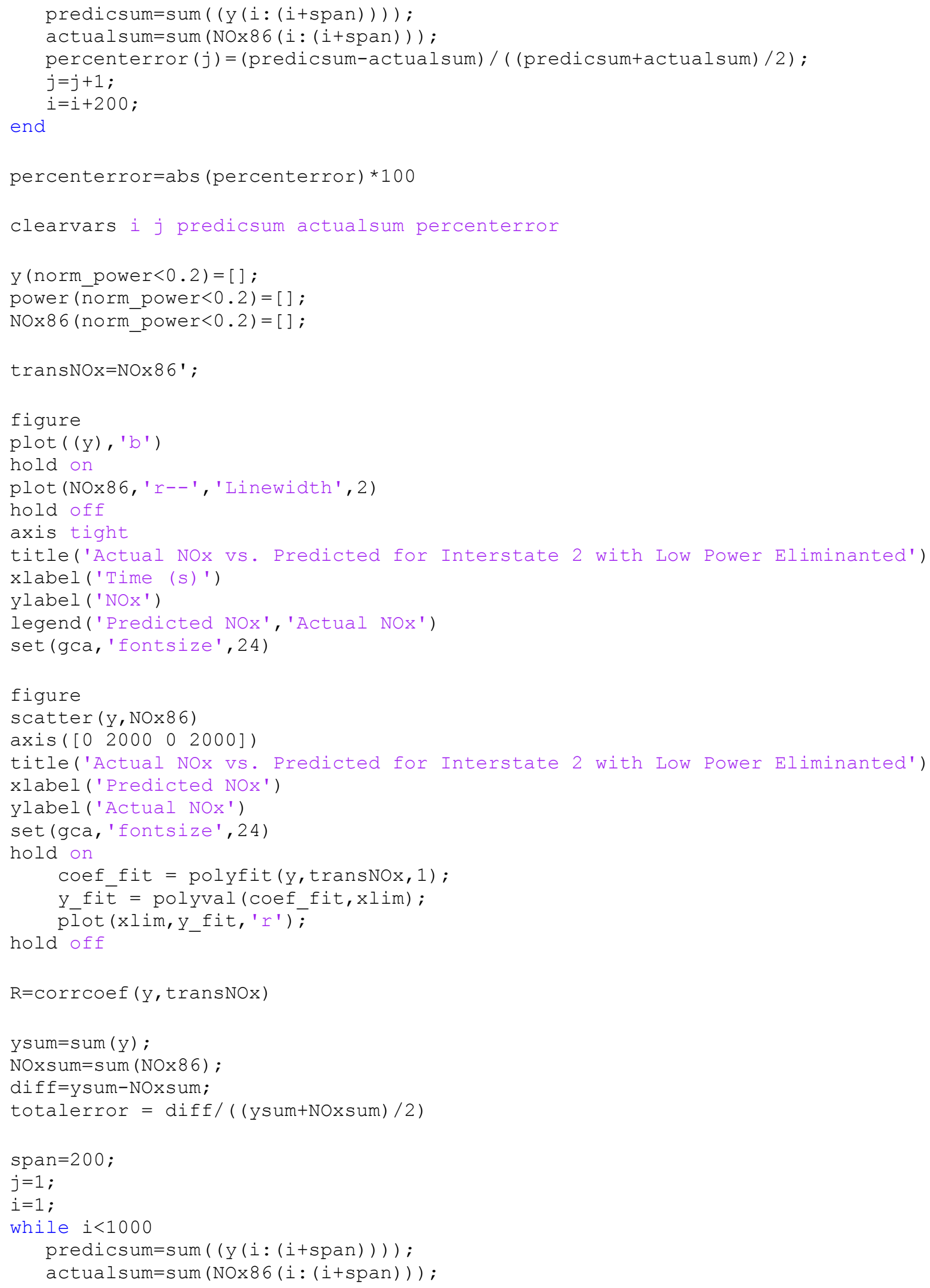




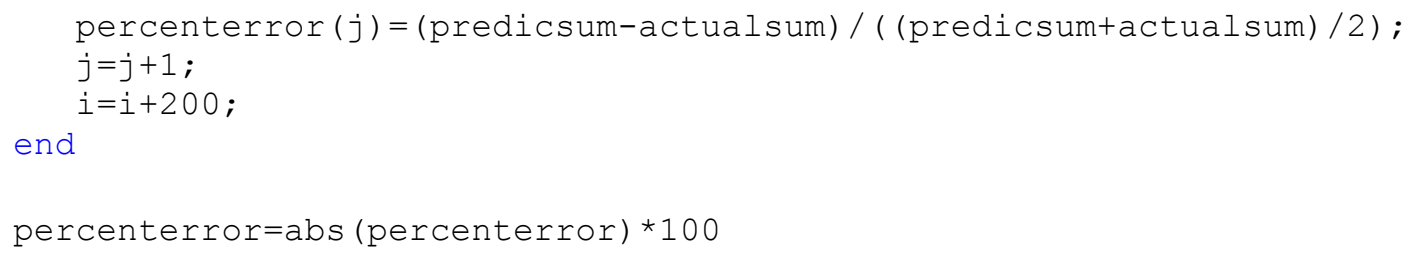

\subsection{APPENDIXE}

Appendix E shows the NOx Fault Simulation code. This code is what was used to insert a simulated NOx sensor fault into the data. This code creates a step, ramp, and square function failure.

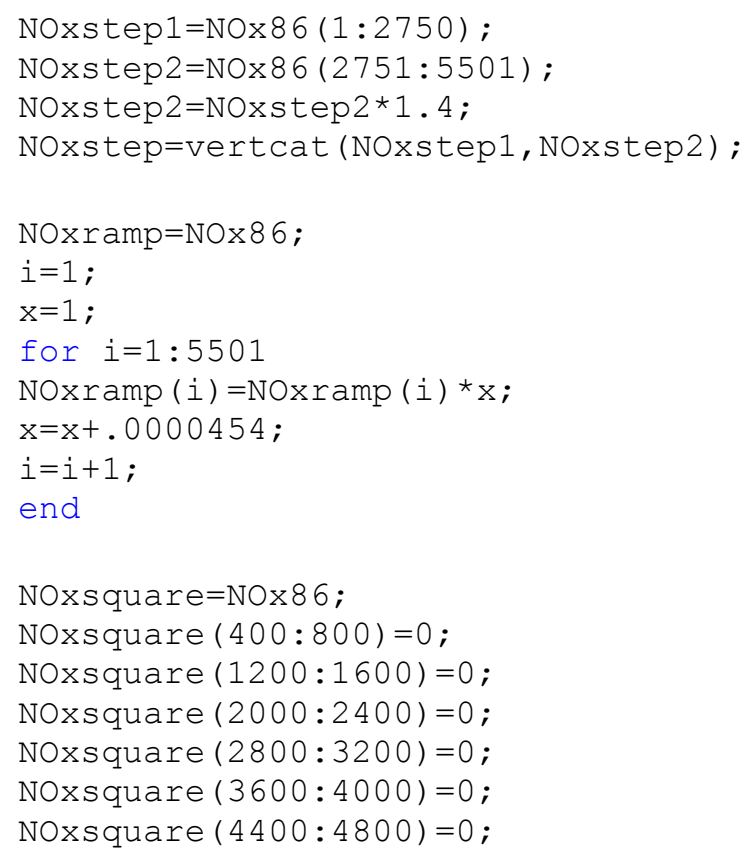

\subsection{APPENDIX F}

Appendix F shows the Boost Sensor Fault Simulation code. This code is what was used to insert a simulated boost pressure sensor fault into the data. This code creates a step, ramp, and square function failure.

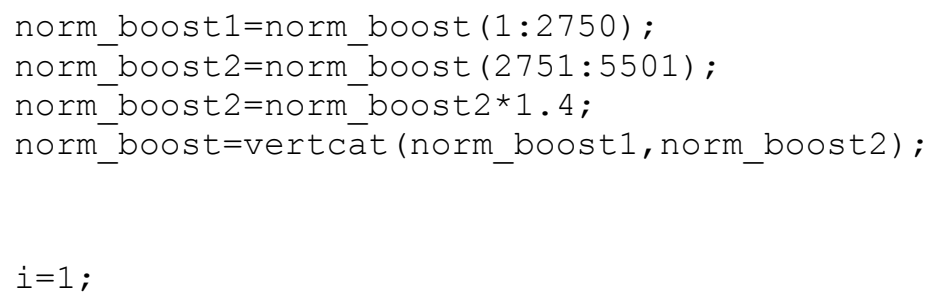


$\mathrm{x}=1$;

for $i=1: 5501$

norm_boost $(i)=$ norm_boost $(i){ }^{*} x$;

$\mathrm{x}=\mathrm{x}+.0000454$;

$i=i+1$;

end

norm_boost $(400: 800)=0$;

norm_boost $(1200: 1600)=0$;

norm_boost $(2000: 2400)=0$;

norm_boost $(2800: 3200)=0$;

norm_boost $(3600: 4000)=0$;

norm_boost $(4400: 4800)=0$;

\subsection{APPENDIX G}

Appendix $G$ includes the time dependent NOx values for the neural network prediction compared to the actual values read by the NOx sensor when the neural network was trained with the Highway 1 data set.

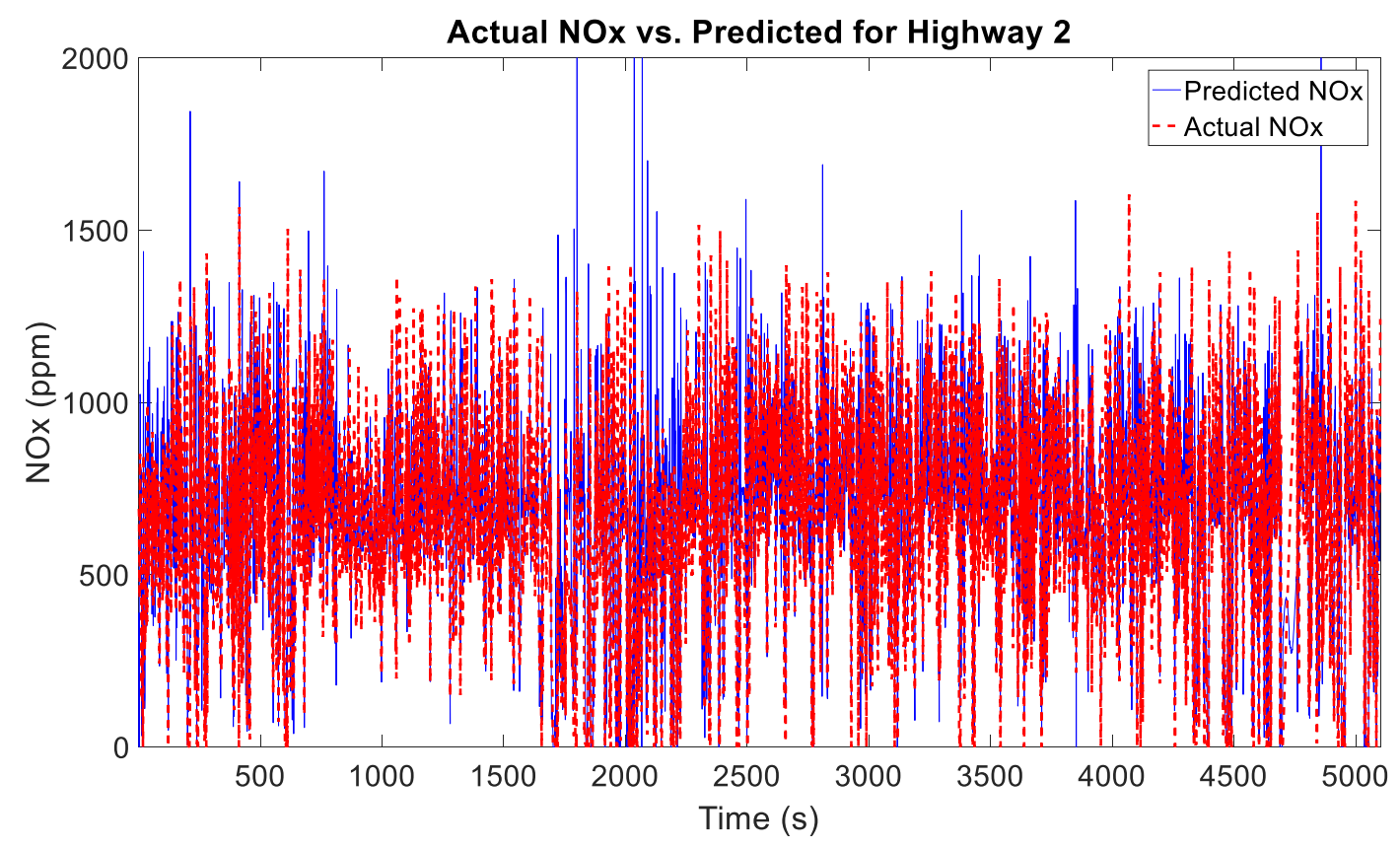



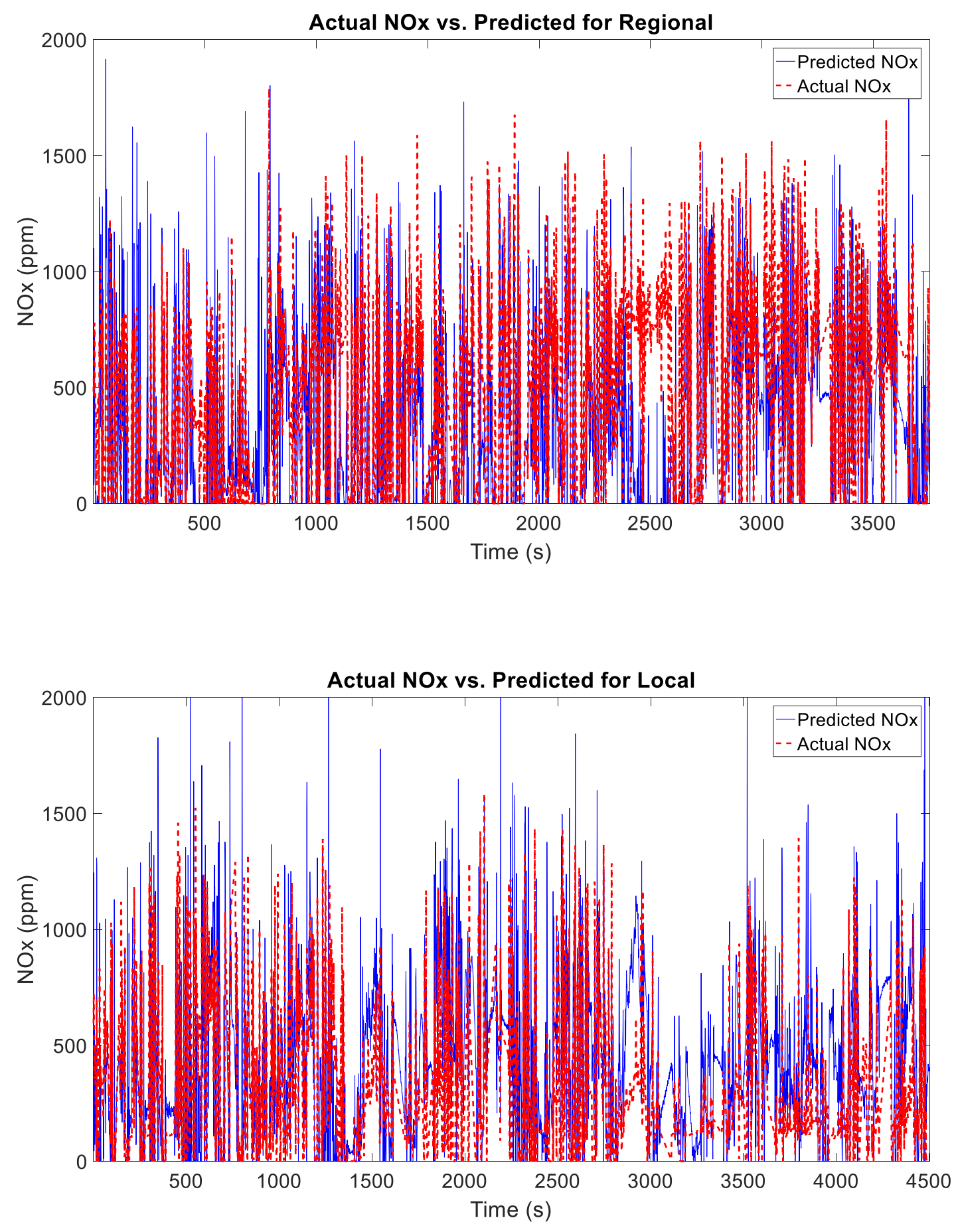

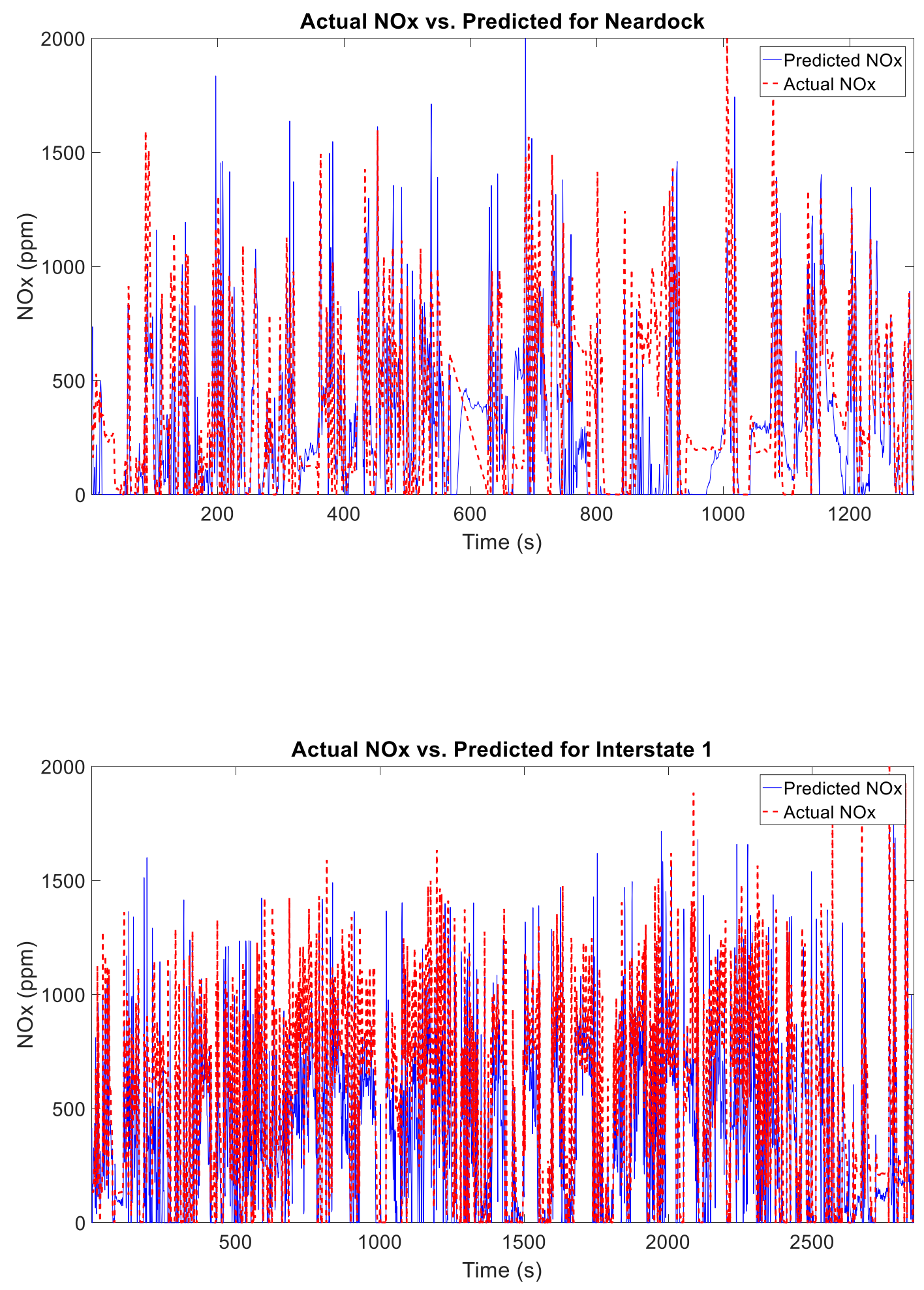


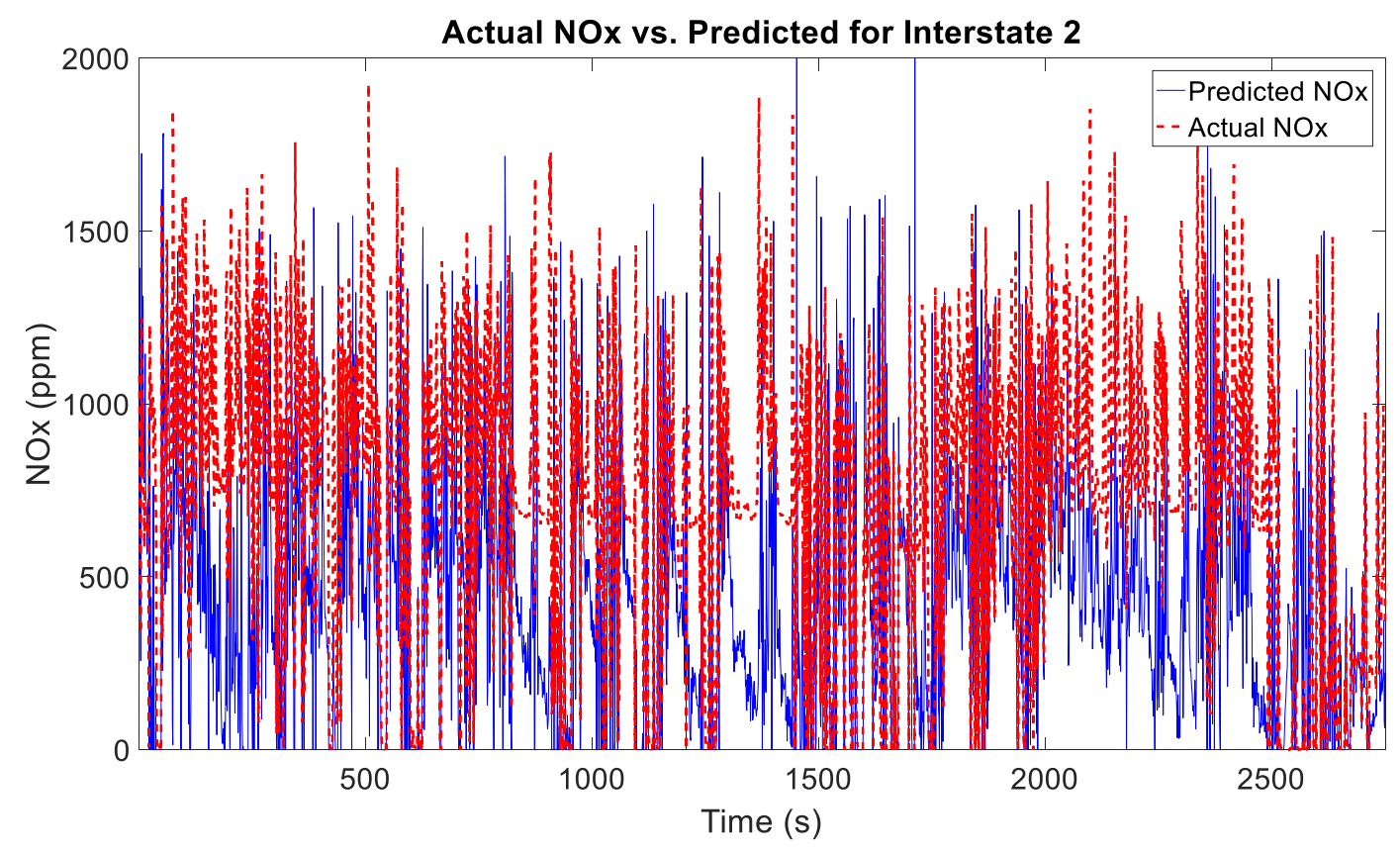

\subsection{APPENDIX H}

Appendix $\mathrm{H}$ includes the time dependent NOx values of the predicted versus actual for the neural network when it was trained with training data set 2 .

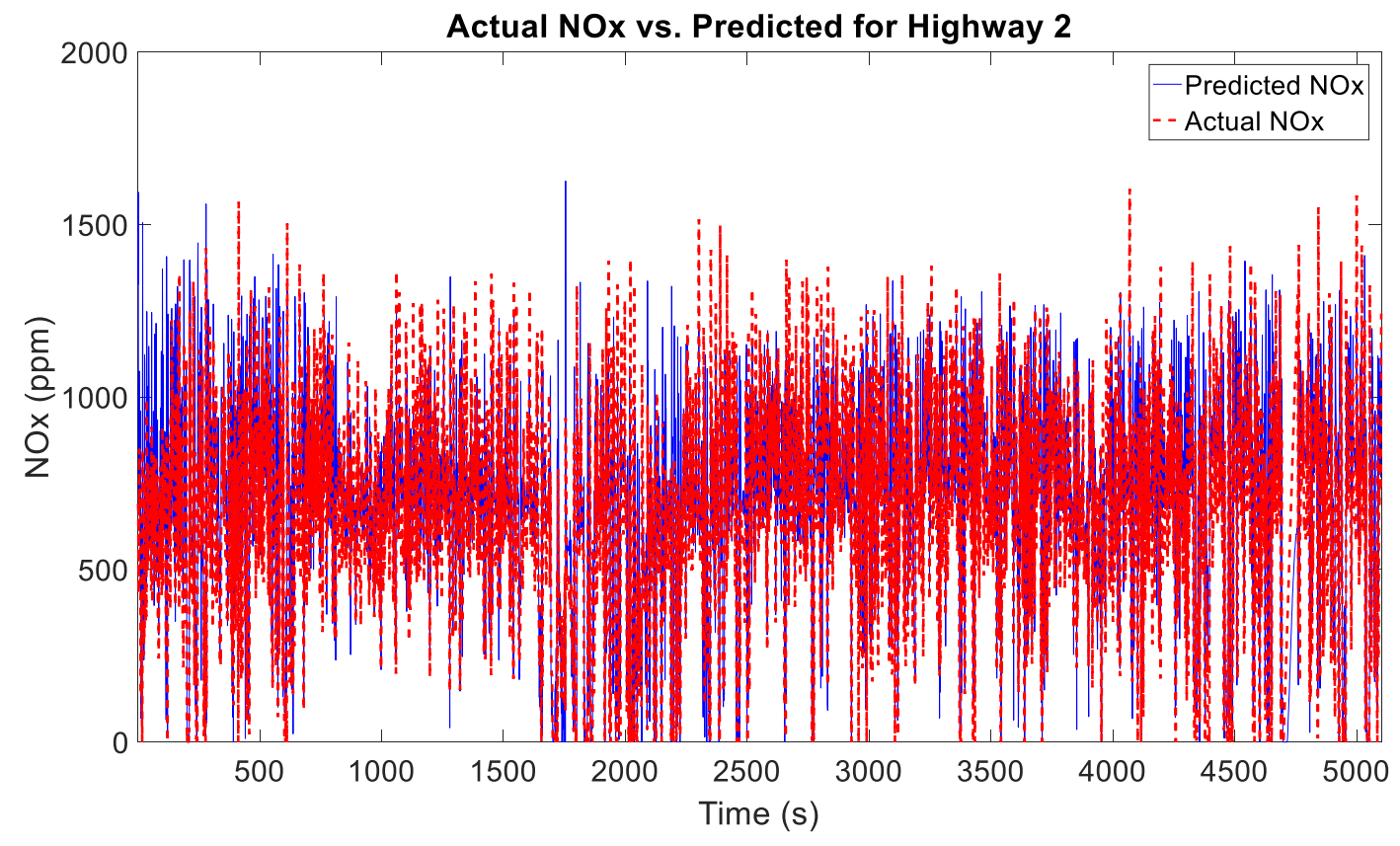



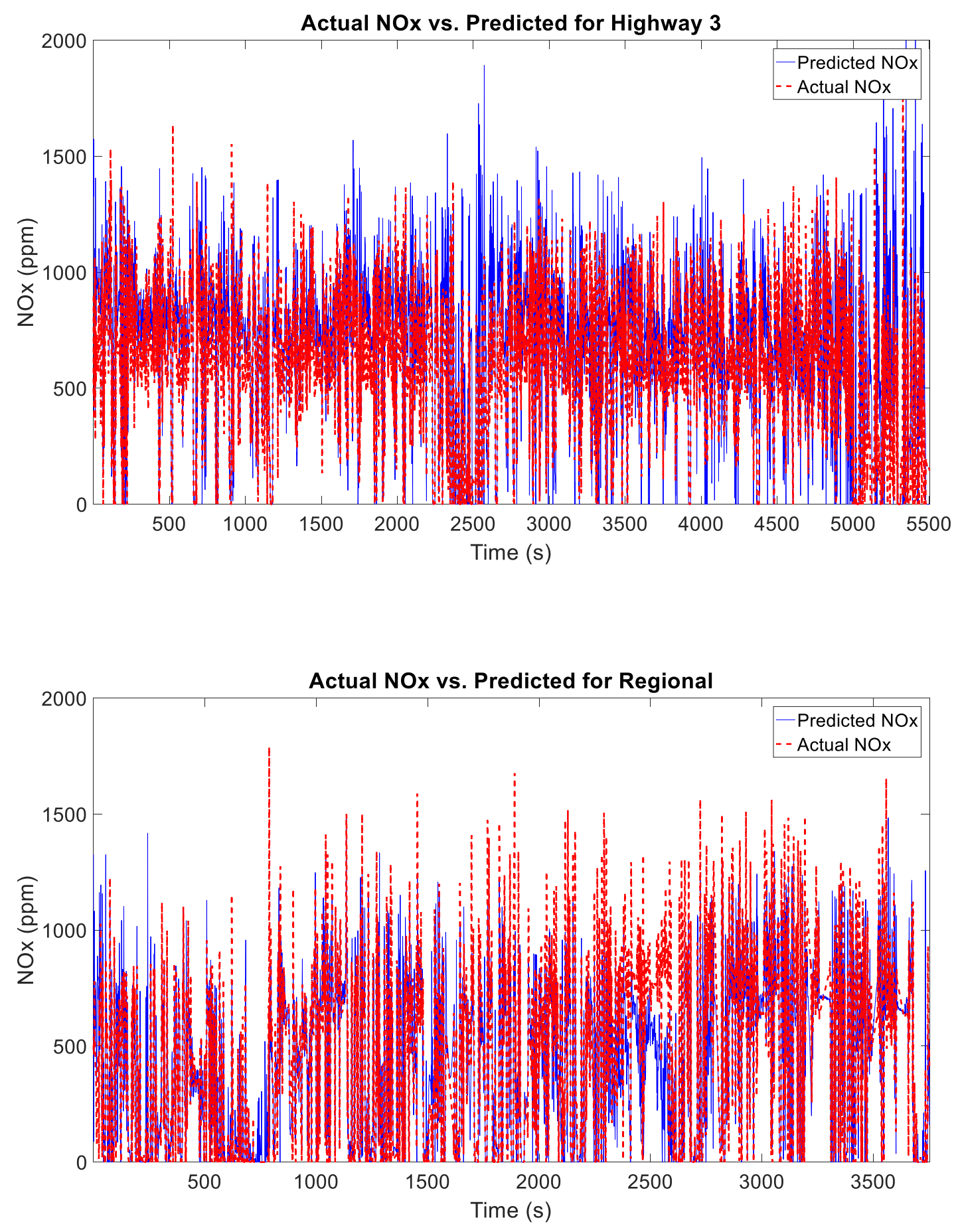

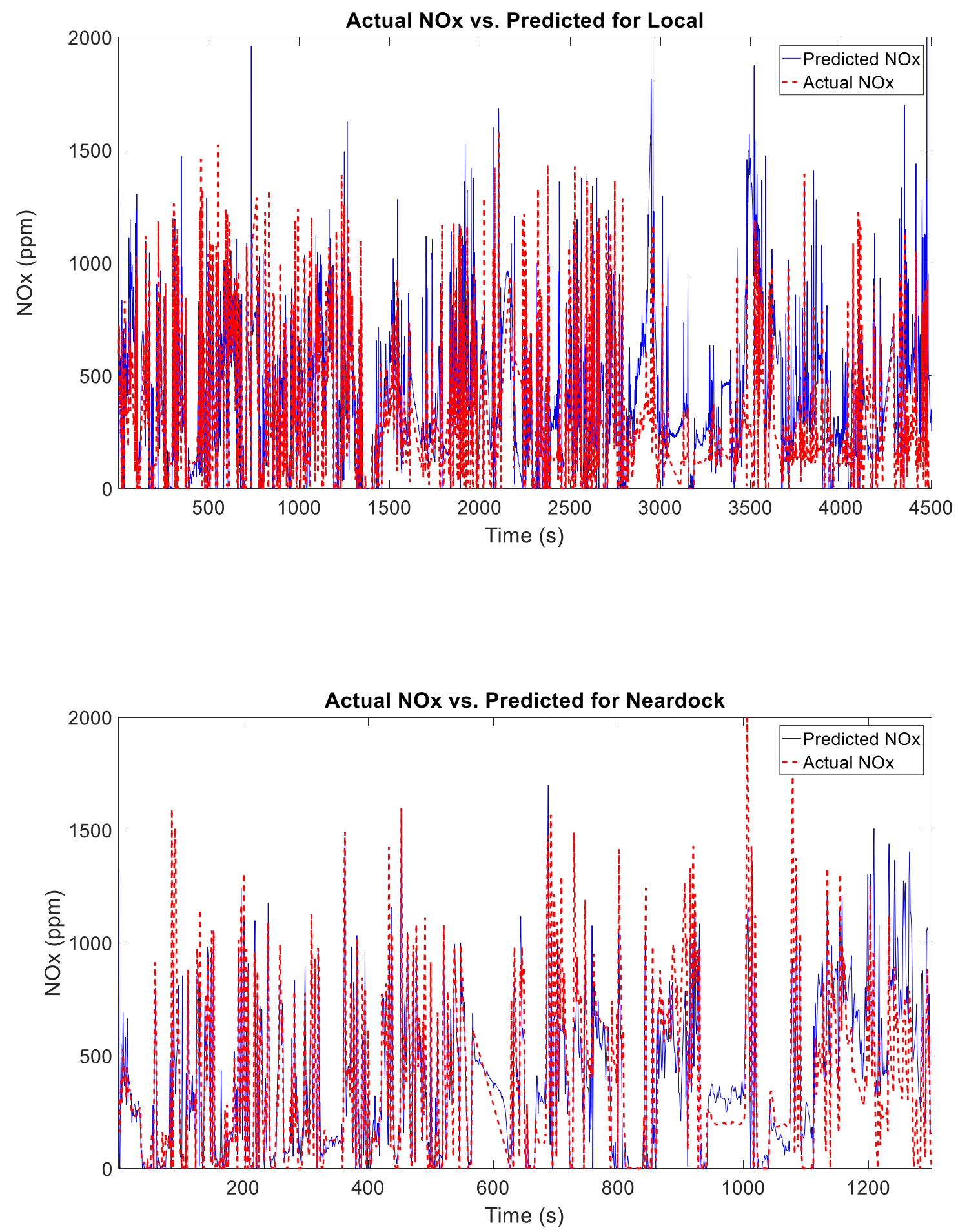

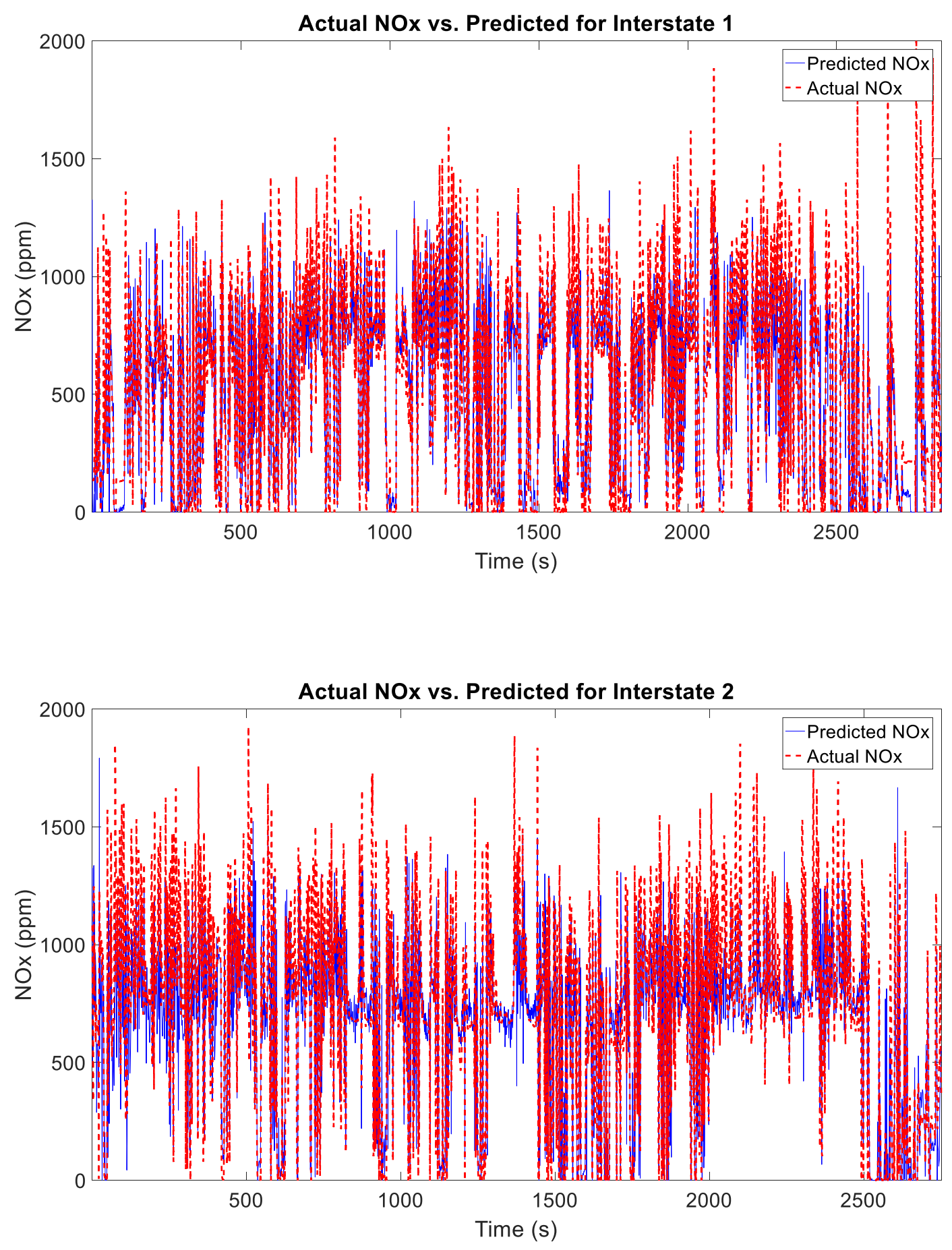

\subsection{APPENDIXI}

Appendix I includes the plots of the predicted versus actual NOx values with data points eliminated when the power was less than twenty percent of the max value. 

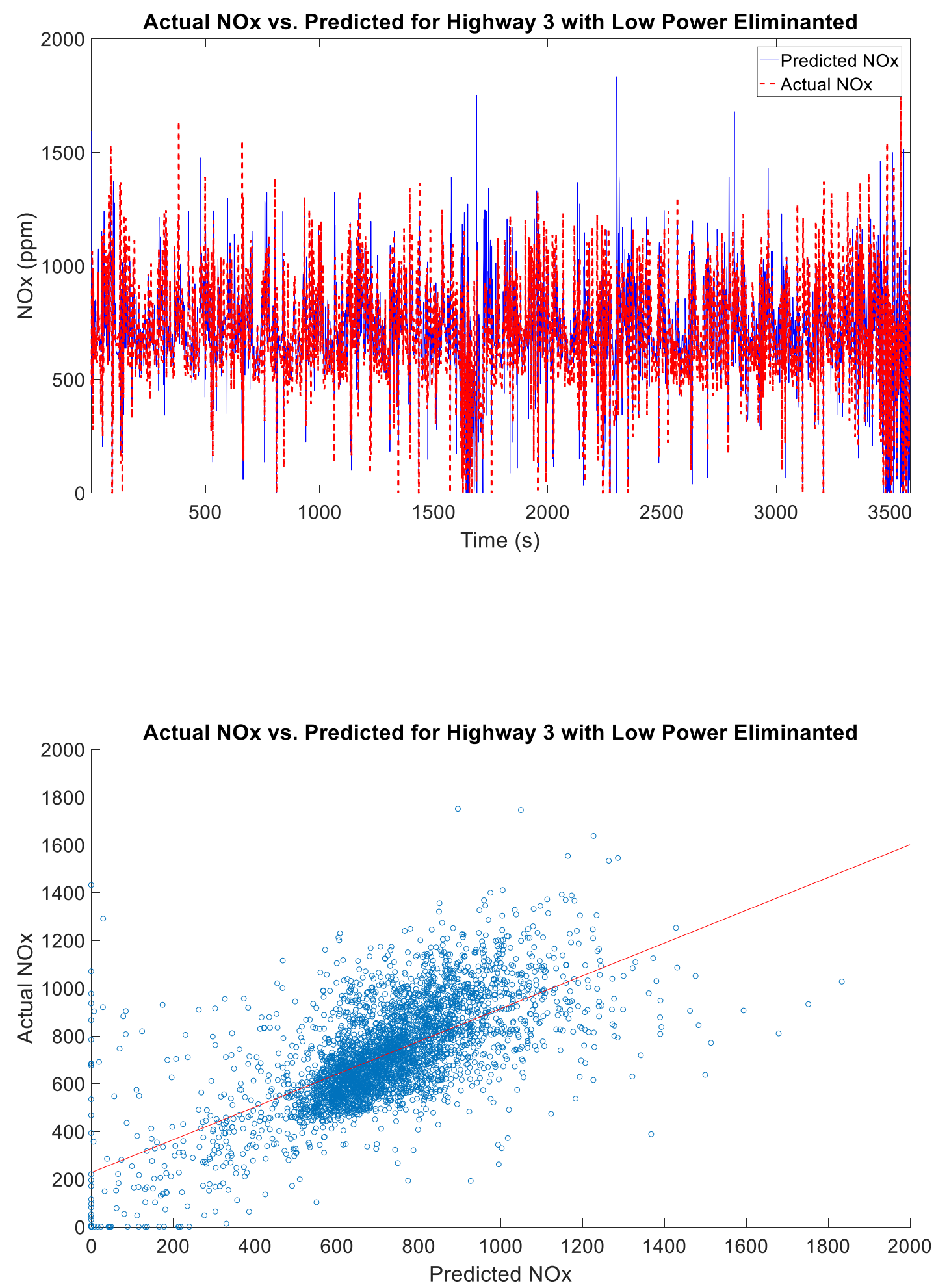

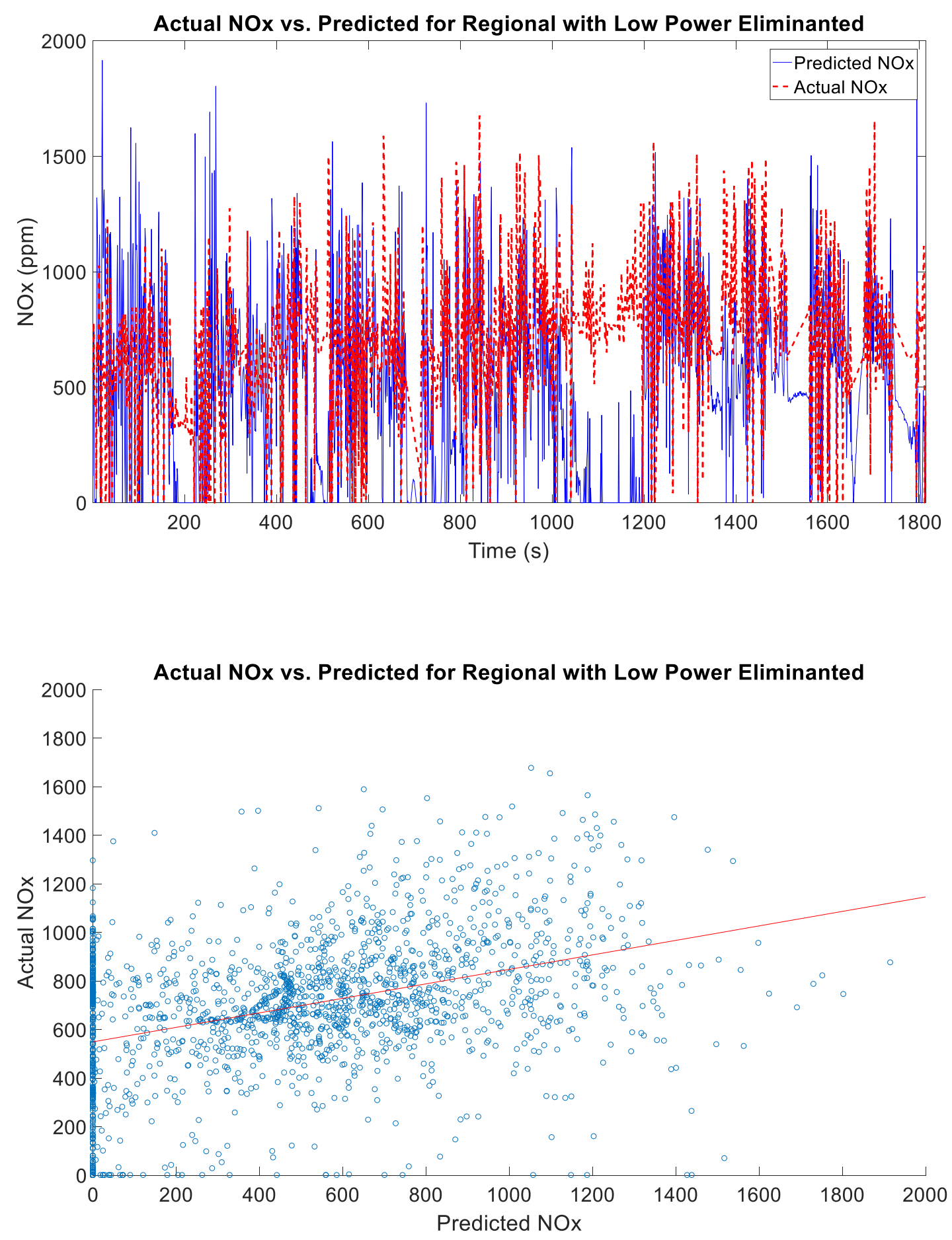

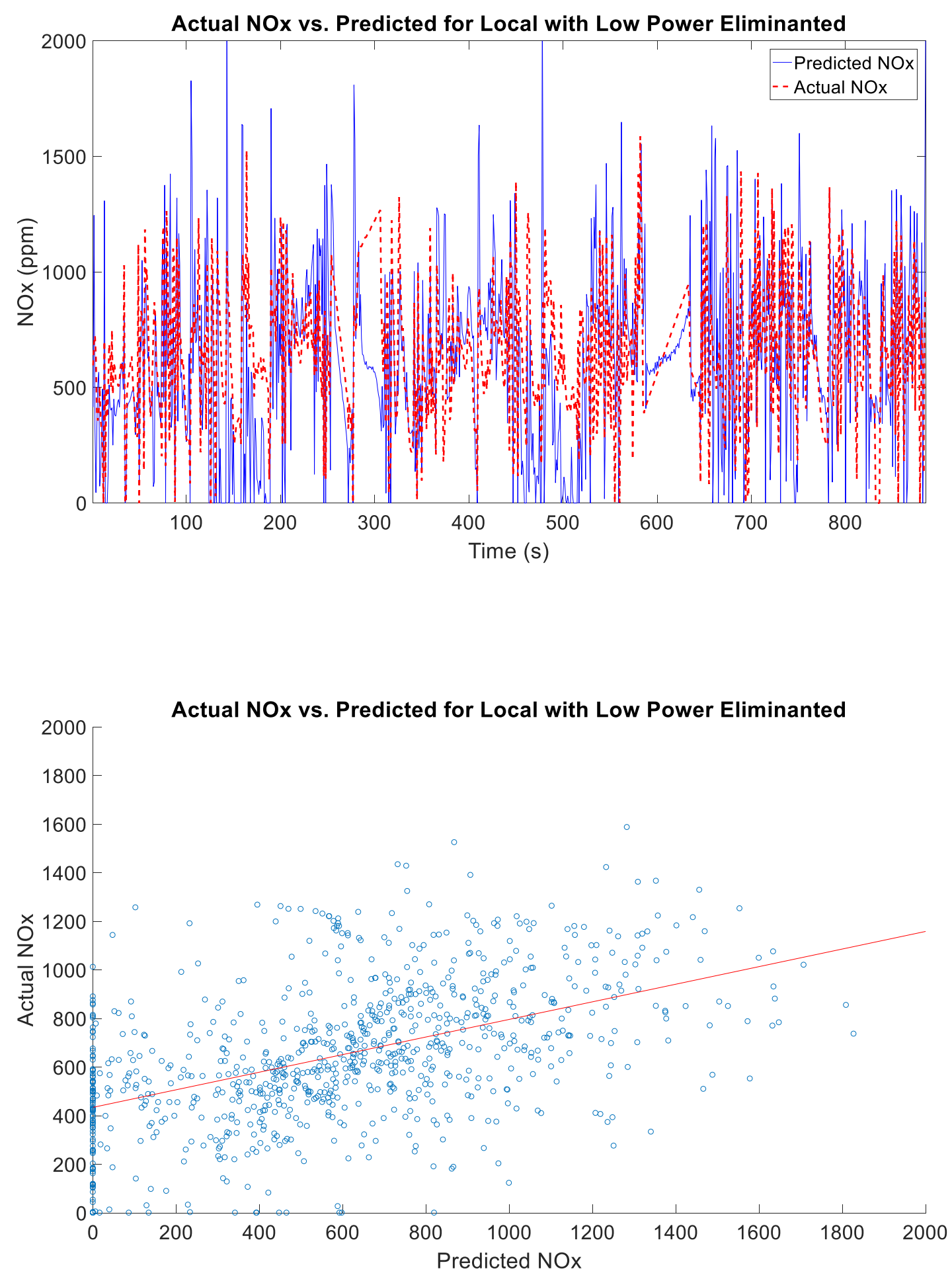

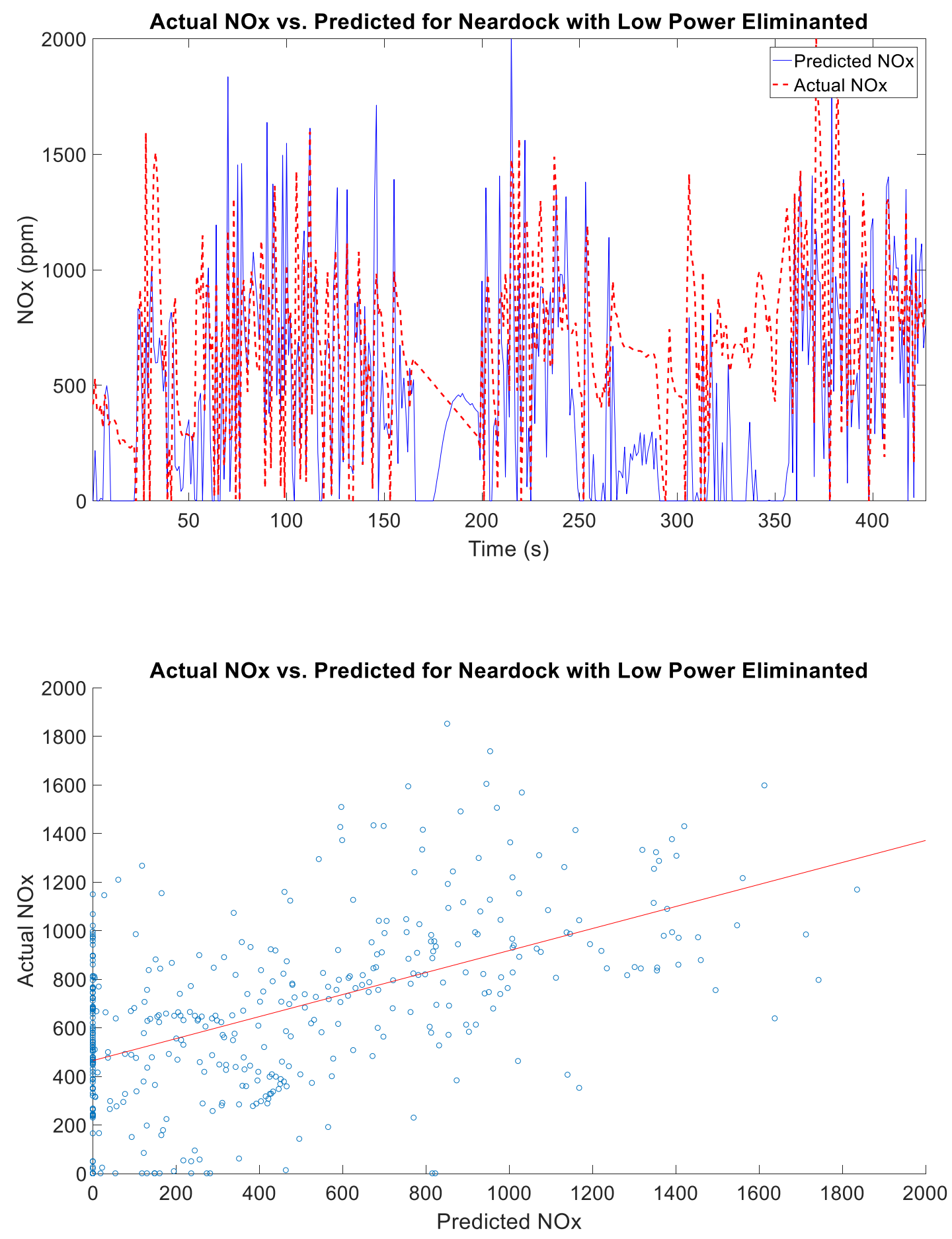

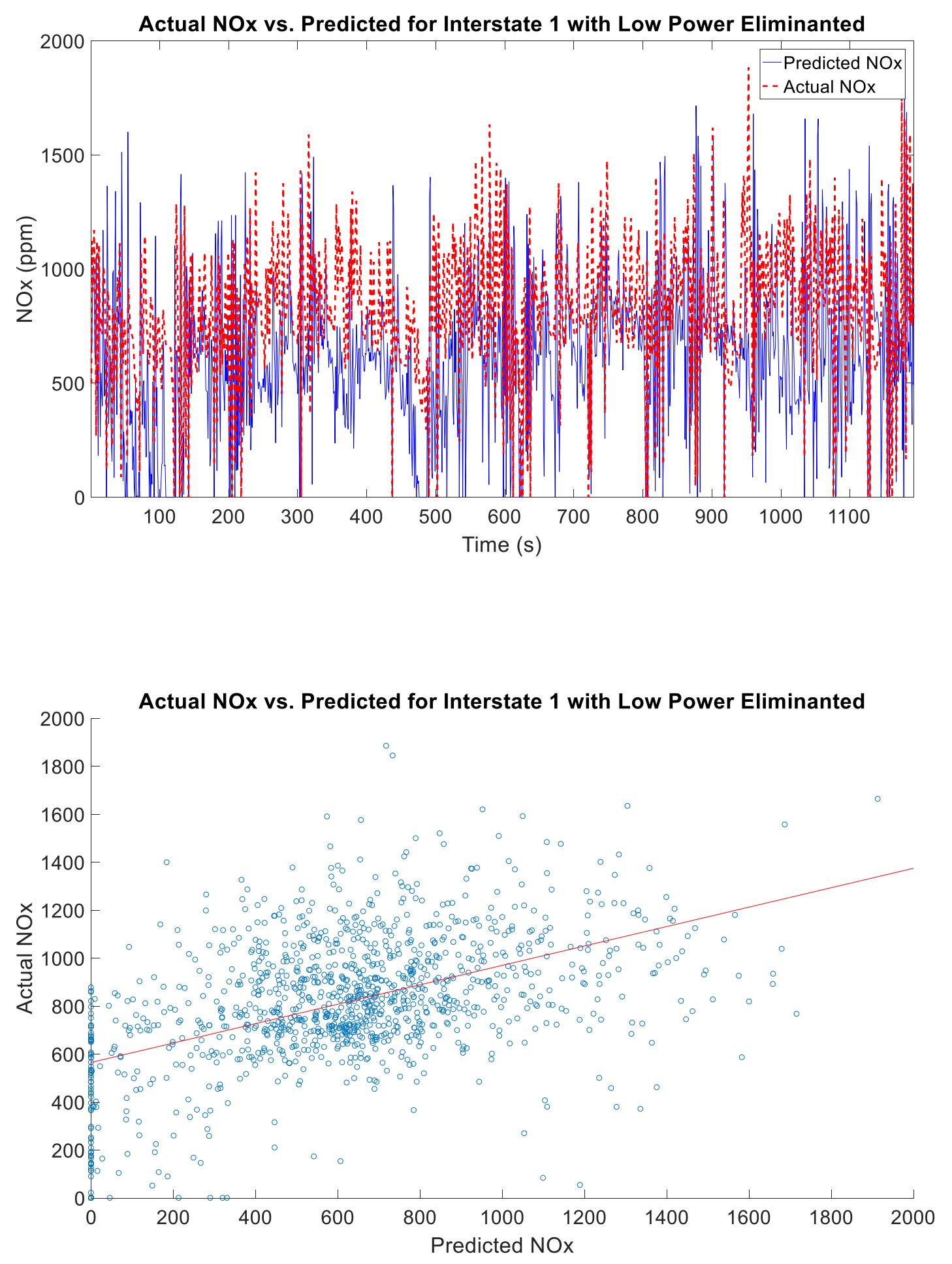

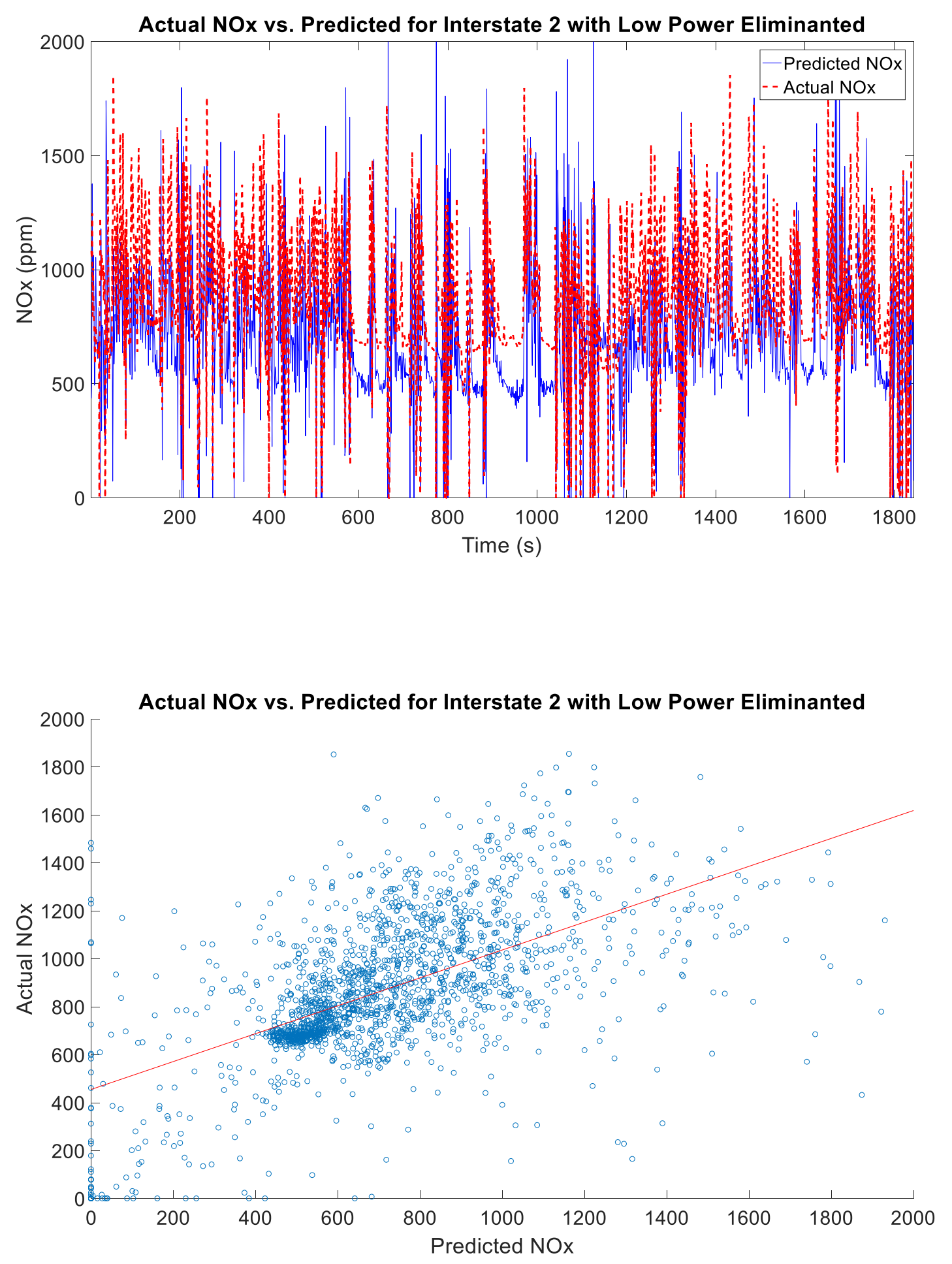

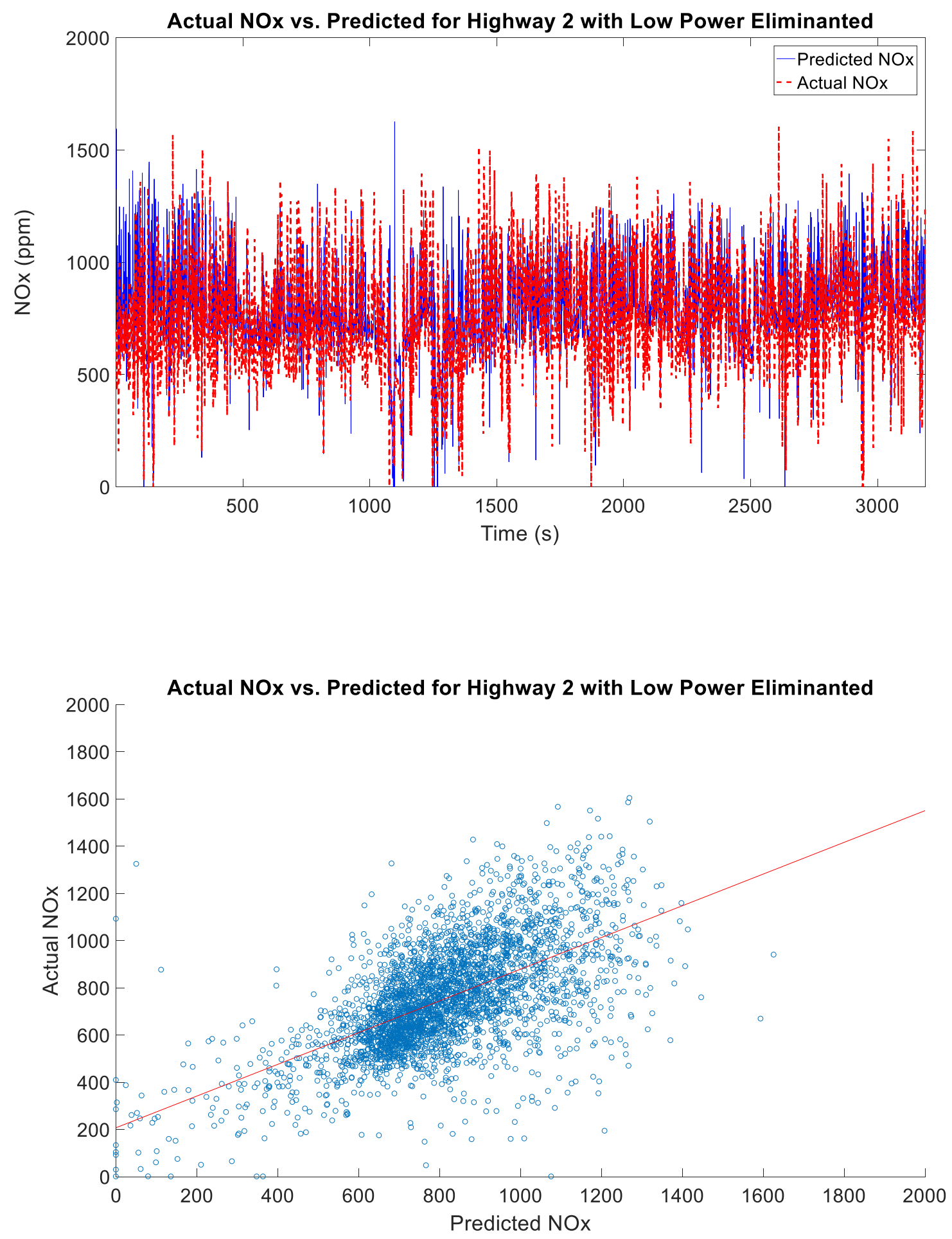

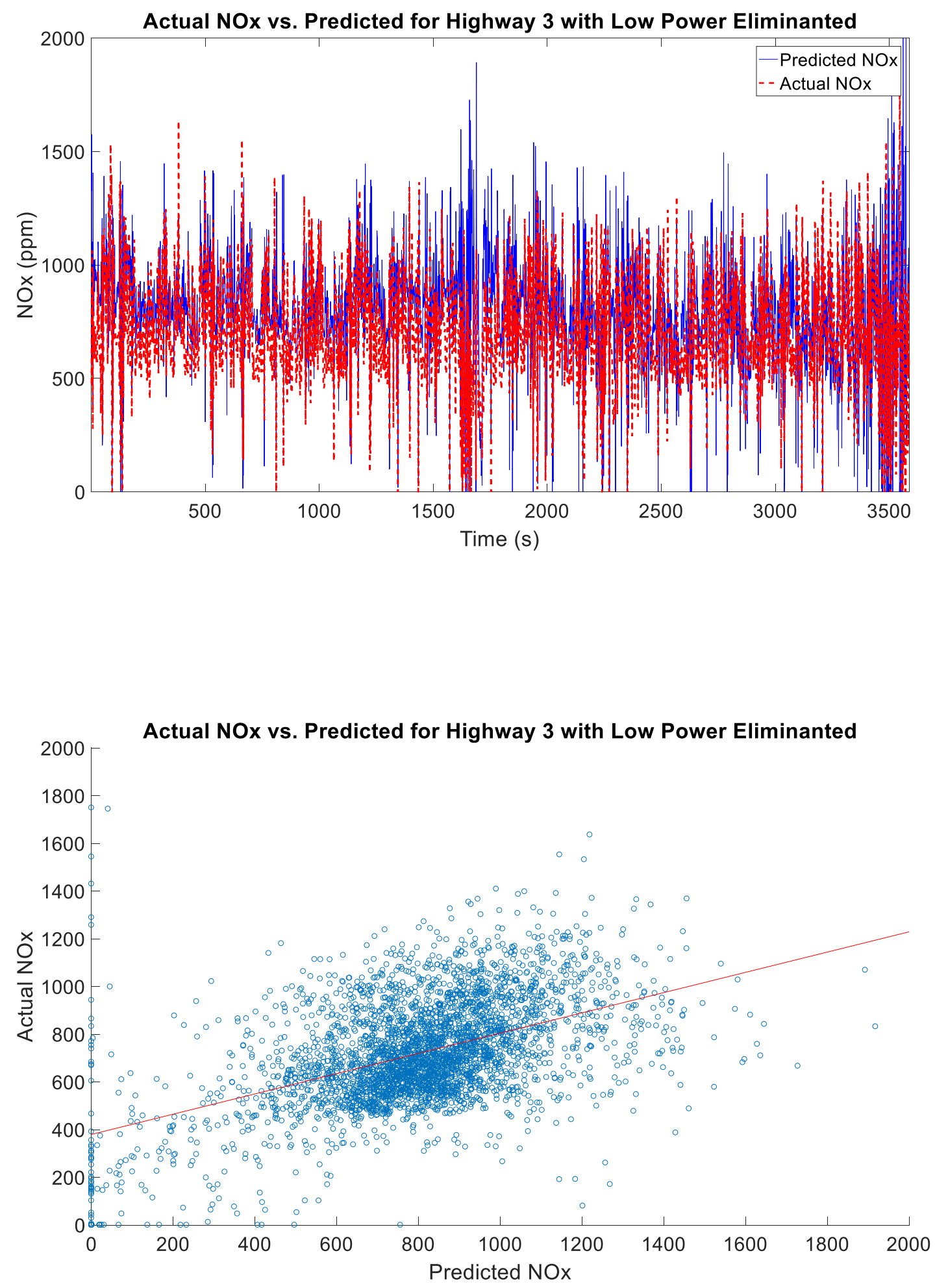

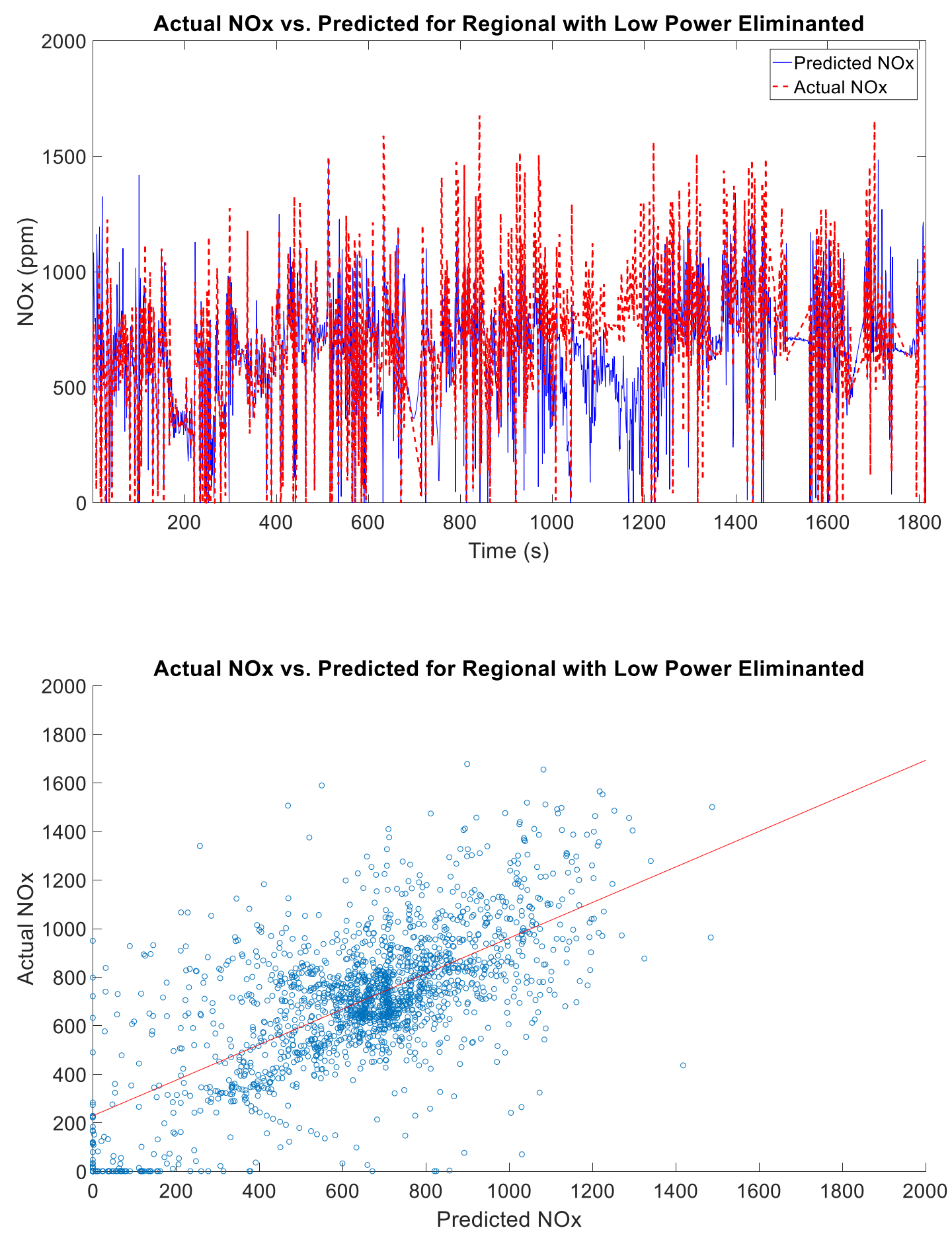

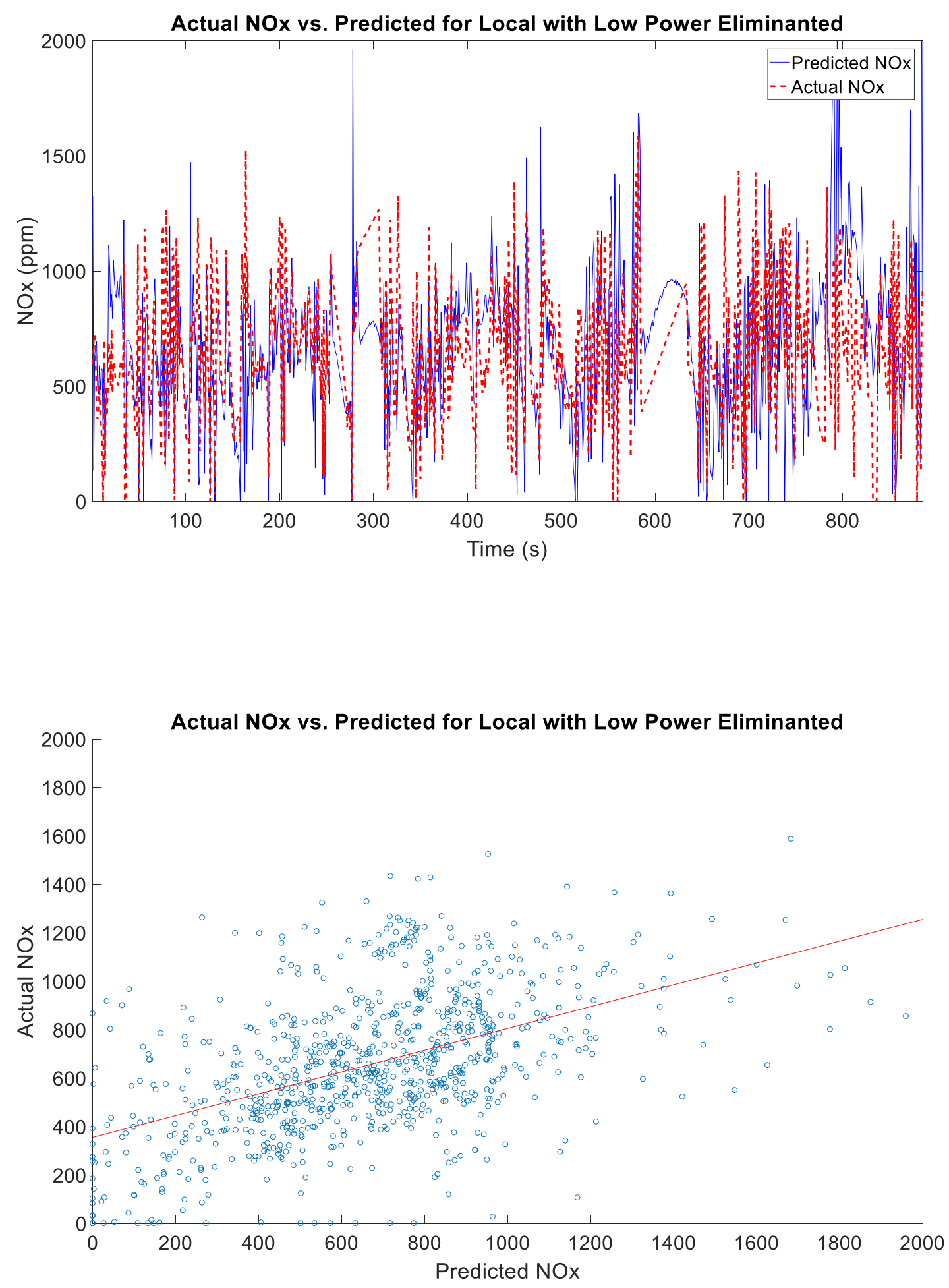

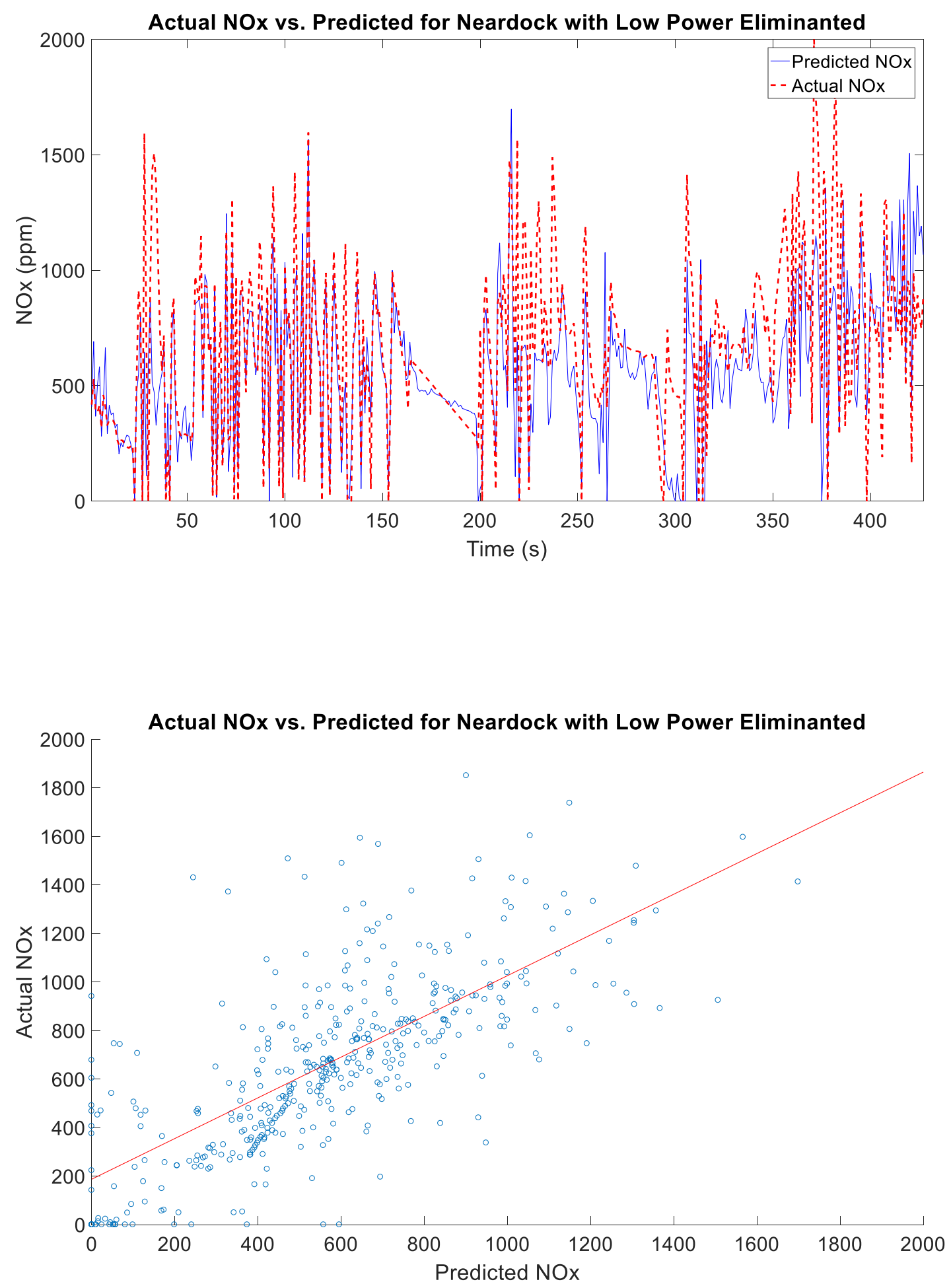

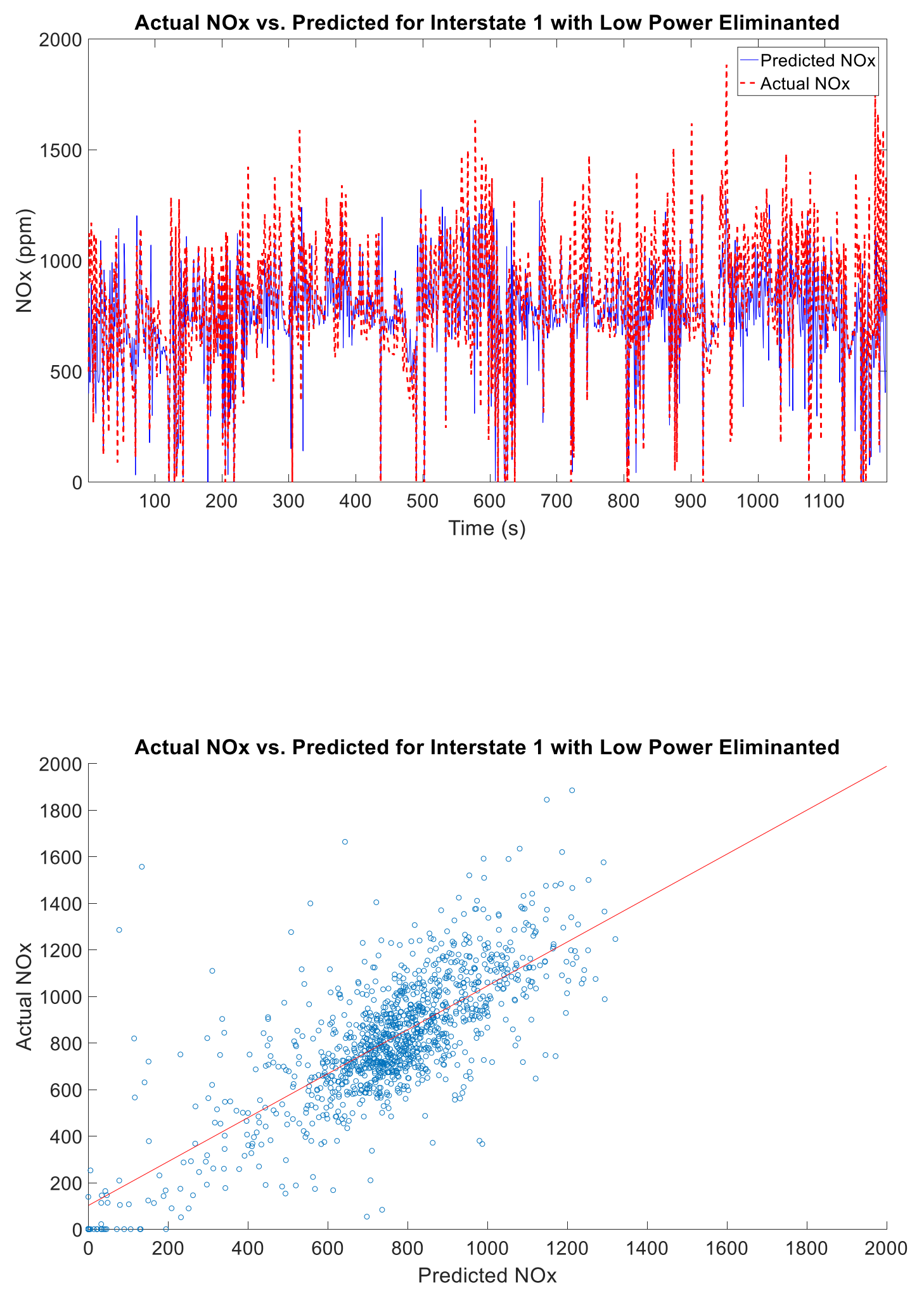

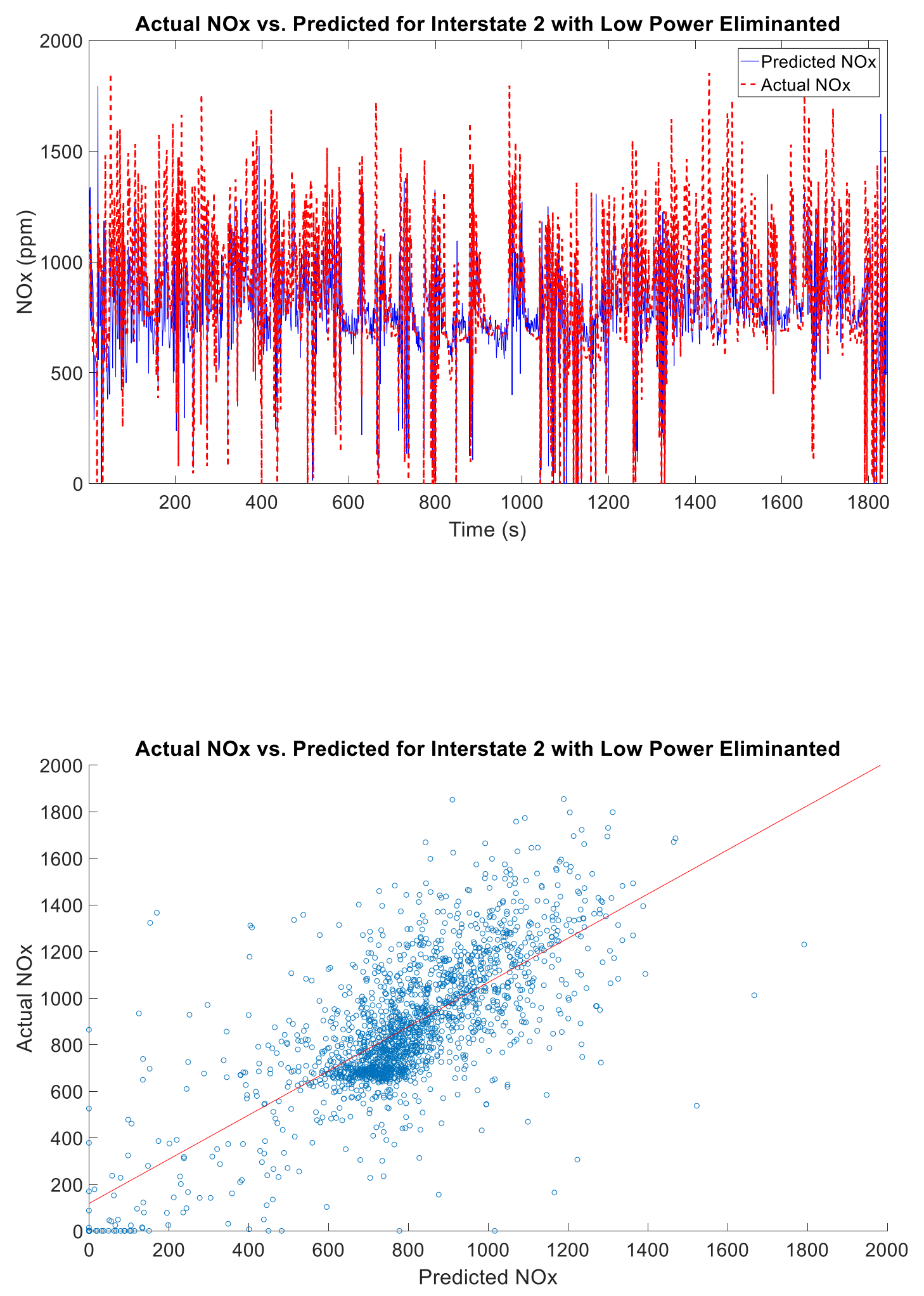